\title{
Iridium-Catalyzed Asymmetric Hydroalkenylation of Norbornene Derivatives
}

\author{
Xin Sun, Xiao-Yan Bai*,b, An-Zhen Li and Bi-Jie Li*,a \\ ${ }^{a}$ Center of Basic Molecular Science (CBMS), Department of Chemistry, Tsinghua University \\ ${ }^{\mathrm{b}}$ College of Chemistry and Material Science, Sichuan Normal University
}

Table of Contents

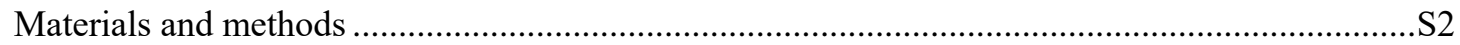

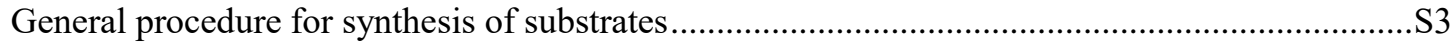

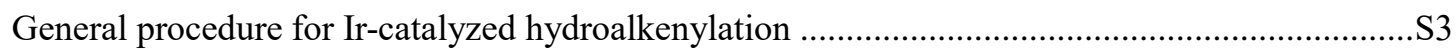

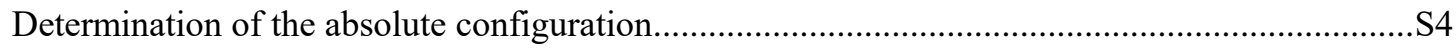

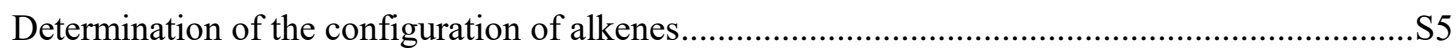

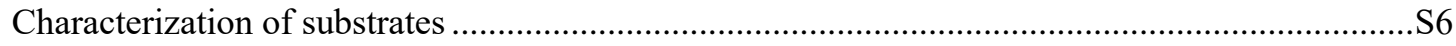

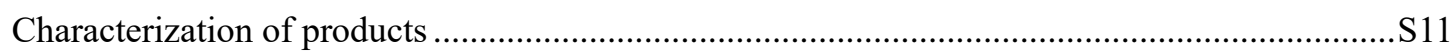

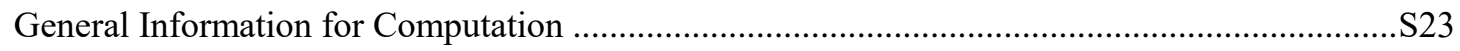

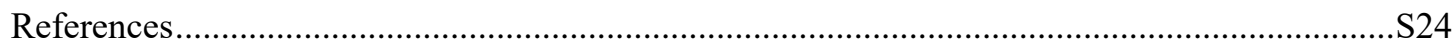

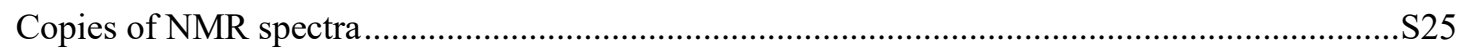

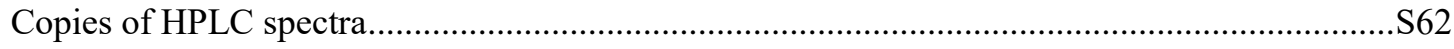




\section{Materials and methods}

Unless otherwise noted, all reactions were assembled on a Schlenk vacuum line or in a glovebox using oven-dried glassware and were stirred with Teflon-coated magnetic stirring bars. All the ligands were purchased from Strem Chemicals and were used as received. $\left[\operatorname{Ir}(\operatorname{cod})_{2}\right] \mathrm{OTf}^{1}$ was prepared according to literature methods. Norbornene, norbornadiene, benzonorbornadiene, 1,4epoxy-1,4-Dihydronaphthalene, acrylic acid, N, N-diethylacrylamide, N-n-butyl acrylamide, nbutyl acrylate, benzyl acrylate, 1-(3-Dimethylaminopropyl)-3-ethylcarbodiimide Hydrochloride (EDCI) and 4-dimethylaminopyridine (DMAP), n-butyllithium and all other reagents for the synthesis of the substrates were purchased from Alfa Aesar, Energy Chemical, Innochem, Bidepharm, JK chemical and were used as received. Anhydrous 1,2-dichloroethane (DCE) was purchase from Acros, which was stored over molecular sieves and used as received. Toluene was degassed by purging with nitrogen and then dried with a solvent purification system containing activated alumina. All work-up and purification procedures were carried out with reagent grade solvents in air. Reaction temperatures above $23{ }^{\circ} \mathrm{C}$ refer to temperatures of an aluminum heating block or a silicon oil bath, which were controlled by an electronic temperature modulator from IKA. NMR spectra were acquired on NMR spectrometer with $400 \mathrm{MHz}$ for ${ }^{1} \mathrm{H}$ NMR and $101 \mathrm{MHz}$ for ${ }^{13} \mathrm{C}$ NMR at the NMR facility at Center of Basic Molecular Science (CBMS). Chemical shifts $(\delta)$ are reported in ppm relative to the residual solvent signal $\left(\delta=7.26\right.$ for ${ }^{1} \mathrm{H}$ NMR and $\delta=77.0$ for ${ }^{13} \mathrm{C}$ NMR). Data for ${ }^{1} \mathrm{H}$ NMR spectra are reported as follows: chemical shift (multiplicity, coupling constants, number of hydrogens). Abbreviations are as follows: s (singlet), d (doublet), $t$ (triplet), $q$ (quartet), m (multiplet), br (broad). Infrared (IR) spectra were recorded on a Bruker FT-IR alpha (ATR mode) spectrophotometer. GC data were obtained on a Shimazu 2010 Plus GC system containing an Rxi®-5ms capillary column. High-resolution mass spectral data was performed on a Thermo Scientific Q Exactive (positive mode) at the Mass Spectrometry Facility, CBMS. Enantiomer excess (ee) values were determined by analytical liquid chromatography (HPLC) analysis on a Shimadzu chromatograph (Daicel chiral columns Chiralpak OD-H, OJ-H, ID (4.6 x $250 \mathrm{~mm})$ ). Specific rotations were measured on a Jasco P-2000 Polarimeter. 


\section{General procedure for synthesis of substrates ${ }^{2,3}$ :}

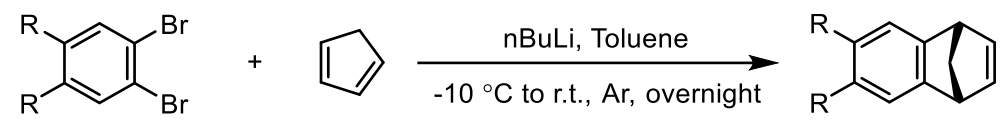

Scheme S1. Synthesis method of NBE derivatives

Substituted 1,2-dibromobenzene (10.0 mmol) and freshly distilled cyclopentadiene (20.0 mmol) were dissolved in toluene $(20 \mathrm{~mL})$ at $0{ }^{\circ} \mathrm{C}$ under Ar atmosphere. $n$-BuLi $(30.0 \mathrm{mmol})$ was added to the solution dropwise over $10 \mathrm{~min}$. After stirring for an additional $10 \mathrm{~min}$ at $0{ }^{\circ} \mathrm{C}$, the resulting suspension was warmed to $r$. t. slowly and stirred overnight. After quenching with $30 \mathrm{~mL} \mathrm{H}_{2} \mathrm{O}$, the reaction mixture was extracted with hexane $(3 \times 50 \mathrm{~mL})$. The combined organic layer was dried over $\mathrm{Na}_{2} \mathrm{SO}_{4}$, filtered and evaporated under reduced pressure to remove the solvent. The residue was purified by column chromatography on silica gel eluting with hexane to provide the product.

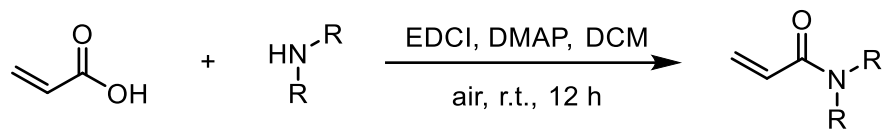

Scheme S2. Synthesis method of $\alpha, \beta$-unsaturated amides

In a $100 \mathrm{~mL}$ round-bottomed flask, acrylic acid $(10.0 \mathrm{mmol})$, EDCI $(2.88 \mathrm{~g}, 15.0 \mathrm{mmol})$, and DMAP (122 mg, $1.00 \mathrm{mmol}$ ) were added. A stir bar was added, and the solid was dissolved in $\mathrm{CH}_{2} \mathrm{Cl}_{2}(20 \mathrm{~mL})$. The resulting solution was treated with amine $(12.0 \mathrm{mmol})$, and stirred at room temperature for $12 \mathrm{~h}$. The reaction mixture was diluted with water $(30 \mathrm{~mL})$ and extracted with DCM (20 $\mathrm{mL} \times 2$ ). The combined organic layer was dried over anhydrous $\mathrm{Na}_{2} \mathrm{SO}_{4}$. After evaporation of the solvent under reduced pressure, the residue was purified by column chromatography on silica gel with EtOAc/hexanes mixture as eluent.

\section{General procedure for Ir-catalyzed hydroalkenylation:}

In an Ar-filled glovebox, $2.8 \mathrm{mg}$ of $\left[\operatorname{Ir}(\mathrm{COD})_{2}\right] \mathrm{OTf}(0.0050 \mathrm{mmol})$ and $4.1 \mathrm{mg}$ of ligand $\mathbf{L 8}$ (0.0060 mmol) were weighed into a one-dram screw-capped vial. $1.0 \mathrm{~mL}$ of DCE was added and the resulting solution was treated with NBE derivative $(0.20 \mathrm{mmol}$ or $0.50 \mathrm{mmol})$ and acrylamide $(0.10 \mathrm{mmol})$. The vial was capped with a Teflon-lined screw cap, removed from the glovebox, and placed in a pre-heated aluminum block at $80^{\circ} \mathrm{C}$ for $14 \mathrm{~h}$. The reaction mixture was directly purified by column chromatography on silica gel with EtOAc/hexanes mixture as eluent.

All of the racemic products were obtained by using racemic BINAP as the ligand under the general procedure for Ir-catalyzed hydroalkenylation described above. 


\section{Determination of the absolute configuration ${ }^{4}$ :}

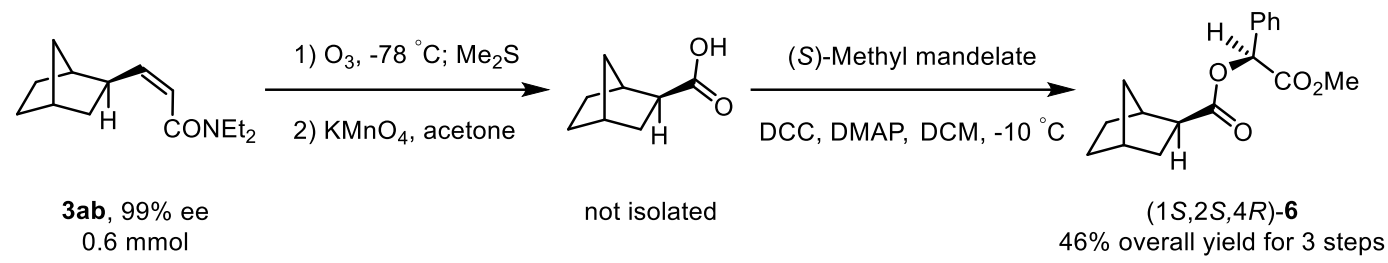

Scheme S3. Derivation of product 3ab

The absolute configuration of the product was determined by conversion to known mandelic acid derivatives according to the established procedure by Parker and RajanBabu. Ozonolysis of 3ab followed by oxidation of the resulting aldehyde gave norbonane-2-carboxylic acid, which was converted into ester of (S)-methyl mandelate by the standard procedure using DCC. The C2hydrogen of the product 6 appears at $\delta 2.86$ (reported 2.87 and 2.88). The benzylic hydrogens of the product 6 appears at $\delta 6.065$ (reported 6.061 and 6.082). Accordingly, the hydroalkenylation product

3ab is assigned to $(1 S, 2 S, 4 R)$.

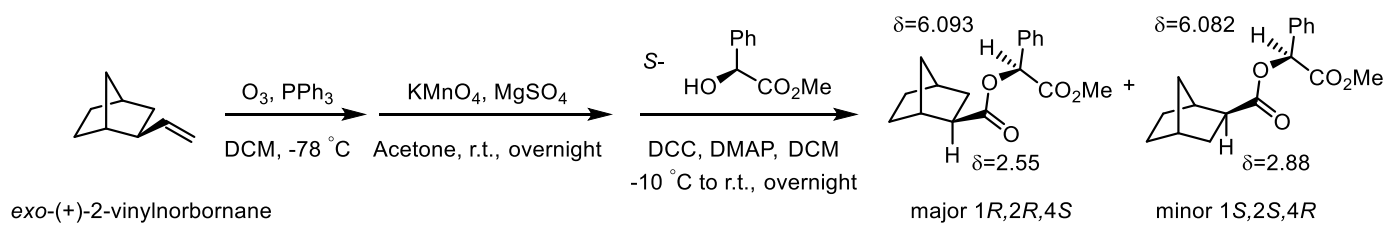

Scheme S4. Absolute Configuration of exo-(+)-2-vinylnorbornane and Configuration of the $(S)$-Mandelate Esters

Ref.: Org. Lett. 2003, 5, 4345-4348

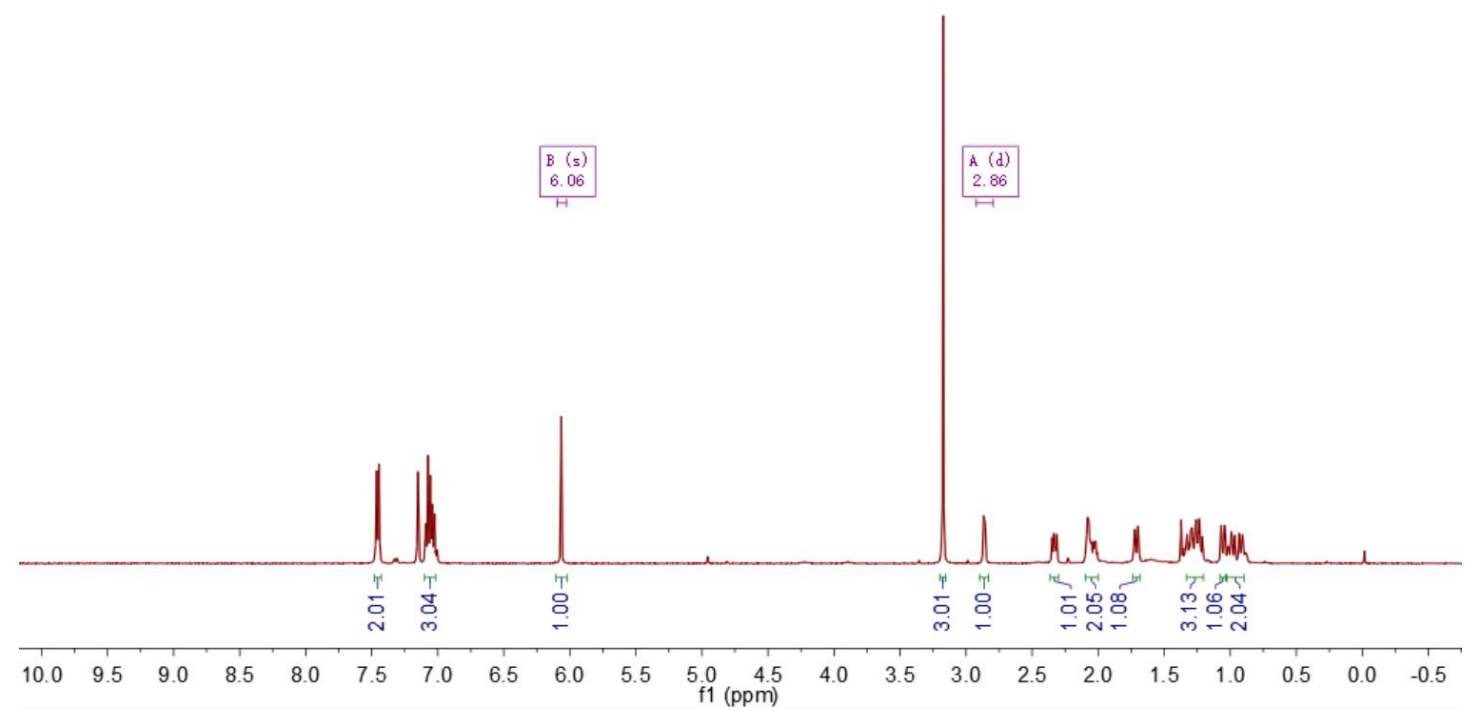

Figure S1. ${ }^{1} \mathbf{H}$ NMR $\left(400 \mathrm{MHz}, \mathrm{C}_{6} \mathrm{D}_{6}\right)$ of $\mathbf{6}$ 
Determination of the configuration of alkenes:

5ab:

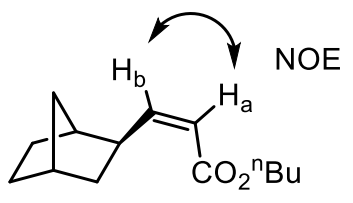

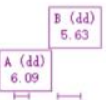

\begin{tabular}{ccc}
$C_{4.13}^{(t)}$ & $\begin{array}{c}\text { (td) } \\
3.31\end{array}$ \\
\hline
\end{tabular}
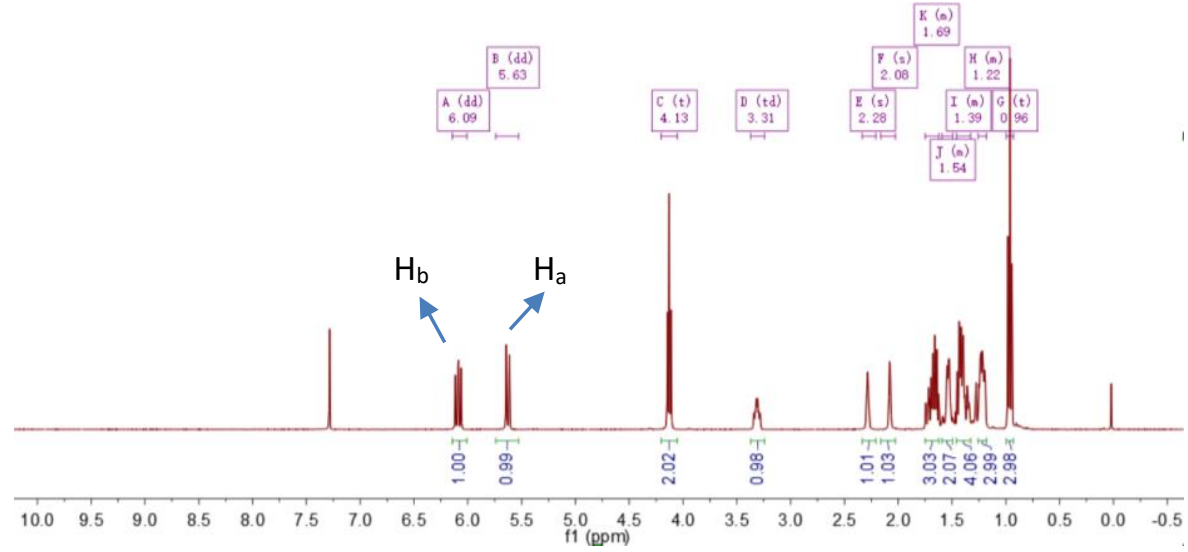

Figure S2. ${ }^{1} \mathbf{H}$ NMR $\left(400 \mathrm{MHz}, \mathrm{CDCl}_{3}\right)$ of $\mathbf{5 a b}$

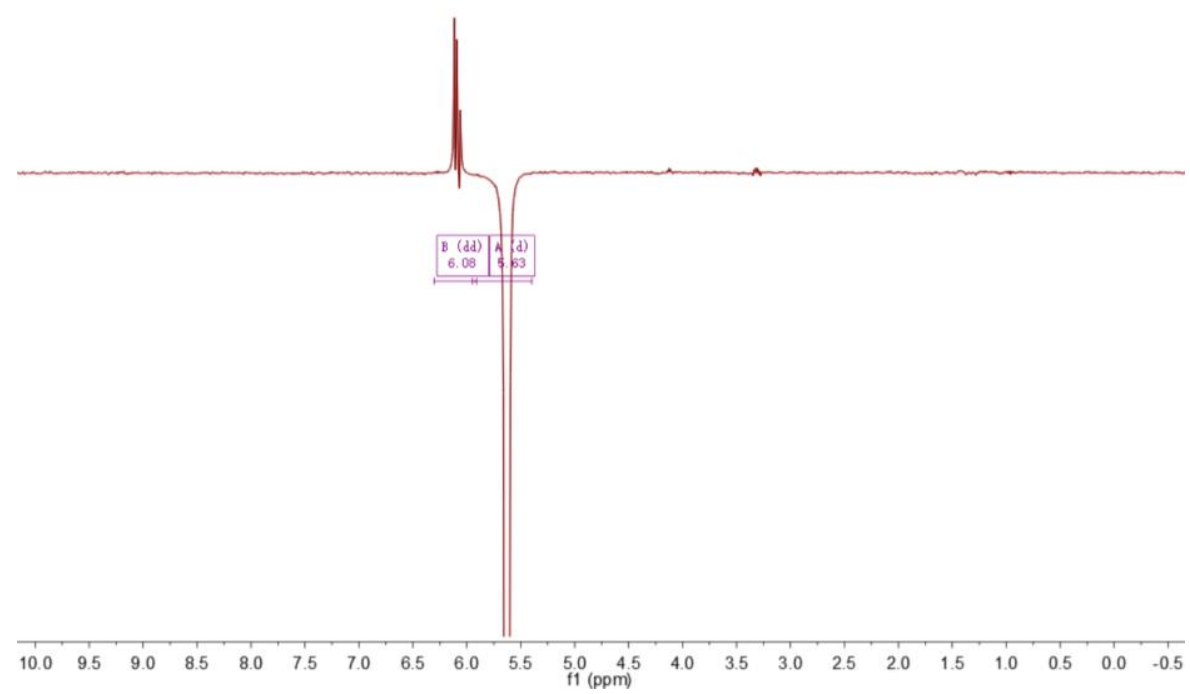

Figure S3. NOE experiments of 5ab

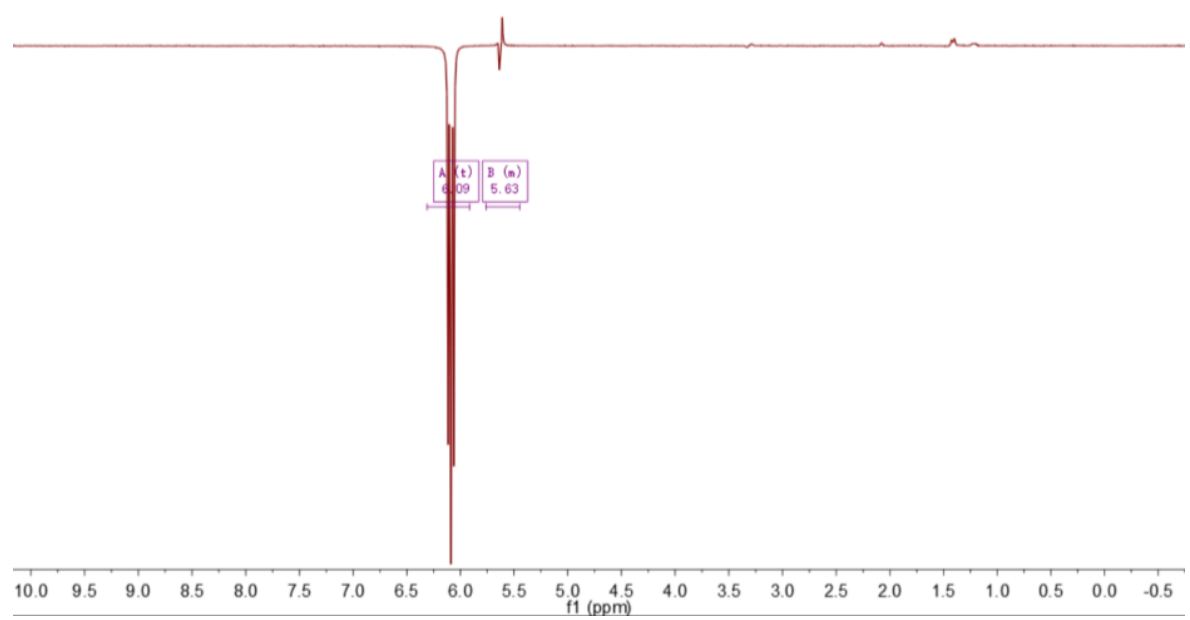

Figure S4. NOE experiments of 5ab 


\section{Characterization of substrates:}

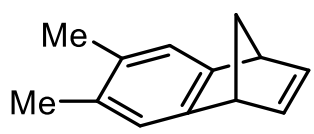

2d: Following the general procedure, $\mathbf{2 d}$ was obtained and purification by silica gel chromatography in $398.8 \mathrm{mg}$ (23\% yield) as a colorless oil. ${ }^{1} \mathbf{H}$ NMR $\left(400 \mathrm{MHz}, \mathrm{CDCl}_{3}\right) \delta 7.09(\mathrm{~s}, 2 \mathrm{H}), 6.82(\mathrm{~s}, 2 \mathrm{H})$, $3.88(\mathrm{~s}, 2 \mathrm{H}), 2.35(\mathrm{~d}, \mathrm{~J}=6.9 \mathrm{~Hz}, 1 \mathrm{H}), 2.25(\mathrm{~s}, 7 \mathrm{H}) .{ }^{13} \mathbf{C}$ NMR $\left(101 \mathrm{MHz}, \mathrm{CDCl}_{3}\right) \delta 149.3,143.1$, 131.7, 123.4, 69.9, 50.0, 19.7. ESI-HR calcd for $\mathrm{C}_{13} \mathrm{H}_{15}{ }^{+}\left([\mathrm{M}+\mathrm{H}]^{+}\right)$171.1168, found 171.1163. IR $v\left(\mathrm{~cm}^{-1}\right) 3004,2976,2933,2863,1463,750$.

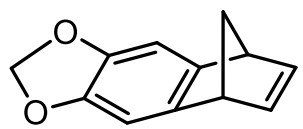

2e: Following the general procedure, $\mathbf{2 e}$ was obtained and purification by silica gel chromatography in $749.6 \mathrm{mg}$ (40\% yield) as a light yellow solid. ${ }^{1} \mathbf{H}$ NMR $\left(400 \mathrm{MHz}, \mathrm{CDCl}_{3}\right) \delta 6.80(\mathrm{~m}, 4 \mathrm{H}), 5.87$ $(\mathrm{d}, \mathrm{J}=23.7 \mathrm{~Hz}, 2 \mathrm{H}), 3.81(\mathrm{~s}, 2 \mathrm{H}), 2.25(\mathrm{dd}, \mathrm{J}=29.2,6.8 \mathrm{~Hz}, 2 \mathrm{H}) .{ }^{13} \mathbf{C ~ N M R}\left(101 \mathrm{MHz}, \mathrm{CDCl}_{3}\right) \delta$ 145.5, 143.7, 143.2, 104.7, 100.7, 70.7, 50.4. ESI-HR calcd for $\mathrm{C}_{12} \mathrm{H}_{11} \mathrm{O}_{2}{ }^{+}\left([\mathrm{M}+\mathrm{H}]^{+}\right)$187.0754, found 187.0749. IR $v\left(\mathrm{~cm}^{-1}\right) 3005,2986,2934,1463,750$. M. P. $74{ }^{\circ} \mathrm{C}$.

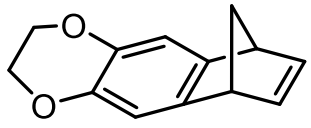

2f: Following the general procedure, $\mathbf{2 f}$ was obtained and purification by silica gel chromatography in $1220.8 \mathrm{mg}$ (61\% yield) as a light yellow solid. ${ }^{1} \mathbf{H}$ NMR $\left(400 \mathrm{MHz}, \mathrm{CDCl}_{3}\right) \delta 6.78(\mathrm{~s}, 2 \mathrm{H}), 6.75$ (t, J = 1.8 Hz, 2H), $4.19(\mathrm{~s}, 4 \mathrm{H}), 3.79(\mathrm{dd}, \mathrm{J}=3.4,1.7 \mathrm{~Hz}, 2 \mathrm{H}), 2.24(\mathrm{ddd}, \mathrm{J}=37.4,19.5,4.3 \mathrm{~Hz}$, 2H). ${ }^{13} \mathbf{C}$ NMR $\left(101 \mathrm{MHz}, \mathrm{CDCl}_{3}\right) \delta 144.2,142.8,139.3,111.8,69.3,64.3,49.8$. ESI-HR calcd for $\mathrm{C}_{13} \mathrm{H}_{13} \mathrm{O}_{2}{ }^{+}\left([\mathrm{M}+\mathrm{H}]^{+}\right)$201.0910, found 201.0905. IR $v\left(\mathrm{~cm}^{-1}\right) 3007,2989,2967,2869,1462,750 . \mathbf{M}$. P. $91{ }^{\circ} \mathrm{C}$.

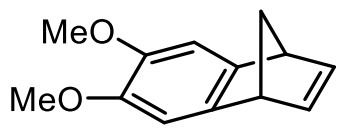


2g: Following the general procedure, $\mathbf{2 g}$ was obtained and purification by silica gel chromatography in $208.3 \mathrm{mg}$ (10\% yield) as a yellow solid. ${ }^{1} \mathbf{H}$ NMR $\left(400 \mathrm{MHz}, \mathrm{CDCl}_{3}\right) \delta 6.92(\mathrm{~s}, 2 \mathrm{H}), 6.80(\mathrm{~s}, 2 \mathrm{H})$, $3.85(\mathrm{~m}, 8 \mathrm{H}), 2.25(\mathrm{dd}, \mathrm{J}=33.2,6.8 \mathrm{~Hz}, 2 \mathrm{H}) .{ }^{13} \mathbf{C} \mathbf{N M R}\left(101 \mathrm{MHz}, \mathrm{CDCl}_{3}\right) \delta 145.4,144.0,143.2$, 107.7, 70.7, 56.4, 50.5. ESI-HR calcd for $\mathrm{C}_{13} \mathrm{H}_{15} \mathrm{O}_{2}{ }^{+}\left([\mathrm{M}+\mathrm{H}]^{+}\right)$203.1067, found 203.1063. IR v $\left(\mathrm{cm}^{-1}\right) 3019,2990,1479,749$. M. P. $78^{\circ} \mathrm{C}$.

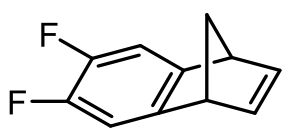

$\mathbf{2 h}$ : Following the general procedure, $\mathbf{2 h}$ was obtained and purification by silica gel chromatography in $879.5 \mathrm{mg}$ (49\% yield) as a colorless oil. ${ }^{1} \mathbf{H}$ NMR $\left(400 \mathrm{MHz}, \mathrm{CDCl}_{3}\right) \delta 7.05(\mathrm{t}, \mathrm{J}=8.3 \mathrm{~Hz}, 2 \mathrm{H})$, $6.81(\mathrm{t}, \mathrm{J}=1.8 \mathrm{~Hz}, 2 \mathrm{H}), 3.93-3.84(\mathrm{~m}, 2 \mathrm{H}), 2.30(\mathrm{dd}, \mathrm{J}=32.5,7.2 \mathrm{~Hz}, 2 \mathrm{H}) .{ }^{13} \mathbf{C}$ NMR $(101 \mathrm{MHz}$ $\left.\mathrm{CDCl}_{3}\right) \delta$ 147.7-147.5 (m, 1C), $146.9(\mathrm{dd}, \mathrm{J}=245.1,14.8 \mathrm{~Hz}, 1 \mathrm{C}), 142.9(\mathrm{~s}, 1 \mathrm{C}), 111.3$ (dd, J = 12.8, $7.1 \mathrm{~Hz}, 1 \mathrm{C}), 70.3(\mathrm{~s}, 1 \mathrm{C}), 50.2(\mathrm{~s}, 1 \mathrm{C})$. ESI-HR calcd for $\mathrm{C}_{11} \mathrm{H}_{9} \mathrm{~F}_{2}{ }^{+}\left([\mathrm{M}+\mathrm{H}]^{+}\right) 179.0667$, found 179.0673. IR $v\left(\mathrm{~cm}^{-1}\right) 3005,2989,1469,750$.<smiles>C=CC(=O)N(Cc1ccccc1)Cc1ccccc1</smiles>

1b: Following the general procedure, $\mathbf{1 b}$ was obtained and purification by silica gel chromatography in $1022.4 \mathrm{mg}$ (41\% yield) as a colorless oil. ${ }^{1} \mathbf{H}$ NMR $\left(400 \mathrm{MHz}, \mathrm{CDCl}_{3}\right) \delta 7.41-7.27(\mathrm{~m}, 8 \mathrm{H}), 7.18$ $(\mathrm{d}, \mathrm{J}=7.3 \mathrm{~Hz}, 2 \mathrm{H}), 6.63(\mathrm{dd}, \mathrm{J}=16.7,10.2 \mathrm{~Hz}, 1 \mathrm{H}), 6.51(\mathrm{dd}, \mathrm{J}=16.7,2.3 \mathrm{~Hz}, 1 \mathrm{H}), 5.74(\mathrm{dd}, \mathrm{J}=$ 10.1, $2.2 \mathrm{~Hz}, 1 \mathrm{H}), 4.67$ (s, 2H), 4.52 (s, 2H). ${ }^{13} \mathrm{C}$ NMR (101 MHz, $\left.\mathrm{CDCl}_{3}\right) \delta$ 166.8, 137.0, 136.4, 128.8, 128.8, 128.5, 128.2, 127.5, 127.5, 127.3, 126.4, 49.8, 48.4. ESI-HR calcd for $\mathrm{C}_{17} \mathrm{H}_{18} \mathrm{NO}^{+}$ $\left([\mathrm{M}+\mathrm{H}]^{+}\right)$252.1383, found 252.1377. IR $v\left(\mathrm{~cm}^{-1}\right)$ 3086, 3063, 3006, 1649, 1615, 1440, 750.<smiles>C=CC(=O)N(CCOC)CCOC</smiles>

1c: Following the general procedure, $1 \mathbf{c}$ was obtained and purification by silica gel chromatography in $487.1 \mathrm{mg}$ (26\% yield) as a colorless oil. ${ }^{1} \mathbf{H}$ NMR $\left(400 \mathrm{MHz}, \mathrm{CDCl}_{3}\right) \delta 6.63(\mathrm{dd}, \mathrm{J}=16.8,10.4$ 
Hz, 1H), $6.31(\mathrm{dd}, \mathrm{J}=16.8,2.1 \mathrm{~Hz}, 1 \mathrm{H}), 5.64(\mathrm{dd}, \mathrm{J}=10.4,2.1 \mathrm{~Hz}, 1 \mathrm{H}), 3.60(\mathrm{t}, \mathrm{J}=5.6 \mathrm{~Hz}, 4 \mathrm{H})$, $3.54(\mathrm{t}, \mathrm{J}=5.0 \mathrm{~Hz}, 2 \mathrm{H}), 3.48(\mathrm{t}, \mathrm{J}=5.8 \mathrm{~Hz}, 2 \mathrm{H}), 3.31(\mathrm{~s}, 6 \mathrm{H}) .{ }^{13} \mathbf{C} \mathbf{N M R}\left(101 \mathrm{MHz}, \mathrm{CDCl}_{3}\right) \delta$ 166.7, 128.0, 127.6, 71.1, 70.9, 59.0, 58.7, 48.8, 47.0. ESI-HR calcd for $\mathrm{C}_{9} \mathrm{H}_{18} \mathrm{NO}_{3}{ }^{+}\left([\mathrm{M}+\mathrm{H}]^{+}\right)$188.1281, found 188.1278. IR $v\left(\mathrm{~cm}^{-1}\right) 3005,2991,2893,1645,1609,1448,747$.<smiles>C=CC(=O)N1CCCCC1</smiles>

1d: Following the general procedure, 1d was obtained and purification by silica gel chromatography in $679.5 \mathrm{mg}$ (49\% yield) as a colorless oil. ${ }^{1} \mathbf{H}$ NMR $\left(400 \mathrm{MHz}, \mathrm{CDCl}_{3}\right) \delta 6.57-6.39(\mathrm{~m}, 1 \mathrm{H}), 6.19$ $6.06(\mathrm{~m}, 1 \mathrm{H}), 5.59-5.47(\mathrm{~m}, 1 \mathrm{H}), 3.43(\mathrm{~d}, \mathrm{~J}=44.1 \mathrm{~Hz}, 4 \mathrm{H}), 1.63-1.36(\mathrm{~m}, 6 \mathrm{H}) .{ }^{13} \mathbf{C}$ NMR $(101$ $\left.\mathrm{MHz}, \mathrm{CDCl}_{3}\right) \delta 164.9,127.8,126.6,46.6,42.7,26.3,25.2,24.2$. ESI-HR calcd for $\mathrm{C}_{8} \mathrm{H}_{14} \mathrm{NO}^{+}$ $\left([\mathrm{M}+\mathrm{H}]^{+}\right)$140.1070, found 140.1067. IR $v\left(\mathrm{~cm}^{-1}\right) 3004,2935,2855,1644,1608,1439,750$.<smiles>C=CC(=O)N1CCOCC1</smiles>

1e: Following the general procedure, 1e was obtained and purification by silica gel chromatography in $299.6 \mathrm{mg}$ (21\% yield) as a colorless oil. ${ }^{1} \mathbf{H}$ NMR $\left(400 \mathrm{MHz}, \mathrm{CDCl}_{3}\right) \delta 6.51-6.41(\mathrm{~m}, 1 \mathrm{H}), 6.21$ (m, 1H), 5.69-5.57 (m, 1H), 3.54 (m, 8H). ${ }^{13} \mathbf{C}$ NMR (101 MHz, $\left.\mathrm{CDCl}_{3}\right) \delta$ 165.2, 127.9, 126.9, 66.5, 46.0, 42.0. ESI-HR calcd for $\mathrm{C}_{7} \mathrm{H}_{12} \mathrm{NO}_{2}{ }^{+}\left([\mathrm{M}+\mathrm{H}]^{+}\right)$142.0863, found 142.0860. IR $v\left(\mathrm{~cm}^{-1}\right)$ 3004, $2967,2857,1645,1613,1473,750$.<smiles>C=C(C)C(=O)N(CC)CC</smiles>

1f: Following the general procedure, $1 \mathbf{f}$ was obtained and purification by silica gel chromatography in $650.9 \mathrm{mg}$ (46\% yield) as a colorless oil. ${ }^{1} \mathbf{H}$ NMR $\left(400 \mathrm{MHz}, \mathrm{CDCl}_{3}\right) \delta 5.07-5.04(\mathrm{~m}, 1 \mathrm{H}), 4.96$ $(\mathrm{d}, \mathrm{J}=1.0 \mathrm{~Hz}, 1 \mathrm{H}), 3.34(\mathrm{~s}, 4 \mathrm{H}), 1.91(\mathrm{~d}, \mathrm{~J}=1.2 \mathrm{~Hz}, 3 \mathrm{H}), 1.10(\mathrm{t}, \mathrm{J}=7.1 \mathrm{~Hz}, 6 \mathrm{H}) .{ }^{13} \mathbf{C}$ NMR $(101$ MHz, $\left.\mathrm{CDCl}_{3}\right) \delta 172.0,141.3,113.8,42.5,38.5,20.5,14.3,12.7$. ESI-HR calcd for $\mathrm{C}_{8} \mathrm{H}_{16} \mathrm{NO}^{+}$ $\left([\mathrm{M}+\mathrm{H}]^{+}\right)$142.1226, found 142.1225. IR $v\left(\mathrm{~cm}^{-1}\right) 3028,2973,2875,1644,1618,750$. 
$\stackrel{\mathrm{O}}{\mathrm{H}}_{-i \mathrm{Pr}}$

1h: Following the general procedure, $\mathbf{1 h}$ was obtained and purification by silica gel chromatography in $609.9 \mathrm{mg}$ (54\% yield) as a white solid. ${ }^{1} \mathbf{H}$ NMR $\left(400 \mathrm{MHz}, \mathrm{CDCl}_{3}\right) \delta 6.25(\mathrm{dd}, \mathrm{J}=17.0,1.4 \mathrm{~Hz}$, 1H), $6.05(\mathrm{dd}, \mathrm{J}=17.0,10.3 \mathrm{~Hz}, 1 \mathrm{H}), 5.61(\mathrm{dd}, \mathrm{J}=10.3,1.4 \mathrm{~Hz}, 1 \mathrm{H}), 5.46(\mathrm{~s}, 1 \mathrm{H}), 4.22-4.09(\mathrm{~m}$, $1 \mathrm{H}), 1.18(\mathrm{~d}, \mathrm{~J}=6.6 \mathrm{~Hz}, 6 \mathrm{H}) .{ }^{13} \mathbf{C} \mathbf{~ N M R}\left(101 \mathrm{MHz}, \mathrm{CDCl}_{3}\right) \delta 164.6,131.2,126.0,41.4$, 22.7. ESIHR calcd for $\mathrm{C}_{6} \mathrm{H}_{12} \mathrm{NO}^{+}\left([\mathrm{M}+\mathrm{H}]^{+}\right)$114.0913, found 114.0914. IR $v\left(\mathrm{~cm}^{-1}\right) 3271,3088,2974,1656$, 1622, 1543, 751 . M. P. $78^{\circ} \mathrm{C}$.<smiles>C=CC(=O)Nc1ccc(Br)cc1</smiles>

1i: Following the general procedure, $\mathbf{1 i}$ was obtained and purification by silica gel chromatography in $346.5 \mathrm{mg}$ (15\% yield) as a white solid. ${ }^{1} \mathrm{H}$ NMR (400 MHz, $d_{6}$-DMSO) $\delta 10.26(\mathrm{~s}, 1 \mathrm{H}), 7.64(\mathrm{~d}$, $\mathrm{J}=8.8 \mathrm{~Hz}, 2 \mathrm{H}), 7.51(\mathrm{~d}, \mathrm{~J}=8.8 \mathrm{~Hz}, 2 \mathrm{H}), 6.42(\mathrm{dd}, \mathrm{J}=17.0,10.0 \mathrm{~Hz}, 1 \mathrm{H}), 6.27(\mathrm{dd}, \mathrm{J}=17.0,1.8$ $\mathrm{Hz}, 1 \mathrm{H}), 5.78(\mathrm{dd}, \mathrm{J}=10.0,1.9 \mathrm{~Hz}, 1 \mathrm{H}) .{ }^{13} \mathbf{C}$ NMR $\left(101 \mathrm{MHz}, d_{6}\right.$-DMSO) $\delta 163.7,138.8,132.1$, 132.1, 127.7, 121.7, 115.6. ESI-HR calcd for $\mathrm{C}_{9} \mathrm{H}_{9} \mathrm{BrNO}^{+}\left([\mathrm{M}+\mathrm{H}]^{+}\right)$225.9862, found 225.9859. IR $v\left(\mathrm{~cm}^{-1}\right) 3274,2958,2926,1665,1606,1414,1379,751$. M. P. $203{ }^{\circ} \mathrm{C}$.<smiles>C=CC(=O)NCc1ccc(Cl)cc1</smiles>

$\mathbf{1 j}$ : Following the general procedure, $\mathbf{1} \mathbf{j}$ was obtained and purification by silica gel chromatography in $756.0 \mathrm{mg}$ (39\% yield) as a white solid. ${ }^{1} \mathbf{H}$ NMR (400 MHz, $\left.\mathrm{CDCl}_{3}\right) \delta 7.33-7.27$ (m, 2H), 7.22 $(\mathrm{d}, \mathrm{J}=8.6 \mathrm{~Hz}, 2 \mathrm{H}), 6.32(\mathrm{dd}, \mathrm{J}=17.0,1.4 \mathrm{~Hz}, 1 \mathrm{H}), 6.11(\mathrm{dd}, \mathrm{J}=17.0,10.3 \mathrm{~Hz}, 1 \mathrm{H}), 5.98(\mathrm{~s}, 1 \mathrm{H})$, $5.68(\mathrm{dd}, \mathrm{J}=10.3,1.4 \mathrm{~Hz}, 1 \mathrm{H}), 4.47(\mathrm{~d}, \mathrm{~J}=5.9 \mathrm{~Hz}, 2 \mathrm{H}) .{ }^{13} \mathbf{C} \mathbf{N M R}\left(101 \mathrm{MHz}, \mathrm{CDCl}_{3}\right) \delta$ 165.4, 136.6, 133.4, 130.4, 129.2, 128.8, 127.0, 42.9. ESI-HR calcd for $\mathrm{C}_{10} \mathrm{H}_{11} \mathrm{ClNO}^{+}\left([\mathrm{M}+\mathrm{H}]^{+}\right)$196.0524, found 196.0521. IR $v\left(\mathrm{~cm}^{-1}\right) 3281,3047,3008,2961,1651,1620,1536,751$. M. P. $105^{\circ} \mathrm{C}$. 
<smiles>C=CC(=O)NCCc1cc(Cl)ccc1Cl</smiles>

1k: Following the general procedure, 1k was obtained and purification by silica gel chromatography in $736.8 \mathrm{mg}$ (30\% yield) as a white solid. ${ }^{1} \mathbf{H}$ NMR $\left(400 \mathrm{MHz}, \mathrm{CDCl}_{3}\right) \delta 7.29(\mathrm{~d}, \mathrm{~J}=8.5 \mathrm{~Hz}, 1 \mathrm{H})$, $7.23(\mathrm{~d}, \mathrm{~J}=2.5 \mathrm{~Hz}, 1 \mathrm{H}), 7.16(\mathrm{dd}, \mathrm{J}=8.5,2.5 \mathrm{~Hz}, 1 \mathrm{H}), 6.27(\mathrm{dd}, \mathrm{J}=17.0,1.4 \mathrm{~Hz}, 1 \mathrm{H}), 6.06(\mathrm{dd}, \mathrm{J}$ $=17.0,10.3 \mathrm{~Hz}, 1 \mathrm{H}), 5.72(\mathrm{~s}, 1 \mathrm{H}), 5.65(\mathrm{dd}, \mathrm{J}=10.3,1.4 \mathrm{~Hz}, 1 \mathrm{H}), 3.59(\mathrm{dd}, \mathrm{J}=13.2,6.9 \mathrm{~Hz}, 2 \mathrm{H})$, $2.97(\mathrm{t}, \mathrm{J}=7.0 \mathrm{~Hz}, 2 \mathrm{H}) .{ }^{13} \mathbf{C}$ NMR $\left(101 \mathrm{MHz}, \mathrm{CDCl}_{3}\right) \delta 165.6,138.3,132.7,132.4,130.8,130.7$, 130.6, 128.1, 126.6, 39.0, 33.3. ESI-HR calcd for $\mathrm{C}_{11} \mathrm{H}_{12} \mathrm{Cl}_{2} \mathrm{NO}^{+}\left([\mathrm{M}+\mathrm{H}]^{+}\right)$244.0290, found 244.0286. IR $v\left(\mathrm{~cm}^{-1}\right) 3252,3074,2973,2945,1651,1622,1587,1467,750$. M. P. $124{ }^{\circ} \mathrm{C}$.<smiles>C=CC(=O)NCCc1cccs1</smiles>

11: Following the general procedure, $\mathbf{1 l}$ was obtained and purification by silica gel chromatography in $753.3 \mathrm{mg}$ (42\% yield) as a white solid. ${ }^{1} \mathbf{H}$ NMR $\left(400 \mathrm{MHz}, \mathrm{CDCl}_{3}\right) \delta 7.11(\mathrm{dd}, \mathrm{J}=5.1,1.0 \mathrm{~Hz}$, 1H), $6.90(\mathrm{dd}, \mathrm{J}=5.0,3.5 \mathrm{~Hz}, 1 \mathrm{H}), 6.80(\mathrm{~d}, \mathrm{~J}=2.7 \mathrm{~Hz}, 1 \mathrm{H}), 6.63(\mathrm{~s}, 1 \mathrm{H}), 6.22(\mathrm{dd}, \mathrm{J}=17.0,1.7 \mathrm{~Hz}$, 1H), $6.12(\mathrm{dd}, \mathrm{J}=17.0,10.0 \mathrm{~Hz}, 1 \mathrm{H}), 5.58(\mathrm{dd}, \mathrm{J}=10.0,1.7 \mathrm{~Hz}, 1 \mathrm{H}), 3.55(\mathrm{dd}, \mathrm{J}=13.0,6.8 \mathrm{~Hz}$, 2H), $3.04(\mathrm{t}, \mathrm{J}=6.9 \mathrm{~Hz}, 2 \mathrm{H}) .{ }^{13} \mathrm{C}$ NMR $\left(101 \mathrm{MHz}, \mathrm{CDCl}_{3}\right) \delta 165.7,141.0,130.8,126.8,126.0$, 125.1, 123.7, 40.8, 29.6. ESI-HR calcd for $\mathrm{C}_{9} \mathrm{H}_{12} \mathrm{NOS}^{+}\left([\mathrm{M}+\mathrm{H}]^{+}\right)$182.0634, found 182.0632. IR $v$ $\left(\mathrm{cm}^{-1}\right) 3274,3072,3005,2991,2929,1654,1625,1546,750$. M. P. $43{ }^{\circ} \mathrm{C}$. 


\section{Characterization of products:}

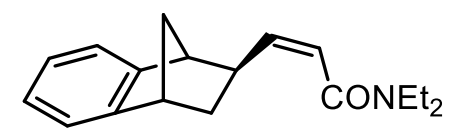

3aa: Following the general procedure, 1a $(12.7 \mathrm{mg}, 0.100 \mathrm{mmol})$ and $\mathbf{2 a}(28.5 \mathrm{mg}, 0.200 \mathrm{mmol})$ were converted to the chiral hydroalkenylation product. Purification by silica gel chromatography gave $26.4 \mathrm{mg}$ (98\% yield) of 3aa as a light yellow oil. ${ }^{1} \mathbf{H}$ NMR $\left(400 \mathrm{MHz}, \mathrm{CDCl}_{3}\right) \delta 7.23-7.09(\mathrm{~m}$, 2H), 7.09-6.98 (m, 2H), $5.99(\mathrm{~d}, \mathrm{~J}=11.5 \mathrm{~Hz}, 1 \mathrm{H}), 5.94(\mathrm{dd}, \mathrm{J}=11.4,9.1 \mathrm{~Hz}, 1 \mathrm{H}), 3.40-3.31(\mathrm{~m}$, 5H), $3.17(\mathrm{~d}, \mathrm{~J}=11.4 \mathrm{~Hz}, 1 \mathrm{H}), 2.79(\mathrm{td}, \mathrm{J}=8.4,4.7 \mathrm{~Hz}, 1 \mathrm{H}), 1.79(\mathrm{~m}, 1 \mathrm{H}), 1.77-1.72(\mathrm{~m}, 1 \mathrm{H}), 1.71-$ $1.64(\mathrm{~m}, 1 \mathrm{H}), 1.64-1.57(\mathrm{~m}, 1 \mathrm{H}), 1.15(\mathrm{t}, \mathrm{J}=7.1 \mathrm{~Hz}, 3 \mathrm{H}), 1.09(\mathrm{t}, \mathrm{J}=7.1 \mathrm{~Hz}, 3 \mathrm{H}) .{ }^{13} \mathbf{C}$ NMR $(101$ $\left.\mathrm{MHz}, \mathrm{CDCl}_{3}\right) \delta 166.9,148.1,147.6,145.0,125.6,125.6,121.3,120.8,120.6,49.8,46.2,44.0,42.4$, 39.6, 39.3, 36.2, 14.3, 13.1. ESI-HR calcd for $\mathrm{C}_{18} \mathrm{H}_{24} \mathrm{NO}^{+}\left([\mathrm{M}+\mathrm{H}]^{+}\right) 270.1852$, found 270.1852. IR $v\left(\mathrm{~cm}^{-1}\right) 3043,3019,2967,2870,1643,1626,751 .[\alpha]^{25} \mathrm{D}=+66.1\left(\mathrm{c}=1.325, \mathrm{CHCl}_{3}\right)$ for a $98 \%$ ee sample. The enantiomeric purity of this compound was determined by HPLC analysis in comparison with authentic racemic material (Chiralpak OJ-H, 95:5 hexanes:i-PrOH, $0.8 \mathrm{~mL} / \mathrm{min}, 254 \mathrm{~nm}$ ): $\mathrm{t}_{\text {major }}$ $=9.177 \mathrm{~min}, \mathrm{t}_{\operatorname{minor}}=11.355 \mathrm{~min}$.

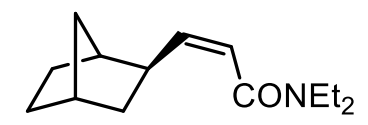

3ab: Following the general procedure, $\mathbf{1 a}(12.7 \mathrm{mg}, 0.100 \mathrm{mmol})$ and $\mathbf{2 b}(47.1 \mathrm{mg}, 0.500 \mathrm{mmol})$ were converted to the chiral hydroalkenylation product. Purification by silica gel chromatography gave $20.5 \mathrm{mg}$ (93\% yield) of $\mathbf{3 a b}$ as a light yellow oil. ${ }^{1} \mathbf{H} \mathbf{~ N M R}\left(400 \mathrm{MHz}, \mathrm{CDCl}_{3}\right) \delta 5.82(\mathrm{~d}, \mathrm{~J}=$ 11.6 Hz, 1H), $5.72(\mathrm{dd}, \mathrm{J}=11.5,9.8 \mathrm{~Hz}, 1 \mathrm{H}), 3.41(\mathrm{q}, \mathrm{J}=7.1 \mathrm{~Hz}, 2 \mathrm{H}), 3.33(\mathrm{q}, \mathrm{J}=7.2 \mathrm{~Hz}, 2 \mathrm{H}), 2.77$ (td, J = 9.2, 4.8 Hz, 1H), $2.23(\mathrm{~s}, 1 \mathrm{H}), 2.03(\mathrm{~d}, \mathrm{~J}=1.4 \mathrm{~Hz}, 1 \mathrm{H}), 1.69-1.58(\mathrm{~m}, 1 \mathrm{H}), 1.50-1.44(\mathrm{~m}$, 2H), 1.41-1.35 (m, 1H), 1.32-1.24 (m, 1H), 1.23-1.19 (m, 1H), 1.17-1.10 (m, 8H). ${ }^{13}$ C NMR (101 $\left.\mathrm{MHz}, \mathrm{CDCl}_{3}\right) \delta 167.2,146.5,119.2,42.6,42.4,41.3,39.4,38.7,36.5,35.9,29.6,28.7,14.3,13.1$. ESI-HR calcd for $\mathrm{C}_{14} \mathrm{H}_{24} \mathrm{NO}^{+}\left([\mathrm{M}+\mathrm{H}]^{+}\right)$222.1852, found 222.1849. IR $v\left(\mathrm{~cm}^{-1}\right) 3019,2949,1644$, 1623, 750. $[\alpha]^{25} \mathrm{D}=+46.9\left(\mathrm{c}=0.925, \mathrm{CHCl}_{3}\right)$ for a $99 \%$ ee sample. The enantiomeric purity of this compound was determined by HPLC analysis in comparison with authentic racemic material (Chiralpak OJ-H, 95:5 hexanes:i-PrOH, $0.5 \mathrm{~mL} / \mathrm{min}, 210 \mathrm{~nm}$ ): $\mathrm{t}_{\text {major }}=14.598 \mathrm{~min}, \mathrm{t}_{\text {minor }}=10.797$ 
$\min$.

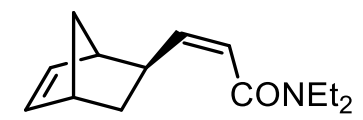

3ac: Following the general procedure, 1a $(12.7 \mathrm{mg}, 0.100 \mathrm{mmol})$ and $\mathbf{2 c}(46.1 \mathrm{mg}, 0.500 \mathrm{mmol})$ were converted to the chiral hydroalkenylation product. Purification by silica gel chromatography gave $19.9 \mathrm{mg}$ (91\% yield) of $\mathbf{3 a c}$ as a yellow oil. ${ }^{1} \mathbf{H}$ NMR $\left(400 \mathrm{MHz}, \mathrm{CDCl}_{3}\right) \delta 6.11(\mathrm{dd}, \mathrm{J}=5.6$, $3.0 \mathrm{~Hz}, 1 \mathrm{H}), 6.05(\mathrm{dd}, \mathrm{J}=5.6,2.9 \mathrm{~Hz}, 1 \mathrm{H}), 5.94(\mathrm{~d}, \mathrm{~J}=11.5 \mathrm{~Hz}, 1 \mathrm{H}), 5.82(\mathrm{dd}, \mathrm{J}=11.4,10.2 \mathrm{~Hz}$, 1H), 3.43-3.31 (m, 4H), $2.87(\mathrm{~s}, 1 \mathrm{H}), 2.74-2.60(\mathrm{~m}, 2 \mathrm{H}), 1.50-1.42(\mathrm{~m}, 1 \mathrm{H}), 1.39(\mathrm{~d}, \mathrm{~J}=8.6 \mathrm{~Hz}$, 1H), 1.36-1.27 (m, 2H), $1.13(\mathrm{~m}, 6 \mathrm{H}) .{ }^{13} \mathrm{C}$ NMR (101 MHz, $\left.\mathrm{CDCl}_{3}\right) \delta$ 167.1, 146.1, 136.9, 136.2, 120.8, 48.2, 45.3, 42.4, 42.2, 39.4, 37.8, 33.8, 14.3, 13.1. ESI-HR calcd for $\mathrm{C}_{14} \mathrm{H}_{22} \mathrm{NO}^{+}\left([\mathrm{M}+\mathrm{H}]^{+}\right)$ 220.1696, found 220.1693. IR $v\left(\mathrm{~cm}^{-1}\right) 3059,2969,2935,2870,1644,1626,750 .[\alpha]^{25} \mathrm{D}=+60.0(\mathrm{c}$ $=0.830, \mathrm{CHCl}_{3}$ ) for a $94 \%$ ee sample. The enantiomeric purity of this compound was determined by HPLC analysis in comparison with authentic racemic material (Chiralpak OD-H, 97:3 hexanes:i$\operatorname{PrOH}, 0.5 \mathrm{~mL} / \mathrm{min}, 210 \mathrm{~nm}$ ): $\mathrm{t}_{\text {major }}=17.083 \mathrm{~min}, \mathrm{t}_{\mathrm{minor}}=18.849 \mathrm{~min}$.

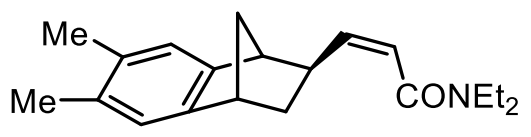

3ad: Following the general procedure, $\mathbf{1 a}(12.7 \mathrm{mg}, 0.100 \mathrm{mmol})$ and $\mathbf{2 d}(34.0 \mathrm{mg}, 0.200 \mathrm{mmol})$ were converted to the chiral hydroalkenylation product. Purification by silica gel chromatography gave $29.4 \mathrm{mg}$ (99\% yield) of 3ad as a light yellow oil. ${ }^{1} \mathbf{H} \mathbf{~ N M R}\left(400 \mathrm{MHz}, \mathrm{CDCl}_{3}\right) \delta 6.99(\mathrm{~s}, 1 \mathrm{H})$, $6.94(\mathrm{~s}, 1 \mathrm{H}), 5.97(\mathrm{~d}, \mathrm{~J}=11.5 \mathrm{~Hz}, 1 \mathrm{H}), 5.94-5.82(\mathrm{~m}, 1 \mathrm{H}), 3.43-3.31(\mathrm{~m}, 4 \mathrm{H}), 3.30(\mathrm{~s}, 1 \mathrm{H}), 3.11(\mathrm{~s}$, 1H), $2.75(\mathrm{td}, \mathrm{J}=8.7,4.9 \mathrm{~Hz}, 1 \mathrm{H}), 2.20(\mathrm{~s}, 6 \mathrm{H}), 1.77(\mathrm{~d}, \mathrm{~J}=9.1 \mathrm{~Hz}, 1 \mathrm{H}), 1.70(\mathrm{~d}, \mathrm{~J}=9.1 \mathrm{~Hz}, 1 \mathrm{H})$, $1.66-1.55(\mathrm{~m}, 2 \mathrm{H}), 1.12(\mathrm{~m}, 6 \mathrm{H}) .{ }^{13} \mathbf{C}$ NMR $\left(101 \mathrm{MHz}, \mathrm{CDCl}_{3}\right) \delta 167.0,145.8,145.4,145.0,133.3$, $133.3,122.3,122.1,121.2,49.6,46.2,43.7,42.4,40.0,39.3,36.5,19.7,19.8,14.3,13.1$. ESI-HR calcd for $\mathrm{C}_{20} \mathrm{H}_{28} \mathrm{NO}^{+}\left([\mathrm{M}+\mathrm{H}]^{+}\right)$298.2165, found 298.2152. IR $v\left(\mathrm{~cm}^{-1}\right)$ 3005, 2965, 2932, 2868, $1644,1622,750 .[\alpha]^{25} \mathrm{D}=+56.9\left(\mathrm{c}=1.165, \mathrm{CHCl}_{3}\right)$ for a $98 \%$ ee sample. The enantiomeric purity of this compound was determined by HPLC analysis in comparison with authentic racemic material (Chiralpak OD-H, 97:3 hexanes:i-PrOH, $0.5 \mathrm{~mL} / \mathrm{min}, 254 \mathrm{~nm}$ ): $\mathrm{t}_{\text {major }}=19.185 \mathrm{~min}, \mathrm{t}_{\mathrm{minor}}=21.706$ min. 


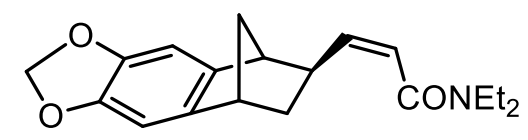

3ae: Following the general procedure, 1a $(12.7 \mathrm{mg}, 0.100 \mathrm{mmol})$ and $\mathbf{2 e}(37.2 \mathrm{mg}, 0.200 \mathrm{mmol})$ were converted to the chiral hydroalkenylation product. Purification by silica gel chromatography gave $30.9 \mathrm{mg}\left(99 \%\right.$ yield) of 3ae as a light yellow oil. ${ }^{\mathbf{1}} \mathbf{H} \mathbf{N M R}\left(400 \mathrm{MHz}, \mathrm{CDCl}_{3}\right) \delta 6.72(\mathrm{~s}, 1 \mathrm{H})$, $6.68(\mathrm{~s}, 1 \mathrm{H}), 5.98(\mathrm{~d}, \mathrm{~J}=11.5 \mathrm{~Hz}, 1 \mathrm{H}), 5.95-5.86(\mathrm{~m}, 2 \mathrm{H}), 5.82(\mathrm{~s}, 1 \mathrm{H}), 3.41-3.30(\mathrm{~m}, 4 \mathrm{H}), 3.27(\mathrm{~s}$, 1H), $3.06(\mathrm{~s}, 1 \mathrm{H}), 2.76(\mathrm{td}, \mathrm{J}=8.8,4.7 \mathrm{~Hz}, 1 \mathrm{H}), 1.72(\mathrm{q}, \mathrm{J}=9.1 \mathrm{~Hz}, 2 \mathrm{H}), 1.66-1.51(\mathrm{~m}, 2 \mathrm{H}), 1.12$ (m, 6H). $\left.{ }^{13} \mathbf{C ~ N M R ~ ( 1 0 1 ~ M H z , ~} \mathrm{CDCl}_{3}\right) \delta 166.8,145.3,145.2,145.1,141.4,140.9,121.2,103.2$, 103.0, 100.4, 49.9, 46.4, 44.1, 42.4, 39.9, 39.3, 36.4, 14.3, 13.1. ESI-HR calcd for $\mathrm{C}_{19} \mathrm{H}_{24} \mathrm{NO}_{3}{ }^{+}$ $\left([\mathrm{M}+\mathrm{H}]^{+}\right) 314.1751$, found 314.1742. IR $v\left(\mathrm{~cm}^{-1}\right) 3005,2967,2871,1643,1622,750 .[\alpha]^{25} \mathrm{D}=+67.3$ $\left(\mathrm{c}=1.280, \mathrm{CHCl}_{3}\right)$ for a $97 \%$ ee sample. The enantiomeric purity of this compound was determined by HPLC analysis in comparison with authentic racemic material (Chiralpak OJ-H, 95:5 hexanes:i$\mathrm{PrOH}, 0.8 \mathrm{~mL} / \mathrm{min}, 254 \mathrm{~nm}): \mathrm{t}_{\text {major }}=13.319 \mathrm{~min}, \mathrm{t}_{\text {minor }}=17.174 \mathrm{~min}$.

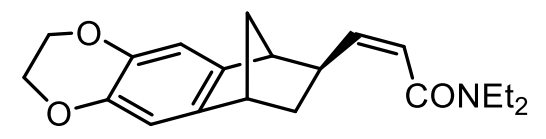

3af: Following the general procedure, $\mathbf{1 a}(12.7 \mathrm{mg}, 0.100 \mathrm{mmol})$ and $\mathbf{2 f}(40.0 \mathrm{mg}, 0.200 \mathrm{mmol})$ were converted to the chiral hydroalkenylation product. Purification by silica gel chromatography gave $32.5 \mathrm{mg}$ (99\% yield) of 3af as a light yellow oil. ${ }^{\mathbf{1}} \mathbf{H} \mathbf{N M R}\left(400 \mathrm{MHz}, \mathrm{CDCl}_{3}\right) \delta 6.71(\mathrm{~s}, 1 \mathrm{H})$, $6.66(\mathrm{~s}, 1 \mathrm{H}), 5.97(\mathrm{~d}, \mathrm{~J}=11.5 \mathrm{~Hz}, 1 \mathrm{H}), 5.93-5.86(\mathrm{~m}, 1 \mathrm{H}), 4.19(\mathrm{~s}, 4 \mathrm{H}), 3.44-3.31(\mathrm{~m}, 4 \mathrm{H}), 3.25(\mathrm{~s}$, 1H), $3.05(\mathrm{~s}, 1 \mathrm{H}), 2.77(\mathrm{td}, \mathrm{J}=8.9,4.5 \mathrm{~Hz}, 1 \mathrm{H}), 1.75(\mathrm{~d}, \mathrm{~J}=9.1 \mathrm{~Hz}, 1 \mathrm{H}), 1.71-1.60(\mathrm{~m}, 2 \mathrm{H}), 1.55$ $(\mathrm{m}, 1 \mathrm{H}), 1.13(\mathrm{~m}, 6 \mathrm{H}) .{ }^{13} \mathrm{C} \mathbf{N M R}\left(101 \mathrm{MHz}, \mathrm{CDCl}_{3}\right) \delta 166.9,145.2,141.2,141.2,141.1,140.7$, 121.1, 110.4, 110.3, 64.34, 64.3, 49.4, 46.0, 43. 7, 42.4, 40.1, 39.3, 36.7, 14.3, 13.1. ESI-HR calcd for $\mathrm{C}_{20} \mathrm{H}_{26} \mathrm{NO}_{3}{ }^{+}\left([\mathrm{M}+\mathrm{H}]^{+}\right)$328.1907, found 328.1899. IR $v\left(\mathrm{~cm}^{-1}\right) 3004,2968,2871,1643,1622$, 750. $[\alpha]^{25} \mathrm{D}=+64.3\left(\mathrm{c}=1.385, \mathrm{CHCl}_{3}\right)$ for a $97 \%$ ee sample. The enantiomeric purity of this compound was determined by HPLC analysis in comparison with authentic racemic material 
(Chiralpak OJ-H, 95:5 hexanes:i-PrOH, $0.8 \mathrm{~mL} / \mathrm{min}, 254 \mathrm{~nm}$ ): $\mathrm{t}_{\text {major }}=19.954 \mathrm{~min}, \mathrm{t}_{\text {minor }}=26.326$ $\min$.

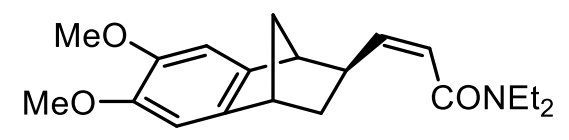

3ag: Following the general procedure, 1a $(12.7 \mathrm{mg}, 0.100 \mathrm{mmol})$ and $\mathbf{2 g}(40.4 \mathrm{mg}, 0.200 \mathrm{mmol})$ were converted to the chiral hydroalkenylation product. Purification by silica gel chromatography gave $32.7 \mathrm{mg}$ (99\% yield) of $\mathbf{3 a g}$ as a yellow oil. ${ }^{1} \mathbf{H}$ NMR (400 MHz, $\left.\mathrm{CDCl}_{3}\right) \delta 6.82(\mathrm{~s}, 1 \mathrm{H}), 6.77$ (s, 1H), $5.97(\mathrm{~d}, \mathrm{~J}=11.5 \mathrm{~Hz}, 1 \mathrm{H}), 5.94-5.87(\mathrm{~m}, 1 \mathrm{H}), 3.83(\mathrm{~s}, 6 \mathrm{H}), 3.35(\mathrm{~m}, 5 \mathrm{H}), 3.11(\mathrm{~s}, 1 \mathrm{H}), 2.79$ $2.68(\mathrm{~m}, 1 \mathrm{H}), 1.73(\mathrm{~m}, 2 \mathrm{H}), 1.65-1.52(\mathrm{~m}, 2 \mathrm{H}), 1.19-1.04(\mathrm{~m}, 6 \mathrm{H}) .{ }^{13} \mathbf{C ~ N M R}\left(101 \mathrm{MHz}, \mathrm{CDCl}_{3}\right) \delta$ 166.9, 147.1, 147.1, 145.2, 140.2, 139.8, 121.1, 105.9, 105.8, 56.2, 56.2, 50.0, 46.4, 44.1, 42.4, 40.1, 39.3, 36.6, 14.3, 13.1. ESI-HR calcd for $\mathrm{C}_{20} \mathrm{H}_{28} \mathrm{NO}_{3}{ }^{+}\left([\mathrm{M}+\mathrm{H}]^{+}\right) 330.2064$, found 330.2054. IR v $\left(\mathrm{cm}^{-1}\right) 3004,2964,2868,1643,1623,750 .[\alpha]^{25} \mathrm{D}=+55.3\left(\mathrm{c}=1.560, \mathrm{CHCl}_{3}\right)$ for a $97 \%$ ee sample. The enantiomeric purity of this compound was determined by HPLC analysis in comparison with authentic racemic material (Chiralpak OJ-H, 92:8 hexanes:i-PrOH, $0.8 \mathrm{~mL} / \mathrm{min}, 254 \mathrm{~nm}$ ): $\mathrm{t}_{\text {major }}=$ $12.153 \mathrm{~min}, \mathrm{t}_{\mathrm{minor}}=14.414 \mathrm{~min}$.

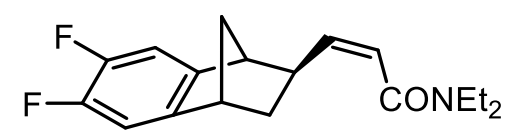

3ah: Following the general procedure, $\mathbf{1 a}(12.7 \mathrm{mg}, 0.100 \mathrm{mmol})$ and $\mathbf{2 h}(35.6 \mathrm{mg}, 0.200 \mathrm{mmol})$ were converted to the chiral hydroalkenylation product. Purification by silica gel chromatography gave $25.9 \mathrm{mg}$ (85\% yield) of 3ah as a yellow oil. ${ }^{1} \mathbf{H} \mathbf{~ N M R}\left(400 \mathrm{MHz}, \mathrm{CDCl}_{3}\right) \delta 6.99(\mathrm{t}, \mathrm{J}=8.5 \mathrm{~Hz}$, 1H), $6.93(\mathrm{t}, \mathrm{J}=8.5 \mathrm{~Hz}, 1 \mathrm{H}), 6.01(\mathrm{~d}, \mathrm{~J}=11.5 \mathrm{~Hz}, 1 \mathrm{H}), 5.96-5.86(\mathrm{~m}, 1 \mathrm{H}), 3.42-3.28(\mathrm{~m}, 5 \mathrm{H}), 3.12$ (s, 1H), 2.89-2.76 (m, 1H), $1.76(\mathrm{q}, \mathrm{J}=9.3 \mathrm{~Hz}, 2 \mathrm{H}), 1.67-1.56(\mathrm{~m}, 2 \mathrm{H}), 1.12(\mathrm{~m}, 6 \mathrm{H}) .{ }^{13} \mathrm{C}$ NMR $\left(101 \mathrm{MHz}, \mathrm{CDCl}_{3}\right) \delta 166.6(\mathrm{~s}), 149.8(\mathrm{dd}, \mathrm{J}=14.9,9.4 \mathrm{~Hz}), 147.3(\mathrm{dd}, \mathrm{J}=14.9,9.4 \mathrm{~Hz}), 145.1(\mathrm{~s})$, 143.9 (t, J = 4.8 Hz), 143.5 (s), 121.4 (s), 110.6-110.0 (m, 2C), 49.6 (s), 46.5 (s), 43.8 (s), 42.4 (s), 39.5 (s), 39.5 (s), 36.1 (s), 14.3 (s), 13.1 (s). ESI-HR calcd for $\mathrm{C}_{18} \mathrm{H}_{22} \mathrm{~F}_{2} \mathrm{NO}^{+}\left([\mathrm{M}+\mathrm{H}]^{+}\right)$306.1664, found 306.1656. IR $v\left(\mathrm{~cm}^{-1}\right) 3004,2974,2873,1644,1615,750 .[\alpha]^{25} \mathrm{D}=+63.6\left(\mathrm{c}=1.270, \mathrm{CHCl}_{3}\right)$ for a $97 \%$ ee sample. The enantiomeric purity of this compound was determined by HPLC analysis 
in comparison with authentic racemic material (Chiralpak OD-H, 95:5 hexanes:i-PrOH, $0.8 \mathrm{~mL} / \mathrm{min}$, $254 \mathrm{~nm}): \mathrm{t}_{\text {major }}=11.539 \mathrm{~min}, \mathrm{t}_{\mathrm{minor}}=12.993 \mathrm{~min}$.

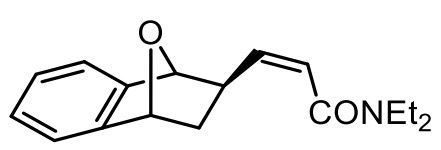

3ai: Following the general procedure, $\mathbf{1 a}(12.7 \mathrm{mg}, 0.100 \mathrm{mmol})$ and $\mathbf{2 i}(28.8 \mathrm{mg}, 0.200 \mathrm{mmol})$ were converted to the chiral hydroalkenylation product. Purification by silica gel chromatography gave $25.7 \mathrm{mg}$ (95\% yield) of 3ai as a yellow oil. ${ }^{1} \mathbf{H} \mathbf{~ N M R}\left(400 \mathrm{MHz}, \mathrm{CDCl}_{3}\right) \delta 7.31-7.27(\mathrm{~m}, 1 \mathrm{H}), 7.23-$ $7.18(\mathrm{~m}, 1 \mathrm{H}), 7.16-7.10(\mathrm{~m}, 2 \mathrm{H}), 6.11-6.03(\mathrm{~m}, 2 \mathrm{H}), 5.43(\mathrm{~d}, \mathrm{~J}=4.6 \mathrm{~Hz}, 1 \mathrm{H}), 5.14(\mathrm{~s}, 1 \mathrm{H}), 3.45-$ $3.28(\mathrm{~m}, 4 \mathrm{H}), 3.26-3.14(\mathrm{~m}, 1 \mathrm{H}), 1.89-1.76(\mathrm{~m}, 2 \mathrm{H}), 1.17(\mathrm{t}, \mathrm{J}=7.1 \mathrm{~Hz}, 3 \mathrm{H}), 1.10(\mathrm{t}, \mathrm{J}=7.1 \mathrm{~Hz}$, 3H). ${ }^{13} \mathrm{C}$ NMR $\left(101 \mathrm{MHz}, \mathrm{CDCl}_{3}\right) \delta 166.4,145.8,145.2,144.8,126.6,121.0,119.3,118.7,84.0$, 79.4, 42.4, 39.8, 39.6, 35.9, 29.7, 14.4, 13.1. ESI-HR calcd for $\mathrm{C}_{17} \mathrm{H}_{22} \mathrm{NO}_{2}{ }^{+}\left([\mathrm{M}+\mathrm{H}]^{+}\right) 272.1645$, found 272.1639. IR $v\left(\mathrm{~cm}^{-1}\right) 3003,2976,2935,2872,1645,1621,751 .[\alpha]^{25} \mathrm{D}=+9.6(\mathrm{c}=1.100$, $\mathrm{CHCl}_{3}$ ) for a $29 \%$ ee sample. The enantiomeric purity of this compound was determined by HPLC analysis in comparison with authentic racemic material (Chiralpak OJ-H, 95:5 hexanes:i-PrOH, 0.8 $\mathrm{mL} / \mathrm{min}, 254 \mathrm{~nm}$ ): $\mathrm{t}_{\text {major }}=16.265 \mathrm{~min}, \mathrm{t}_{\mathrm{minor}}=20.648 \mathrm{~min}$.

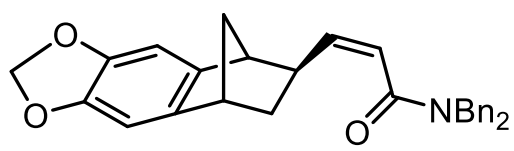

3be: Following the general procedure, $\mathbf{1 b}(25.1 \mathrm{mg}, 0.100 \mathrm{mmol})$ and $\mathbf{2 e}(37.2 \mathrm{mg}, 0.200 \mathrm{mmol})$ were converted to the chiral hydroalkenylation product. Purification by silica gel chromatography gave $38.9 \mathrm{mg}$ (89\% yield) of 3be as a white solid. ${ }^{1} \mathbf{H} \mathbf{~ N M R}\left(400 \mathrm{MHz}, \mathrm{CDCl}_{3}\right) \delta 7.37$ (t, J = 7.2 $\mathrm{Hz}, 2 \mathrm{H}), 7.34-7.26(\mathrm{~m}, 4 \mathrm{H}), 7.18(\mathrm{dd}, \mathrm{J}=12.0,5.1 \mathrm{~Hz}, 4 \mathrm{H}), 6.74(\mathrm{~d}, \mathrm{~J}=13.2 \mathrm{~Hz}, 2 \mathrm{H}), 6.09(\mathrm{~d}, \mathrm{~J}=$ $11.4 \mathrm{~Hz}, 1 \mathrm{H}), 6.02-5.94(\mathrm{~m}, 1 \mathrm{H}), 5.90(\mathrm{dd}, \mathrm{J}=14.2,1.4 \mathrm{~Hz}, 2 \mathrm{H}), 4.61(\mathrm{~d}, \mathrm{~J}=14.7 \mathrm{~Hz}, 1 \mathrm{H}), 4.56-$ $4.44(\mathrm{~m}, 3 \mathrm{H}), 3.30(\mathrm{~s}, 1 \mathrm{H}), 3.08(\mathrm{~s}, 1 \mathrm{H}), 2.88(\mathrm{td}, \mathrm{J}=9.4,4.6 \mathrm{~Hz}, 1 \mathrm{H}), 1.79-1.71(\mathrm{~m}, 2 \mathrm{H}), 1.68$ 1.55 (m, 2H). ${ }^{13} \mathrm{C}$ NMR $\left(101 \mathrm{MHz}, \mathrm{CDCl}_{3}\right) \delta 168.1,146.5,145.3,145.2,141.3,140.8,137.2,136.5$, $128.8,128.5,128.3,127.6,127.4,126.8,120.8,103.2,103.1,100.4,50.4,49.9,47.1,46.4,44.1$, 40.3, 36.3. ESI-HR calcd for $\mathrm{C}_{29} \mathrm{H}_{28} \mathrm{NO}_{3}{ }^{+}\left([\mathrm{M}+\mathrm{H}]^{+}\right) 438.2064$, found 438.2054. IR $v\left(\mathrm{~cm}^{-1}\right) 3027$, 3006, 2964, 2870, $1642,1586,750$. M. P. $143{ }^{\circ} \mathrm{C} .[\alpha]^{25} \mathrm{D}=+74.0\left(\mathrm{c}=1.945, \mathrm{CHCl}_{3}\right)$ for a $96 \%$ ee sample. The enantiomeric purity of this compound was determined by HPLC analysis in comparison 
with authentic racemic material (Chiralpak OD-H, 98:2 hexanes:i-PrOH, $0.5 \mathrm{~mL} / \mathrm{min}, 254 \mathrm{~nm}$ ): $\mathrm{t}_{\text {major }}=63.270 \mathrm{~min}, \mathrm{t}_{\mathrm{minor}}=68.426 \mathrm{~min}$.

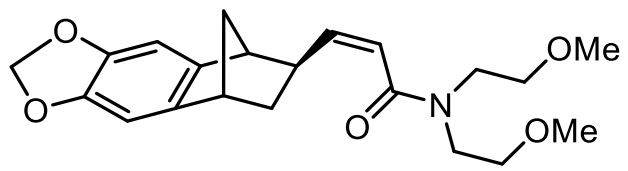

3ce: Following the general procedure, $\mathbf{1 c}(18.7 \mathrm{mg}, 0.100 \mathrm{mmol})$ and $\mathbf{2 e}(37.2 \mathrm{mg}, 0.200 \mathrm{mmol})$ were converted to the chiral hydroalkenylation product. Purification by silica gel chromatography gave $28.7 \mathrm{mg}$ (77\% yield) of 3ce as a yellow oil. ${ }^{1} \mathbf{H}$ NMR (400 MHz, $\left.\mathrm{CDCl}_{3}\right) \delta 6.73(\mathrm{~s}, 1 \mathrm{H}), 6.68$ $(\mathrm{s}, 1 \mathrm{H}), 6.06(\mathrm{~d}, \mathrm{~J}=11.5 \mathrm{~Hz}, 1 \mathrm{H}), 5.96-5.89(\mathrm{~m}, 1 \mathrm{H}), 5.84(\mathrm{dd}, \mathrm{J}=22.8,1.1 \mathrm{~Hz}, 2 \mathrm{H}), 3.61-3.53(\mathrm{~m}$, 4H), 3.51-3.44 (m, 4H), $3.33(\mathrm{~s}, 3 \mathrm{H}), 3.27(\mathrm{~s}, 1 \mathrm{H}), 3.23(\mathrm{~s}, 3 \mathrm{H}), 3.06(\mathrm{~s}, 1 \mathrm{H}), 2.73(\mathrm{td}, \mathrm{J}=9.1,5.1$ $\mathrm{Hz}, 1 \mathrm{H}), 1.76-1.69(\mathrm{~m}, 2 \mathrm{H}), 1.62-1.52(\mathrm{~m}, 2 \mathrm{H}) .{ }^{13} \mathbf{C} \mathbf{N M R}\left(101 \mathrm{MHz}, \mathrm{CDCl}_{3}\right) \delta 168.1,145.4,145.2$, 145.1, 141.4, 141.0, 121.3, 103.2, 103.1, 100.4, 71.1, 70.8, 59.0, 58.7, 49.8, 49.2, 46.4, 45.7, 44.1, 40.0, 36.4. ESI-HR calcd for $\mathrm{C}_{21} \mathrm{H}_{28} \mathrm{NO}_{5}{ }^{+}\left([\mathrm{M}+\mathrm{H}]^{+}\right)$374.1962, found 374.1954. IR $v\left(\mathrm{~cm}^{-1}\right)$ 3004, $2963,2931,2873,1644,1623,750 .[\alpha]^{25} \mathrm{D}=+52.7\left(\mathrm{c}=1.295, \mathrm{CHCl}_{3}\right)$ for a $96 \%$ ee sample. The enantiomeric purity of this compound was determined by HPLC analysis in comparison with authentic racemic material (Chiralpak OJ-H, $92: 8$ hexanes:i-PrOH, $0.8 \mathrm{~mL} / \mathrm{min}, 254 \mathrm{~nm}$ ): $\mathrm{t}_{\text {major }}=$ $11.518 \mathrm{~min}, \mathrm{t}_{\mathrm{minor}}=16.723 \mathrm{~min}$.

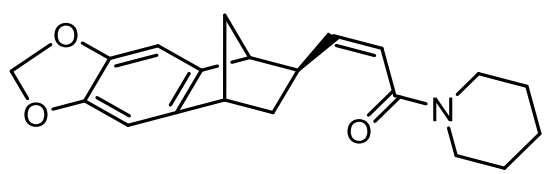

3de: Following the general procedure, $1 \mathbf{1 d}(13.9 \mathrm{mg}, 0.100 \mathrm{mmol})$ and $\mathbf{2 e}(37.2 \mathrm{mg}, 0.200 \mathrm{mmol})$ were converted to the chiral hydroalkenylation product. Purification by silica gel chromatography gave $24.9 \mathrm{mg}$ (77\% yield) of $\mathbf{3 b e}$ as a white solid. ${ }^{1} \mathbf{H}$ NMR $\left(400 \mathrm{MHz}, \mathrm{CDCl}_{3}\right) \delta 6.71(\mathrm{~s}, 1 \mathrm{H}), 6.68$ (s, 1H), $5.93(\mathrm{~d}, \mathrm{~J}=11.5 \mathrm{~Hz}, 1 \mathrm{H}), 5.90-5.81(\mathrm{~m}, 3 \mathrm{H}), 3.60-3.37(\mathrm{~m}, 4 \mathrm{H}), 3.27(\mathrm{~s}, 1 \mathrm{H}), 3.06(\mathrm{~s}, 1 \mathrm{H})$, $2.56(\mathrm{dt}, \mathrm{J}=9.2,6.4 \mathrm{~Hz}, 1 \mathrm{H}), 1.73(\mathrm{q}, \mathrm{J}=9.1 \mathrm{~Hz}, 2 \mathrm{H}), 1.67-1.59(\mathrm{~m}, 2 \mathrm{H}), 1.58-1.48(\mathrm{~m}, 6 \mathrm{H}) .{ }^{13} \mathrm{C}$ NMR $\left(101 \mathrm{MHz}, \mathrm{CDCl}_{3}\right) \quad \delta 166.4,145.2,145.1,143.4,141.4,140.9,121.5,103.1,103.1,100.4$, 49.8, 47.4, 46.4, 44.1, 42.1, 40.1, 36.3, 26.6, 25.6, 24.6. ESI-HR calcd for $\mathrm{C}_{20} \mathrm{H}_{24} \mathrm{NO}_{3}{ }^{+}\left([\mathrm{M}+\mathrm{H}]^{+}\right)$ 326.1751, found 326.1744. IR $v\left(\mathrm{~cm}^{-1}\right) 3004,2938,2860,1640,1617,750$. M. P. $116^{\circ}$ C. EA calcd 
for $\mathrm{C}, 73.82 ; \mathrm{H}, 7.12 ; \mathrm{N}, 4.30 ;$ found $\mathrm{C}, 73.64 ; \mathrm{H}, 7.39 ; \mathrm{N}, 4.38 .[\alpha]^{25} \mathrm{D}=+62.9\left(\mathrm{c}=1.030, \mathrm{CHCl}_{3}\right)$ for a 94\% ee sample. The enantiomeric purity of this compound was determined by HPLC analysis in comparison with authentic racemic material (Chiralpak OD-H, 97:3 hexanes:i-PrOH, $0.5 \mathrm{~mL} / \mathrm{min}$, $254 \mathrm{~nm}): \mathrm{t}_{\text {major }}=45.114 \mathrm{~min}, \mathrm{t}_{\mathrm{minor}}=52.836 \mathrm{~min}$.

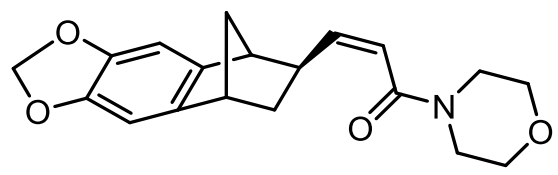

3ee: Following the general procedure, $\mathbf{1 e}(14.1 \mathrm{mg}, 0.100 \mathrm{mmol})$ and $\mathbf{2 e}(37.2 \mathrm{mg}, 0.200 \mathrm{mmol})$ were converted to the chiral hydroalkenylation product. Purification by silica gel chromatography gave $30.1 \mathrm{mg}$ (92\% yield) of 3be as a yellow oil. ${ }^{1} \mathbf{H}$ NMR $\left(400 \mathrm{MHz}, \mathrm{CDCl}_{3}\right) \delta 6.73(\mathrm{~s}, 1 \mathrm{H}), 6.69$ (s, 1H), 5.99-5.91 (m, 2H), $5.89(\mathrm{~d}, \mathrm{~J}=1.3 \mathrm{~Hz}, 1 \mathrm{H}), 5.84(\mathrm{~d}, \mathrm{~J}=1.3 \mathrm{~Hz}, 1 \mathrm{H}), 3.63(\mathrm{~d}, \mathrm{~J}=8.9 \mathrm{~Hz}$, $6 \mathrm{H}), 3.51(\mathrm{~d}, \mathrm{~J}=3.3 \mathrm{~Hz}, 2 \mathrm{H}), 3.29(\mathrm{~s}, 1 \mathrm{H}), 3.06(\mathrm{~s}, 1 \mathrm{H}), 2.63(\mathrm{td}, \mathrm{J}=8.3,5.3 \mathrm{~Hz}, 1 \mathrm{H}), 1.76-1.71$ $(\mathrm{m}, 2 \mathrm{H}), 1.56(\mathrm{td}, \mathrm{J}=7.3,3.7 \mathrm{~Hz}, 2 \mathrm{H}) .{ }^{13} \mathbf{C} \mathbf{~ N M R}\left(101 \mathrm{MHz}, \mathrm{CDCl}_{3}\right) \delta 166.5,145.5,145.3,145.2$, $141.3,140.7,120.2,103.1,103.1,100.4,66.9,66.8,49.8,46.7,46.4,44.1,41.6,40.3$, 36.3. ESIHR calcd for $\mathrm{C}_{19} \mathrm{H}_{22} \mathrm{NO}_{4}{ }^{+}\left([\mathrm{M}+\mathrm{H}]^{+}\right)$328.1543, found 328.1537. IR $v\left(\mathrm{~cm}^{-1}\right) 3005,2966,2864,1643$, $1621,750 .[\alpha]^{25} \mathrm{D}=+49.9\left(c=1.540, \mathrm{CHCl}_{3}\right)$ for a $98 \%$ ee sample. The enantiomeric purity of this compound was determined by HPLC analysis in comparison with authentic racemic material (Chiralpak OD-H, 95:5 hexanes:i-PrOH, $0.8 \mathrm{~mL} / \mathrm{min}, 254 \mathrm{~nm}$ ): $t_{\text {major }}=36.560 \mathrm{~min}, t_{\text {minor }}=34.206$ $\min$.

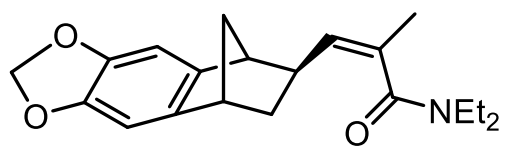

3fe: Following the general procedure, $\mathbf{1 f}(14.1 \mathrm{mg}, 0.100 \mathrm{mmol})$ and $\mathbf{2 e}(37.2 \mathrm{mg}, 0.200 \mathrm{mmol})$ were converted to the chiral hydroalkenylation product. Purification by silica gel chromatography gave $32.4 \mathrm{mg}$ (99\% yield) of $\mathbf{3 f e}$ as a yellow oil. ${ }^{1} \mathbf{H}$ NMR $\left(400 \mathrm{MHz}, \mathrm{CDCl}_{3}\right) \delta 6.67(\mathrm{~s}, 1 \mathrm{H}), 6.65(\mathrm{~s}, 1 \mathrm{H})$, $5.87(\mathrm{~d}, \mathrm{~J}=1.4 \mathrm{~Hz}, 1 \mathrm{H}), 5.84(\mathrm{~d}, \mathrm{~J}=1.4 \mathrm{~Hz}, 1 \mathrm{H}), 5.34(\mathrm{dd}, \mathrm{J}=10.5,1.4 \mathrm{~Hz}, 1 \mathrm{H}), 3.37(\mathrm{td}, \mathrm{J}=14.0$, $7.0 \mathrm{~Hz}, 4 \mathrm{H}), 3.25(\mathrm{~d}, \mathrm{~J}=1.6 \mathrm{~Hz}, 1 \mathrm{H}), 3.02(\mathrm{~s}, 1 \mathrm{H}), 2.10-2.01(\mathrm{~m}, 1 \mathrm{H}), 1.89(\mathrm{~d}, \mathrm{~J}=1.3 \mathrm{~Hz}, 3 \mathrm{H})$, 1.75-1.68 (m, 2H), $1.54(\mathrm{dt}, \mathrm{J}=12.0,4.0 \mathrm{~Hz}, 1 \mathrm{H}), 1.50-1.42(\mathrm{~m}, 1 \mathrm{H}), 1.14(\mathrm{t}, \mathrm{J}=6.9 \mathrm{~Hz}, 3 \mathrm{H}), 1.06$ $(\mathrm{t}, \mathrm{J}=7.1 \mathrm{~Hz}, 3 \mathrm{H}) .{ }^{13} \mathrm{C} \mathbf{N M R}\left(101 \mathrm{MHz}, \mathrm{CDCl}_{3}\right) \delta 171.2,145.1,145.1,141.5,141.1,132.7,131.7$, 
103.1, 102.8, 100.4, 49.9, 46.4, 44.0, 42.0, 40.3, 37.7, 36.0, 20.8, 14.4, 12.7. ESI-HR calcd for $\mathrm{C}_{20} \mathrm{H}_{26} \mathrm{NO}_{3}{ }^{+}\left([\mathrm{M}+\mathrm{H}]^{+}\right)$328.1907, found 328.1901. IR $v\left(\mathrm{~cm}^{-1}\right)$ 3005, 2976, 2875, 1641, 1609, 749, . $[\alpha]^{25} \mathrm{D}=+52.0\left(c=1.730, \mathrm{CHCl}_{3}\right)$ for a $94 \%$ ee sample. The enantiomeric purity of this compound was determined by HPLC analysis in comparison with authentic racemic material (Chiralpak ODH, 97:3 hexanes: $i-\mathrm{PrOH}, 0.8 \mathrm{~mL} / \mathrm{min}, 254 \mathrm{~nm}$ ): $t_{\text {major }}=14.457 \mathrm{~min}, t_{\operatorname{minor}}=13.462 \mathrm{~min}$.

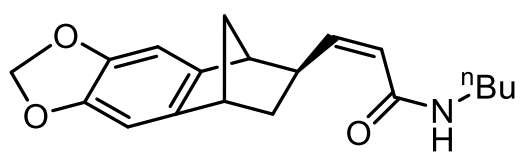

3ge: Following the general procedure, $\mathbf{1 g}(12.7 \mathrm{mg}, 0.100 \mathrm{mmol})$ and $\mathbf{2 e}(37.2 \mathrm{mg}, 0.200 \mathrm{mmol})$ were converted to the chiral hydroalkenylation product. Purification by silica gel chromatography gave $25.9 \mathrm{mg}$ (83\% yield) of 3ge as a white solid. ${ }^{1} \mathbf{H}$ NMR $\left(400 \mathrm{MHz}, \mathrm{CDCl}_{3}\right) \delta 6.76(\mathrm{~s}, 1 \mathrm{H}), 6.68$ (s, 1H), $5.99(\mathrm{t}, \mathrm{J}=10.7 \mathrm{~Hz}, 1 \mathrm{H}), 5.88(\mathrm{~s}, 1 \mathrm{H}), 5.82(\mathrm{~s}, 1 \mathrm{H}), 5.65(\mathrm{~d}, \mathrm{~J}=11.4 \mathrm{~Hz}, 1 \mathrm{H}), 5.47(\mathrm{~s}, 1 \mathrm{H})$, $3.35-3.19(\mathrm{~m}, 4 \mathrm{H}), 3.05(\mathrm{~s}, 1 \mathrm{H}), 1.76-1.68(\mathrm{~m}, 2 \mathrm{H}), 1.67-1.60(\mathrm{~m}, 1 \mathrm{H}), 1.56-1.42(\mathrm{~m}, 3 \mathrm{H}), 1.37-$ $1.28(\mathrm{~m}, 2 \mathrm{H}), 0.90(\mathrm{t}, \mathrm{J}=7.3 \mathrm{~Hz}, 3 \mathrm{H}) .{ }^{13} \mathbf{C}$ NMR $\left(101 \mathrm{MHz}, \mathrm{CDCl}_{3}\right) \delta 166.2,149.2,145.3,145.2$, $141.3,140.8,121.4,103.3,103.0,100.4,50.2,46.4,44.1,39.2,39.0,36.8,31.7,20.1$, 13.7. ESIHR calcd for $\mathrm{C}_{19} \mathrm{H}_{24} \mathrm{NO}_{3}{ }^{+}\left([\mathrm{M}+\mathrm{H}]^{+}\right)$314.1751, found 314.1746. IR $v\left(\mathrm{~cm}^{-1}\right) 3294,3006,2961,2871$, 1654, 1625, 751. M. P. $94^{\circ}$ C. EA calcd for C, 72.82; H, 7.40; N, 4.47; found C, 72.91; H, 7.67; N, 4.47. $[\alpha]^{25} \mathrm{D}=+60.8\left(c=1.080, \mathrm{CHCl}_{3}\right)$ for a $97 \%$ ee sample. The enantiomeric purity of this compound was determined by HPLC analysis in comparison with authentic racemic material (Chiralpak OD-H, 92:8 hexanes:i-PrOH, $0.8 \mathrm{~mL} / \mathrm{min}, 254 \mathrm{~nm}$ ): $t_{\text {major }}=17.786 \mathrm{~min}, t_{\text {minor }}=15.923$ $\min$.

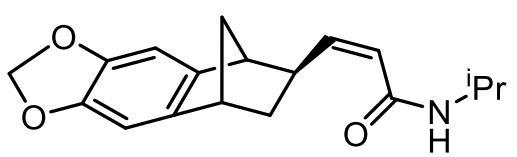

3he: Following the general procedure, $\mathbf{1 h}(11.3 \mathrm{mg}, 0.100 \mathrm{mmol})$ and $\mathbf{2 e}(37.2 \mathrm{mg}, 0.200 \mathrm{mmol})$ were converted to the chiral hydroalkenylation product. Purification by silica gel chromatography gave $21.1 \mathrm{mg}$ (71\% yield) of $\mathbf{3 h e}$ as a white solid. ${ }^{1} \mathbf{H} \mathbf{N M R}\left(400 \mathrm{MHz}, \mathrm{CDCl}_{3}\right) \delta 6.76(\mathrm{~s}, 1 \mathrm{H}), 6.68$ (s, 1H), 6.04-5.94 (m, 1H), $5.88(\mathrm{~d}, \mathrm{~J}=1.3 \mathrm{~Hz}, 1 \mathrm{H}), 5.82(\mathrm{~d}, \mathrm{~J}=1.3 \mathrm{~Hz}, 1 \mathrm{H}), 5.62(\mathrm{dd}, \mathrm{J}=11.4,0.7$ $\mathrm{Hz}, 1 \mathrm{H}), 5.26(\mathrm{~s}, 1 \mathrm{H}), 4.31-3.86(\mathrm{~m}, 1 \mathrm{H}), 3.30(\mathrm{dd}, \mathrm{J}=13.6,3.7 \mathrm{~Hz}, 2 \mathrm{H}), 3.06(\mathrm{~s}, 1 \mathrm{H}), 1.76-1.68$ $(\mathrm{m}, 2 \mathrm{H}), 1.69-1.61(\mathrm{~m}, 1 \mathrm{H}), 1.53(\mathrm{dt}, \mathrm{J}=11.9,4.0 \mathrm{~Hz}, 1 \mathrm{H}), 1.14(\mathrm{dd}, \mathrm{J}=8.0,6.7 \mathrm{~Hz}, 6 \mathrm{H}) .{ }^{13} \mathbf{C ~ N M R}$ 
$\left(101 \mathrm{MHz}, \mathrm{CDCl}_{3}\right) \delta 165.4,149.2,145.3,145.2,141.4,140.9,121.6,103.3,103.0,100.4,50.2,46.4$, 44.2, 41.1, 39.2, 36.8, 22.9, 22.8. ESI-HR calcd for $\mathrm{C}_{18} \mathrm{H}_{22} \mathrm{NO}_{3}{ }^{+}\left([\mathrm{M}+\mathrm{H}]^{+}\right) 300.1594$, found 300.1587. IR $v\left(\mathrm{~cm}^{-1}\right) 3286,3005,2968,2871,1653,1622,751$. M. P. $170{ }^{\circ} \mathrm{C}$. EA calcd for C, 72.22; H, 7.07; N, 4.68; found C, 72.33; H, 7.32; N, 4.51. $[\alpha]^{25} \mathrm{D}=+65.6\left(c=1.045, \mathrm{CHCl}_{3}\right)$ for a 98\% ee sample. The enantiomeric purity of this compound was determined by HPLC analysis in comparison with authentic racemic material (Chiralpak OD-H, 95:5 hexanes:i-PrOH, $0.8 \mathrm{~mL} / \mathrm{min}$, $254 \mathrm{~nm}$ ): $t_{\text {major }}=19.235 \mathrm{~min}, t_{\text {minor }}=17.934 \mathrm{~min}$.<smiles>CC1CC2CC1C[C@@H]2/C=C\C(=O)Nc1ccc(Br)cc1</smiles>

3ie: Following the general procedure, $\mathbf{1 i}(22.6 \mathrm{mg}, 0.100 \mathrm{mmol})$ and $\mathbf{2 e}(37.2 \mathrm{mg}, 0.200 \mathrm{mmol})$ were converted to the chiral hydroalkenylation product. Purification by silica gel chromatography gave $38.2 \mathrm{mg}$ (93\% yield) of 3ie as a white solid. ${ }^{1} \mathbf{H}$ NMR (400 MHz, $\left.\mathrm{CDCl}_{3}\right) \delta 7.46-7.35$ (m, 4H), 6.75 (s, 1H), $6.69(\mathrm{~s}, 1 \mathrm{H}), 6.24-6.13(\mathrm{~m}, 1 \mathrm{H}), 5.86(\mathrm{dd}, \mathrm{J}=23.7,1.3 \mathrm{~Hz}, 2 \mathrm{H}), 5.77(\mathrm{~d}, \mathrm{~J}=11.3 \mathrm{~Hz}, 1 \mathrm{H})$, 3.45-3.34 (m, 1H), $3.30(\mathrm{~d}, \mathrm{~J}=1.8 \mathrm{~Hz}, 1 \mathrm{H}), 3.09(\mathrm{~s}, 1 \mathrm{H}), 1.74(\mathrm{~s}, 2 \mathrm{H}), 1.56(\mathrm{dt}, \mathrm{J}=11.9,4.0 \mathrm{~Hz}$, 1H), 1.35-1.19 (m, 2H). ${ }^{13} \mathrm{C}$ NMR (101 MHz, $\left.\mathrm{CDCl}_{3}\right) \delta 164.1,152.7,145.4,145.3,141.3,140.6$, 137.0, 131.9, 121.2, 120.8, 116.7, 103.3, 103.1, 100.4, 50.1, 46.4, 44.1, 39.4, 36.8. ESI-HR calcd for $\mathrm{C}_{21} \mathrm{H}_{18} \mathrm{BrNNaO}_{3}{ }^{+}\left([\mathrm{M}+\mathrm{Na}]^{+}\right)$434.0362, found 434.0353. IR $v\left(\mathrm{~cm}^{-1}\right)$ 3297, 3010, 2966, 2871, $1660,1628,1527,752$. M. P. $96^{\circ} \mathrm{C} .[\alpha]^{25} \mathrm{D}=+51.9\left(c=1.555, \mathrm{CHCl}_{3}\right)$ for a $79 \%$ ee sample. The enantiomeric purity of this compound was determined by HPLC analysis in comparison with authentic racemic material (Chiralpak OD-H, 92:8 hexanes: $i-\mathrm{PrOH}, 0.8 \mathrm{~mL} / \mathrm{min}, 254 \mathrm{~nm}$ ): $t_{\text {major }}=$ $25.243 \mathrm{~min}, t_{\mathrm{minor}}=21.296 \mathrm{~min}$.

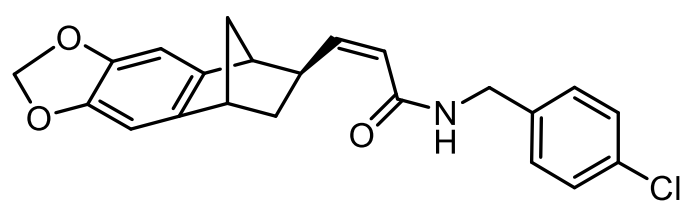

3je: Following the general procedure, $\mathbf{1 j}(19.5 \mathrm{mg}, 0.100 \mathrm{mmol})$ and $\mathbf{2 e}(37.2 \mathrm{mg}, 0.200 \mathrm{mmol})$ were converted to the chiral hydroalkenylation product. Purification by silica gel chromatography gave $30.3 \mathrm{mg}$ (63\% yield) of 3je as a white solid. ${ }^{1} \mathbf{H}$ NMR (400 MHz, $\left.\mathrm{CDCl}_{3}\right) \delta 7.29-7.26(\mathrm{~m}, 2 \mathrm{H}), 7.19$ $(\mathrm{d}, \mathrm{J}=8.5 \mathrm{~Hz}, 2 \mathrm{H}), 6.74(\mathrm{~s}, 1 \mathrm{H}), 6.69(\mathrm{~s}, 1 \mathrm{H}), 6.06(\mathrm{dd}, \mathrm{J}=11.2,10.2 \mathrm{~Hz}, 1 \mathrm{H}), 5.87(\mathrm{dd}, \mathrm{J}=21.1$, 
$1.4 \mathrm{~Hz}, 2 \mathrm{H}), 5.74(\mathrm{~s}, 1 \mathrm{H}), 5.68(\mathrm{dd}, \mathrm{J}=11.4,0.9 \mathrm{~Hz}, 1 \mathrm{H}), 4.49-4.32(\mathrm{~m}, 2 \mathrm{H}), 3.38-3.22(\mathrm{~m}, 2 \mathrm{H})$, $3.06(\mathrm{~s}, 1 \mathrm{H}), 1.72(\mathrm{~d}, \mathrm{~J}=9.6 \mathrm{~Hz}, 2 \mathrm{H}), 1.67-1.60(\mathrm{~m}, 1 \mathrm{H}), 1.53(\mathrm{dt}, \mathrm{J}=11.9,4.0 \mathrm{~Hz}, 1 \mathrm{H}) .{ }^{13} \mathbf{C} \mathbf{N M R}$ $\left(101 \mathrm{MHz}, \mathrm{CDCl}_{3}\right) \delta 166.0,150.4,145.3,145.2,141.3,140.7,136.9,133.3,129.1,128.8,120.8$, 103.3, 103.1, 100.4, 50.2, 46.4, 44.1, 42.6, 39.4, 36.7. ESI-HR calcd for $\mathrm{C}_{22} \mathrm{H}_{21} \mathrm{ClNO}_{3}{ }^{+}\left([\mathrm{M}+\mathrm{H}]^{+}\right)$ 382.1204, found 382.1192. IR $v\left(\mathrm{~cm}^{-1}\right) 3293,3006,2965,2870,1654,1627,1539,751$. M.P. $113^{\circ} \mathrm{C}$. $[\alpha]^{25} \mathrm{D}=+60.0\left(c=0.940, \mathrm{CHCl}_{3}\right)$ for an $88 \%$ ee sample. The enantiomeric purity of this compound was determined by HPLC analysis in comparison with authentic racemic material (Chiralpak ODH, 92:8 hexanes: $i-\mathrm{PrOH}, 0.8 \mathrm{~mL} / \mathrm{min}, 254 \mathrm{~nm}$ ): $t_{\text {major }}=44.110 \mathrm{~min}, t_{\text {minor }}=35.846 \mathrm{~min}$.

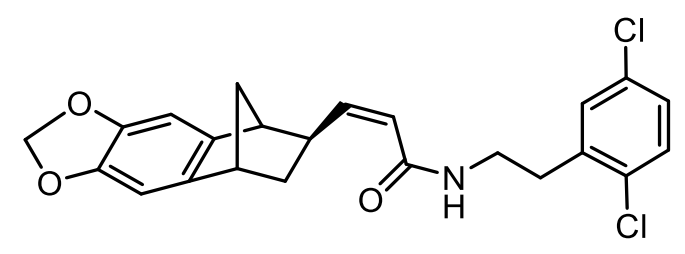

3ke: Following the general procedure, 1k (24.4 mg, $0.100 \mathrm{mmol})$ and $\mathbf{2 e}(37.2 \mathrm{mg}, 0.200 \mathrm{mmol})$ were converted to the chiral hydroalkenylation product. Purification by silica gel chromatography gave $35.6 \mathrm{mg}$ (83\% yield) of 3ke as a white solid. ${ }^{1} \mathbf{H}$ NMR $\left(400 \mathrm{MHz}, \mathrm{CDCl}_{3}\right) \delta$ 7.27-7.24 (m, 1H), $7.20(\mathrm{~d}, \mathrm{~J}=2.5 \mathrm{~Hz}, 1 \mathrm{H}), 7.13(\mathrm{dd}, \mathrm{J}=8.5,2.5 \mathrm{~Hz}, 1 \mathrm{H}), 6.76(\mathrm{~s}, 1 \mathrm{H}), 6.69(\mathrm{~s}, 1 \mathrm{H}), 6.03(\mathrm{dd}, \mathrm{J}$ $=11.2,10.2 \mathrm{~Hz}, 1 \mathrm{H}), 5.86(\mathrm{dd}, \mathrm{J}=21.0,1.4 \mathrm{~Hz}, 2 \mathrm{H}), 5.62(\mathrm{dd}, \mathrm{J}=11.4,0.8 \mathrm{~Hz}, 1 \mathrm{H}), 5.55(\mathrm{~s}, 1 \mathrm{H})$, $3.52(\mathrm{dd}, \mathrm{J}=13.2,6.8 \mathrm{~Hz}, 2 \mathrm{H}), 3.32-3.21(\mathrm{~m}, 2 \mathrm{H}), 3.05(\mathrm{~s}, 1 \mathrm{H}), 2.92(\mathrm{t}, \mathrm{J}=7.0 \mathrm{~Hz}, 2 \mathrm{H}), 1.77-1.69$ $(\mathrm{m}, 2 \mathrm{H}), 1.64(\mathrm{t}, \mathrm{J}=10.2 \mathrm{~Hz}, 1 \mathrm{H}), 1.53(\mathrm{dt}, \mathrm{J}=12.0,4.0 \mathrm{~Hz}, 1 \mathrm{H}) .{ }^{13} \mathbf{C} \mathbf{N M R}\left(101 \mathrm{MHz}, \mathrm{CDCl}_{3}\right) \delta$ $166.3,150.1,145.3,145.2,141.3,140.8,138.4,132.6,132.3,130.8,130.6,128.0,120.9,103.3$, 103.0, 100.4, 50.2, 46.4, 44.1, 39.3, 38.7, 36.7, 33.3. ESI-HR calcd for $\mathrm{C}_{23} \mathrm{H}_{22} \mathrm{Cl}_{2} \mathrm{NO}_{3}{ }^{+}\left([\mathrm{M}+\mathrm{H}]^{+}\right)$ 430.0971, found 430.0958. IR $v\left(\mathrm{~cm}^{-1}\right) 3292,3006,2964,2870,1653,1629,1540,751$. M.P. $86{ }^{\circ} \mathrm{C}$. $[\alpha]^{25} \mathrm{D}=44.7\left(c=1.625, \mathrm{CHCl}_{3}\right)$ for a $97 \%$ ee sample. The enantiomeric purity of this compound was determined by HPLC analysis in comparison with authentic racemic material (Chiralpak ODH, 92:8 hexanes: $i-\mathrm{PrOH}, 0.8 \mathrm{~mL} / \mathrm{min}, 254 \mathrm{~nm}$ ): $t_{\text {major }}=25.623 \mathrm{~min}, t_{\text {minor }}=22.518 \mathrm{~min}$.

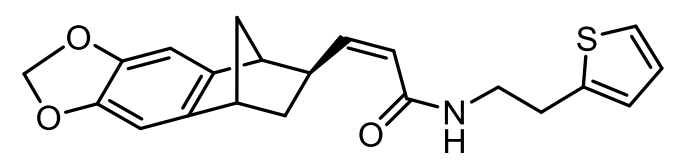

3le: Following the general procedure, $\mathbf{1 l}(18.1 \mathrm{mg}, 0.100 \mathrm{mmol})$ and $\mathbf{2 e}(37.2 \mathrm{mg}, 0.200 \mathrm{mmol})$ were converted to the chiral hydroalkenylation product. Purification by silica gel chromatography gave 
$28.4 \mathrm{mg}$ (77\% yield) of 3le as a light yellow solid. ${ }^{1} \mathbf{H}$ NMR $\left(400 \mathrm{MHz}, \mathrm{CDCl}_{3}\right) \delta 7.13(\mathrm{dd}, \mathrm{J}=5.1$, $0.9 \mathrm{~Hz}, 1 \mathrm{H}), 6.90(\mathrm{dd}, \mathrm{J}=5.1,3.5 \mathrm{~Hz}, 1 \mathrm{H}), 6.78(\mathrm{~d}, \mathrm{~J}=2.8 \mathrm{~Hz}, 1 \mathrm{H}), 6.76(\mathrm{~s}, 1 \mathrm{H}), 6.69(\mathrm{~s}, 1 \mathrm{H}), 6.00$ $(\mathrm{dd}, \mathrm{J}=11.1,10.3 \mathrm{~Hz}, 1 \mathrm{H}), 5.89(\mathrm{~d}, \mathrm{~J}=1.3 \mathrm{~Hz}, 1 \mathrm{H}), 5.83(\mathrm{~d}, \mathrm{~J}=1.2 \mathrm{~Hz}, 1 \mathrm{H}), 5.63(\mathrm{~d}, \mathrm{~J}=11.4 \mathrm{~Hz}$, 1H), $5.58(\mathrm{~s}, 1 \mathrm{H}), 3.63-3.46(\mathrm{~m}, 2 \mathrm{H}), 3.30-3.20(\mathrm{~m}, 2 \mathrm{H}), 3.03(\mathrm{dd}, \mathrm{J}=12.4,5.8 \mathrm{~Hz}, 3 \mathrm{H}), 1.72(\mathrm{~s}$, 2H), 1.63-1.57 (m, 1H), $1.51(\mathrm{dt}, \mathrm{J}=11.9,4.0 \mathrm{~Hz}, 1 \mathrm{H}) .{ }^{13} \mathrm{C}$ NMR $\left(101 \mathrm{MHz}, \mathrm{CDCl}_{3}\right) \delta 166.2$, 149.5, 145.3, 145.2, 141.3 (2C), 140.8, 127.0, 125.3, 123.9, 121.2, 103.3, 103.1, 100.4, 50.1, 46.4, 44.1, 40.6, 39.3, 36.7, 29.9. ESI-HR calcd for $\mathrm{C}_{21} \mathrm{H}_{22} \mathrm{NO}_{3} \mathrm{~S}^{+}\left([\mathrm{M}+\mathrm{H}]^{+}\right) 368.1315$, found 368.1304 . IR $v\left(\mathrm{~cm}^{-1}\right) 3293,3005,2963,2869,1654,1628,1535,751$. M. P. $139{ }^{\circ} \mathrm{C}$. EA calcd for C, 68.64; $\mathrm{H}, 5.76 ; \mathrm{N}, 3.81$; found $\mathrm{C}, 68.43 ; \mathrm{H}, 5.66 ; \mathrm{N}, 3.95 .[\alpha]^{25} \mathrm{D}=+39.8\left(c=1.425, \mathrm{CHCl}_{3}\right)$ for a $97 \%$ ee sample. The enantiomeric purity of this compound was determined by HPLC analysis in comparison with authentic racemic material (Chiralpak OD-H, 92:8 hexanes:i-PrOH, $0.8 \mathrm{~mL} / \mathrm{min}, 254 \mathrm{~nm}$ ): $t_{\text {major }}=36.206 \mathrm{~min}, t_{\text {minor }}=30.878 \mathrm{~min}$.

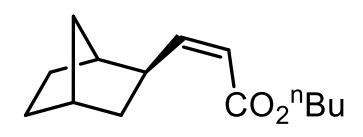

5ab: Following the general procedure, $\mathbf{4 a}(12.8 \mathrm{mg}, 0.100 \mathrm{mmol})$ and $\mathbf{2 b}(94.1 \mathrm{mg}, 0.500 \mathrm{mmol})$ were converted to the chiral hydroalkenylation product. Purification by silica gel chromatography gave $12.7 \mathrm{mg}$ (57\% yield) of $\mathbf{5 a b}$ as a colorless oil. ${ }^{1} \mathbf{H} \mathbf{~ N M R}\left(400 \mathrm{MHz}, \mathrm{CDCl}_{3}\right) \delta 6.06(\mathrm{dd}, \mathrm{J}=$ 11.4, $9.9 \mathrm{~Hz}, 1 \mathrm{H}), 5.60(\mathrm{dd}, \mathrm{J}=11.5,1.0 \mathrm{~Hz}, 1 \mathrm{H}), 4.10(\mathrm{t}, \mathrm{J}=6.7 \mathrm{~Hz}, 2 \mathrm{H}), 3.29(\mathrm{td}, \mathrm{J}=9.3,4.8 \mathrm{~Hz}$, 1H), $2.26(\mathrm{~s}, 1 \mathrm{H}), 2.06(\mathrm{~s}, 1 \mathrm{H}), 1.67$ (m, 3H), 1.54-1.45 (m, 2H), 1.44-1.31 (m, 4H), 1.24-1.15 (m, 3H), $0.94(\mathrm{t}, \mathrm{J}=7.4 \mathrm{~Hz}, 3 \mathrm{H}) .{ }^{13} \mathbf{C}$ NMR $\left(101 \mathrm{MHz}, \mathrm{CDCl}_{3}\right) \delta 166.6,155.6,116.9,63.7,43.0,40.9$, 38.8, 36.5, 36.0, 30.7, 29.6, 28.8, 19.2, 13.7. ESI-HR calcd for $\mathrm{C}_{14} \mathrm{H}_{22} \mathrm{NaO}_{2}{ }^{+}\left([\mathrm{M}+\mathrm{Na}]^{+}\right)$245.1512, found 245.1505. IR $v\left(\mathrm{~cm}^{-1}\right) 3006,2954,2871,1719,1637,750 .[\alpha]^{25} \mathrm{D}=+47.7\left(c=0.635, \mathrm{CHCl}_{3}\right)$ for a $97 \%$ ee sample. The enantiomeric purity of this compound was determined by HPLC analysis in comparison with authentic racemic material (Chiralpak ID, 100:0 hexanes:i-PrOH, $0.3 \mathrm{~mL} / \mathrm{min}$, $210 \mathrm{~nm}): t_{\text {major }}=33.958 \mathrm{~min}, t_{\text {minor }}=36.821 \mathrm{~min}$.

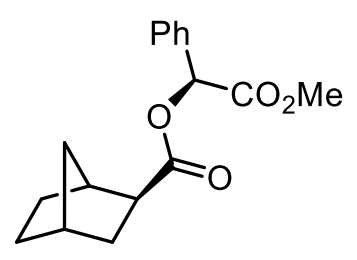


6: Following the literature reported procedure, 6 was obtained in $79.9 \mathrm{mg}(0.277 \mathrm{mmol}, 46 \%$ total yield for 3 steps) as a colorless oil. ${ }^{1} \mathbf{H}$ NMR $\left(400 \mathrm{MHz}, \mathrm{C}_{6} \mathrm{D}_{6}\right) \delta$ 7.48-7.43 (m, 2H), 7.10-7.02 (m, 3H), $6.06(\mathrm{~s}, 1 \mathrm{H}), 3.18(\mathrm{~d}, \mathrm{~J}=5.8 \mathrm{~Hz}, 3 \mathrm{H}), 2.86(\mathrm{~d}, \mathrm{~J}=3.4 \mathrm{~Hz}, 1 \mathrm{H}), 2.33(\mathrm{dd}, \mathrm{J}=8.8,5.0 \mathrm{~Hz}, 1 \mathrm{H})$, 2.10-2.00 (m, 2H), 1.74-1.68 (m, 1H), $1.26(\mathrm{~m}, 3 \mathrm{H}), 1.06(\mathrm{~m}, 1 \mathrm{H}), 1.02-0.90(\mathrm{~m}, 2 \mathrm{H}) .{ }^{13} \mathbf{C}$ NMR $\left(101 \mathrm{MHz}, \mathrm{C}_{6} \mathrm{D}_{6}\right) \delta 175.8,170.1,135.5,129.8,129.5,128.7,75.4,52.5,47.1,42.1,37.3,37.0,34.6$, 30.2, 29.5. ESI-HR calcd for $\mathrm{C}_{17} \mathrm{H}_{20} \mathrm{NaO}_{4}{ }^{+}\left([\mathrm{M}+\mathrm{Na}]^{+}\right) 311.1254$, found 311.1243. IR $v\left(\mathrm{~cm}^{-1}\right) 3006$, $2953,2872,1735,1699,1455,750 .[\alpha]^{25} \mathrm{D}=+96.9\left(c=3.815, \mathrm{CHCl}_{3}\right)$. 


\section{General Information for Computation:}

All calculations were performed with the Gaussian 09 program. (Full citation of Gaussian 09: Frisch, M. J.; Trucks, G. W.; Schlegel, H. B.; Scuseria, G. E.; Robb, M. A.; Cheeseman, J. R.; Scalmani, G.; Barone, V.; Mennucci, B.; Petersson, G. A.; Nakatsuji, H.; Caricato, M.; Li, X.; Hratchian, H. P.; Izmaylov, A. F.; Bloino, J.; Zheng, G.; Sonnenberg, J. L.; Hada, M.; Ehara, M.; Toyota, K.; Fukuda, R.; Hasegawa, J.; Ishida, M.; Nakajima, T.; Honda, Y.; Kitao, O.; Nakai, H.; Vreven, T.; Montgomery, J. A., Jr.; Peralta, J. E.; Ogliaro, F.; Bearpark, M.; Heyd, J. J.; Brothers, E.; Kudin, K. N.; Staroverov, V. N.; Keith, T.; Kobayashi, R.; Normand, J.; Raghavachari, K.; Rendell, A.; Burant, J. C.; Iyengar, S. S.; Tomasi, J.; Cossi, M.; Rega, N.; Millam, J. M.; Klene, M.; Knox, J. E.; Cross, J. B.; Bakken, V.; Adamo, C.; Jaramillo, J.; Gomperts, R.; Stratmann, R. E.; Yazyev, O.; Austin, A. J.; Cammi, R.; Pomelli, C.; Ochterski, J. W.; Martin, R. L.; Morokuma, K.; Zakrzewski, V. G.; Voth, G. A.; Salvador, P.; Dannenberg, J. J.; Dapprich, S.; Daniels, A. D.; Farkas, O.; Foresman, J. B.; Ortiz, J. V.; Cioslowski, J.; Fox, D. J.; Gaussian 09, revision B.01; Gaussian Inc.: Wallingford, CT, 2010.) Density functional theory calculations using the M06 functional ((a) Zhao, Y.; Truhlar, D. G. Theor. Chem. Acc. 2008, 120, 215-241. (b) Zhao, Y.; Truhlar, D. G. Acc. Chem. Res. 2008, 41, 157-167.) Geometry optimizations were conducted with the Gaussian 09 software package, B3LYP functional, and LANL2DZ basis set for Ir and 6-31g(d,p) basis set for all other atoms. Single-point energy calculations were conducted with the M06 functional and SDD basis set for Ir and the 6-311++g(d,p) basis set for all other atoms, along with the SMD DCE solvent correction. Thermal corrections were applied to the optimized geometries to provide Gibbs free energies. 


\section{References}

1 Tsuchikama, K.; Kasagawa, M.; Endo, K.; Shibata, T. Org. Lett. 2009, 11, 1821.

2 Fan, B,; Yang, Q.; Hu, J.; Fan, C.; Li, S.; Yu, L,; Huang, C.; Tsang, W. W.; Kwong, F. Y. Angew. Chem. Int. Ed. 2012, 51, 7821

3 Sun, X.; Zhao, W.; Li, B.-J. Chem. Commun. 2020, 56, 1298.

4 Kumareswaran, R.; Nandi, M.; RajanBabu, T. V. Org. Lett. 2003, 5, 4345. 


\section{Copies of NMR spectra}
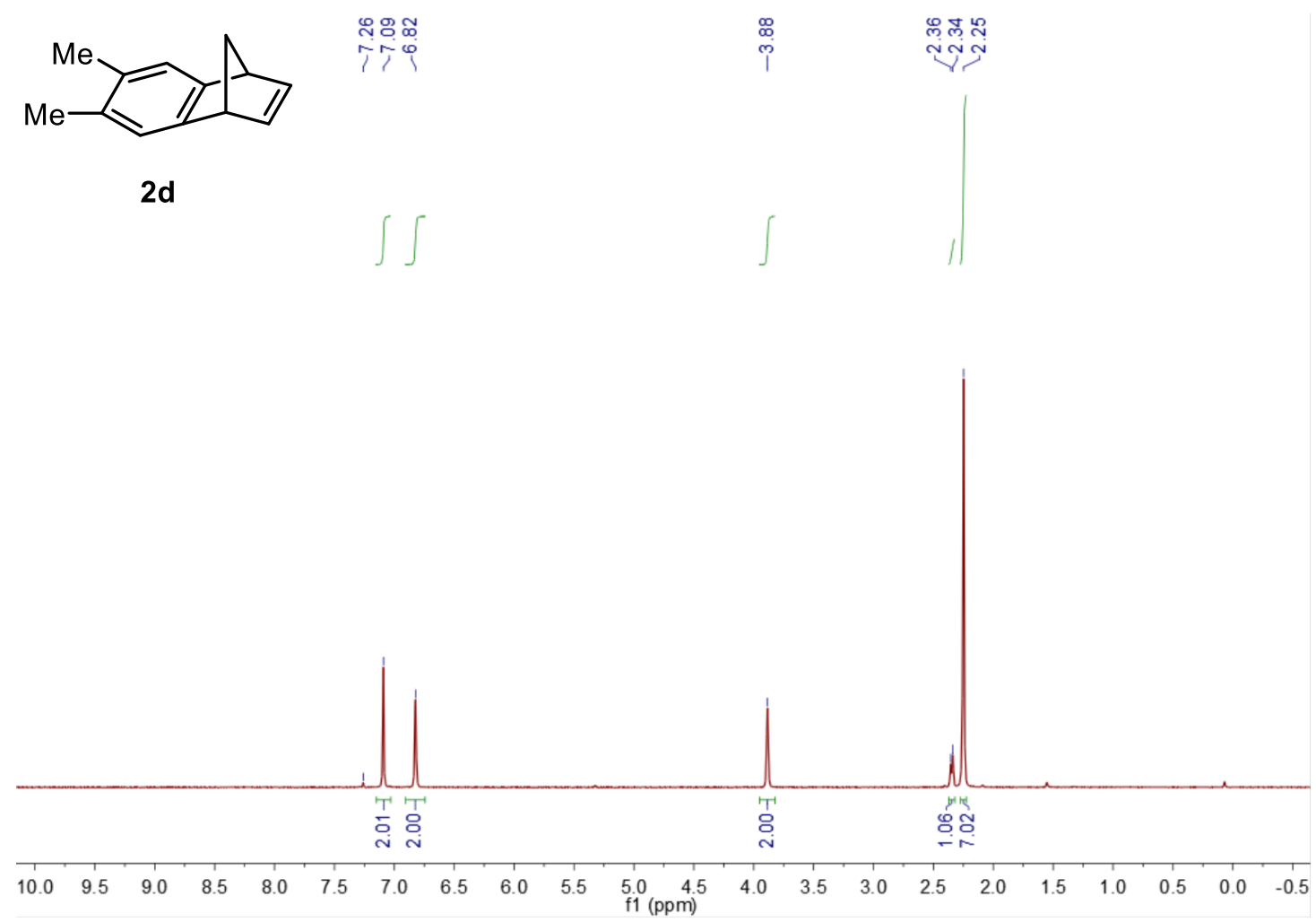

Figure S5. ${ }^{1} \mathrm{H}$ NMR $\left(400 \mathrm{MHz}, \mathrm{CDCl}_{3}\right)$ of $\mathbf{2 d}$.

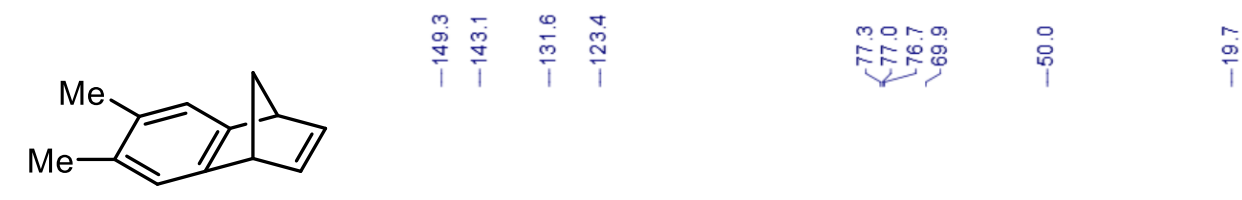

2d

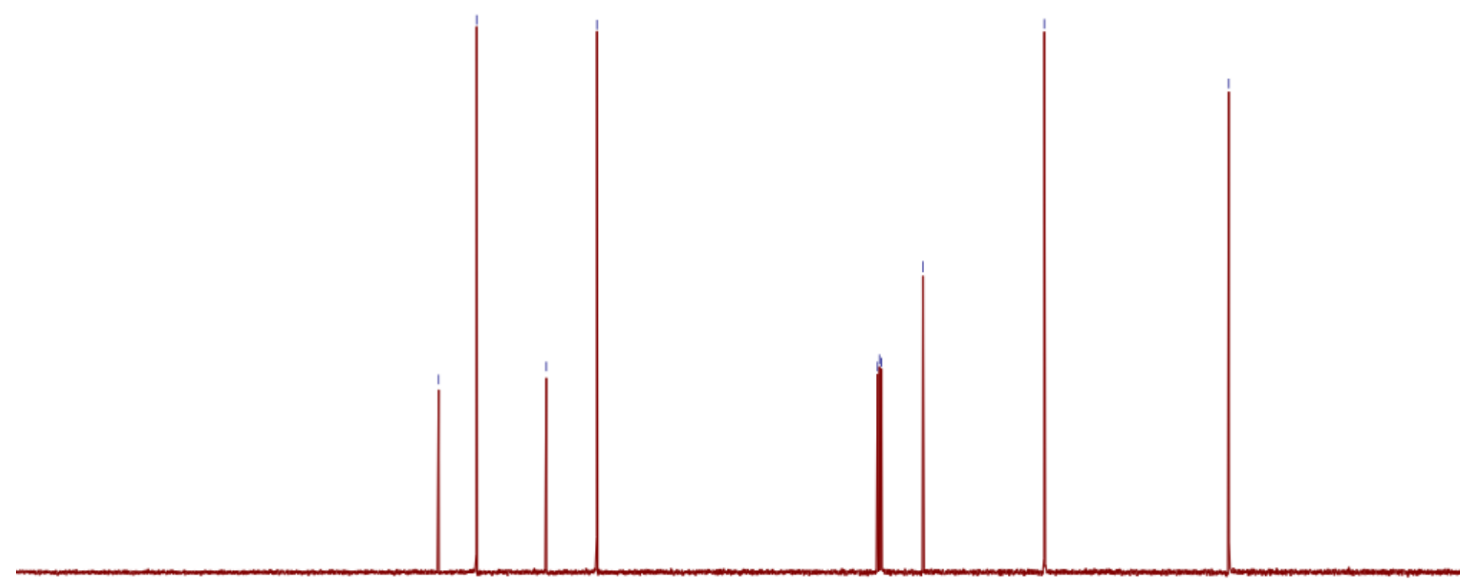

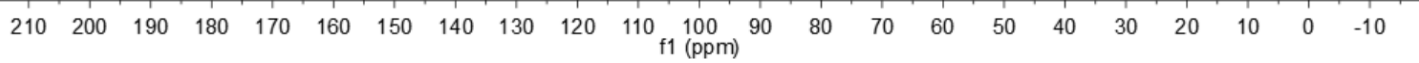

Figure S6. ${ }^{13} \mathrm{C}$ NMR $\left(101 \mathrm{MHz}, \mathrm{CDCl}_{3}\right)$ of $\mathbf{2 d}$. 

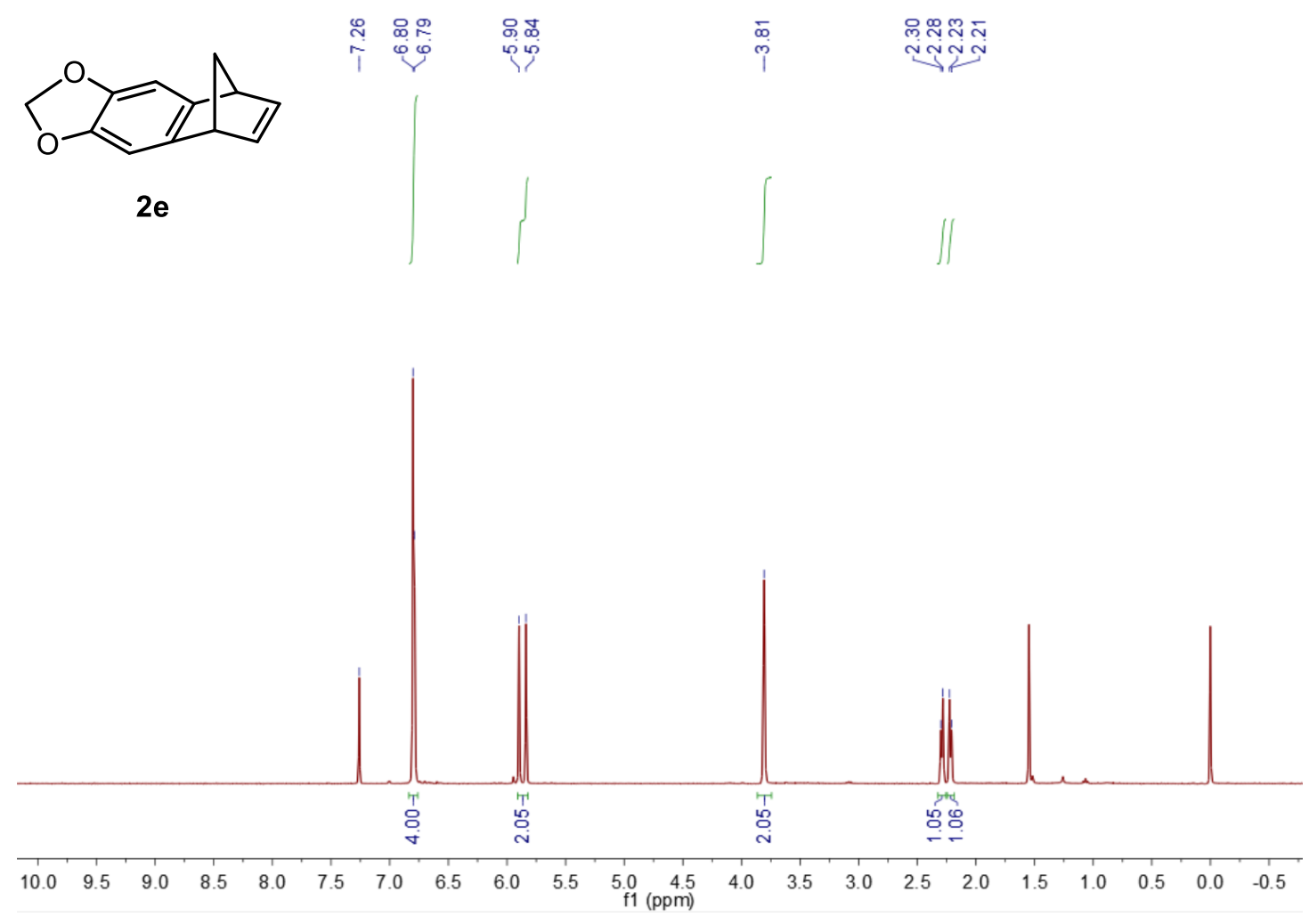

Figure S7. ${ }^{1} \mathrm{H}$ NMR $\left(400 \mathrm{MHz}, \mathrm{CDCl}_{3}\right)$ of $2 \mathbf{e}$.

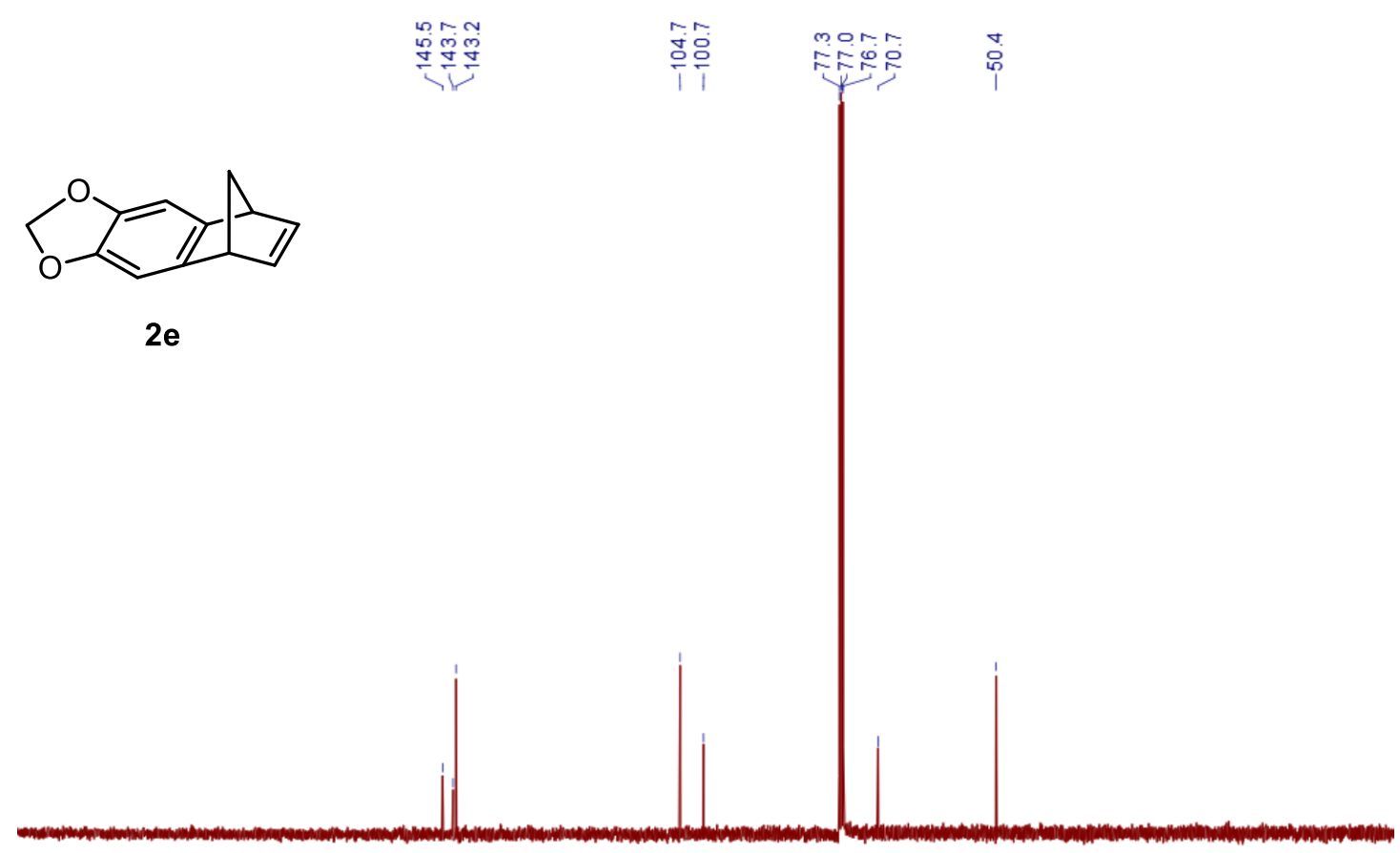

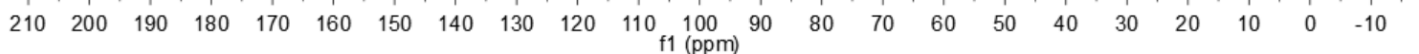

Figure S8. ${ }^{13} \mathrm{C}$ NMR $\left(101 \mathrm{MHz}, \mathrm{CDCl}_{3}\right)$ of $\mathbf{2 e}$. 


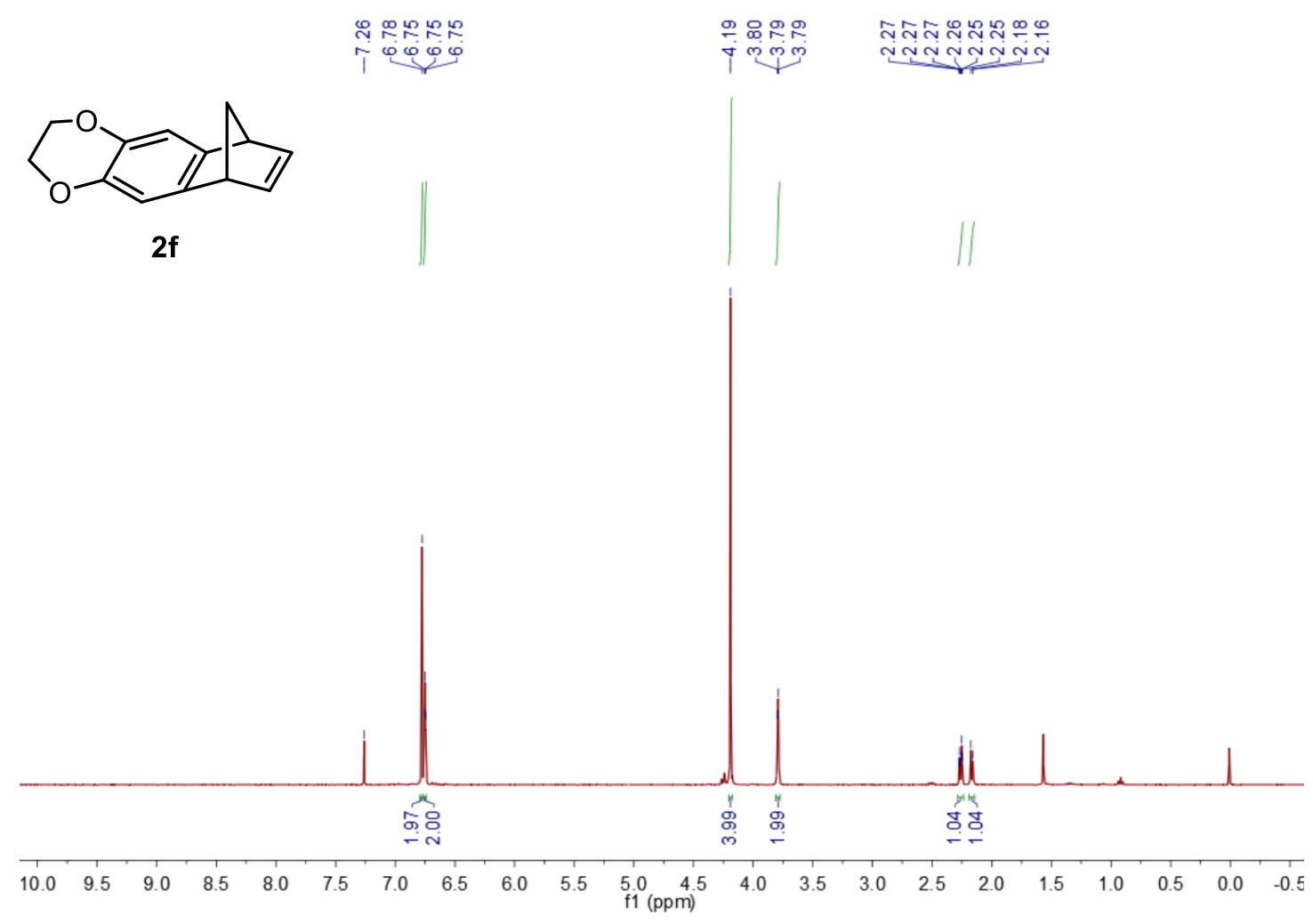

Figure S9. ${ }^{1} \mathrm{H}$ NMR $\left(400 \mathrm{MHz}, \mathrm{CDCl}_{3}\right)$ of $\mathbf{2 f}$.
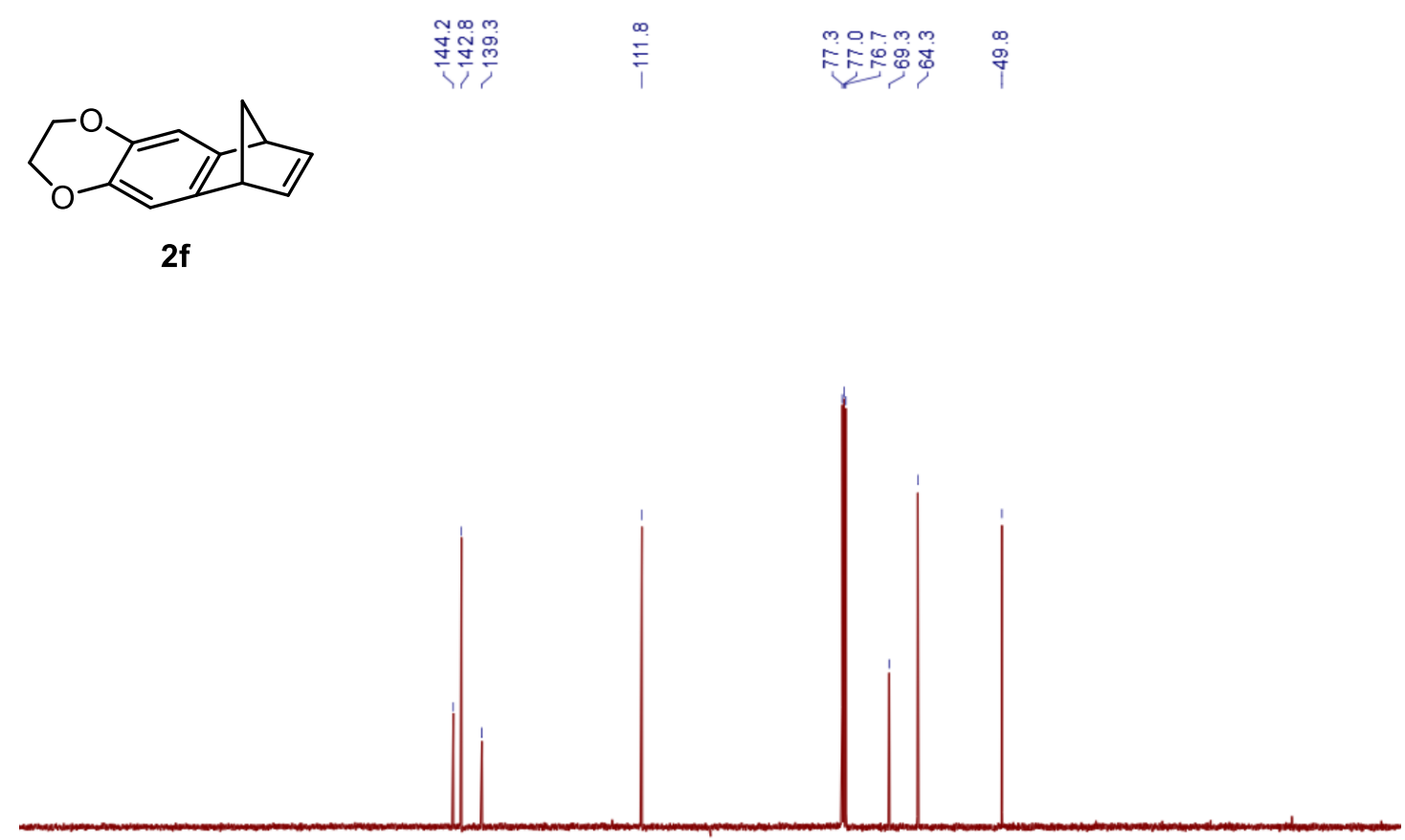

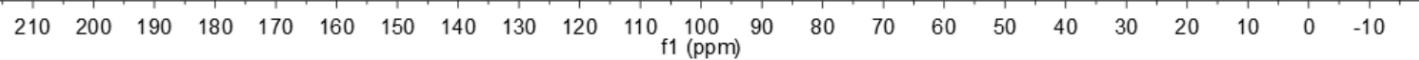

Figure S10. ${ }^{13} \mathrm{C}$ NMR (101 MHz, $\left.\mathrm{CDCl}_{3}\right)$ of $\mathbf{2 f}$. 


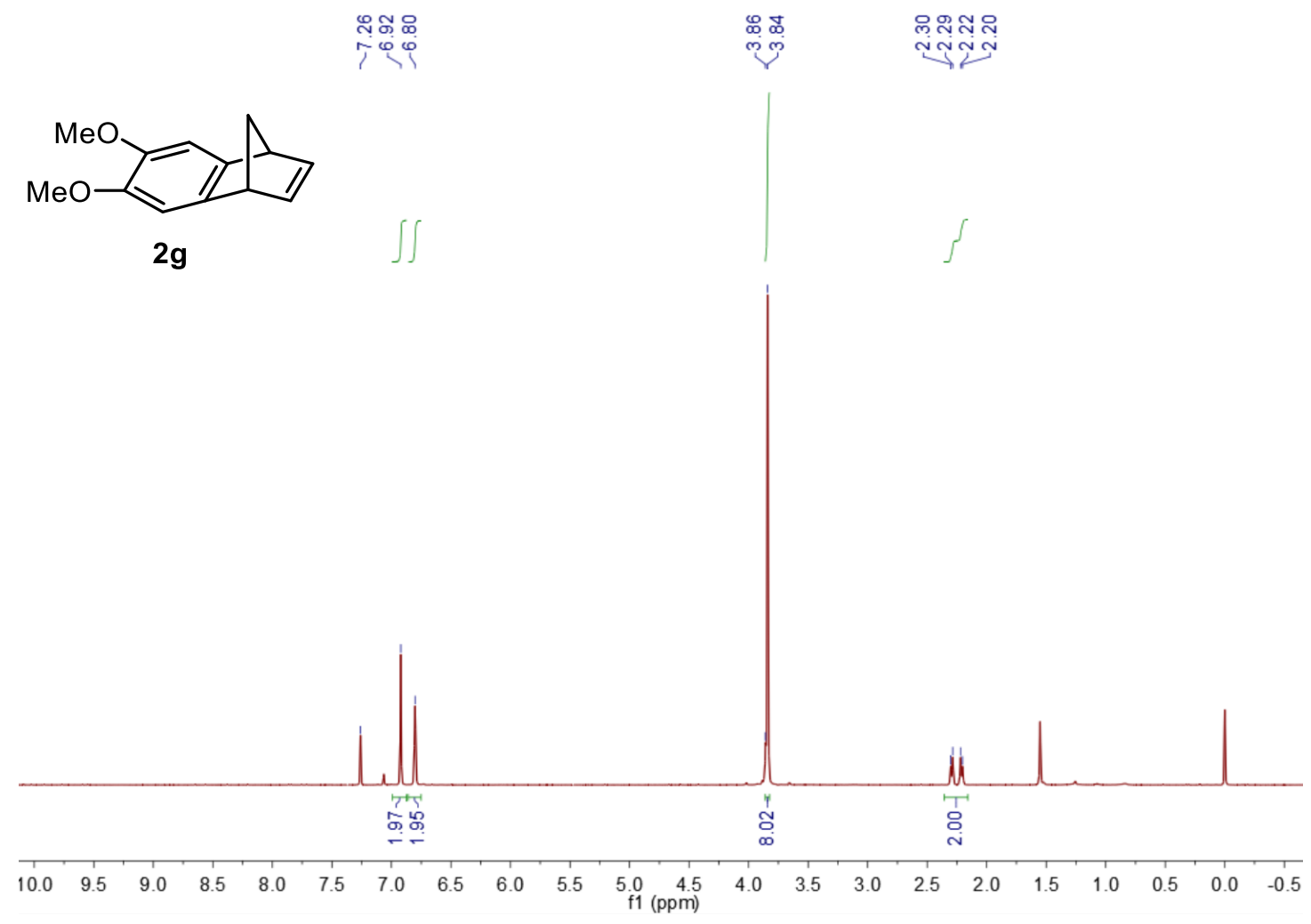

Figure S11. ${ }^{1} \mathrm{H}$ NMR $\left(400 \mathrm{MHz}, \mathrm{CDCl}_{3}\right)$ of $\mathbf{2 g}$.

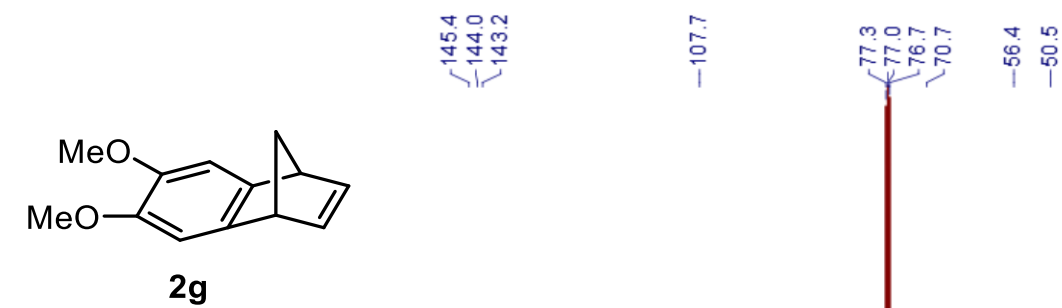

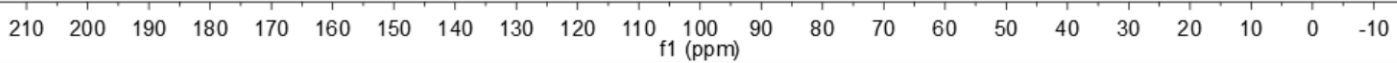

Figure S12. ${ }^{13} \mathrm{C}$ NMR $\left(101 \mathrm{MHz}, \mathrm{CDCl}_{3}\right)$ of $\mathbf{2 g}$. 

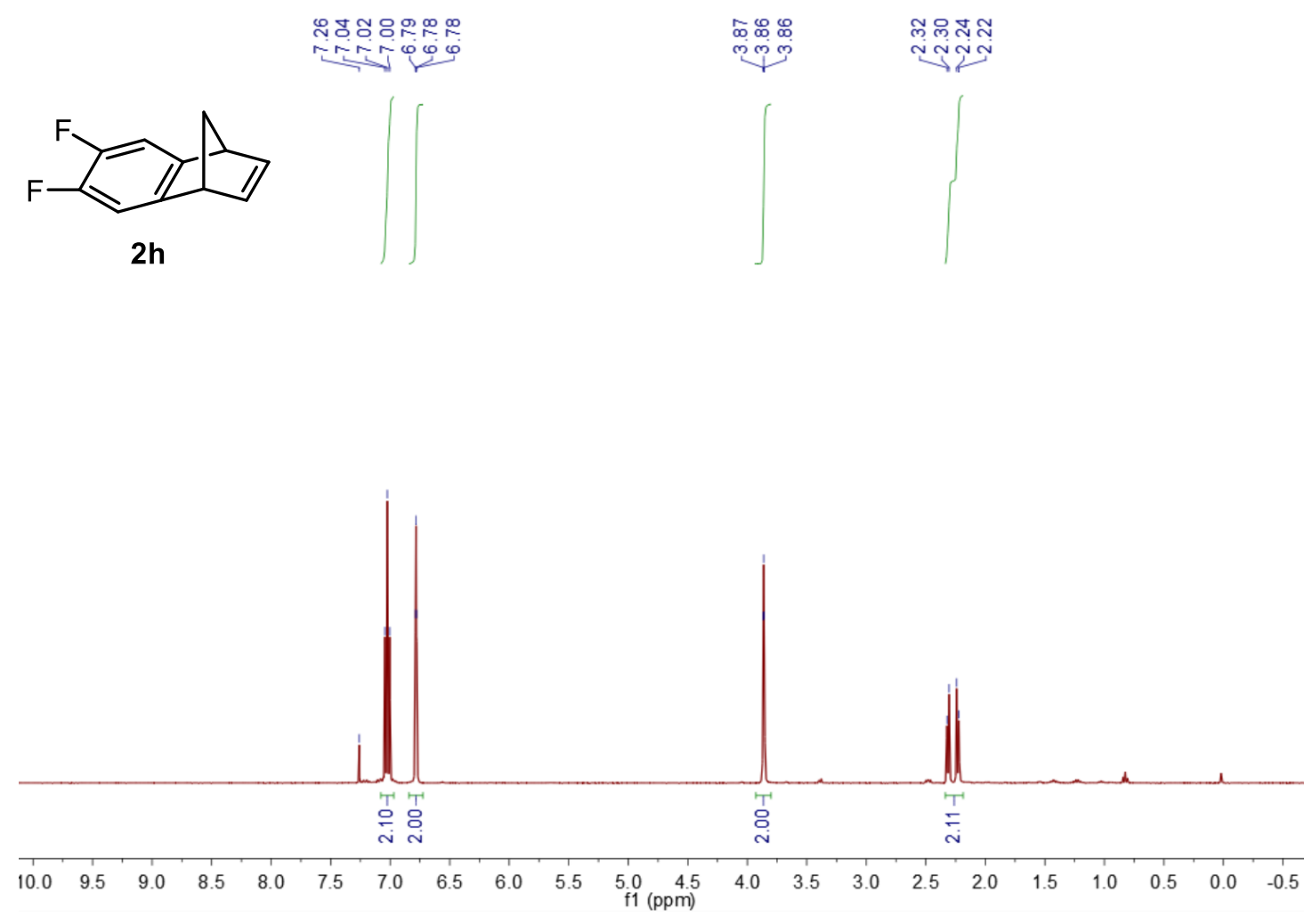

Figure S13. ${ }^{1} \mathrm{H}$ NMR $\left(400 \mathrm{MHz}, \mathrm{CDCl}_{3}\right)$ of $\mathbf{2} \mathbf{h}$.
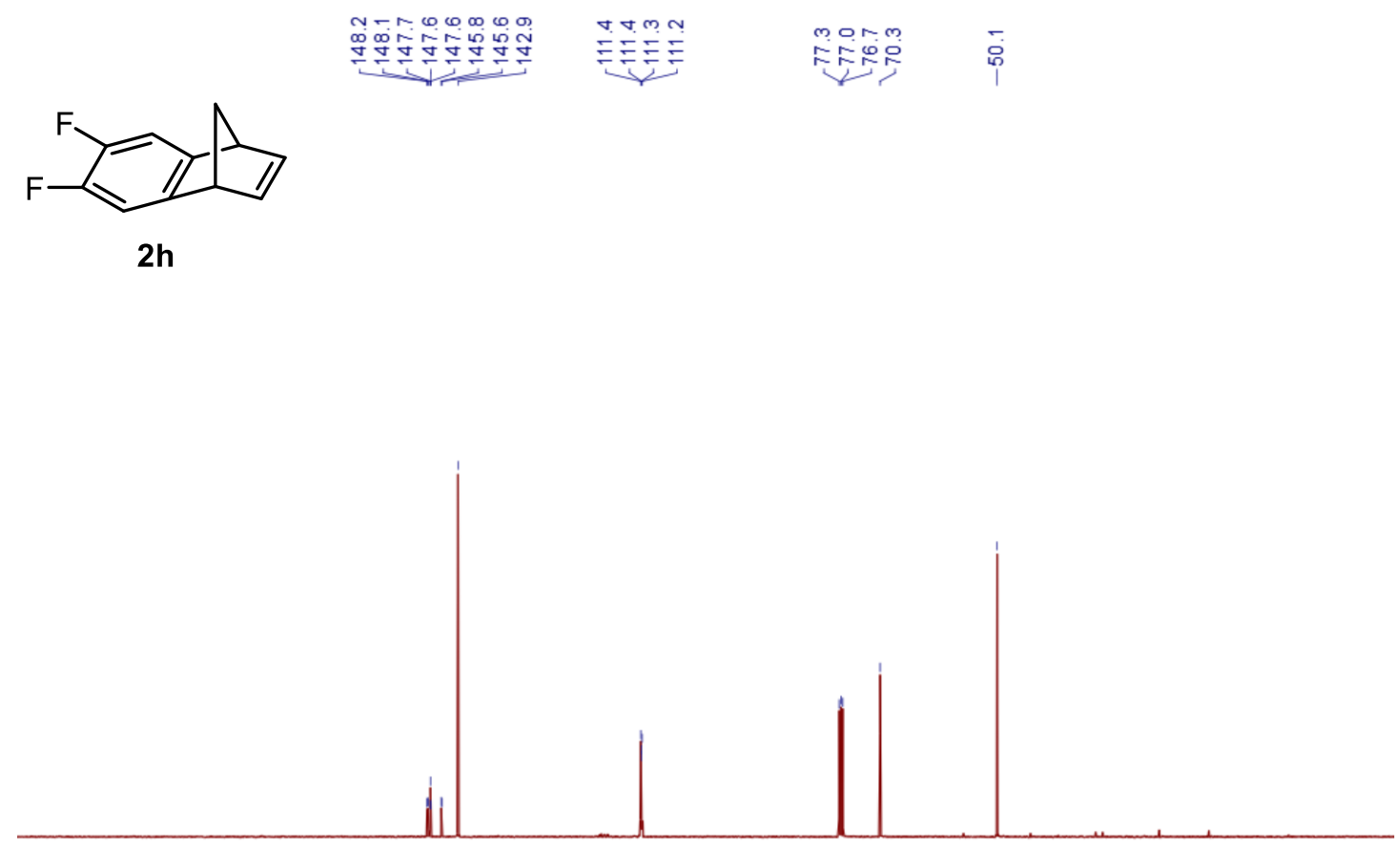

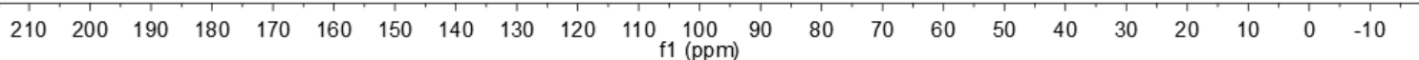

Figure S14. ${ }^{13} \mathrm{C}$ NMR $\left(101 \mathrm{MHz}, \mathrm{CDCl}_{3}\right)$ of $\mathbf{2 h}$. 

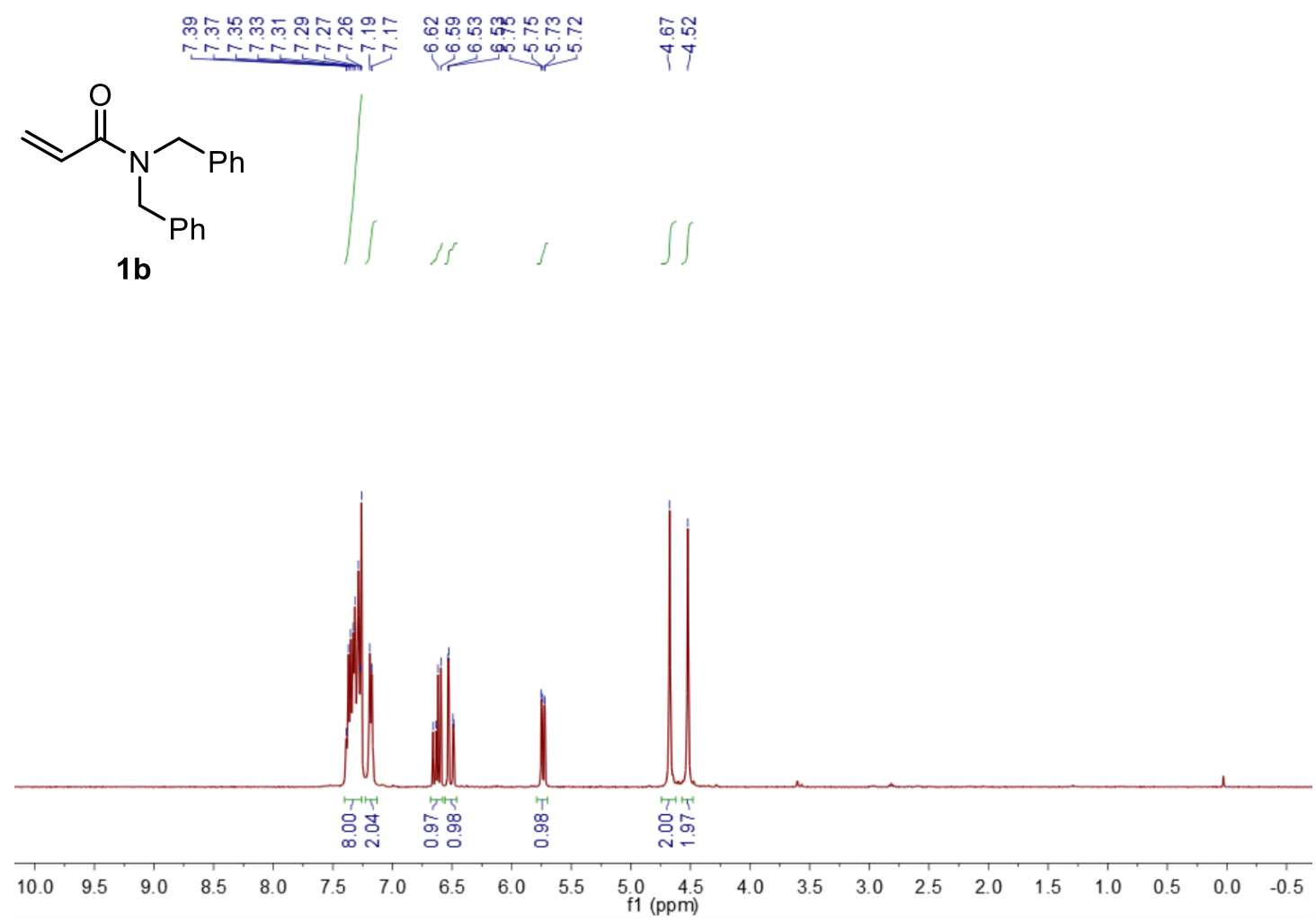

Figure S15. ${ }^{1} \mathrm{H}$ NMR $\left(400 \mathrm{MHz}, \mathrm{CDCl}_{3}\right)$ of $\mathbf{1 b}$.

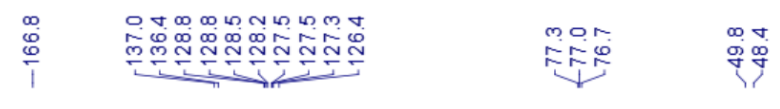<smiles>C=CC(=O)N(Cc1ccccc1)Cc1ccccc1</smiles>

1b

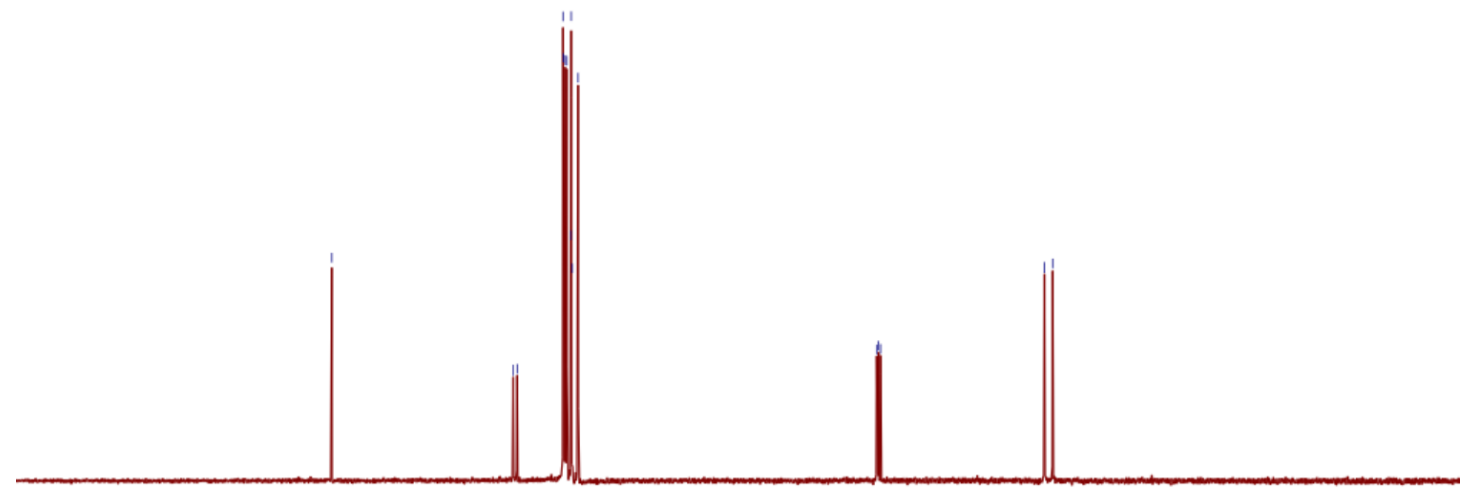

$\begin{array}{llllllllllllllllllllll}210 & 200 & 190 & 180 & 170 & 160 & 150 & 140 & 130 & 120 & 110 \begin{array}{c}100 \\ \mathrm{f} 1(\mathrm{ppm})\end{array} & 90 & 80 & 70 & 60 & 50 & 40 & 30 & 20 & 10 & 0 & -10\end{array}$

Figure S16. ${ }^{13} \mathrm{C}$ NMR (101 MHz, $\left.\mathrm{CDCl}_{3}\right)$ of $\mathbf{1 b}$. 


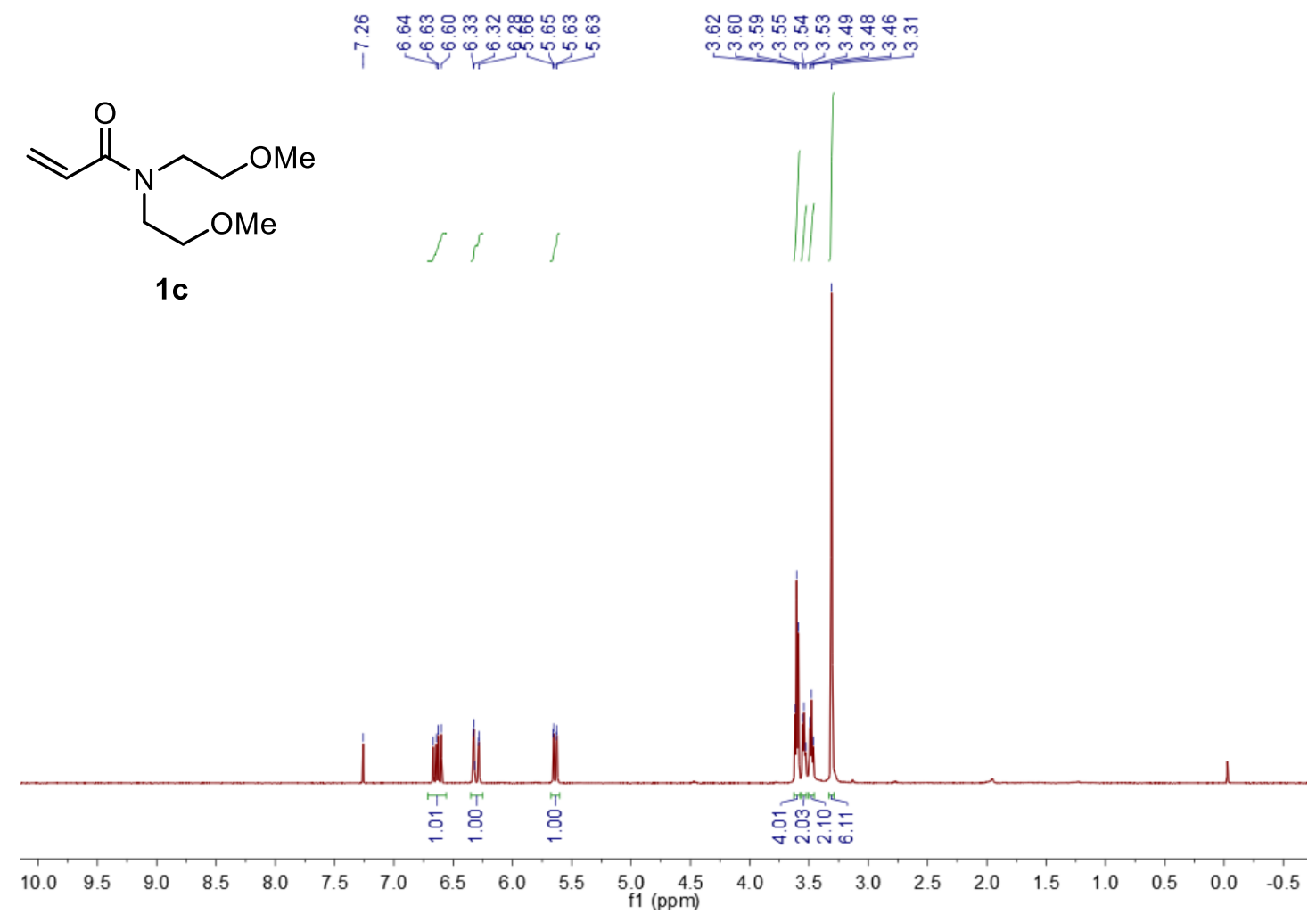

Figure S17. ${ }^{1} \mathrm{H}$ NMR (400 MHz, $\left.\mathrm{CDCl}_{3}\right)$ of $\mathbf{1 c}$.

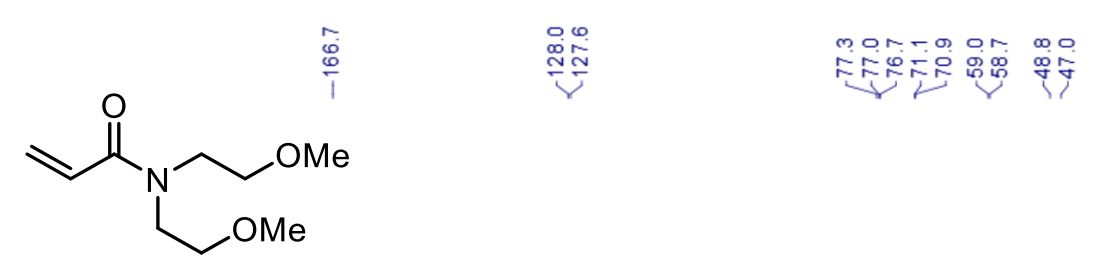

$1 \mathrm{c}$

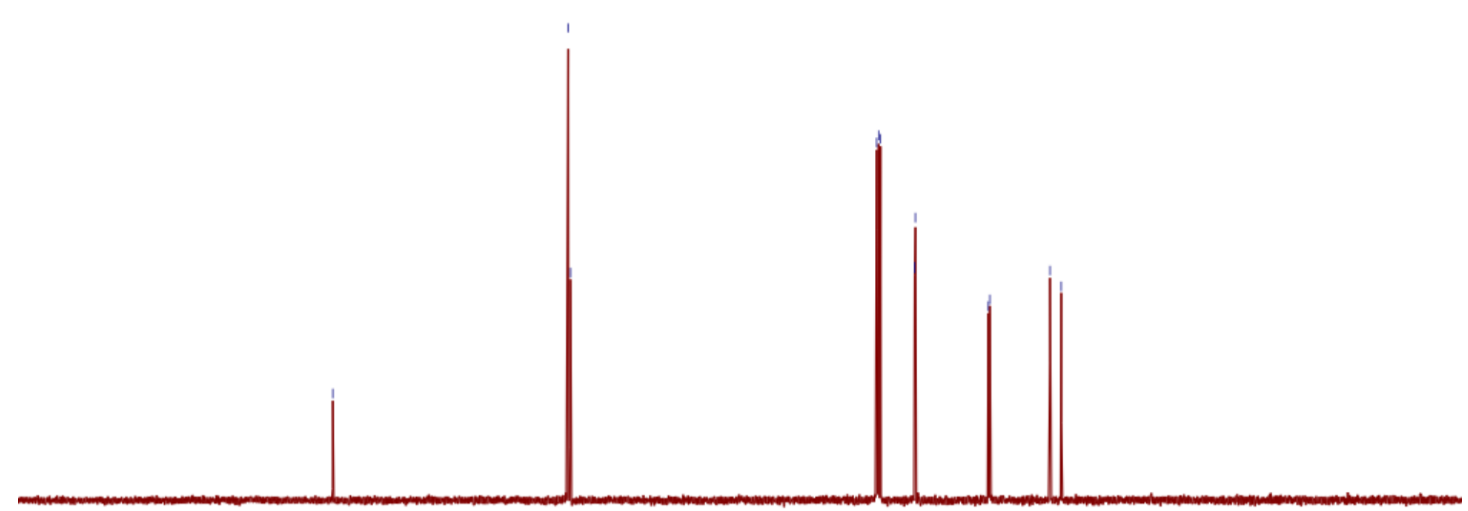

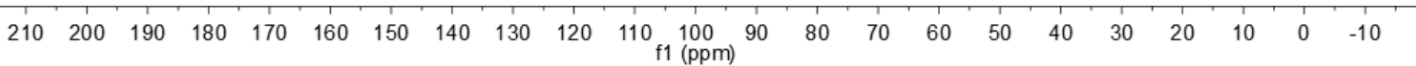

Figure S18. ${ }^{13} \mathrm{C}$ NMR $\left(101 \mathrm{MHz}, \mathrm{CDCl}_{3}\right)$ of $\mathbf{1 c}$. 
<smiles>C=CC(=O)N1CCCCC1</smiles>

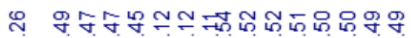

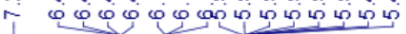

$1 d$
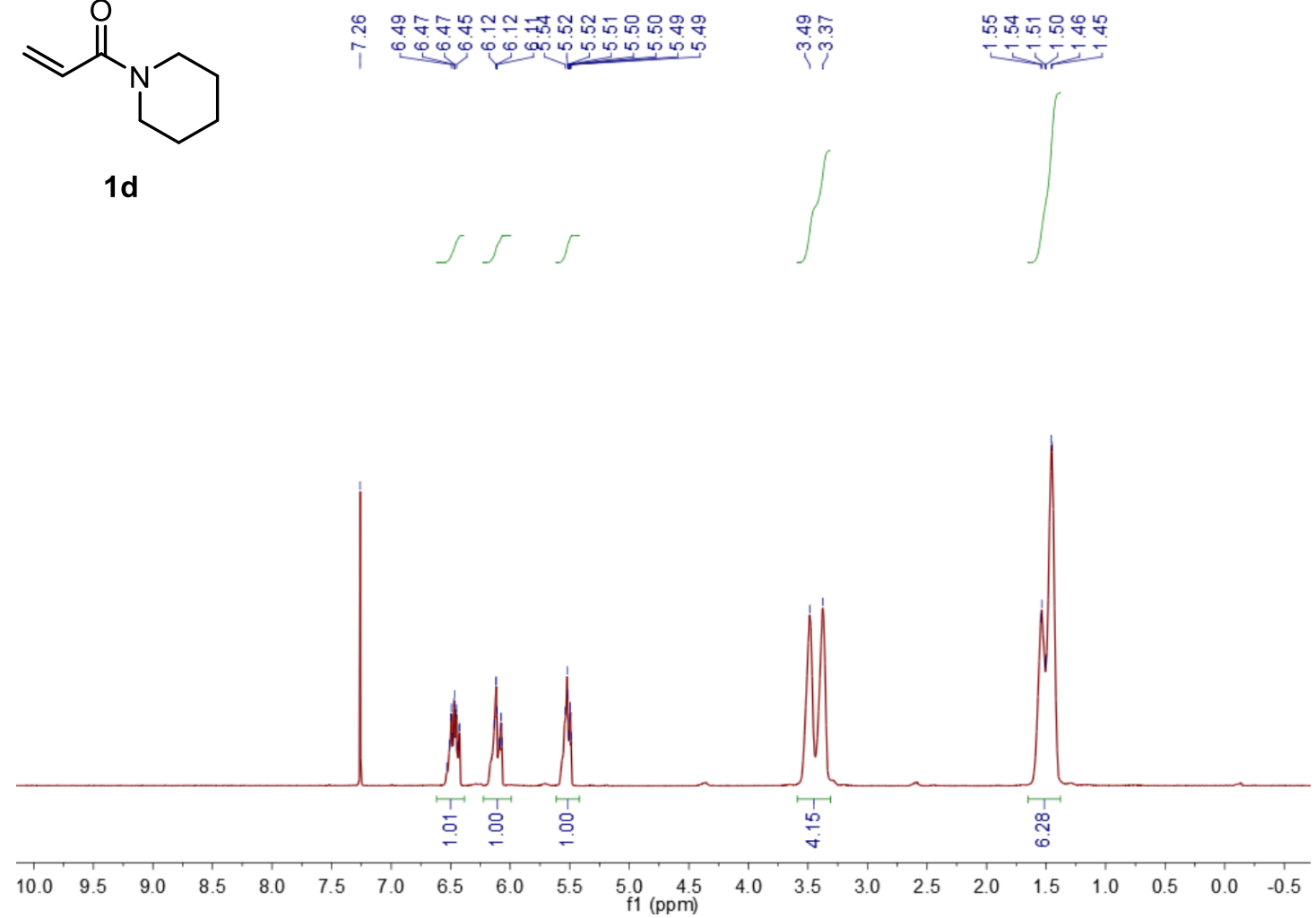

Figure S19. ${ }^{1} \mathrm{H} \mathrm{NMR}\left(400 \mathrm{MHz}, \mathrm{CDCl}_{3}\right)$ of $\mathbf{1 d}$.
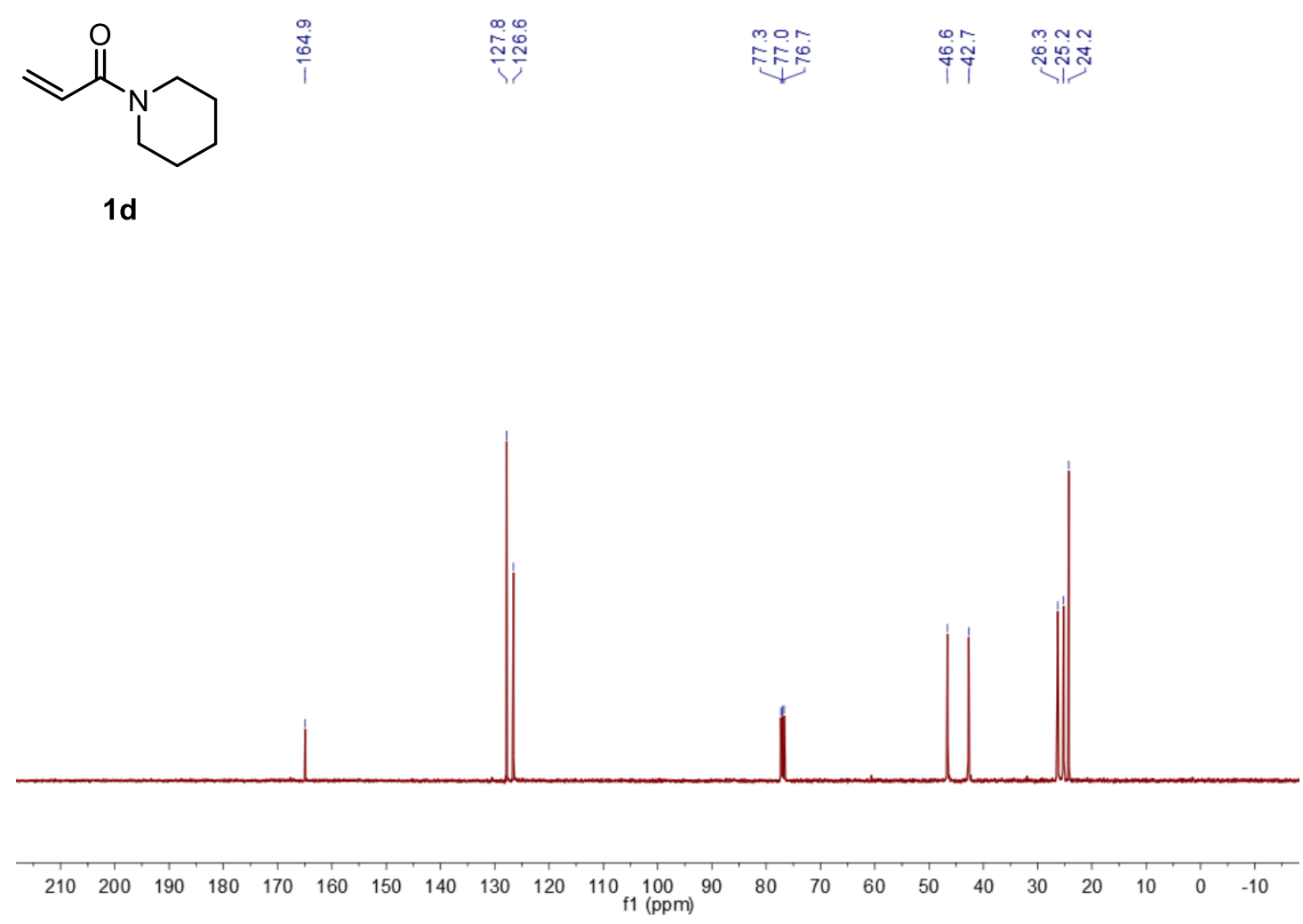

Figure S20. ${ }^{13} \mathrm{C} \mathrm{NMR}\left(101 \mathrm{MHz}, \mathrm{CDCl}_{3}\right)$ of $\mathbf{1 d}$. 
<smiles>C=CC(=O)N1CCOCC1</smiles>

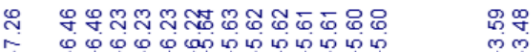

$1 e$

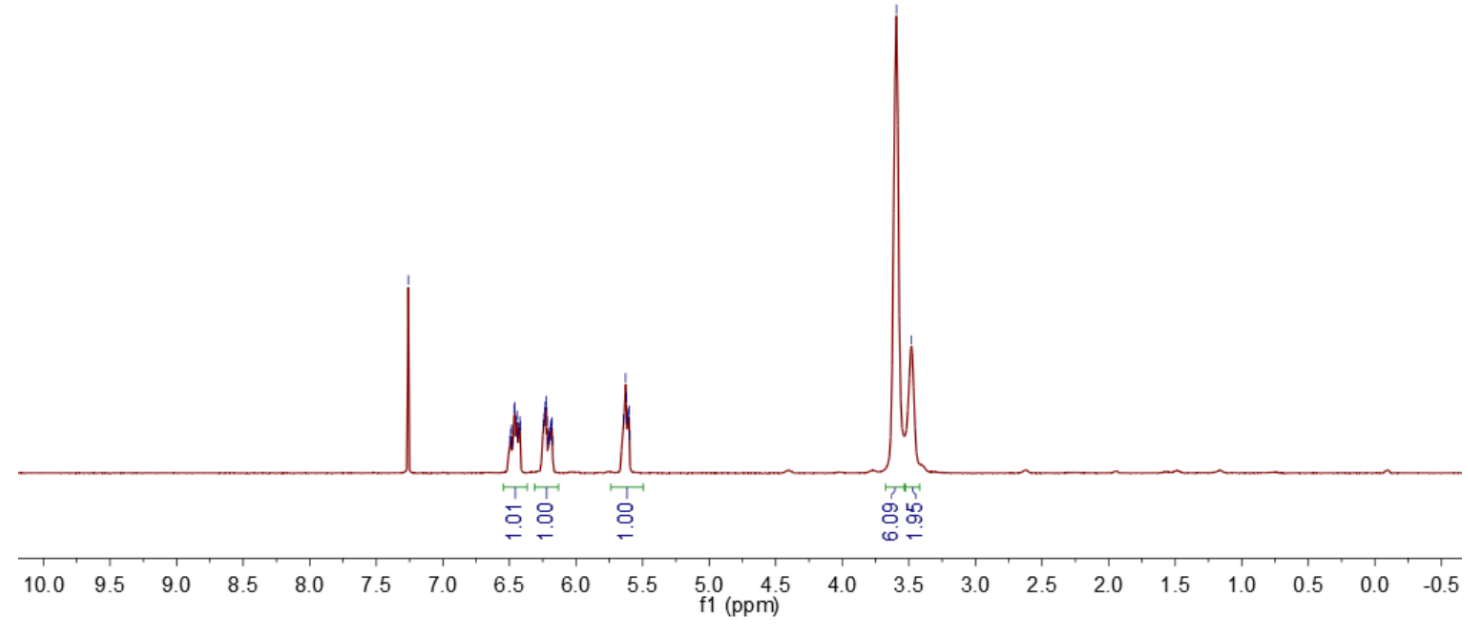

Figure S21. ${ }^{1} \mathrm{H} \mathrm{NMR}\left(400 \mathrm{MHz}, \mathrm{CDCl}_{3}\right)$ of $\mathbf{1 e}$<smiles>C=CC(=O)N1CCOCC1</smiles>

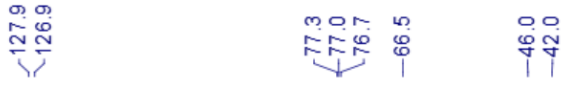

1e

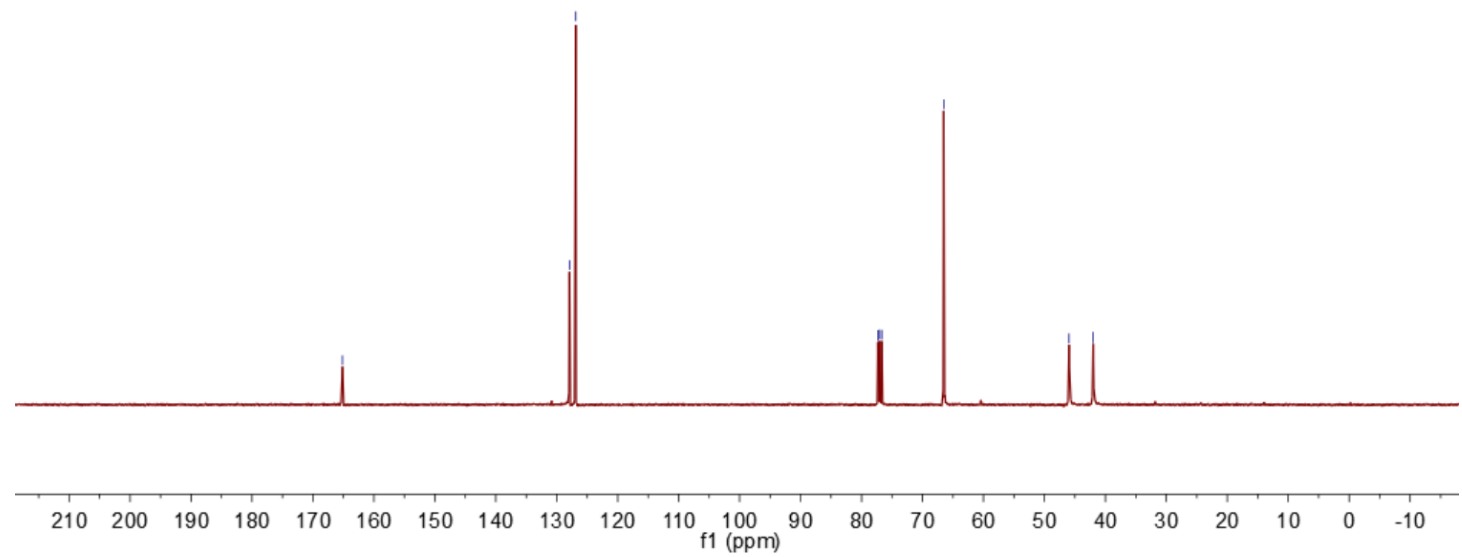

Figure S22. ${ }^{13} \mathrm{C} \mathrm{NMR}\left(101 \mathrm{MHz}, \mathrm{CDCl}_{3}\right)$ of $\mathbf{1 d}$. 


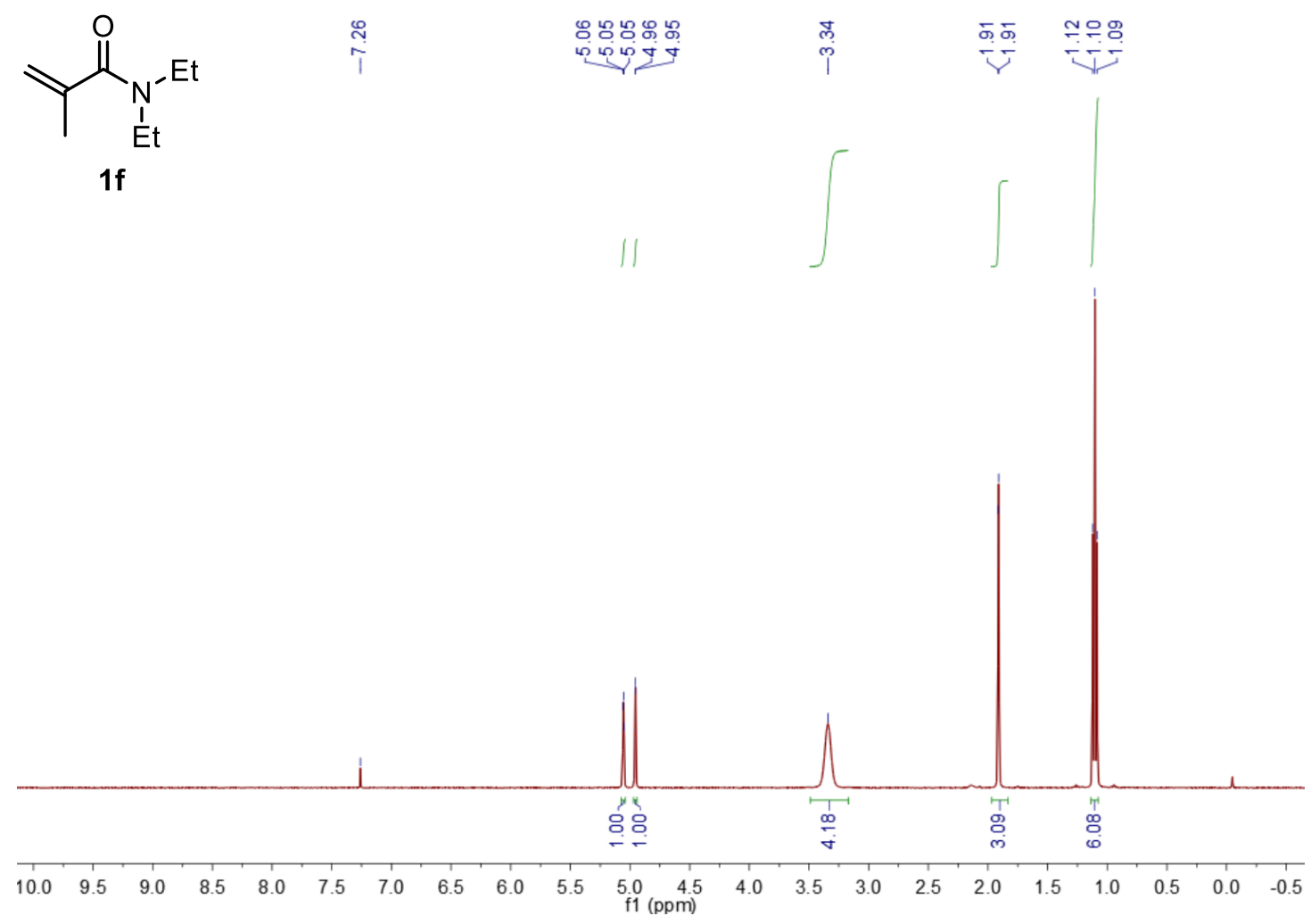

Figure S23. ${ }^{1} \mathrm{H}$ NMR $\left(400 \mathrm{MHz}, \mathrm{CDCl}_{3}\right)$ of $\mathbf{1 f}$.
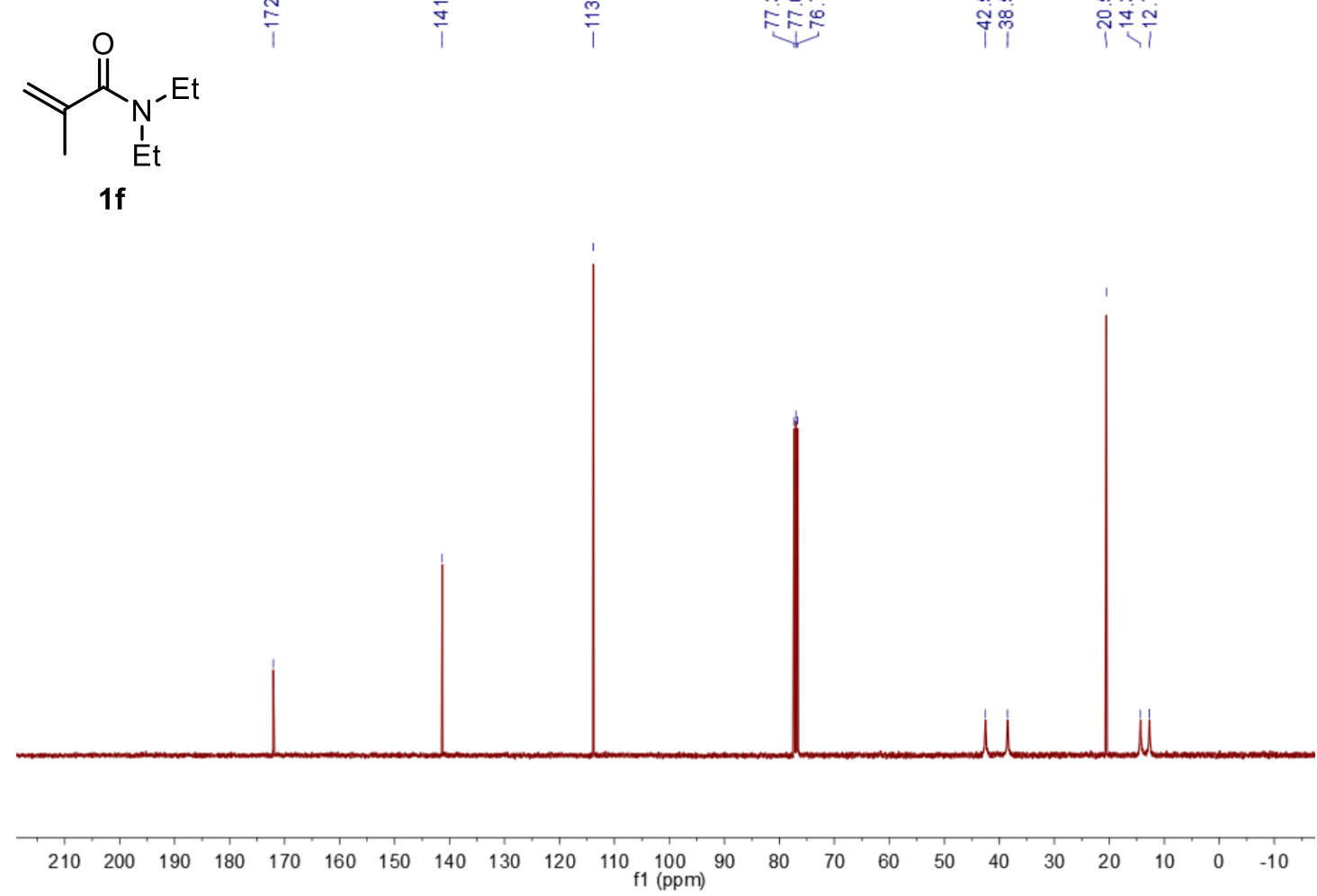

Figure S24. ${ }^{13} \mathrm{C}$ NMR $\left(101 \mathrm{MHz}, \mathrm{CDCl}_{3}\right)$ of $\mathbf{1 f}$. 


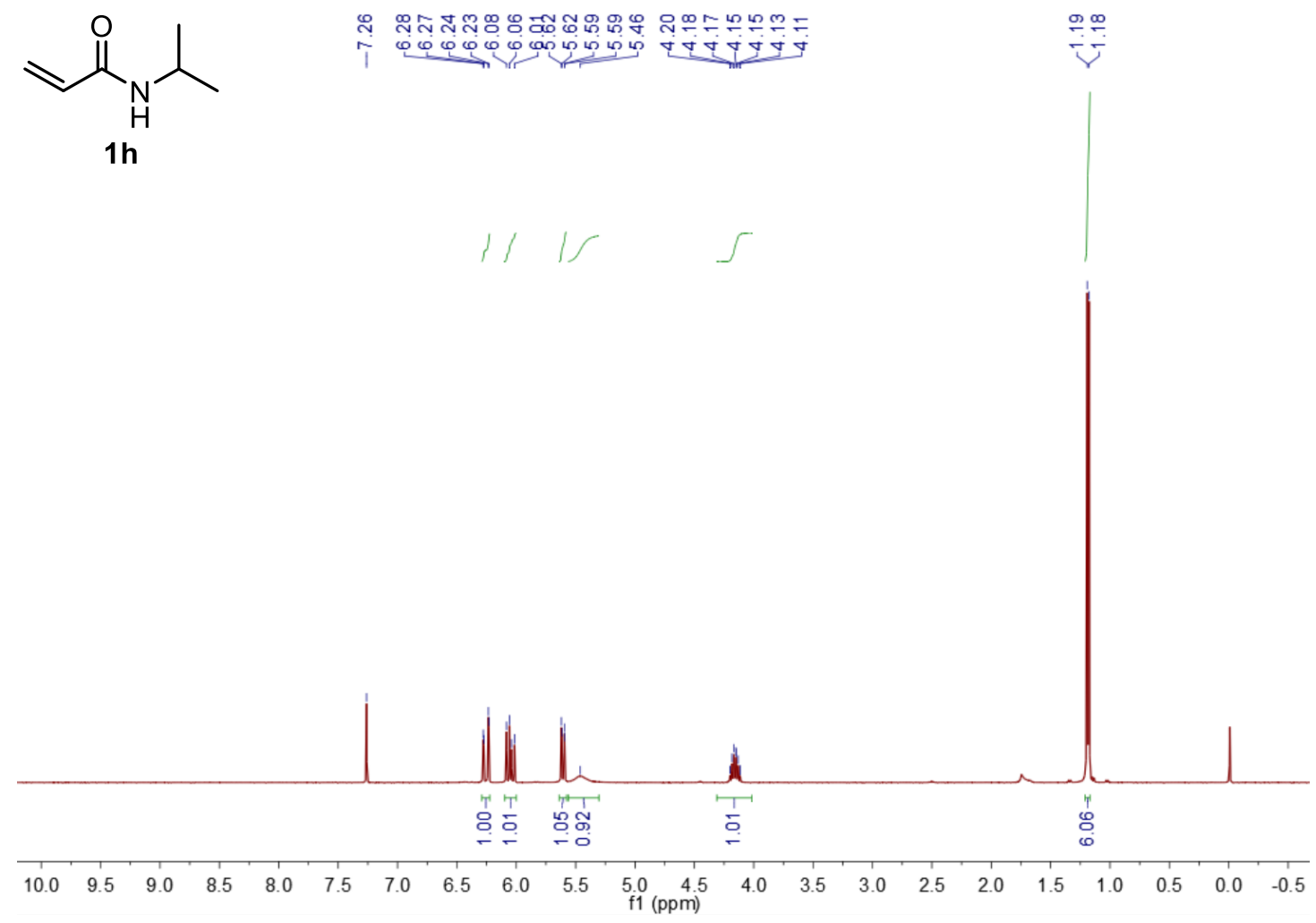

Figure S25. ${ }^{1} \mathrm{H}$ NMR $\left(400 \mathrm{MHz}, \mathrm{CDCl}_{3}\right)$ of $\mathbf{1 h}$.

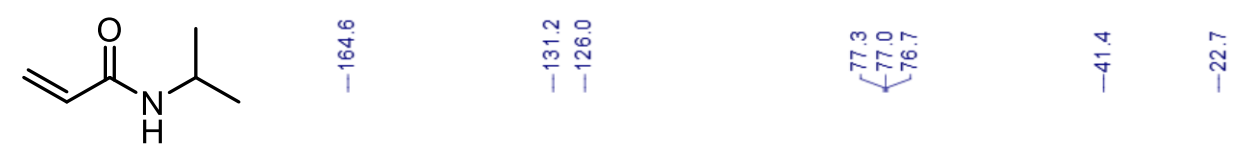

$1 \mathrm{~h}$

$\begin{array}{llllllllllllllllllllllllll}210 & 200 & 190 & 180 & 170 & 160 & 150 & 140 & 130 & 120 & 110 & 100 & 90 & 80 & 70 & 60 & 50 & 40 & 30 & 20 & 10 & 0 & -10\end{array}$

Figure S26. ${ }^{13} \mathrm{C}$ NMR $\left(101 \mathrm{MHz}, \mathrm{CDCl}_{3}\right)$ of $\mathbf{1 h}$. 


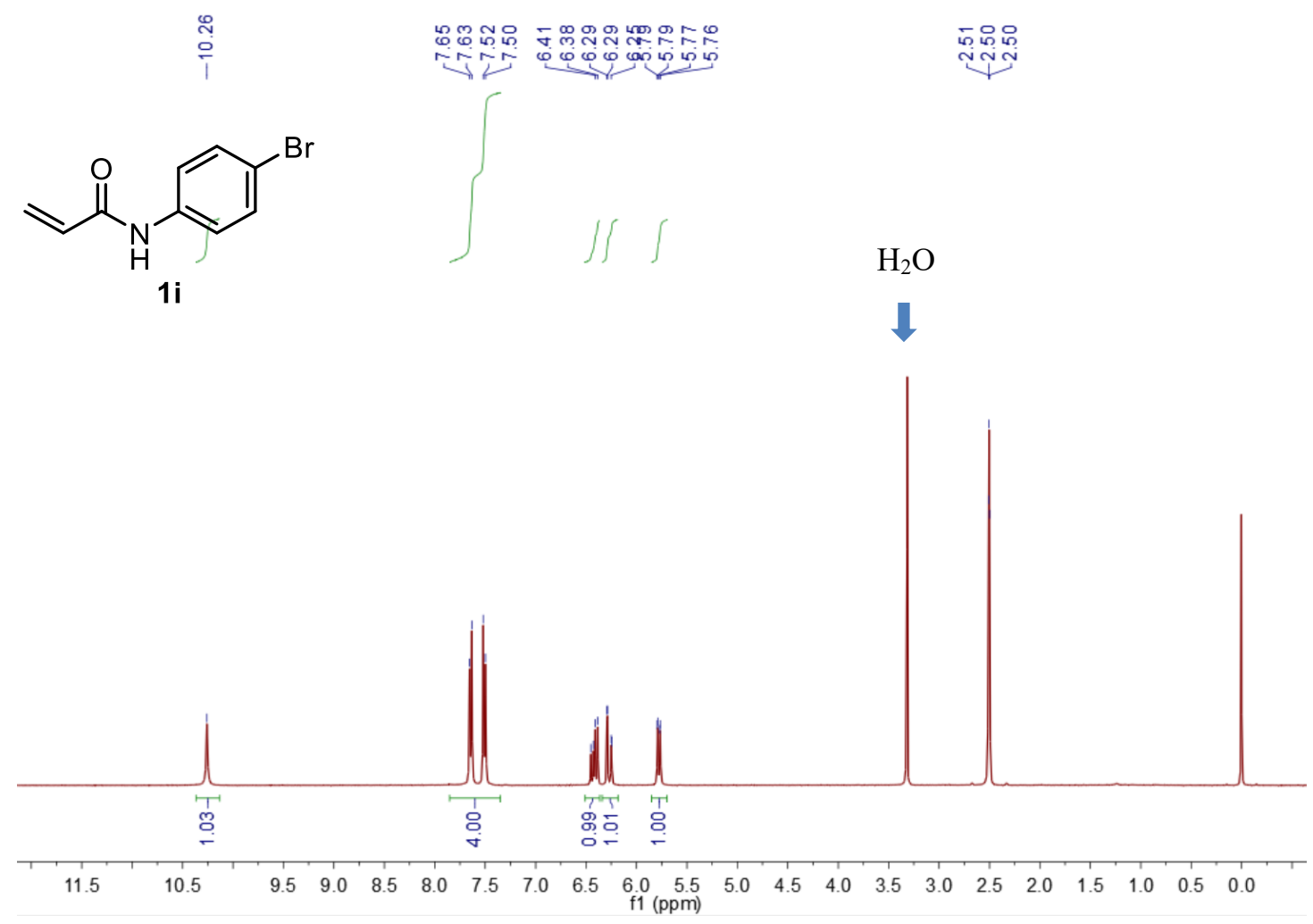

Figure S27. ${ }^{1} \mathrm{H}$ NMR (400 MHz, $d_{6}$-DMSO) of $1 \mathbf{i}$.

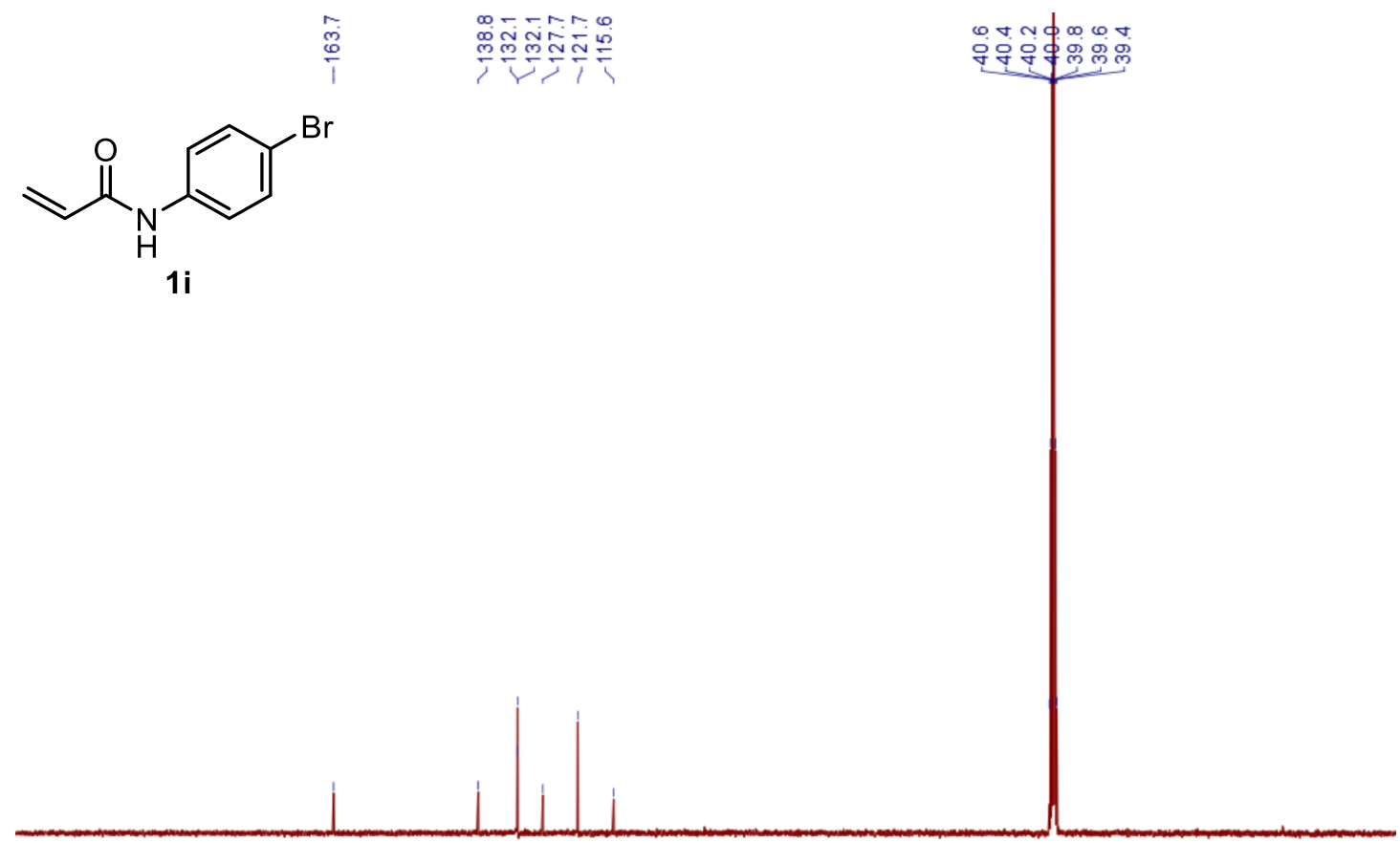

$\begin{array}{llllllllllllllllllllll}210 & 200 & 190 & 180 & 170 & 160 & 150 & 140 & 130 & 120 & 110 \begin{array}{l}100 \\ \mathrm{f} 1(\mathrm{ppm})\end{array} & 90 & 80 & 70 & 60 & 50 & 40 & 30 & 20 & 10 & 0 & -10\end{array}$

Figure S28. ${ }^{13} \mathrm{C}$ NMR (101 MHz, $d_{6}$-DMSO) of $\mathbf{1 i}$. 

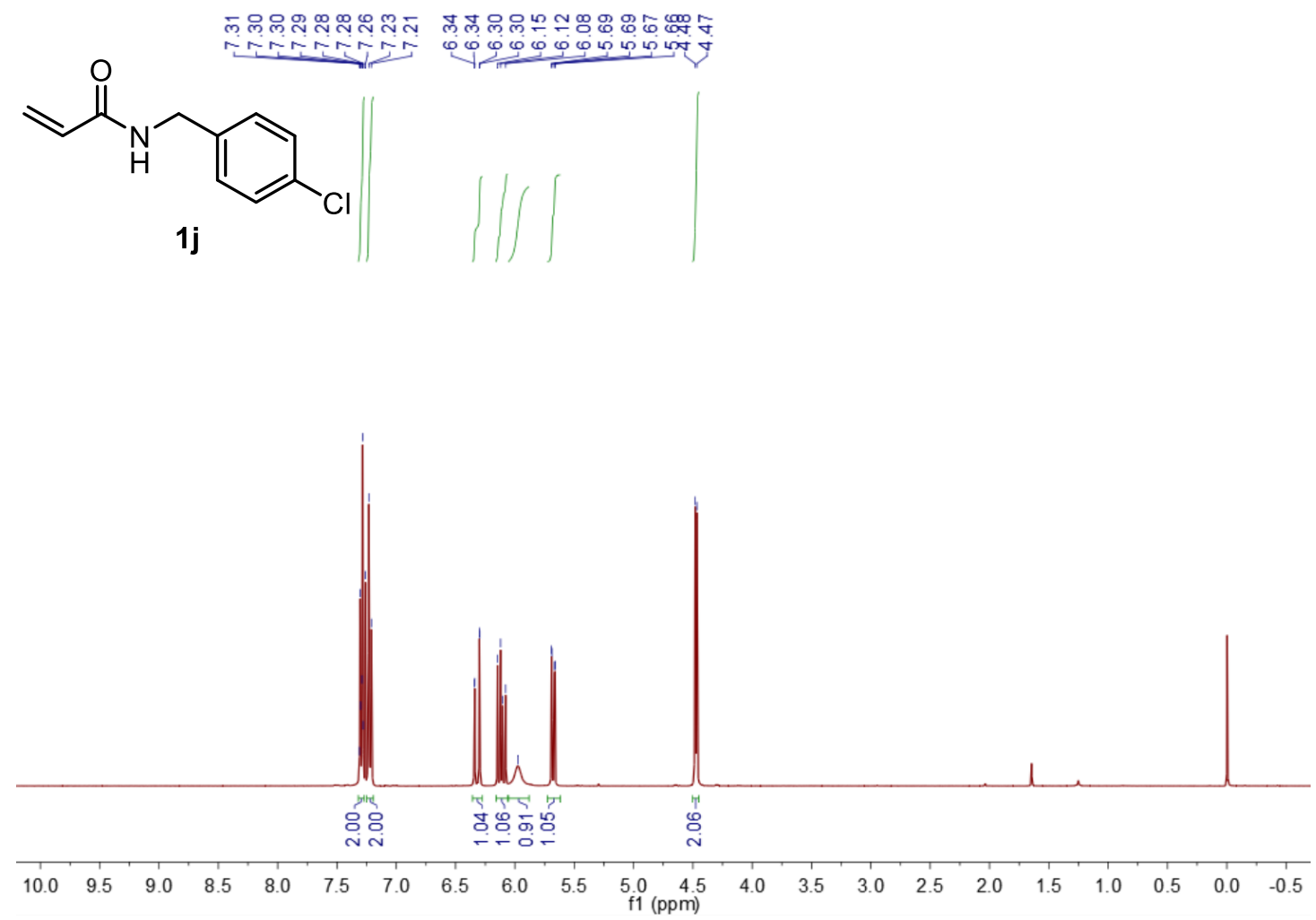

Figure S29. ${ }^{1} \mathrm{H}$ NMR $\left(400 \mathrm{MHz}, \mathrm{CDCl}_{3}\right)$ of $\mathbf{1 j}$.

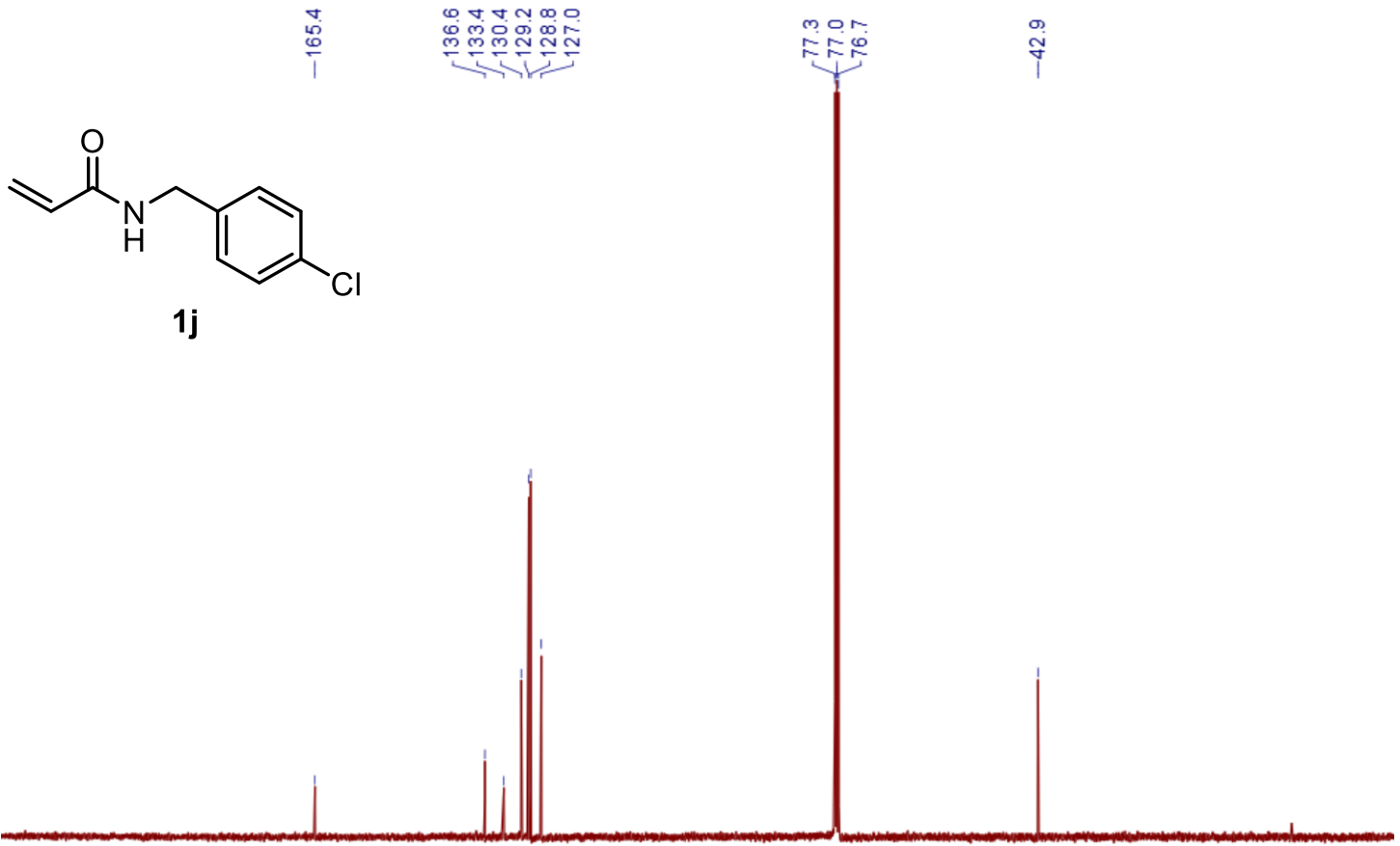

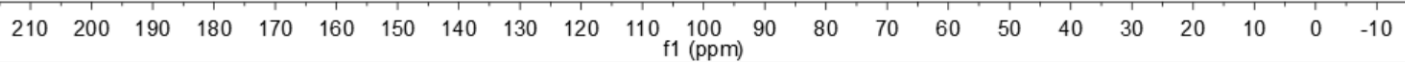

Figure S30. ${ }^{13} \mathrm{C}$ NMR $\left(101 \mathrm{MHz}, \mathrm{CDCl}_{3}\right)$ of $\mathbf{1 j}$. 


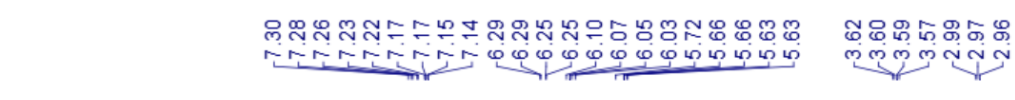<smiles>C=CC(=O)NCCc1cc(Cl)ccc1Cl</smiles>

$1 \mathrm{k}$

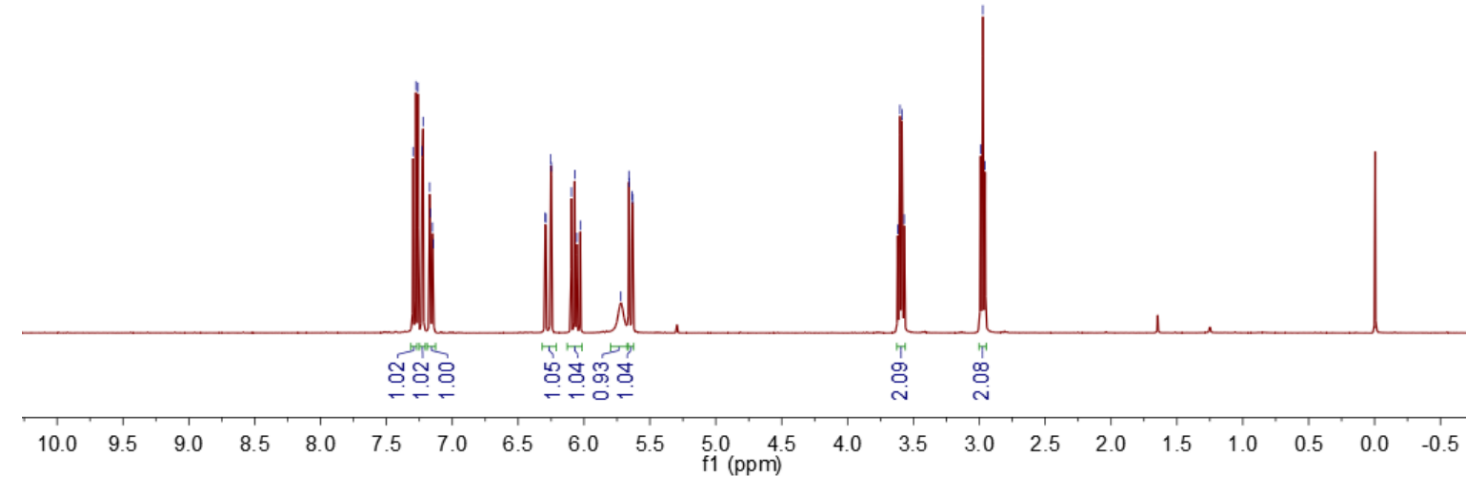

Figure S31. ${ }^{1} \mathrm{H}$ NMR (400 MHz, $\mathrm{CDCl}_{3}$ ) of $\mathbf{1 k}$.

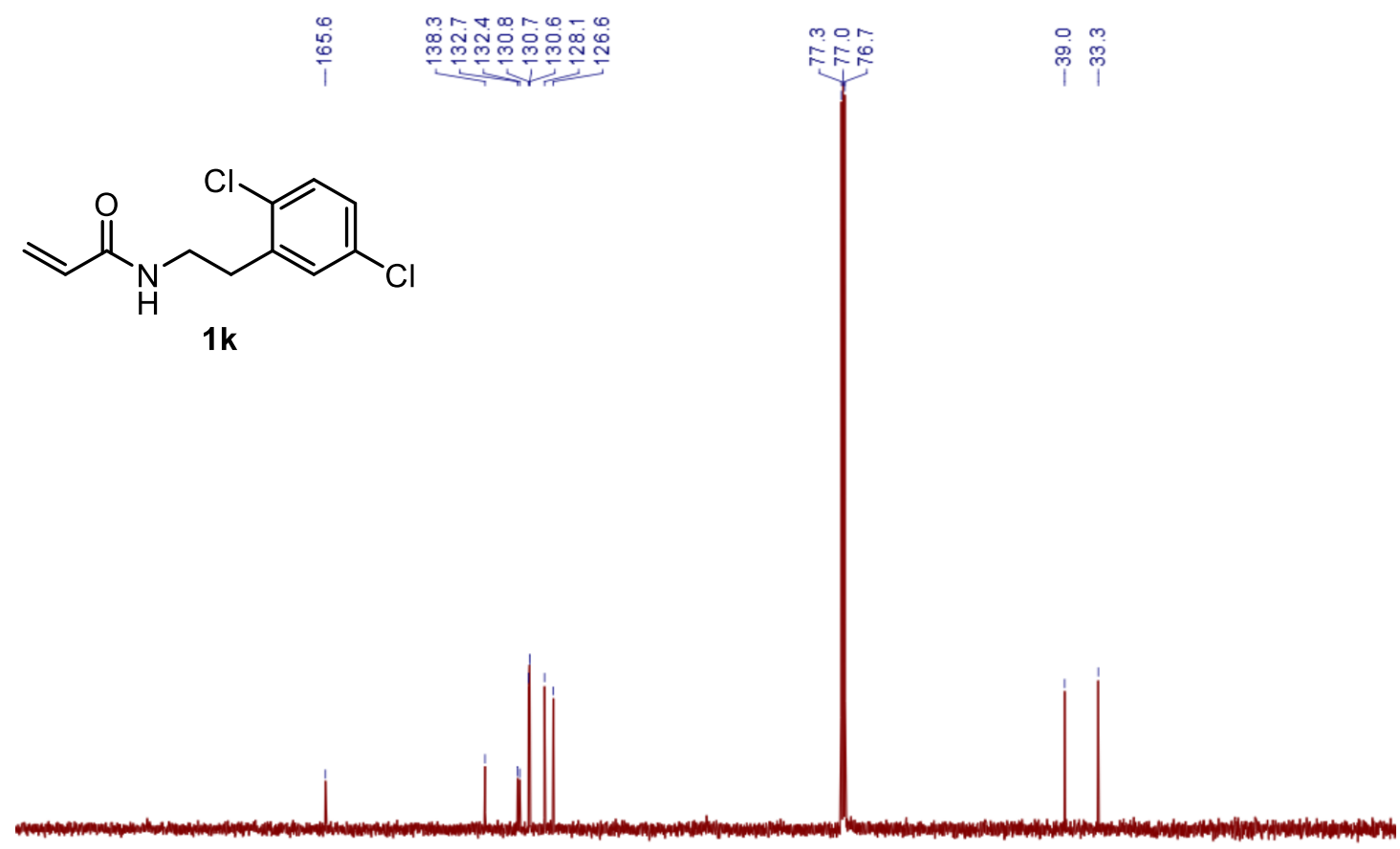

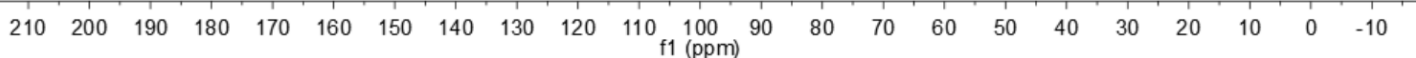

Figure S32. ${ }^{13} \mathrm{C}$ NMR $\left(101 \mathrm{MHz}, \mathrm{CDCl}_{3}\right)$ of $\mathbf{1 k}$. 


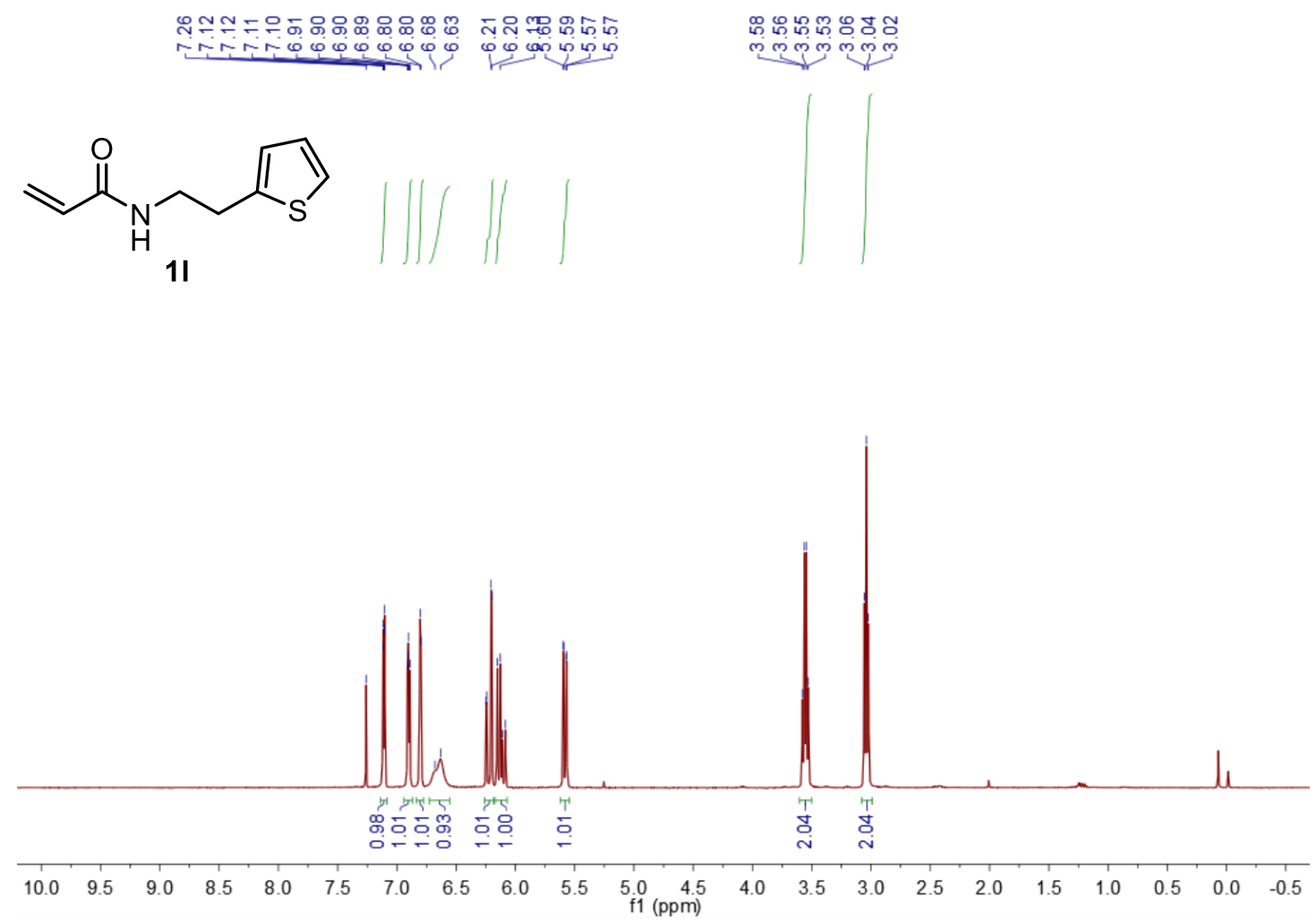

Figure S33. ${ }^{1} \mathrm{H}$ NMR (400 MHz, $\left.\mathrm{CDCl}_{3}\right)$ of 11.

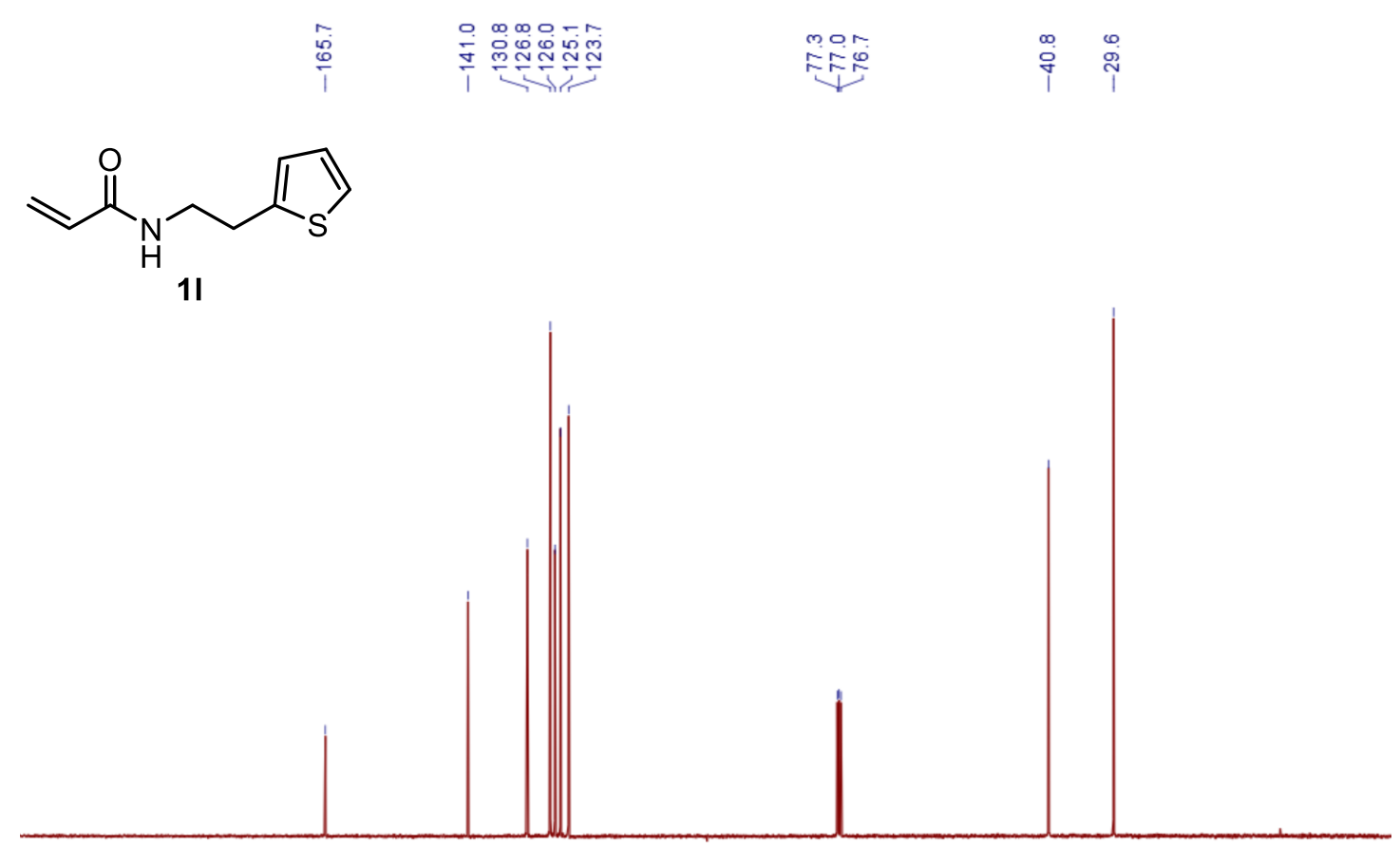

$\begin{array}{llllllllllllllllllllll}210 & 200 & 190 & 180 & 170 & 160 & 150 & 140 & 130 & 120 & 110 \begin{array}{c}100 \\ \mathrm{f} 1(\mathrm{ppm})\end{array} & 90 & 80 & 70 & 60 & 50 & 40 & 30 & 20 & 10 & 0 & -10\end{array}$

Figure S34. ${ }^{13} \mathrm{C}$ NMR (101 MHz, $\left.\mathrm{CDCl}_{3}\right)$ of 11 . 

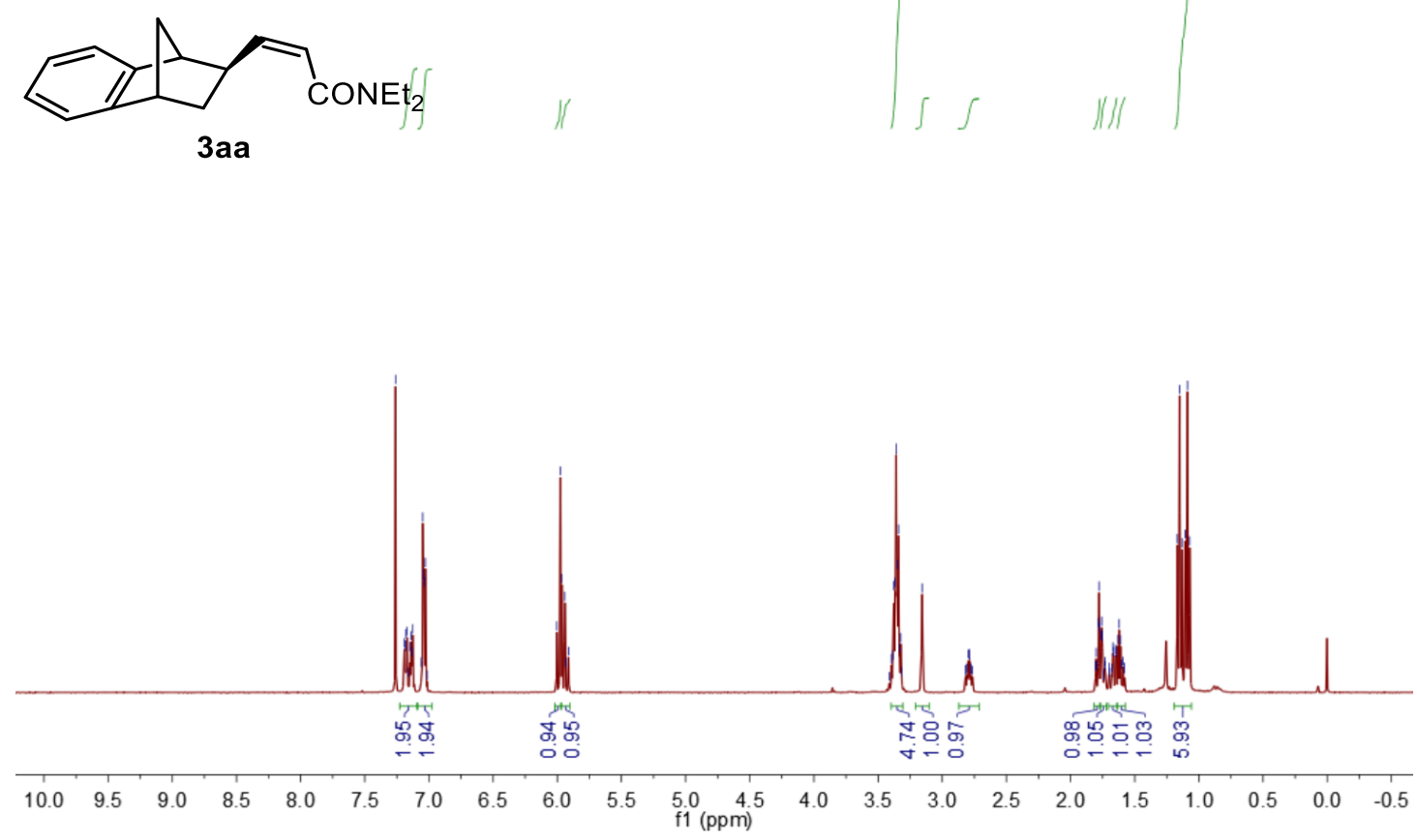

Figure S35. ${ }^{1} \mathrm{H}$ NMR $\left(400 \mathrm{MHz}, \mathrm{CDCl}_{3}\right)$ of 3aa.

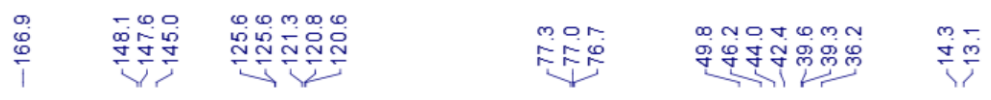
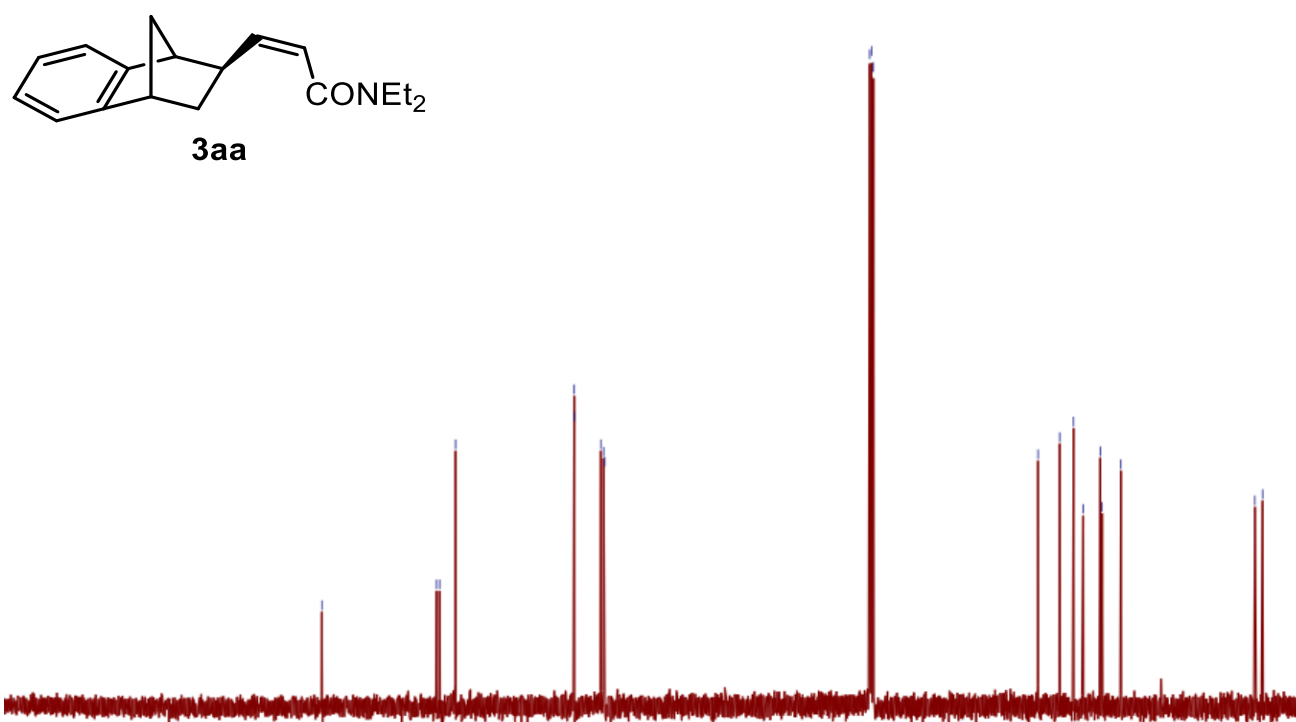

$\begin{array}{lllllllllllllllllllllll}210 & 200 & 190 & 180 & 170 & 160 & 150 & 140 & 130 & 120 & 110 & 100 & 90 & 80 & 70 & 60 & 50 & 40 & 30 & 20 & 10 & 0 & -10\end{array}$

Figure S36. ${ }^{13} \mathrm{C}$ NMR $\left(101 \mathrm{MHz}, \mathrm{CDCl}_{3}\right)$ of 3aa. 
$1 \mathrm{CONEt}_{2}$

$3 a b$

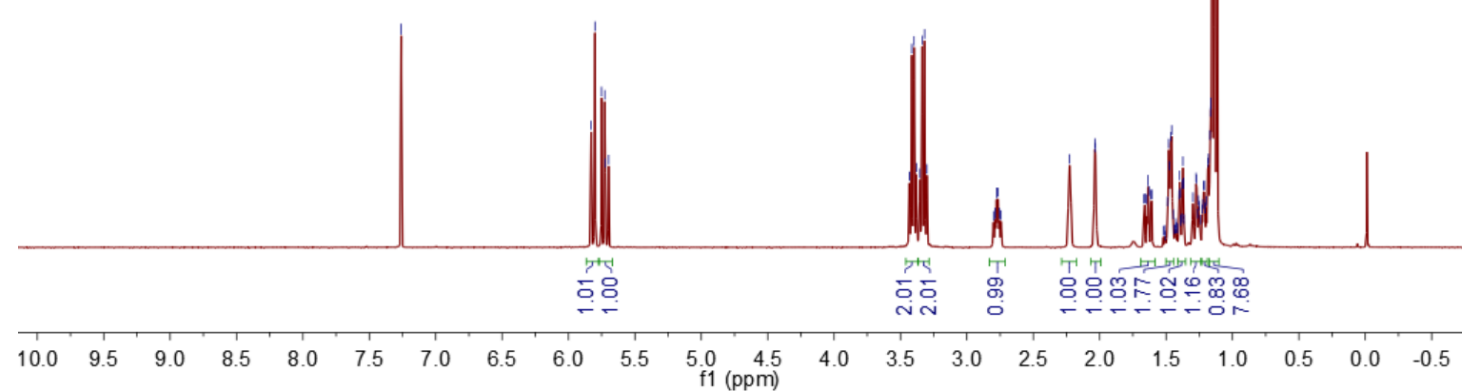

Figure S37. ${ }^{1} \mathrm{H} \mathrm{NMR}\left(400 \mathrm{MHz}, \mathrm{CDCl}_{3}\right)$ of $\mathbf{3 a b}$.

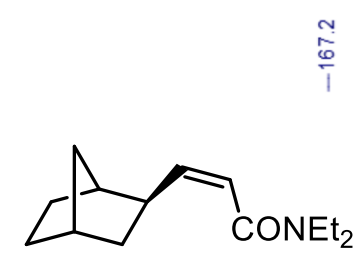

$3 a b$

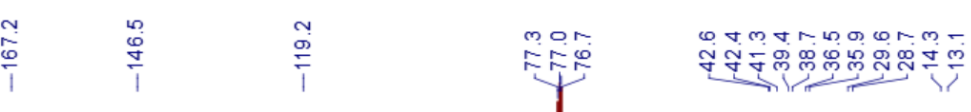

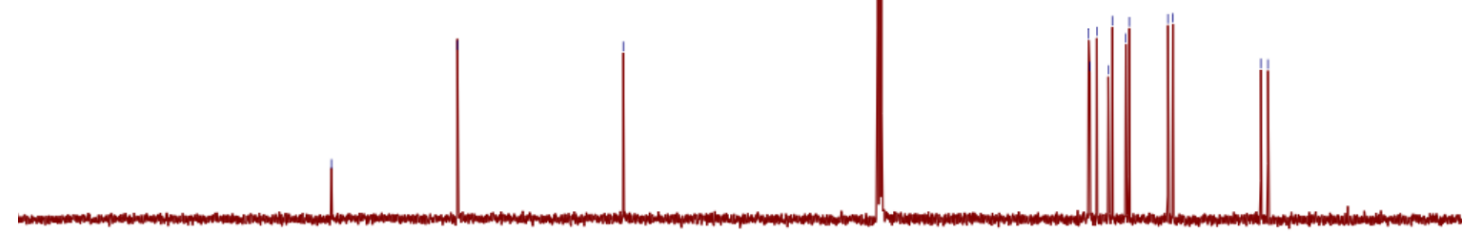

$\begin{array}{lllllllllllllllllllllll}210 & 200 & 190 & 180 & 170 & 160 & 150 & 140 & 130 & 120 & 110 & 100 & 90 & 80 & 70 & 60 & 50 & 40 & 30 & 20 & 10 & 0 & -10\end{array}$

Figure S38. ${ }^{13} \mathrm{C}$ NMR $\left(101 \mathrm{MHz}, \mathrm{CDCl}_{3}\right)$ of $\mathbf{3 a b}$. 

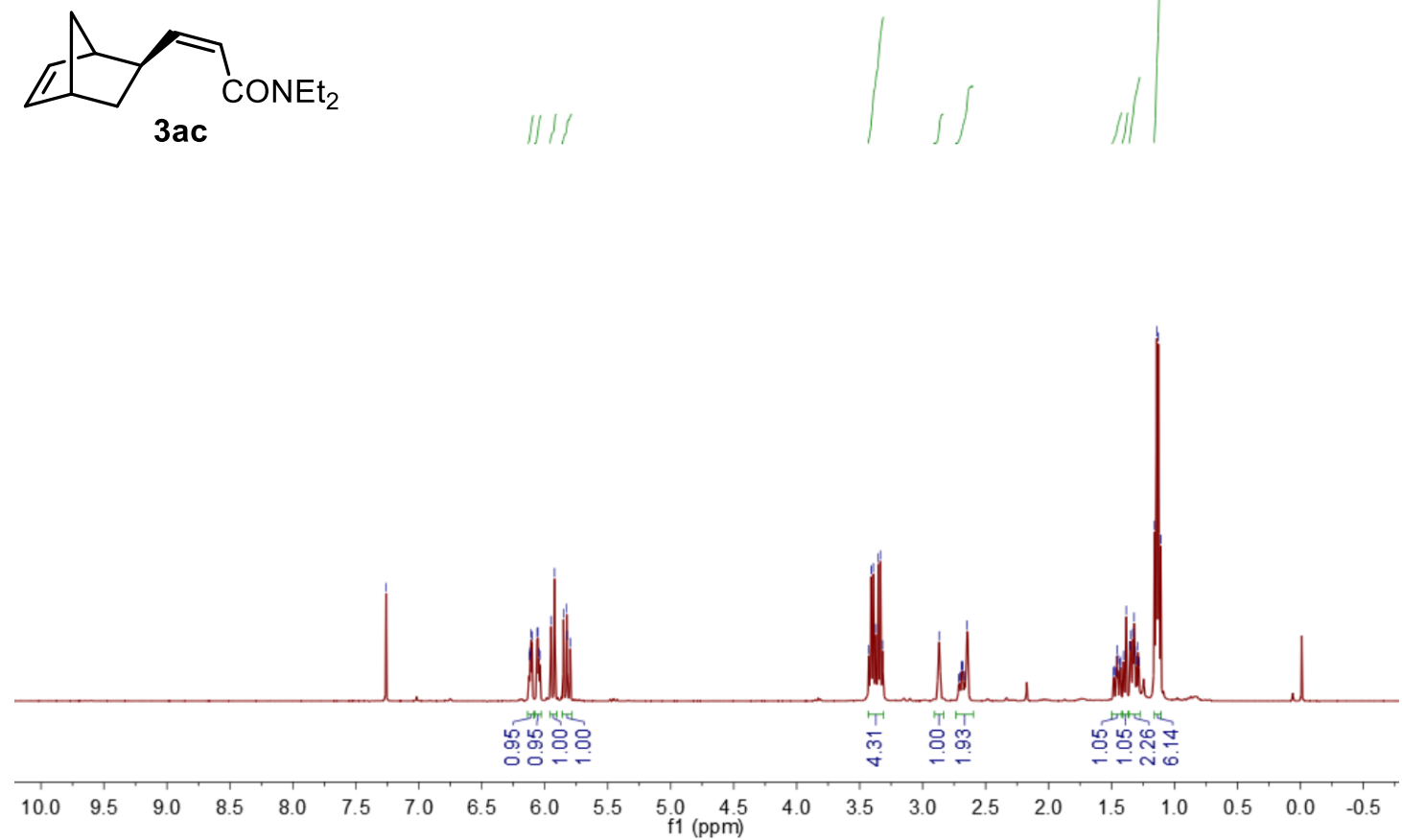

Figure S39. ${ }^{1} \mathrm{H}$ NMR $\left(400 \mathrm{MHz}, \mathrm{CDCl}_{3}\right)$ of 3ac.

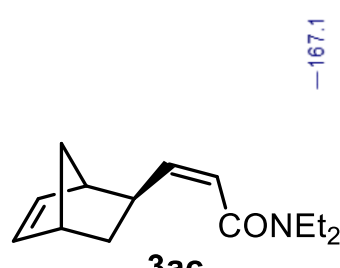

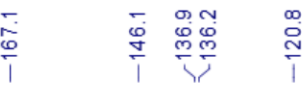

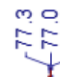

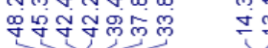

3ac

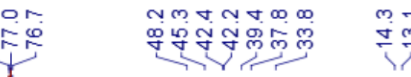

$\begin{array}{llllllllllllllllllllll}210 & 200 & 190 & 180 & 170 & 160 & 150 & 140 & 130 & 120 & 110 \underset{\mathrm{f} 1(\mathrm{ppm})}{100} & 90 & 80 & 70 & 60 & 50 & 40 & 30 & 20 & 10 & 0 & -10\end{array}$

Figure S40. ${ }^{13} \mathrm{C} \mathrm{NMR}\left(101 \mathrm{MHz}, \mathrm{CDCl}_{3}\right)$ of 3ac. 


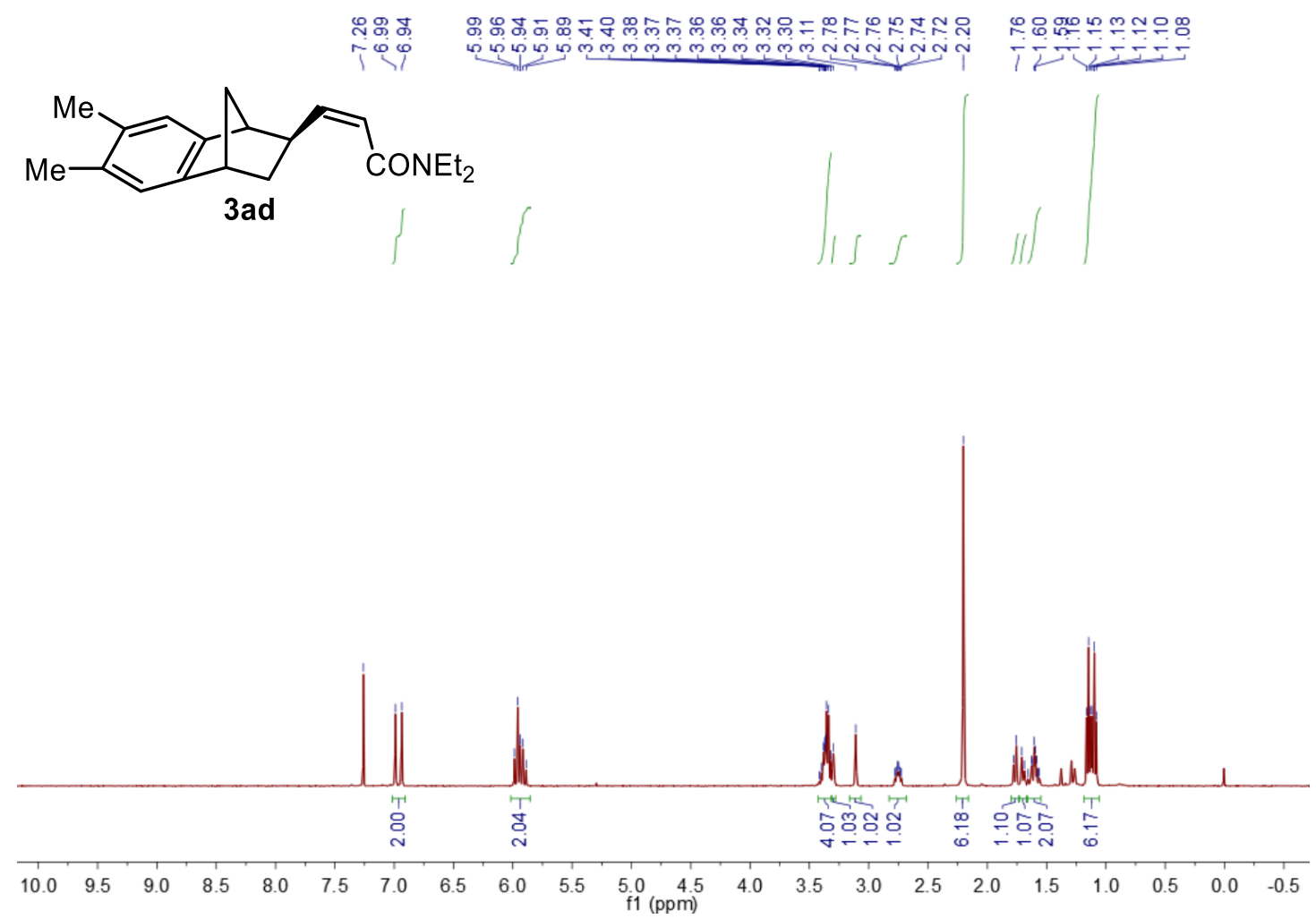

Figure S41. ${ }^{1} \mathrm{H}$ NMR $\left(400 \mathrm{MHz}, \mathrm{CDCl}_{3}\right)$ of $\mathbf{3 a d}$.
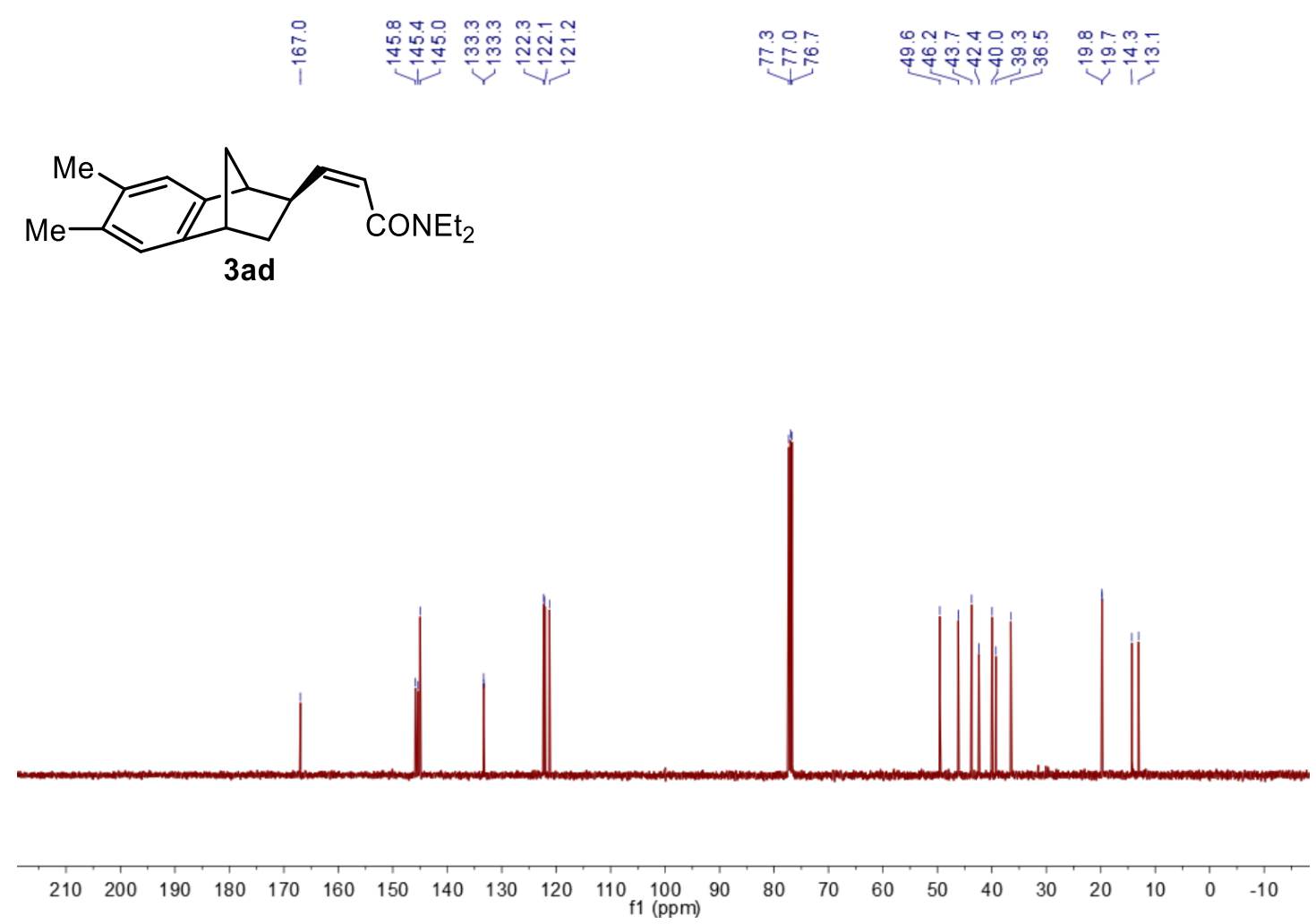

Figure $\mathbf{S 4 2} .{ }^{13} \mathrm{C}$ NMR $\left(101 \mathrm{MHz}, \mathrm{CDCl}_{3}\right)$ of $\mathbf{3 a d}$. 

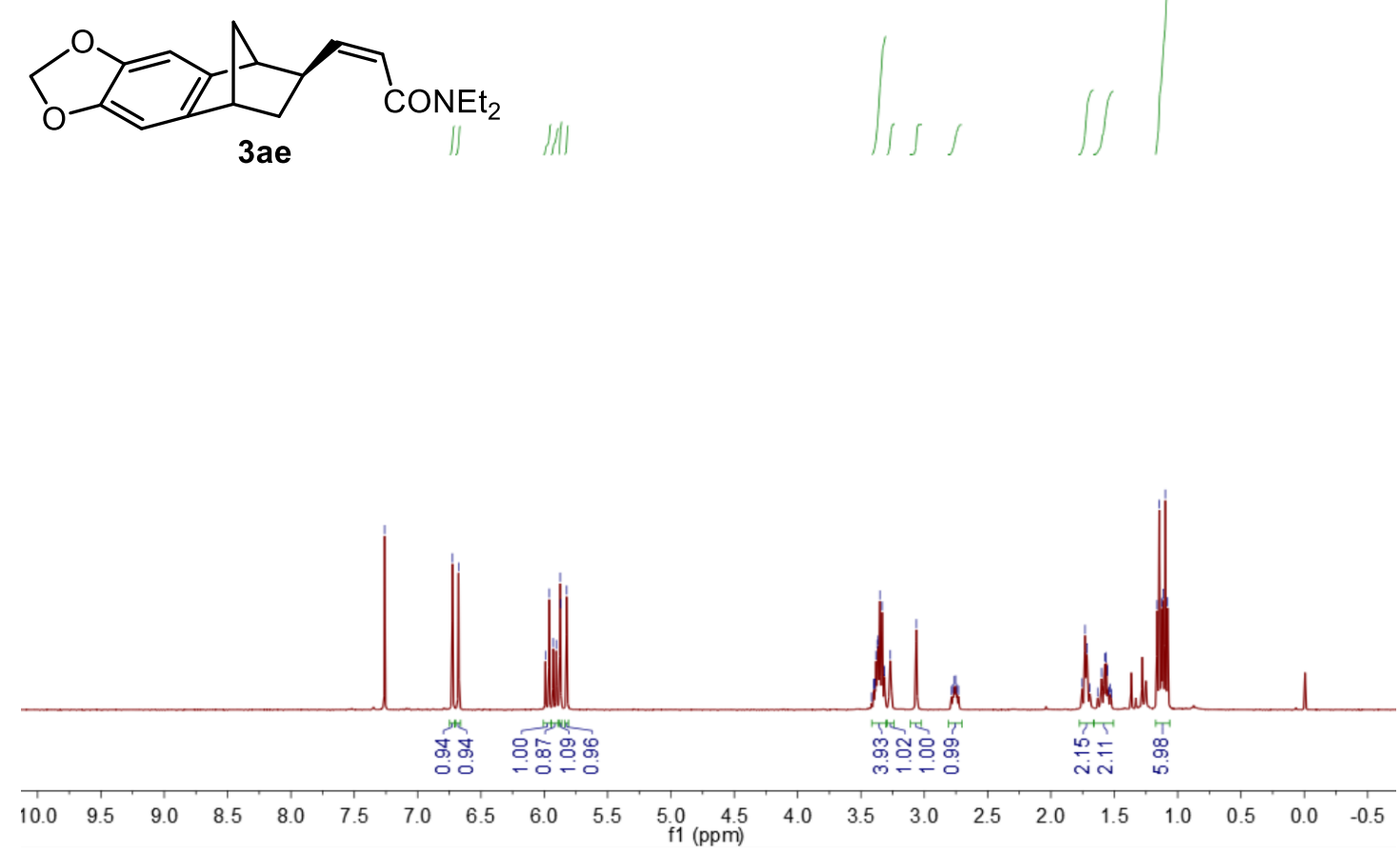

Figure S43. ${ }^{1} \mathrm{H}$ NMR $\left(400 \mathrm{MHz}, \mathrm{CDCl}_{3}\right)$ of 3ae.
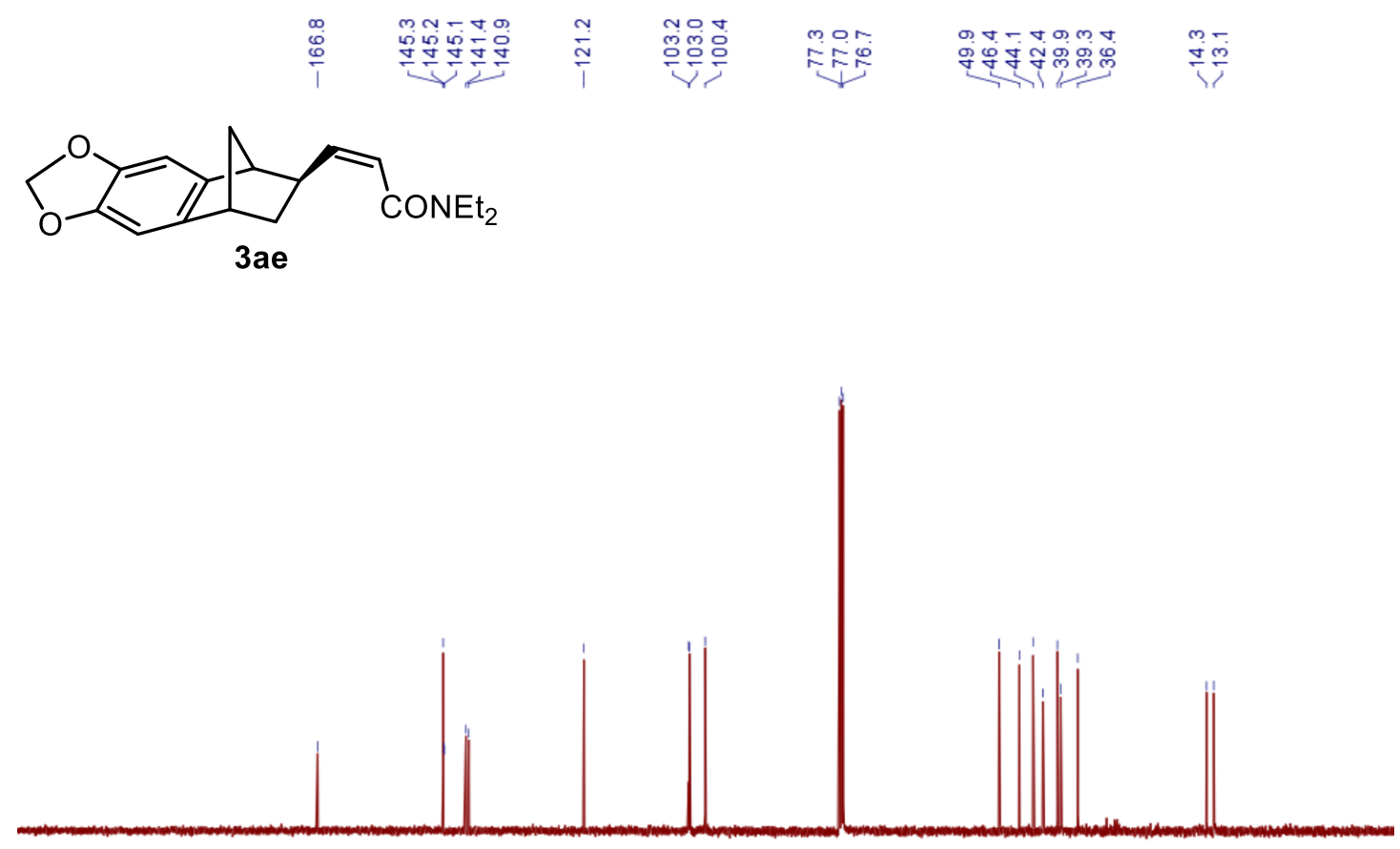

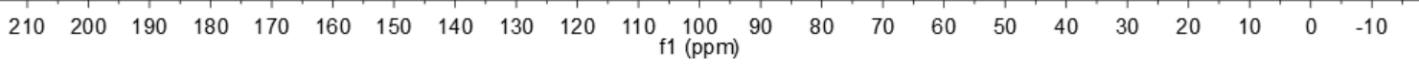

Figure S44. ${ }^{13} \mathrm{C}$ NMR (101 MHz, $\left.\mathrm{CDCl}_{3}\right)$ of 3ae. 


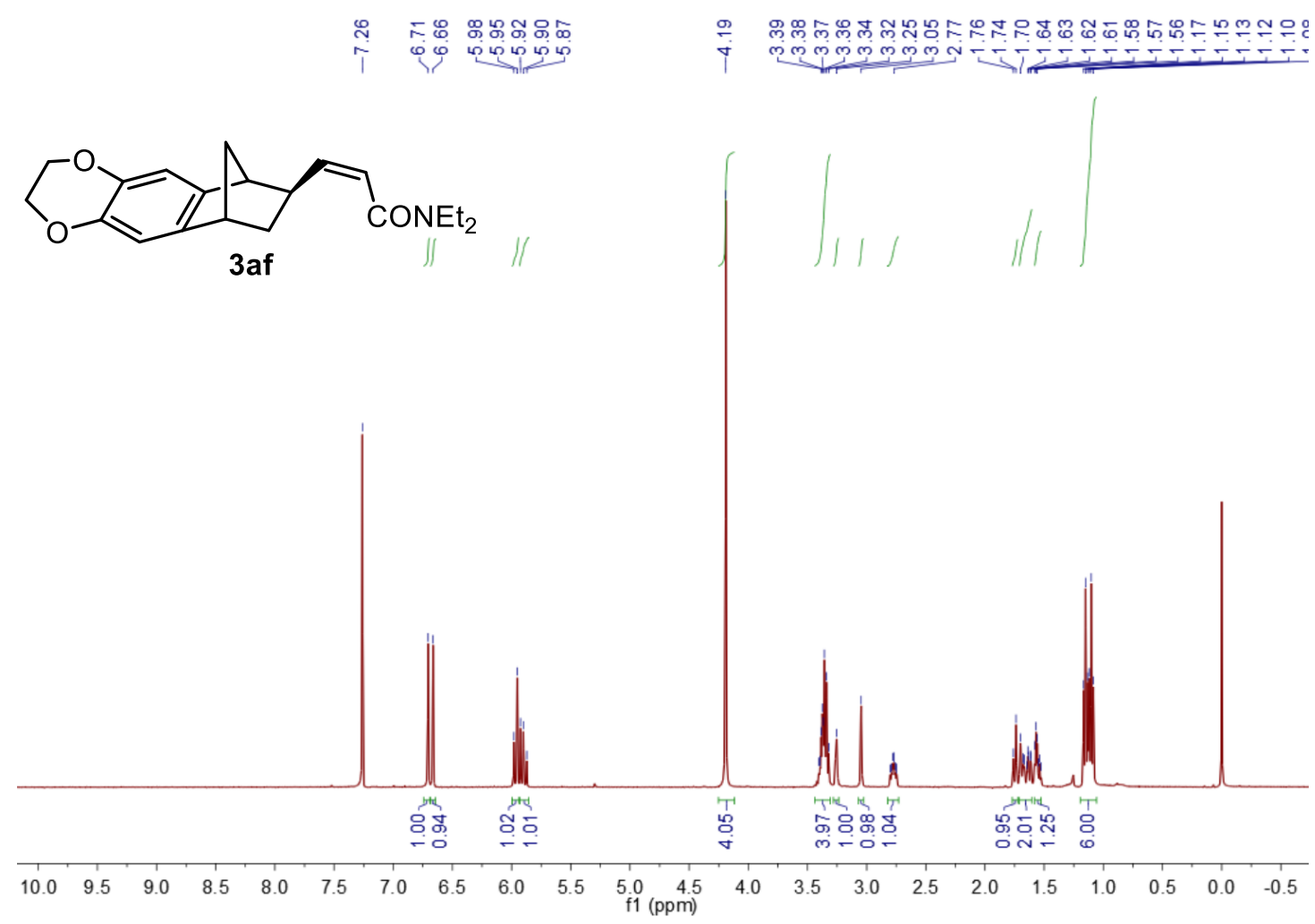

Figure $\mathbf{S 4 5} .{ }^{1} \mathrm{H}$ NMR $\left(400 \mathrm{MHz}, \mathrm{CDCl}_{3}\right)$ of $\mathbf{3 a f}$.

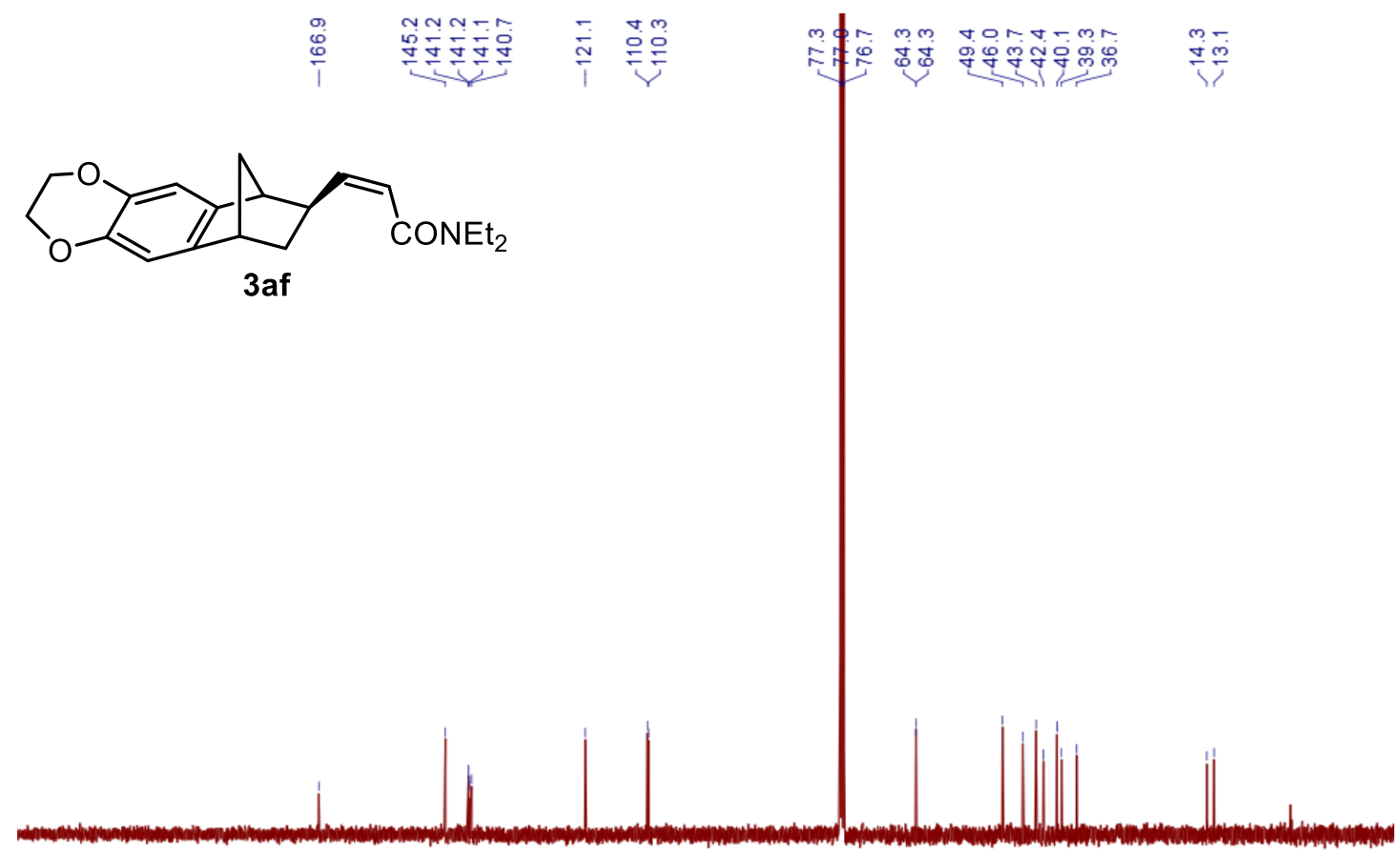

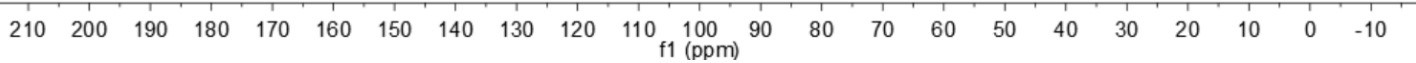

Figure S46. ${ }^{13} \mathrm{C}$ NMR $\left(101 \mathrm{MHz}, \mathrm{CDCl}_{3}\right)$ of 3af. 


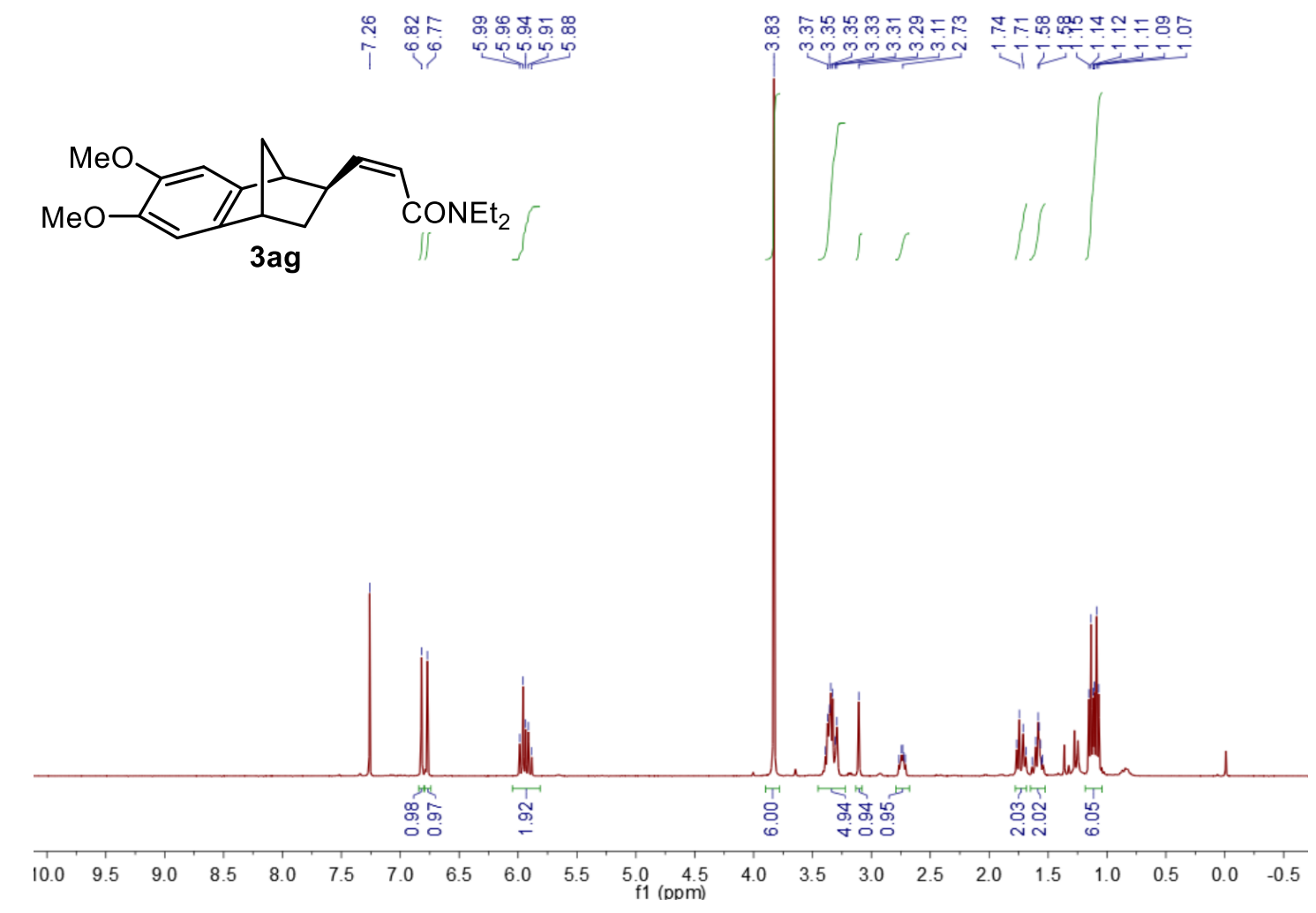

Figure S47. ${ }^{1} \mathrm{H} \mathrm{NMR}\left(400 \mathrm{MHz}, \mathrm{CDCl}_{3}\right)$ of $\mathbf{3 a g}$.

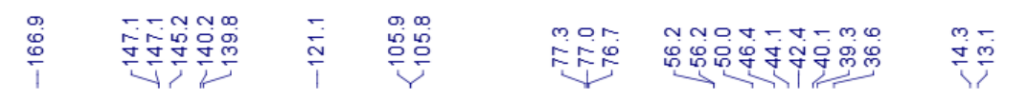<smiles>CCOC(=O)/C=C\[C@H]1C[C@@H](C)c2cc(OC)c(OC)cc21</smiles>

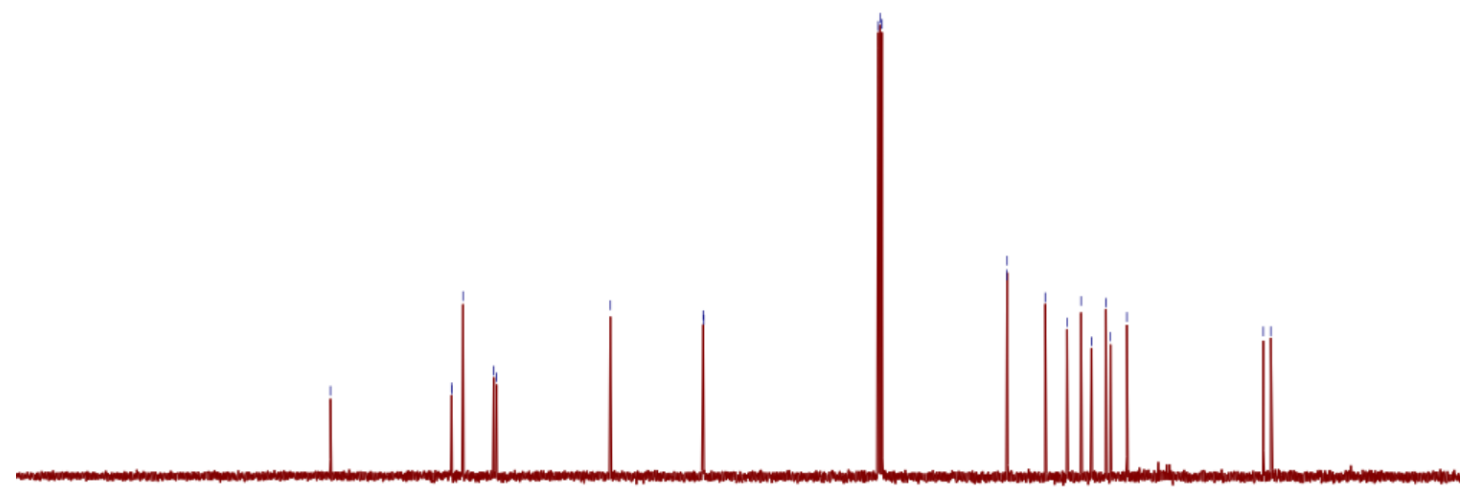

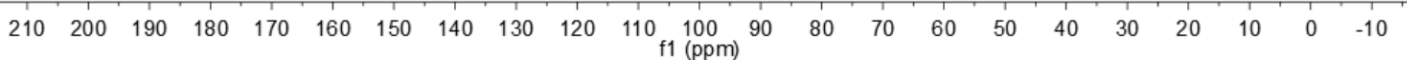

Figure $\mathbf{S 4 8} .{ }^{13} \mathrm{C}$ NMR $\left(101 \mathrm{MHz}, \mathrm{CDCl}_{3}\right)$ of $\mathbf{3 a g}$. 


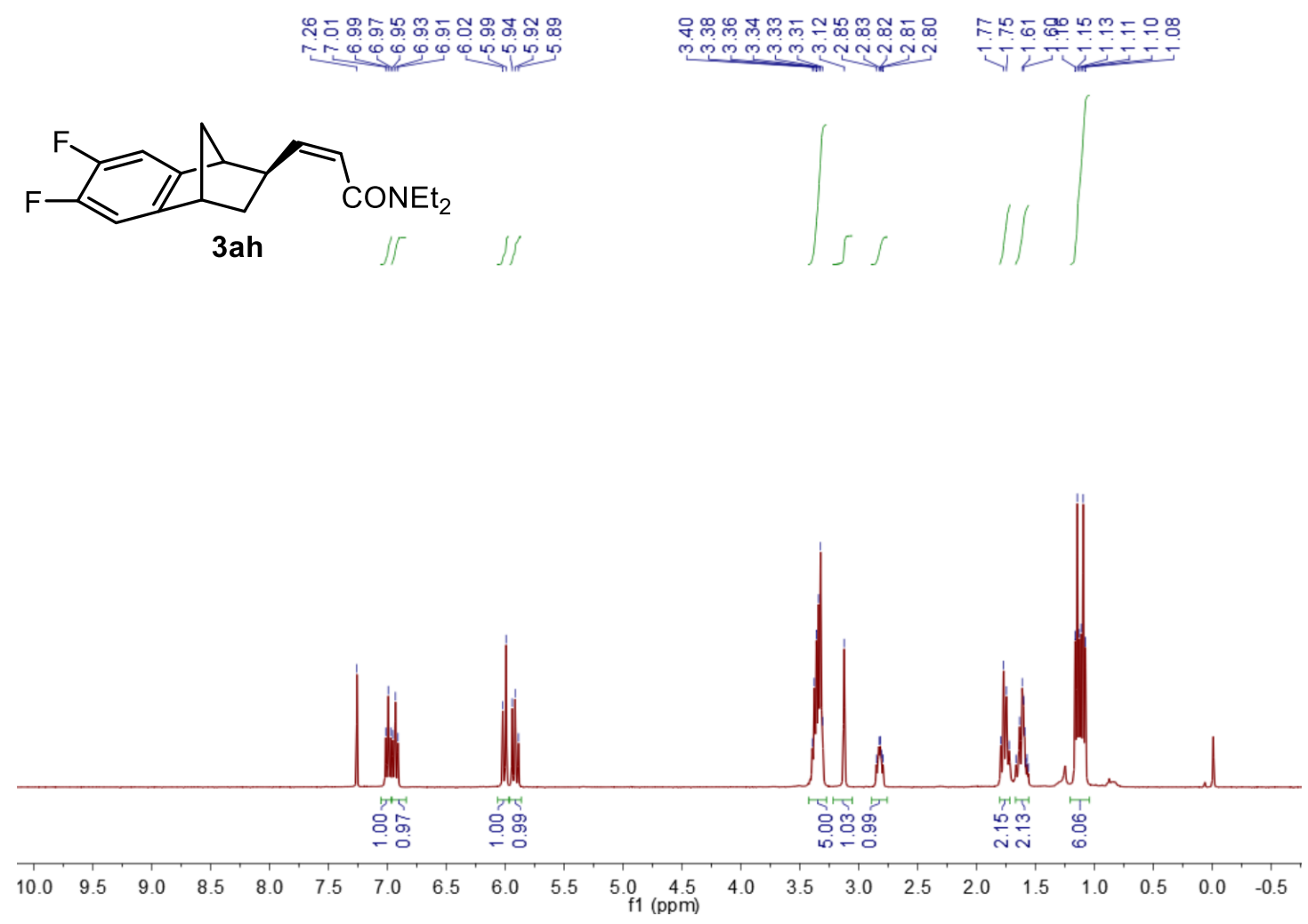

Figure S49. ${ }^{1} \mathrm{H} \mathrm{NMR}\left(400 \mathrm{MHz}, \mathrm{CDCl}_{3}\right)$ of $\mathbf{3 a h}$.
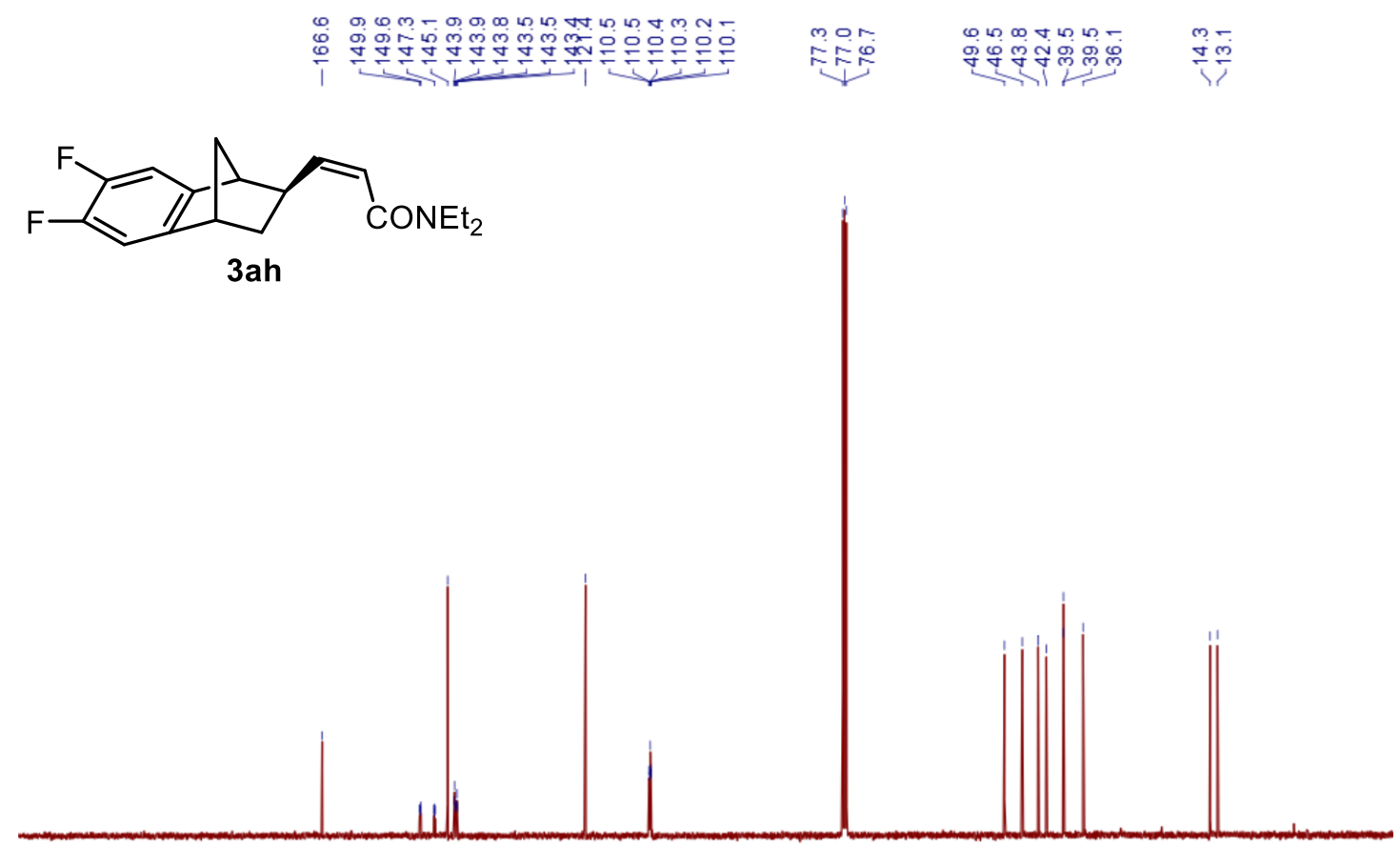

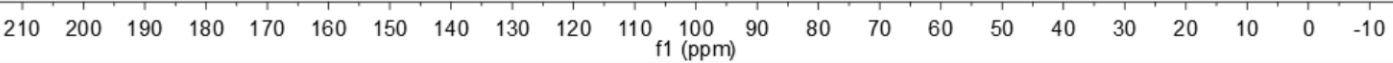

Figure S50. ${ }^{13} \mathrm{C}$ NMR $\left(101 \mathrm{MHz}, \mathrm{CDCl}_{3}\right)$ of 3ah. 


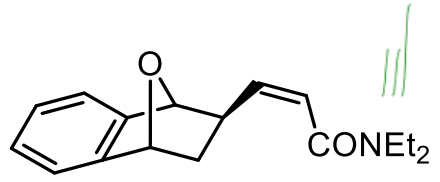

3ai

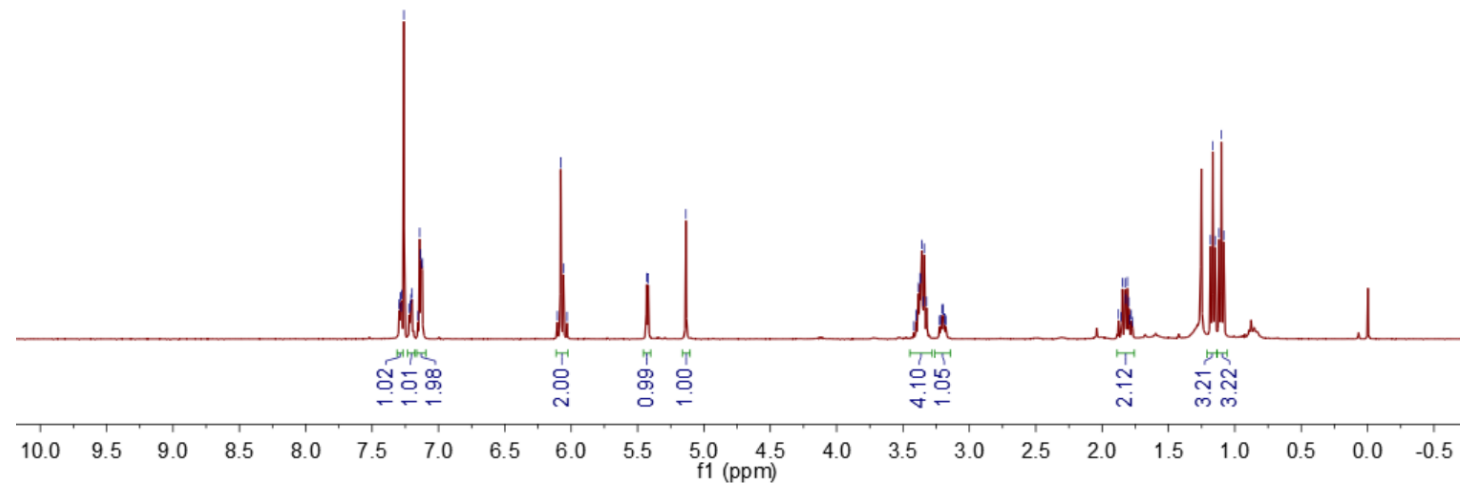

Figure S51. ${ }^{1} \mathrm{H}$ NMR $\left(400 \mathrm{MHz}, \mathrm{CDCl}_{3}\right)$ of 3ai.

\begin{tabular}{|c|c|c|c|}
\hline 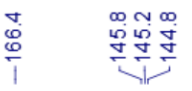 & 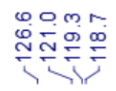 & 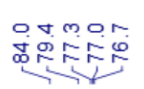 & 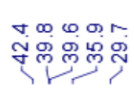 \\
\hline
\end{tabular}

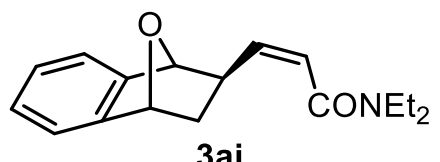

$3 a i$

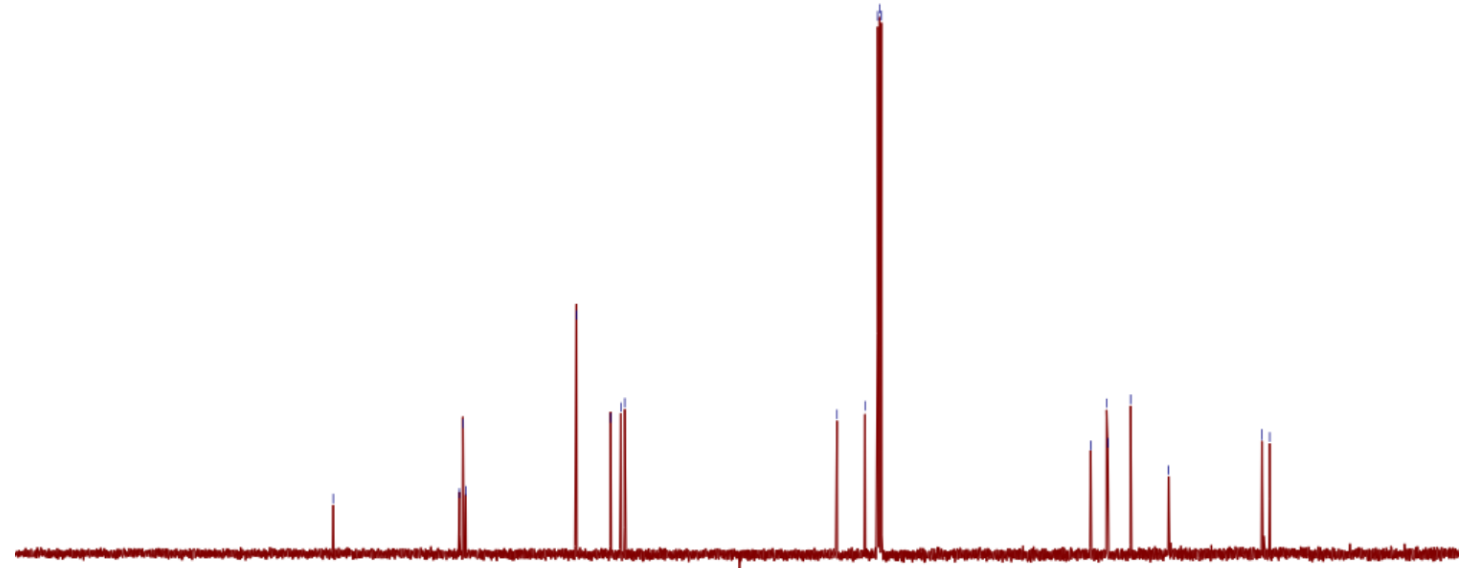

$\begin{array}{llllllllllllllllllllll}210 & 200 & 190 & 180 & 170 & 160 & 150 & 140 & 130 & 120 & 110 \begin{array}{l}100 \\ \mathrm{f} 1(\mathrm{ppm})\end{array} & 90 & 80 & 70 & 60 & 50 & 40 & 30 & 20 & 10 & 0 & -10\end{array}$

Figure S52. ${ }^{13} \mathrm{C}$ NMR $\left(101 \mathrm{MHz}, \mathrm{CDCl}_{3}\right)$ of 3ai. 


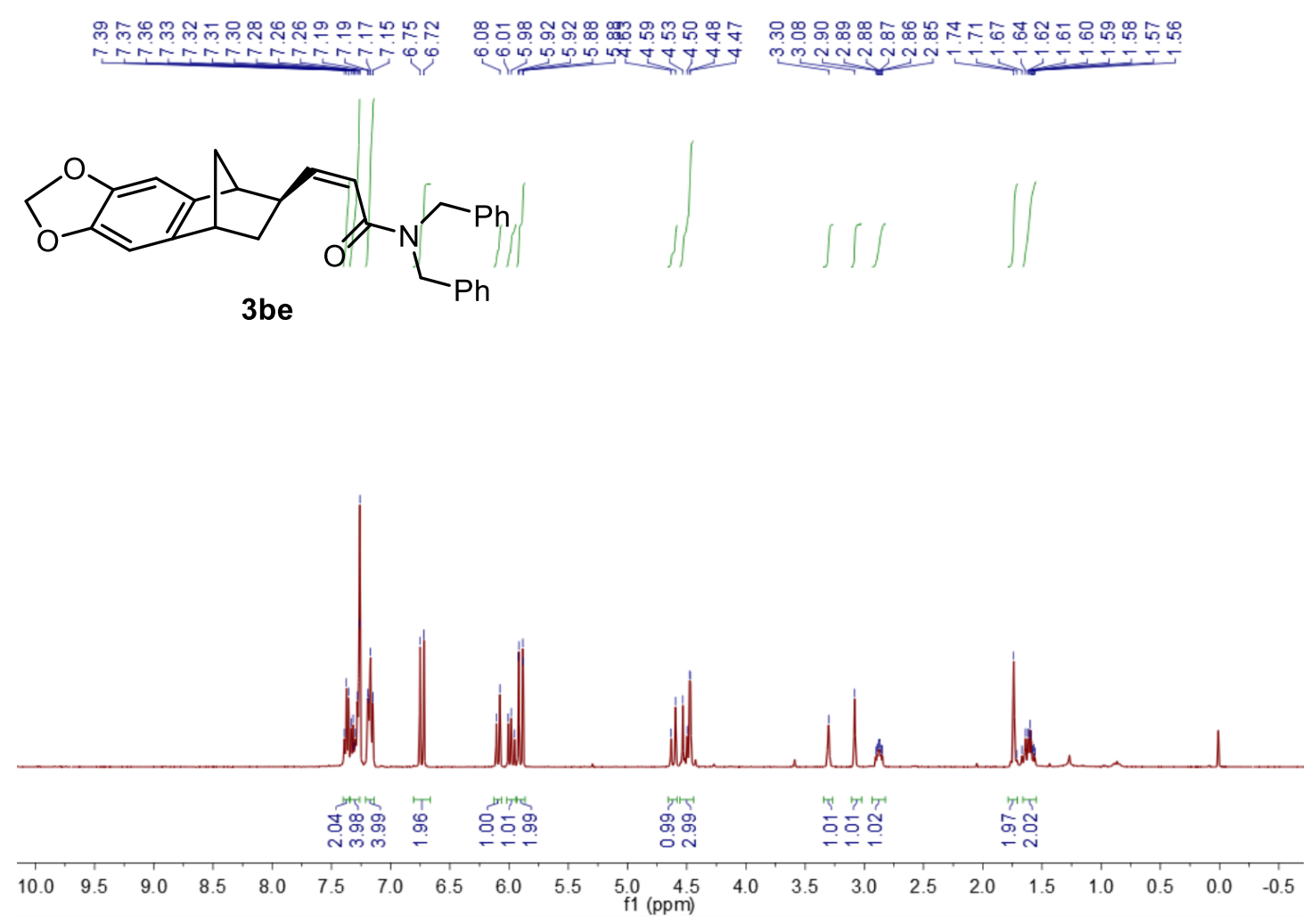

Figure S53. ${ }^{1} \mathrm{H}$ NMR $\left(400 \mathrm{MHz}, \mathrm{CDCl}_{3}\right)$ of $\mathbf{3 b e}$.

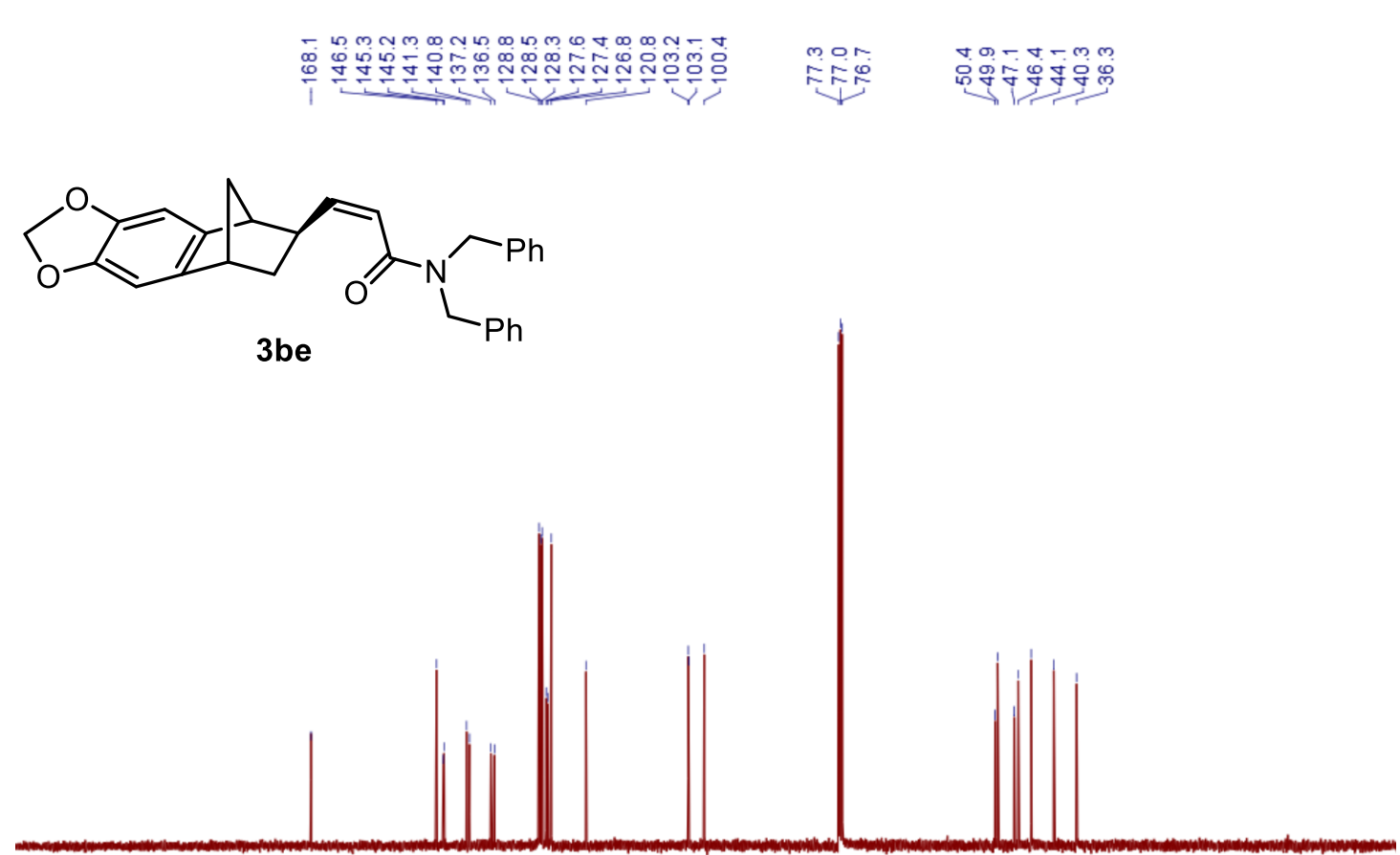

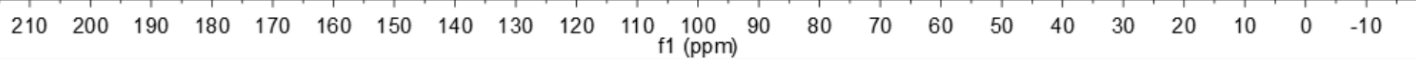

Figure S54. ${ }^{13} \mathrm{C}$ NMR $\left(101 \mathrm{MHz}, \mathrm{CDCl}_{3}\right)$ of $3 \mathbf{b e}$. 
<smiles>COCCN(CCOC)C(=O)/C=C\[C@H]1CC2CC1C1C=C3OCOC3=CC21</smiles>
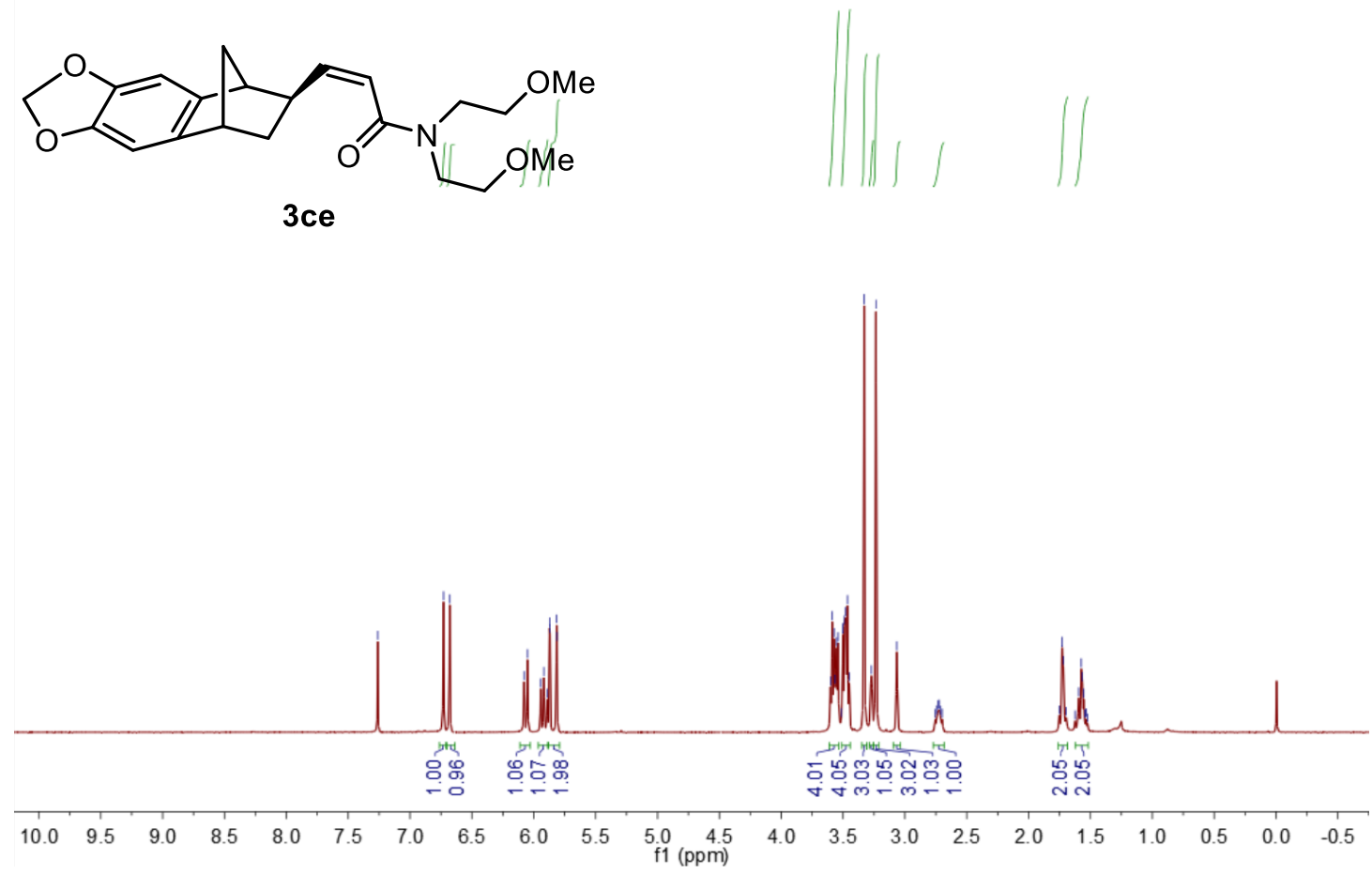

Figure S55. ${ }^{1} \mathrm{H}$ NMR $\left(400 \mathrm{MHz}, \mathrm{CDCl}_{3}\right)$ of $3 \mathbf{c e}$.

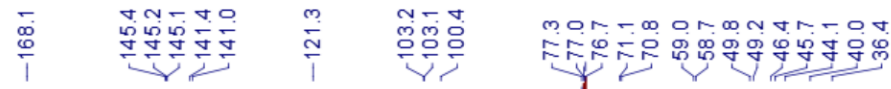<smiles>COCCN(CCOC)C(=O)/C=C\[C@H]1CC2CC1C1C=C3OCOC3=CC21</smiles>

3ce

$\begin{array}{lllllllllllllllllllllll}210 & 200 & 190 & 180 & 170 & 160 & 150 & 140 & 130 & 120 & 110 & 100 & 90 & 80 & 70 & 60 & 50 & 40 & 30 & 20 & 10 & 0 & -10\end{array}$

Figure S56. ${ }^{13} \mathrm{C}$ NMR $\left(101 \mathrm{MHz}, \mathrm{CDCl}_{3}\right)$ of 3ce. 


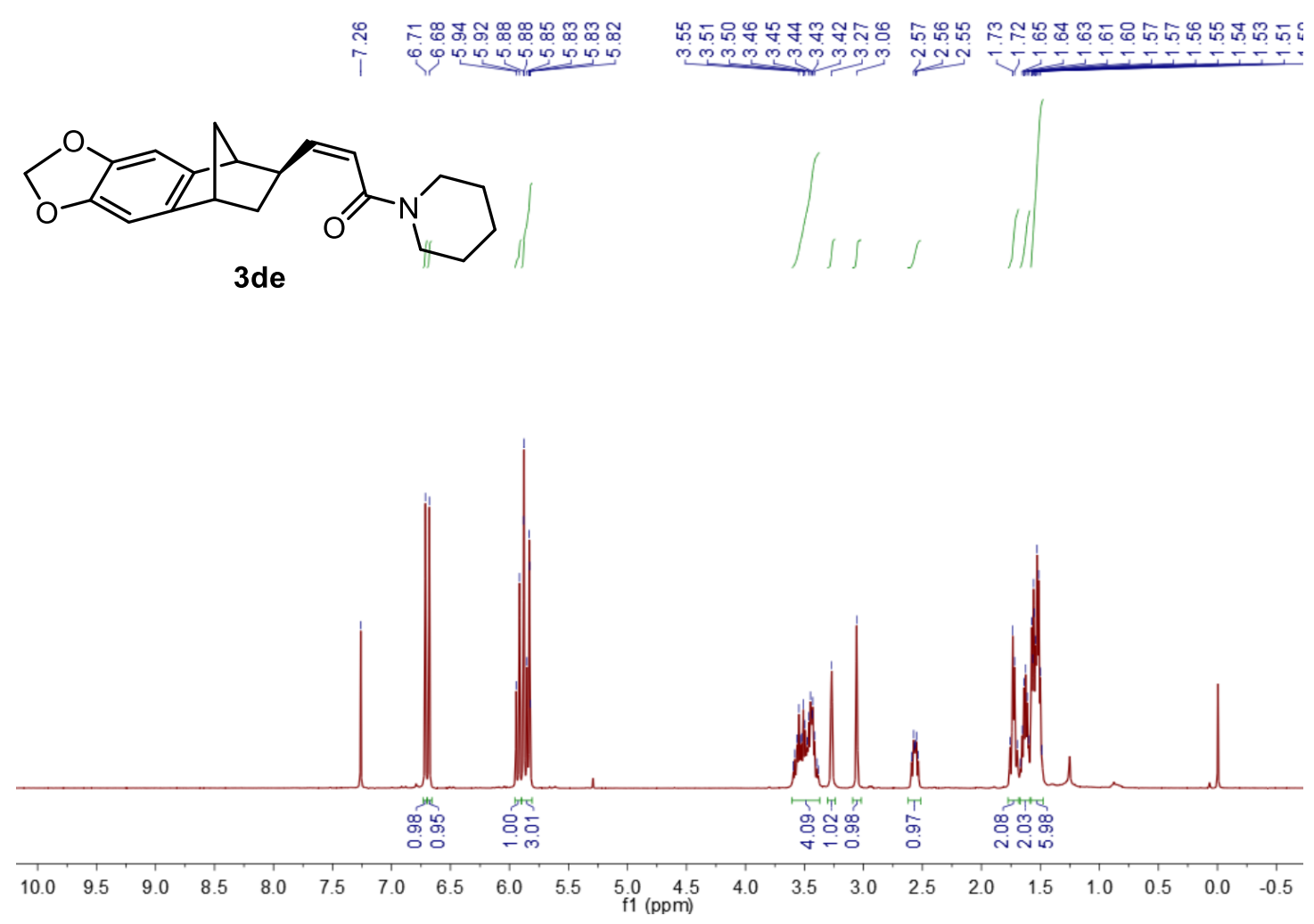

Figure S57. ${ }^{1} \mathrm{H} \mathrm{NMR}\left(400 \mathrm{MHz}, \mathrm{CDCl}_{3}\right)$ of $\mathbf{3 d e}$.

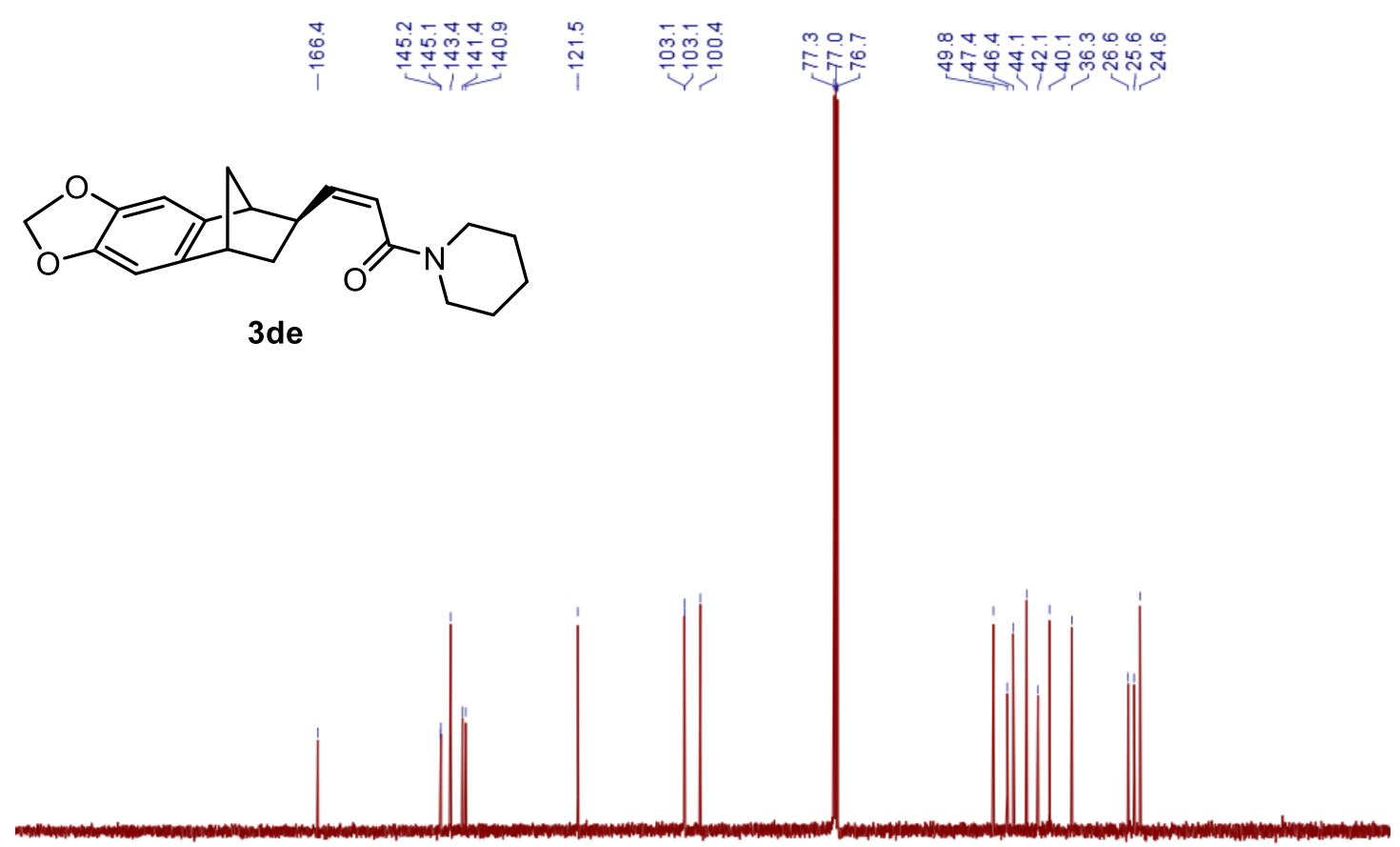

$\begin{array}{lllllllllllllllllllllll}210 & 200 & 190 & 180 & 170 & 160 & 150 & 140 & 130 & 120 & 110 & 100 & 90 & 80 & 70 & 60 & 50 & 40 & 30 & 20 & 10 & 0 & -10\end{array}$

Figure S58. ${ }^{13} \mathrm{C}$ NMR $\left(101 \mathrm{MHz}, \mathrm{CDCl}_{3}\right)$ of 3de. 


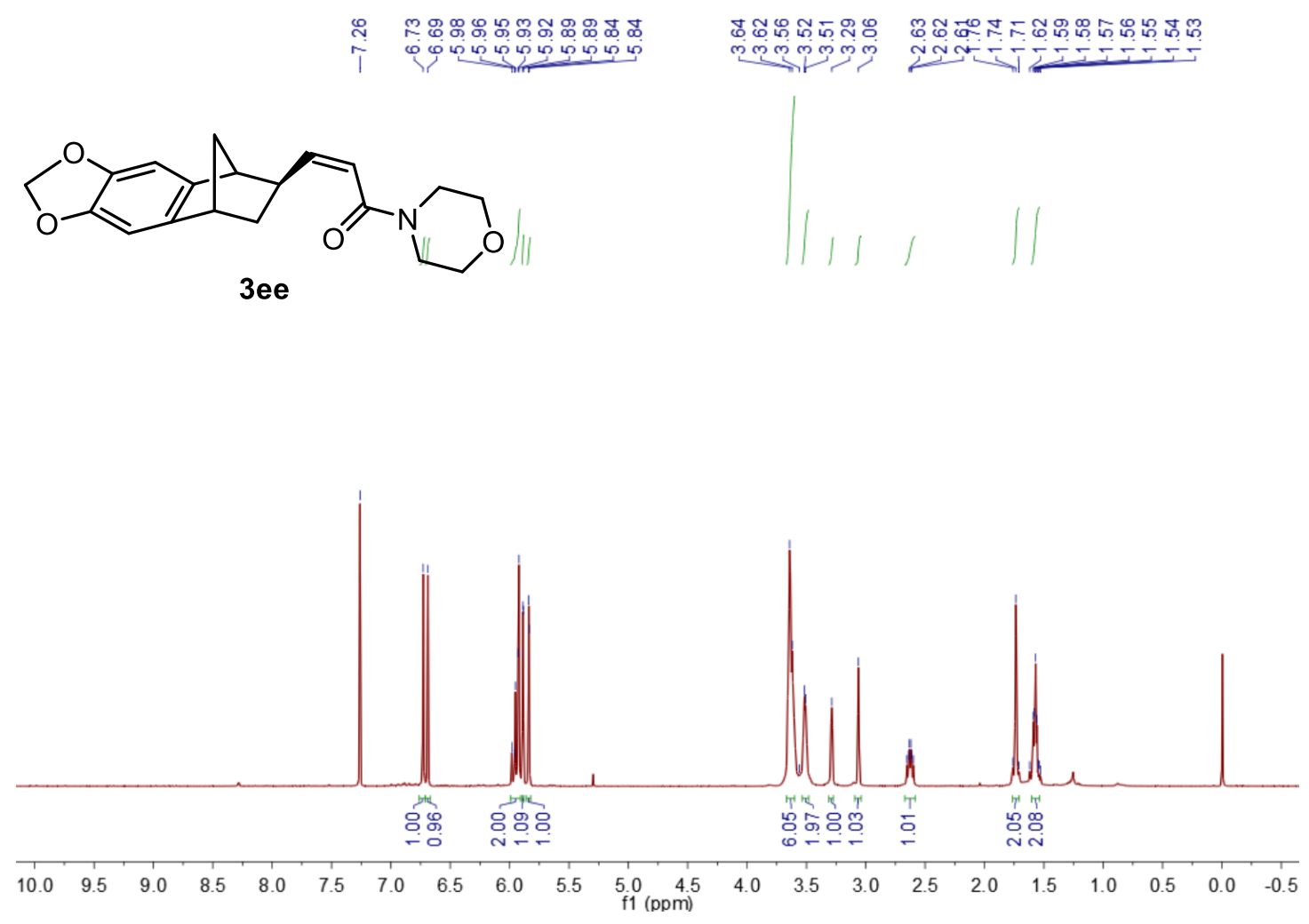

Figure S59. ${ }^{1} \mathrm{H} \mathrm{NMR}\left(400 \mathrm{MHz}, \mathrm{CDCl}_{3}\right)$ of $\mathbf{3 e e}$.
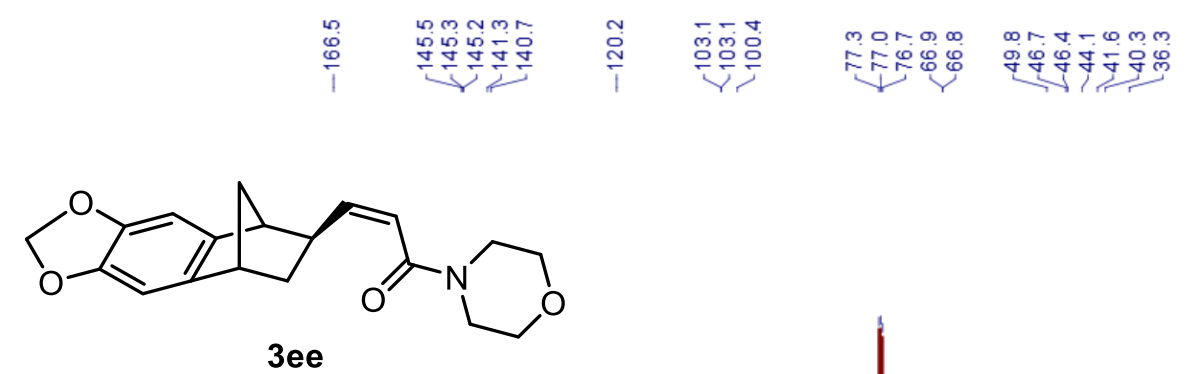

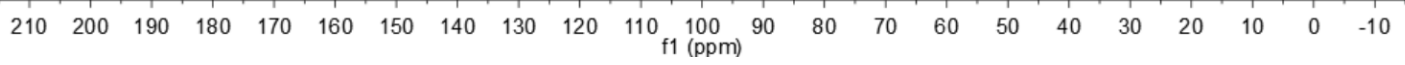

Figure S60. ${ }^{13} \mathrm{C}$ NMR $\left(101 \mathrm{MHz}, \mathrm{CDCl}_{3}\right)$ of 3ee. 

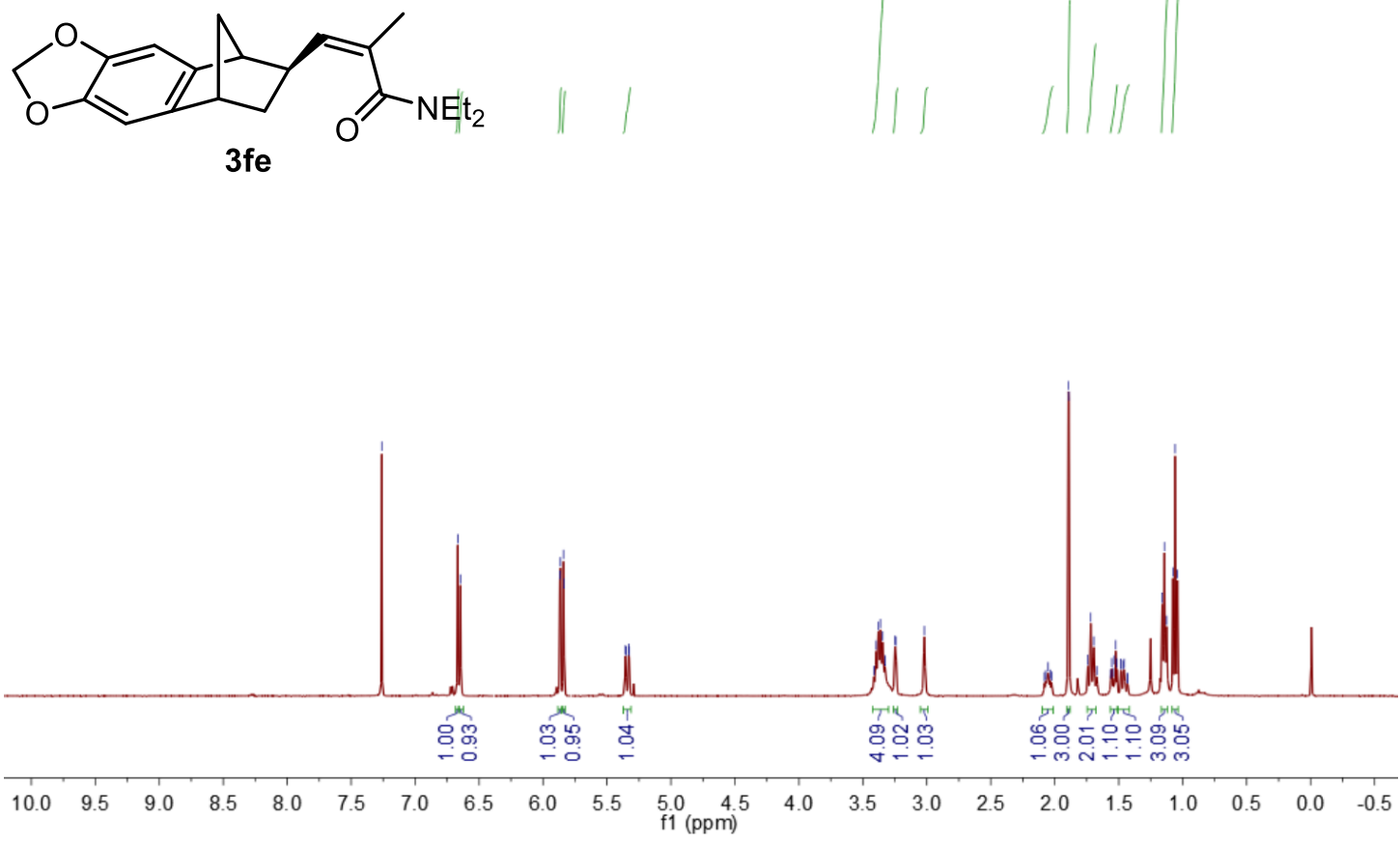

Figure S61. ${ }^{1} \mathrm{H}$ NMR (400 MHz, $\mathrm{CDCl}_{3}$ ) of $\mathbf{3 f e}$.

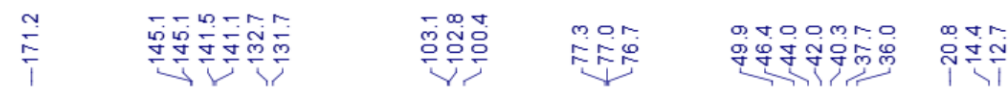<smiles>CCNC(=O)/C(C)=C\[C@H]1C[C@@H]2CC3c4cc5c(cc4C3C1C2)OCO5</smiles>

$3 f e$

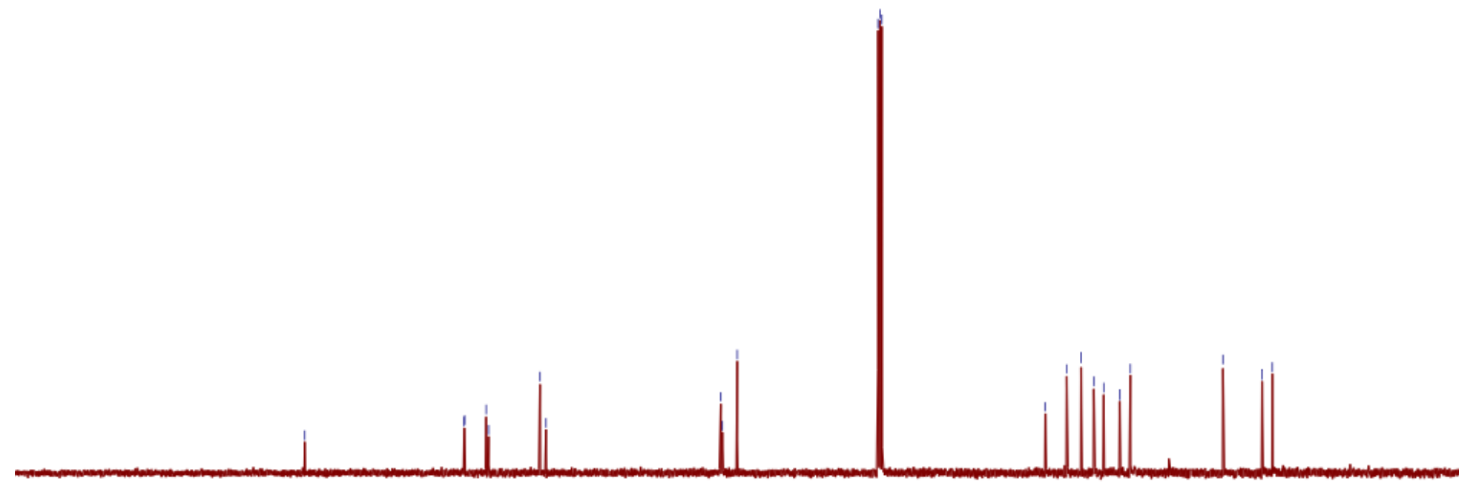

$\begin{array}{llllllllllllllllllllll}210 & 200 & 190 & 180 & 170 & 160 & 150 & 140 & 130 & 120 & 110 \begin{array}{l}100 \\ \mathrm{f} 1(\mathrm{ppm})\end{array} & 90 & 80 & 70 & 60 & 50 & 40 & 30 & 20 & 10 & 0 & -10\end{array}$

Figure S62. ${ }^{13} \mathrm{C}$ NMR $\left(101 \mathrm{MHz}, \mathrm{CDCl}_{3}\right)$ of $\mathbf{3 f e}$. 
<smiles>CCCCNC(=O)/C=C\[C@H]1C[C@H]2CC1C1C=C3OCOC3=CC12</smiles>
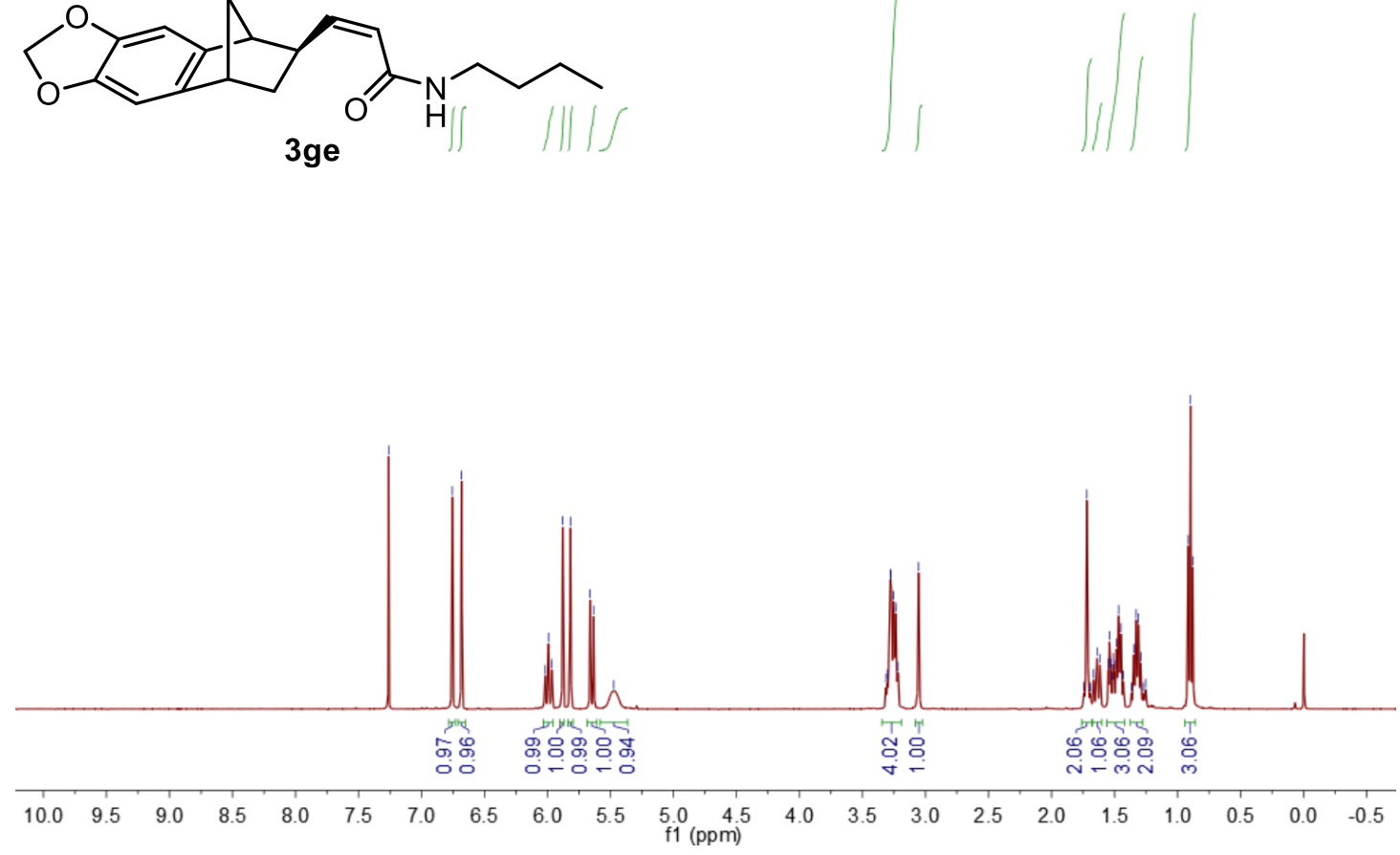

Figure S63. ${ }^{1} \mathrm{H} \mathrm{NMR}\left(400 \mathrm{MHz}, \mathrm{CDCl}_{3}\right)$ of 3ge.

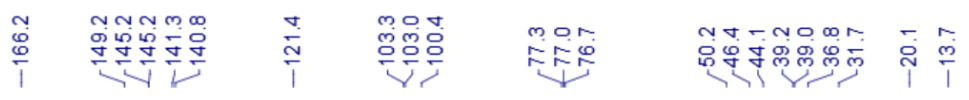<smiles>CCCCNC(=O)/C=C\[C@H]1CC2CC1C1C=C3OCOC3=CC21</smiles>

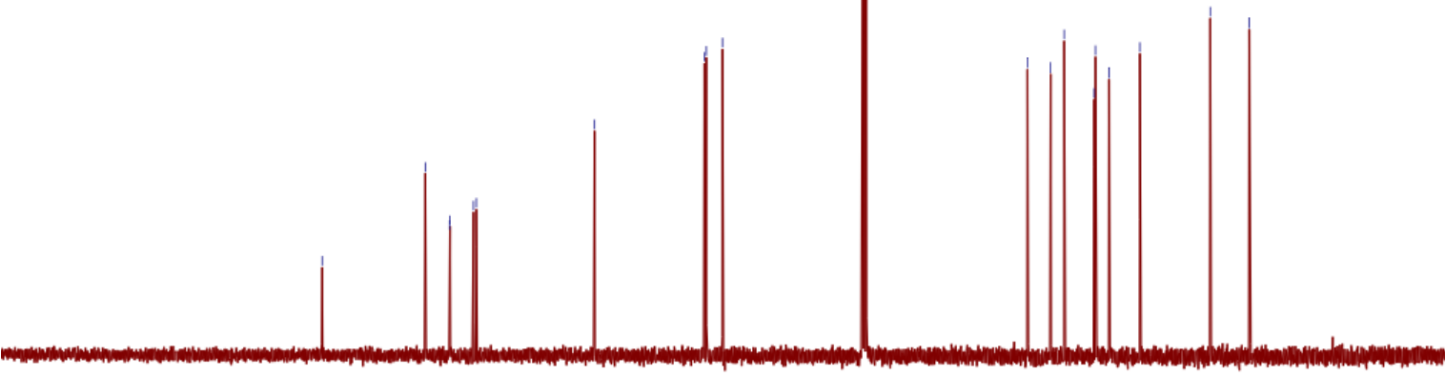

$\begin{array}{lllllllllllllllllllllll}210 & 200 & 190 & 180 & 170 & 160 & 150 & 140 & 130 & 120 & 110 & 100 & 100 & 80 & 70 & 60 & 50 & 40 & 30 & 20 & 10 & 0 & -10\end{array}$

Figure S64. ${ }^{13} \mathrm{C}$ NMR $\left(101 \mathrm{MHz}, \mathrm{CDCl}_{3}\right)$ of 3ge. 
ஸุ.

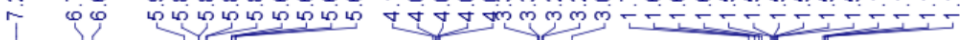<smiles>[Te][Te]</smiles>

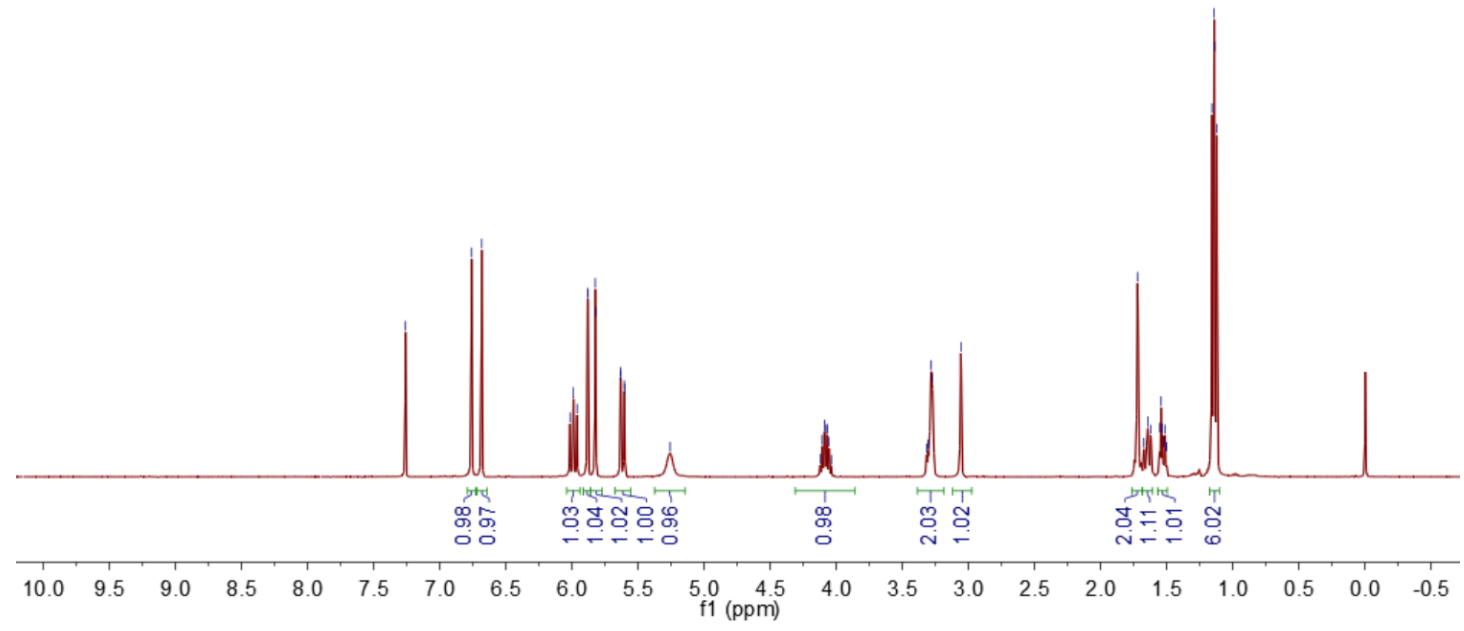

Figure $\mathbf{S 6 5} .{ }^{1} \mathrm{H}$ NMR $\left(400 \mathrm{MHz}, \mathrm{CDCl}_{3}\right)$ of 3 he.

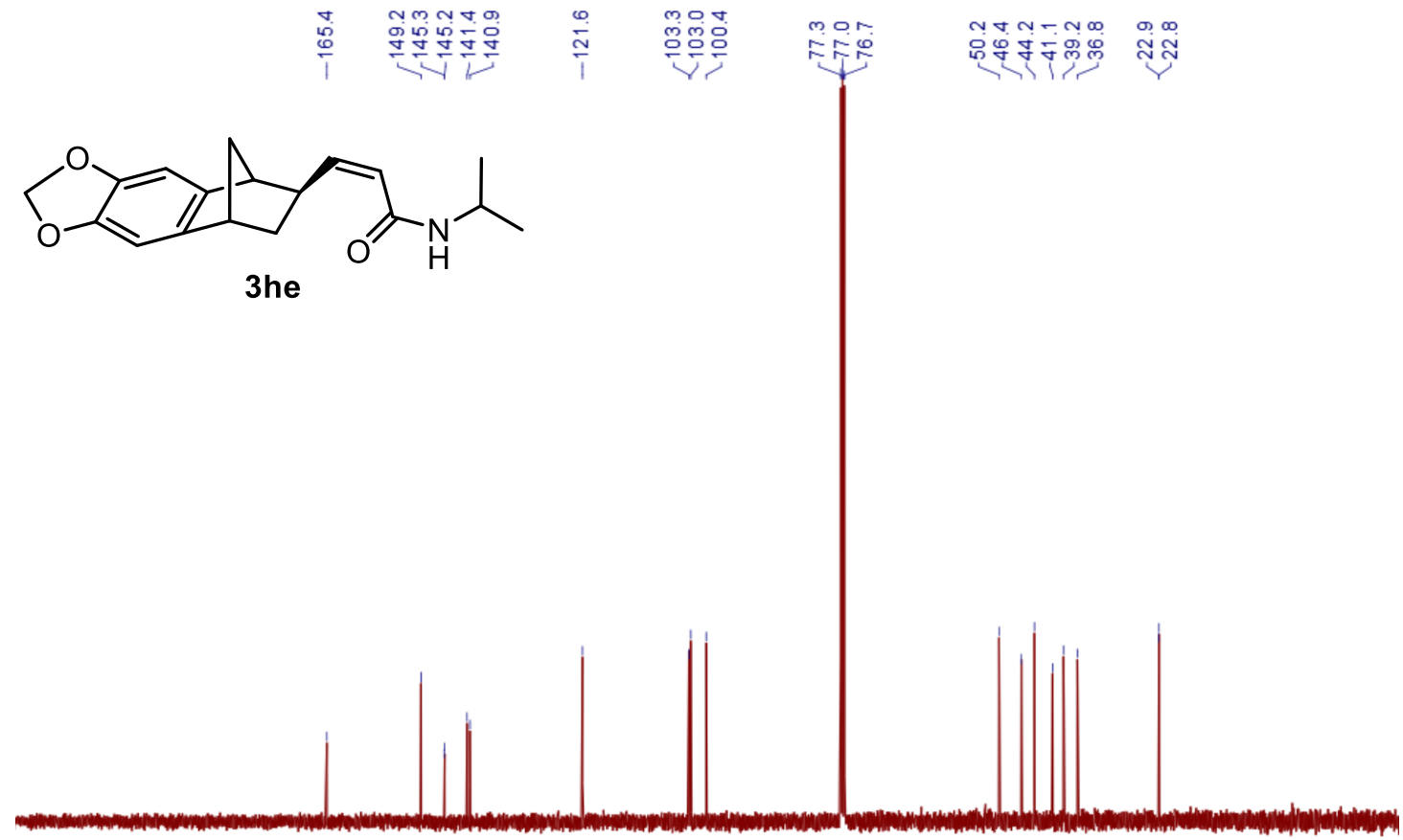

$\begin{array}{lllllllllllllllllllllll}210 & 200 & 190 & 180 & 170 & 160 & 150 & 140 & 130 & 120 & 110 \underset{\mathrm{f} 1}{(\mathrm{ppm})} \mathbf{( 0 0} & 90 & 80 & 70 & 60 & 50 & 40 & 30 & 20 & 10 & 0 & -10\end{array}$

Figure S66. ${ }^{13} \mathrm{C}$ NMR $\left(101 \mathrm{MHz}, \mathrm{CDCl}_{3}\right)$ of 3 he. 

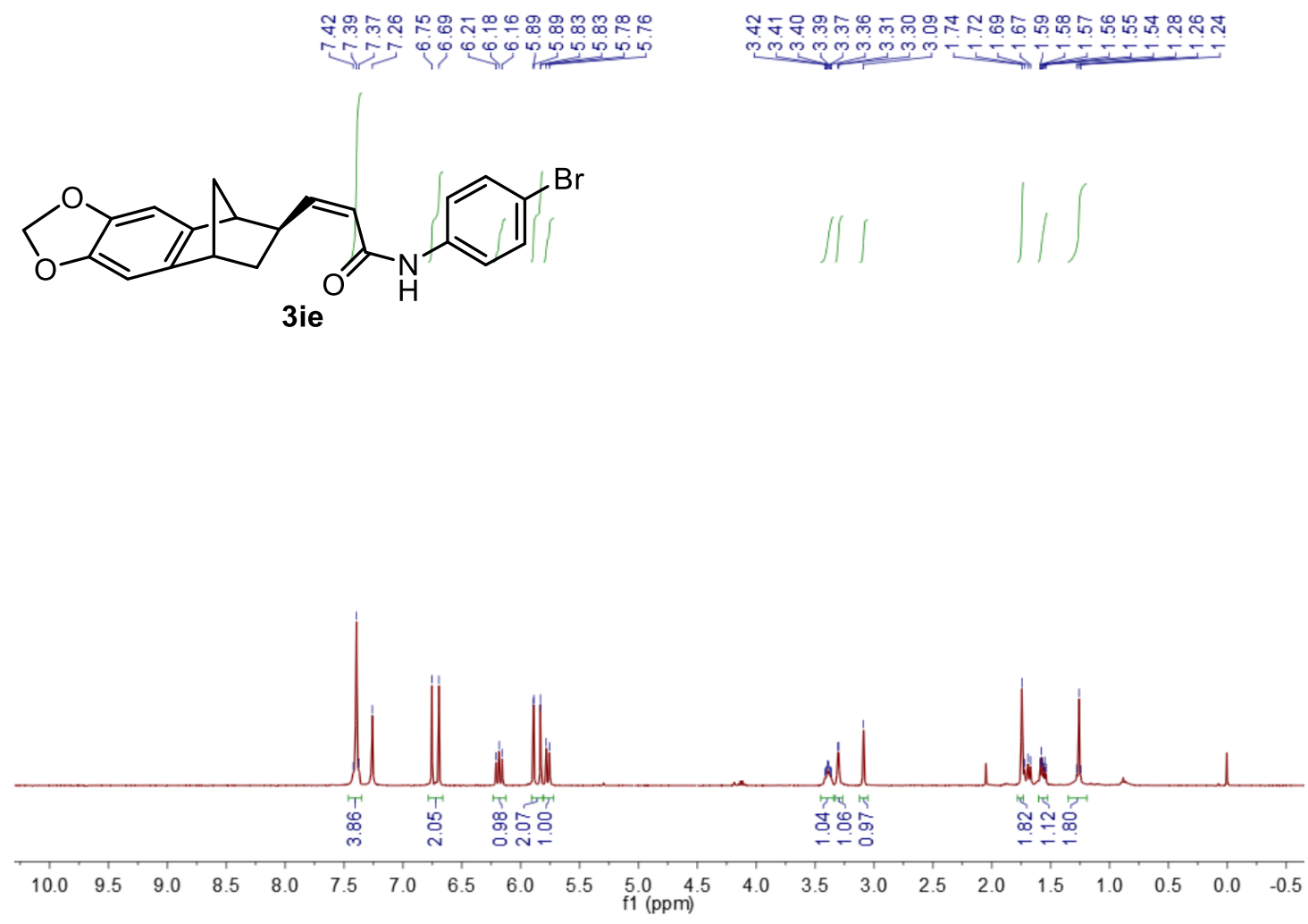

Figure S67. ${ }^{1} \mathrm{H}$ NMR $\left(400 \mathrm{MHz}, \mathrm{CDCl}_{3}\right)$ of 3ie.

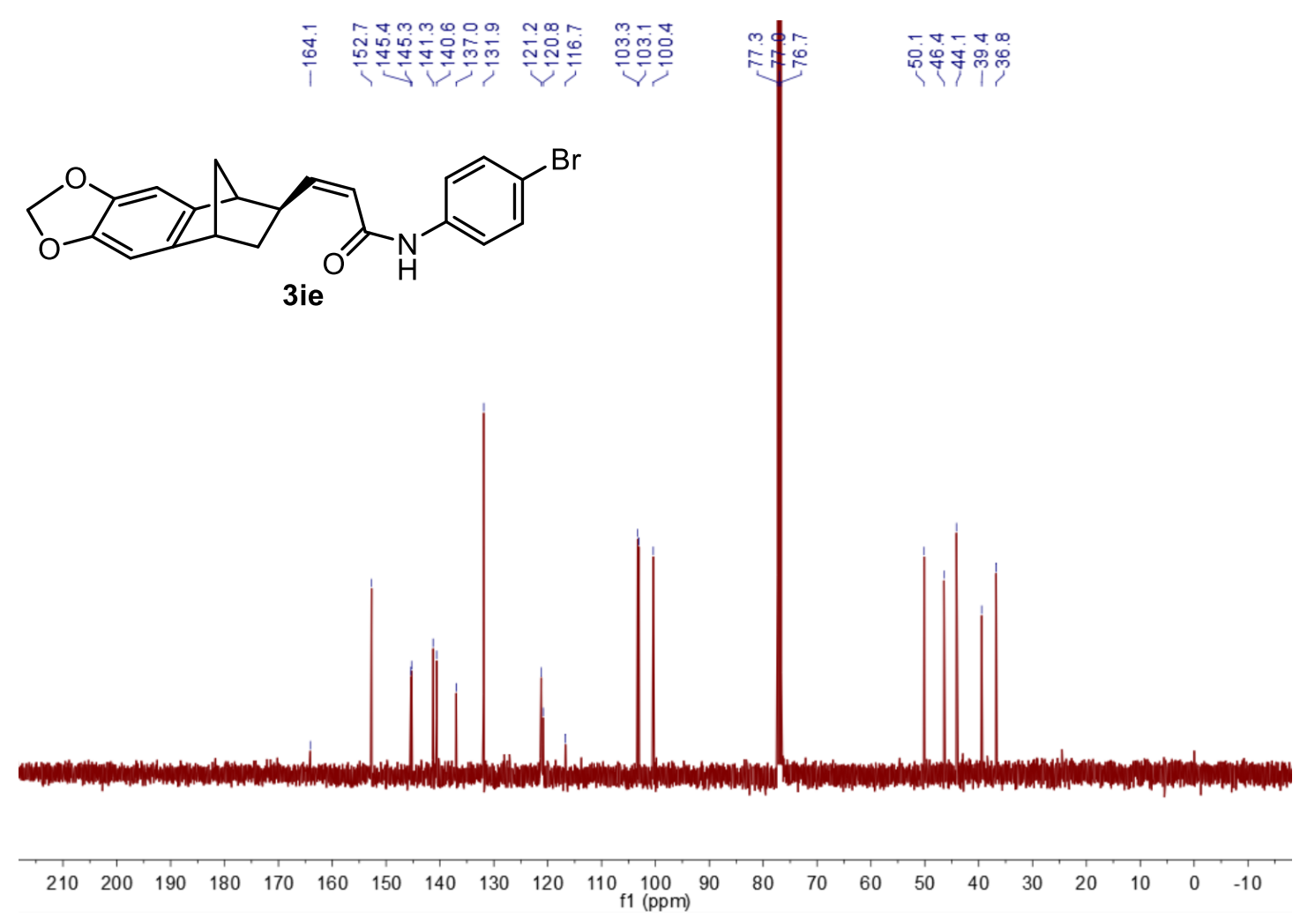

Figure S68. ${ }^{13} \mathrm{C}$ NMR $\left(101 \mathrm{MHz}, \mathrm{CDCl}_{3}\right)$ of 3ie. 


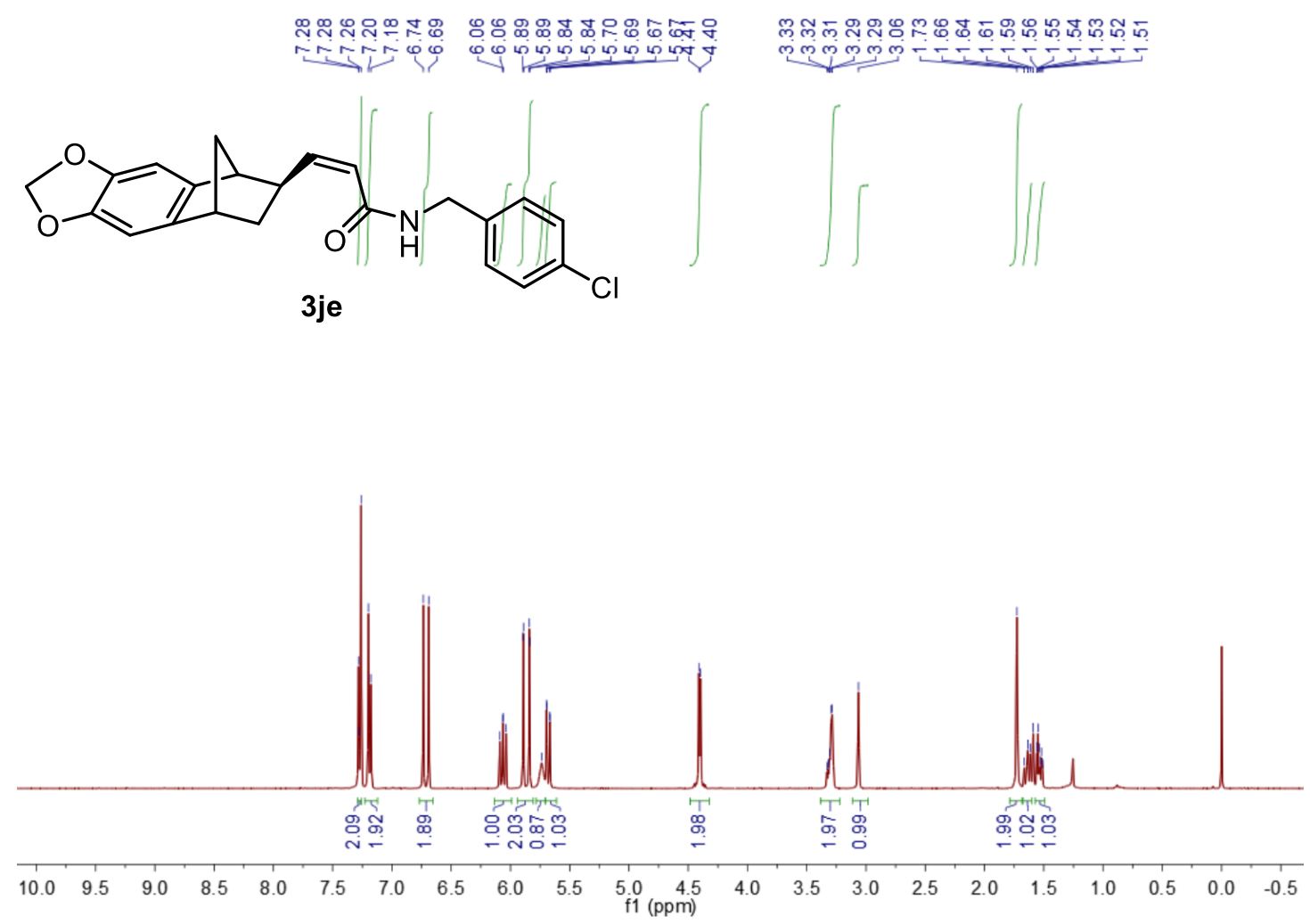

Figure S69. ${ }^{1} \mathrm{H} \mathrm{NMR}\left(400 \mathrm{MHz}, \mathrm{CDCl}_{3}\right)$ of $\mathbf{3 j e}$.

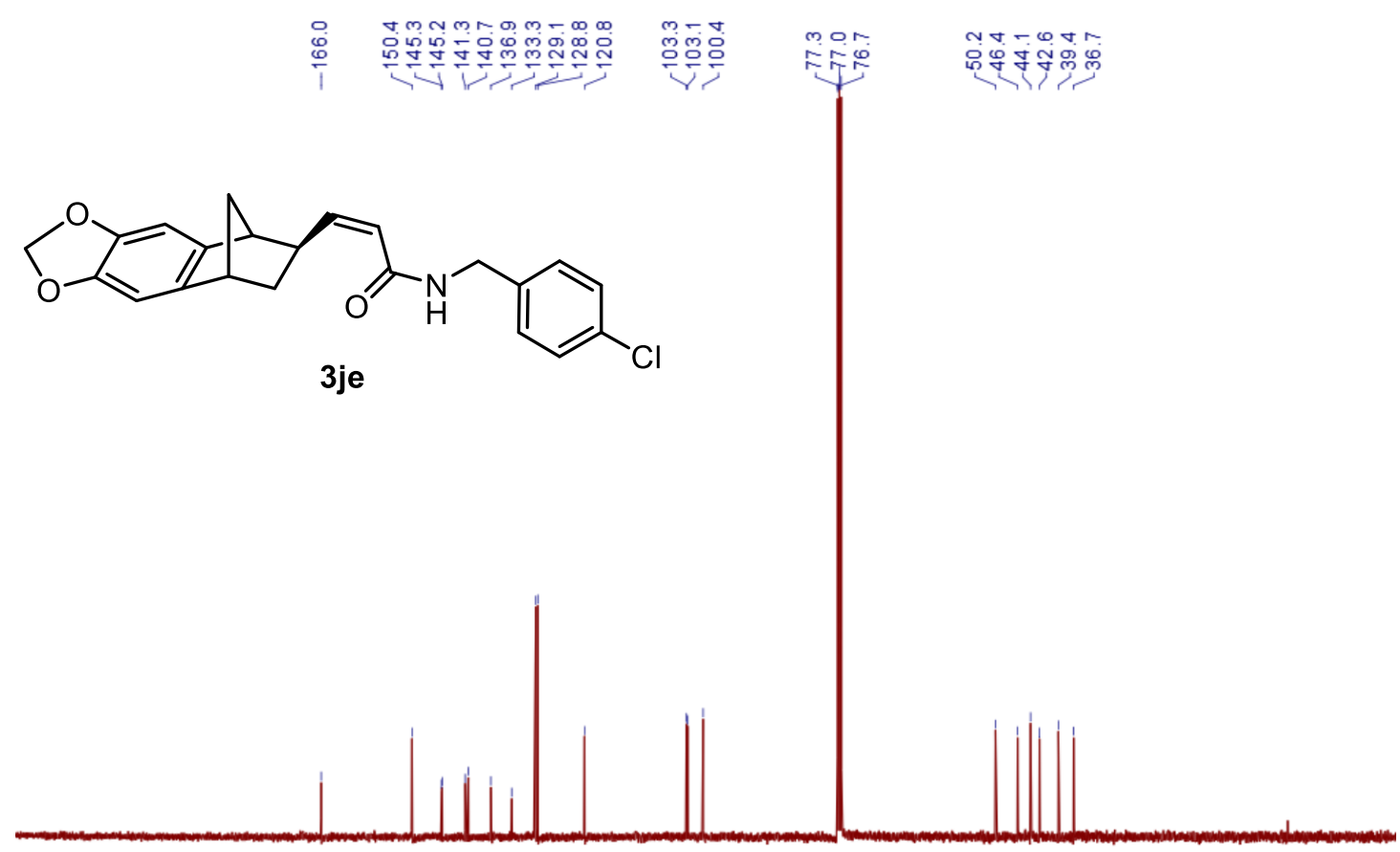

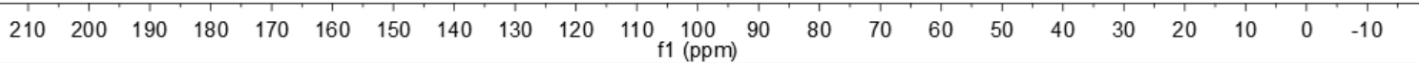

Figure S70. ${ }^{13} \mathrm{C} \mathrm{NMR}\left(101 \mathrm{MHz}, \mathrm{CDCl}_{3}\right)$ of $\mathbf{3 j e}$. 


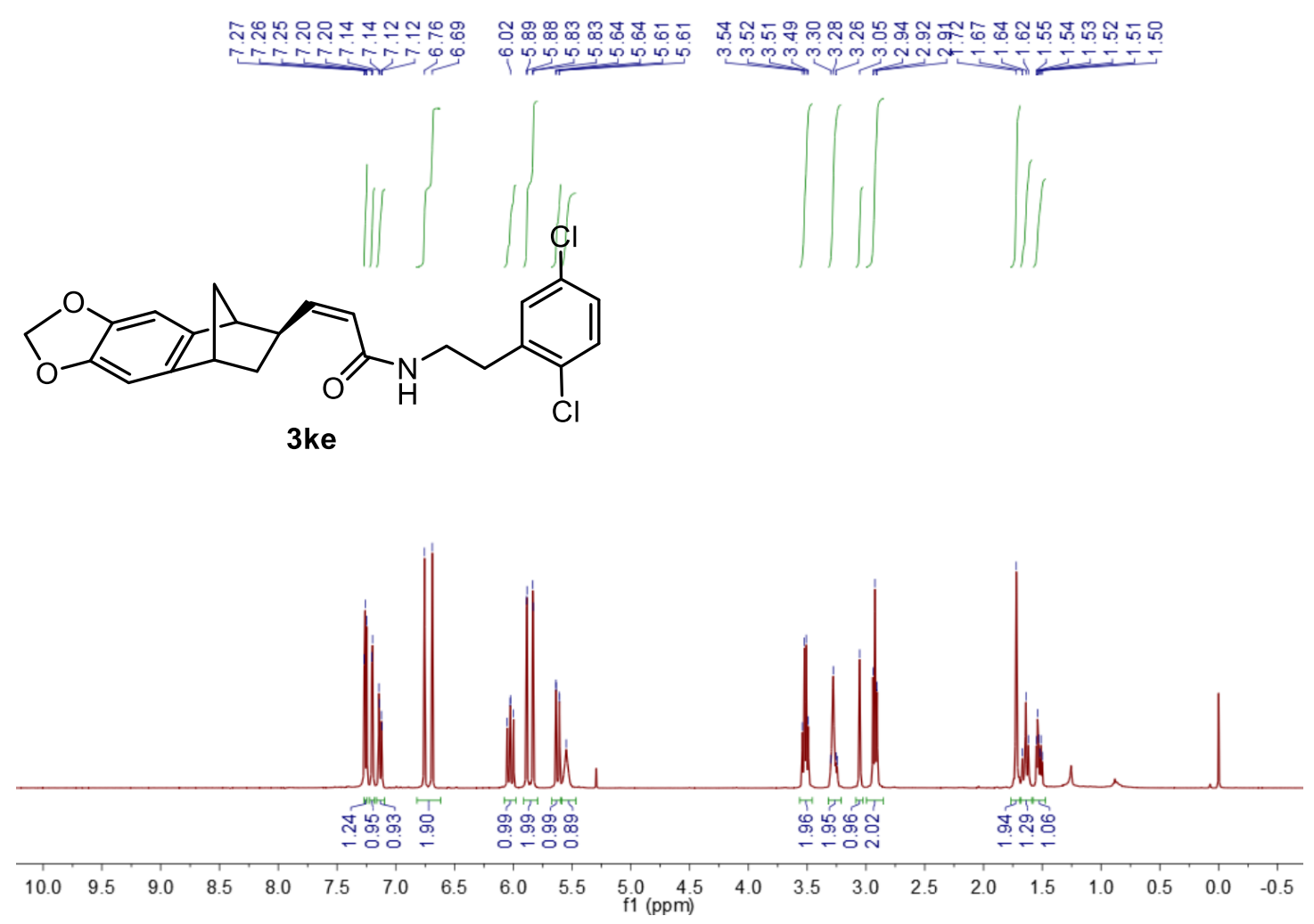

Figure S71. ${ }^{1} \mathrm{H}$ NMR $\left(400 \mathrm{MHz}, \mathrm{CDCl}_{3}\right)$ of 3 ke.

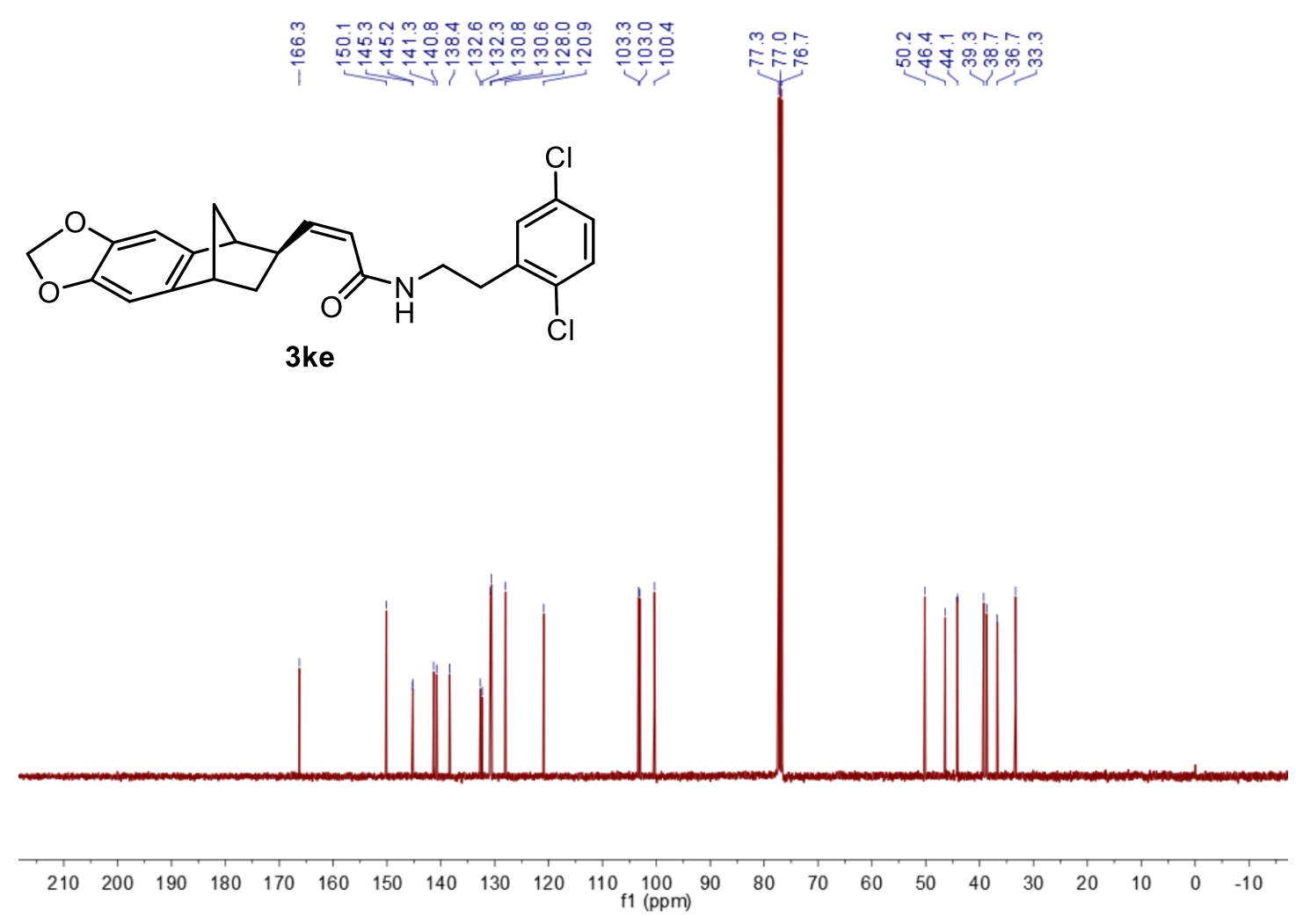

Figure S72. ${ }^{13} \mathrm{C}$ NMR $\left(101 \mathrm{MHz}, \mathrm{CDCl}_{3}\right)$ of 3 ke. 


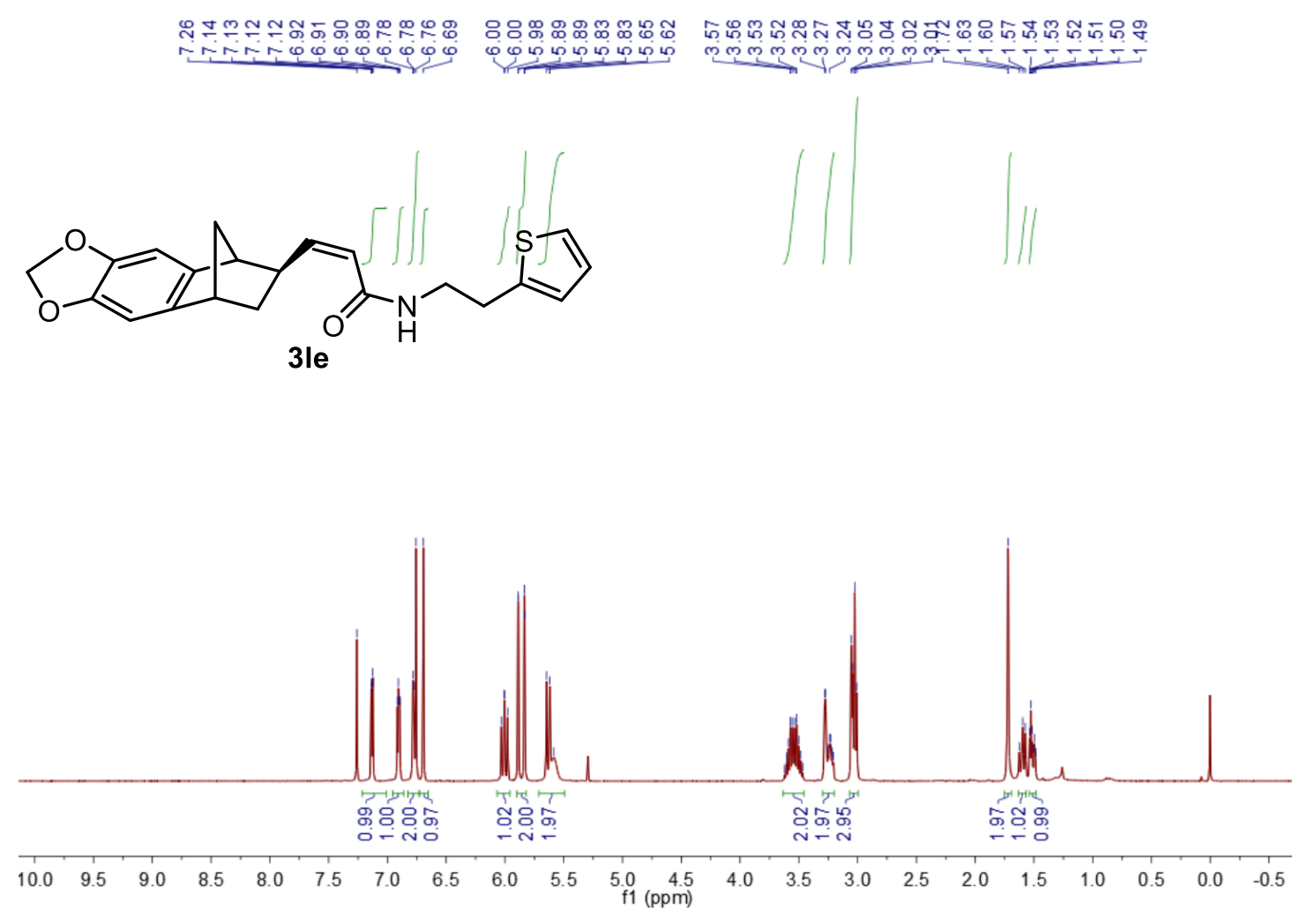

Figure S73. ${ }^{1} \mathrm{H}$ NMR (400 MHz, $\left.\mathrm{CDCl}_{3}\right)$ of 3le.
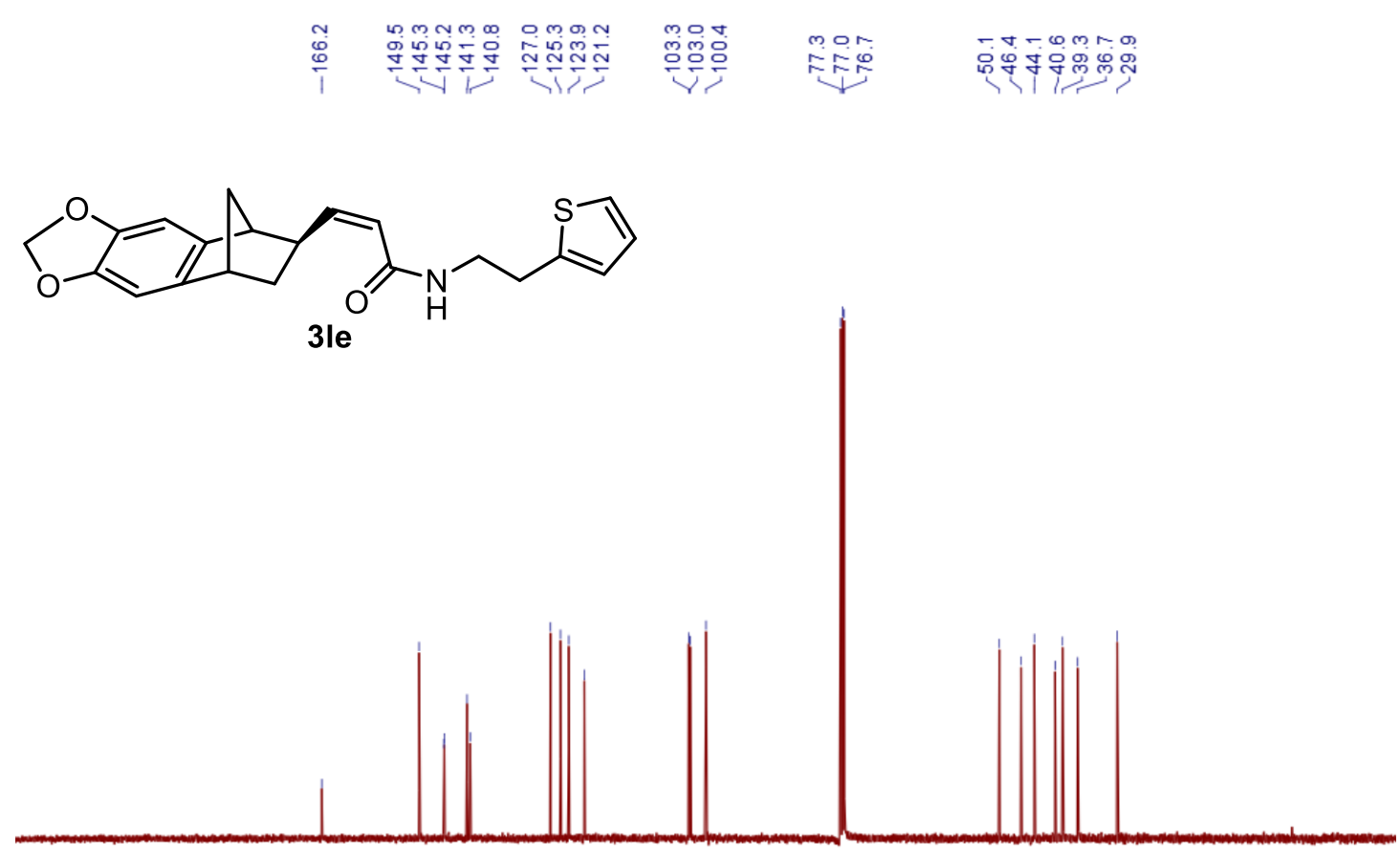

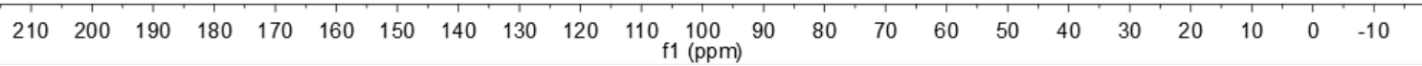

Figure S74. ${ }^{13} \mathrm{C}$ NMR (101 MHz, $\left.\mathrm{CDCl}_{3}\right)$ of 3le. 
<smiles>CCCCOC(=O)/C=C\C1CC2CCC1C2</smiles>
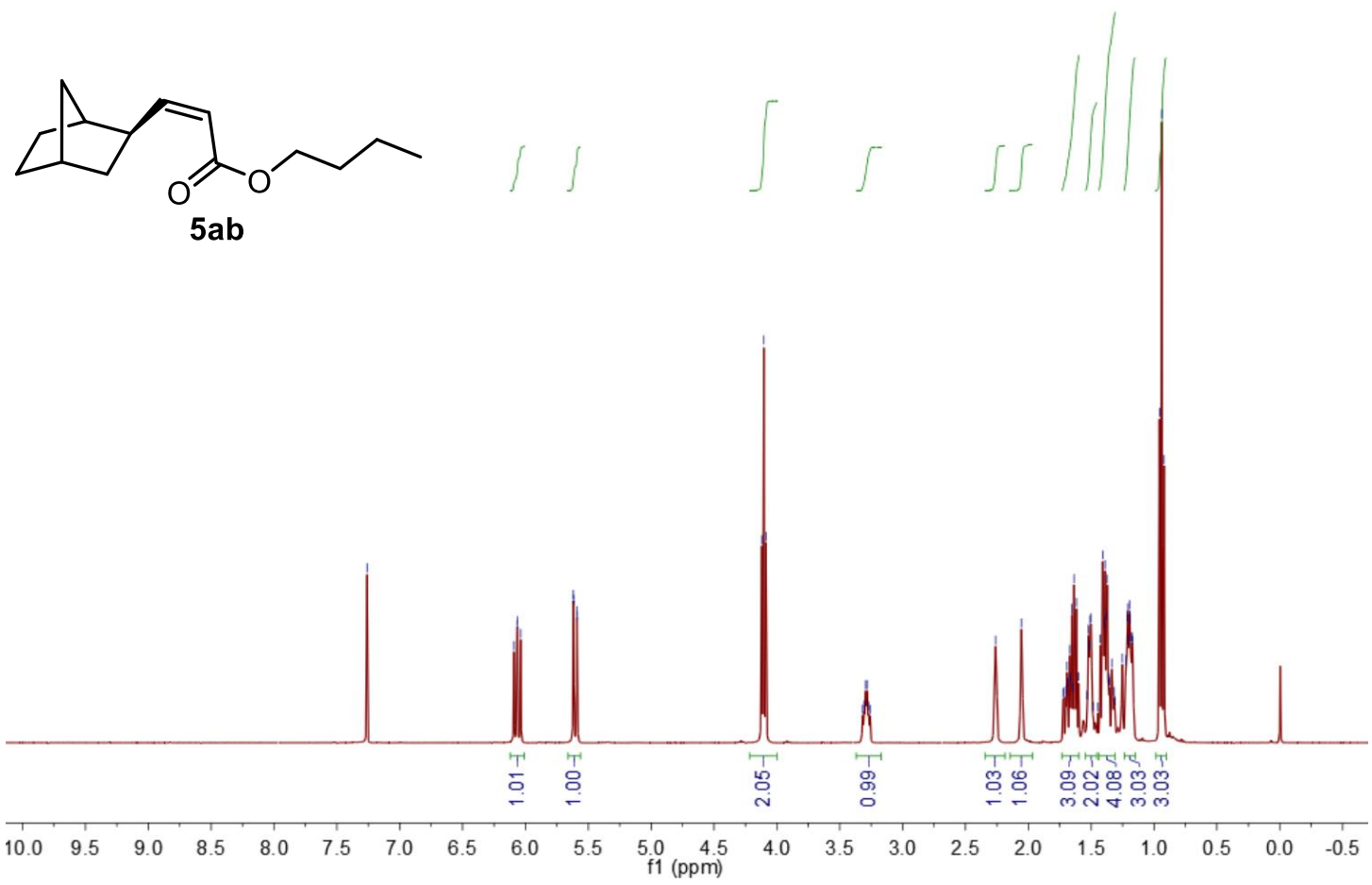

Figure S75. ${ }^{1} \mathrm{H} \mathrm{NMR}\left(400 \mathrm{MHz}, \mathrm{CDCl}_{3}\right)$ of $\mathbf{5 a b}$.

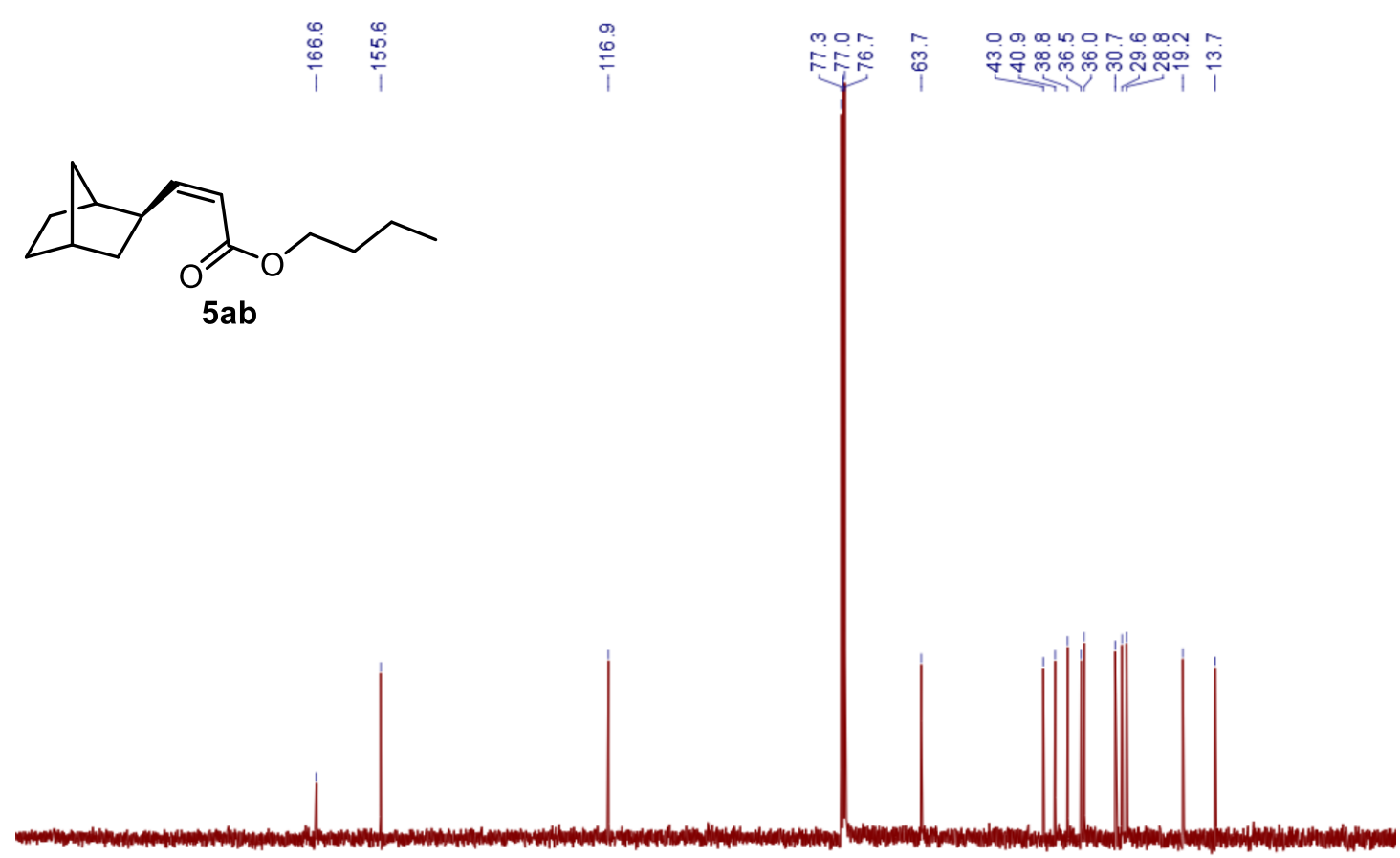

$\begin{array}{llllllllllllllllllllll}210 & 200 & 190 & 180 & 170 & 160 & 150 & 140 & 130 & 120 & 110 \begin{array}{l}100 \\ \mathrm{f} 1(\mathrm{ppm})\end{array} & 90 & 80 & 70 & 60 & 50 & 40 & 30 & 20 & 10 & 0 & -10\end{array}$

Figure S76. ${ }^{13} \mathrm{C}$ NMR $\left(101 \mathrm{MHz}, \mathrm{CDCl}_{3}\right)$ of $\mathbf{5 a b}$. 

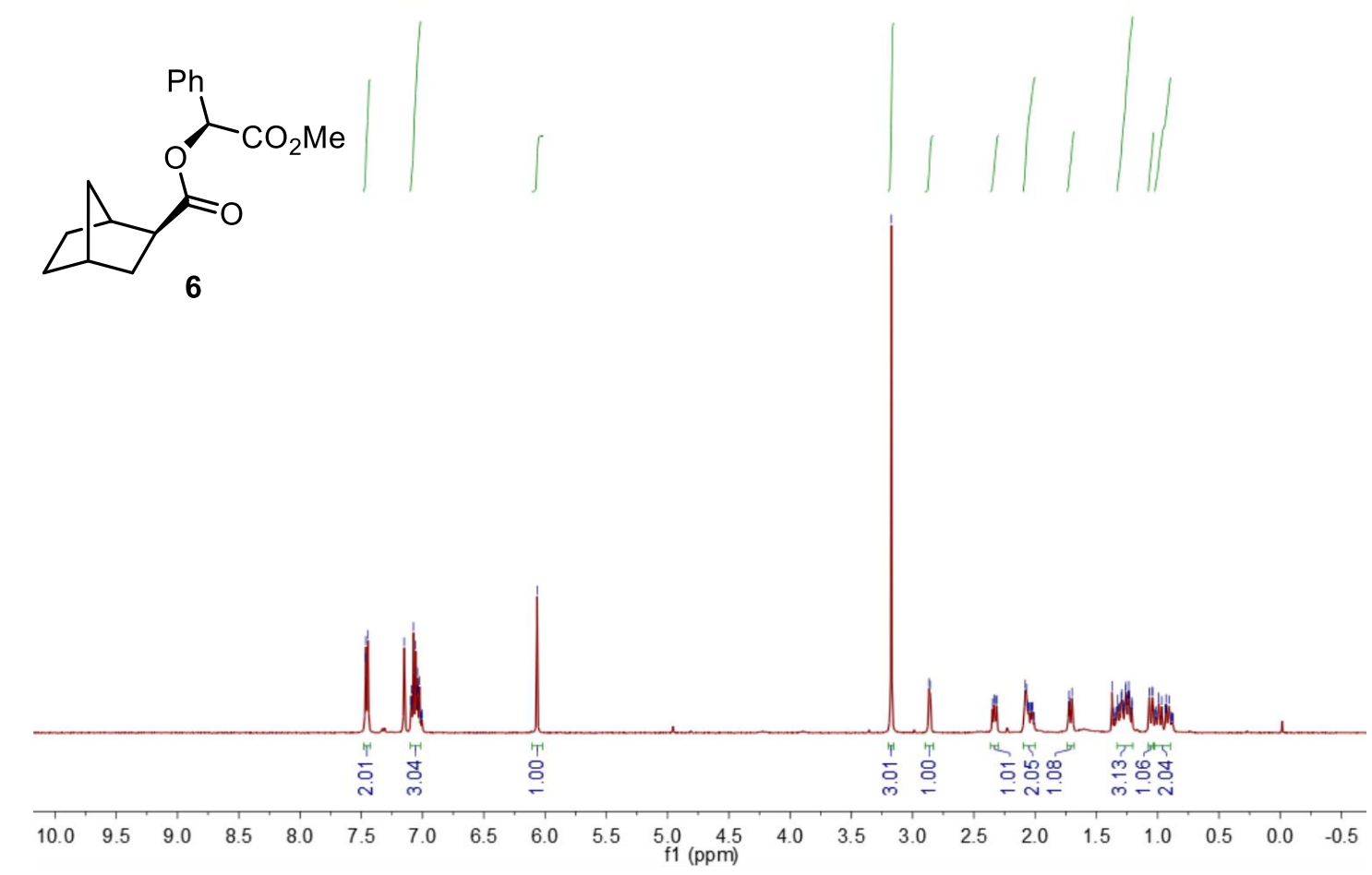

Figure S77. ${ }^{1} \mathrm{H}$ NMR $\left(400 \mathrm{MHz}, \mathrm{C}_{6} \mathrm{D}_{6}\right)$ of 6.
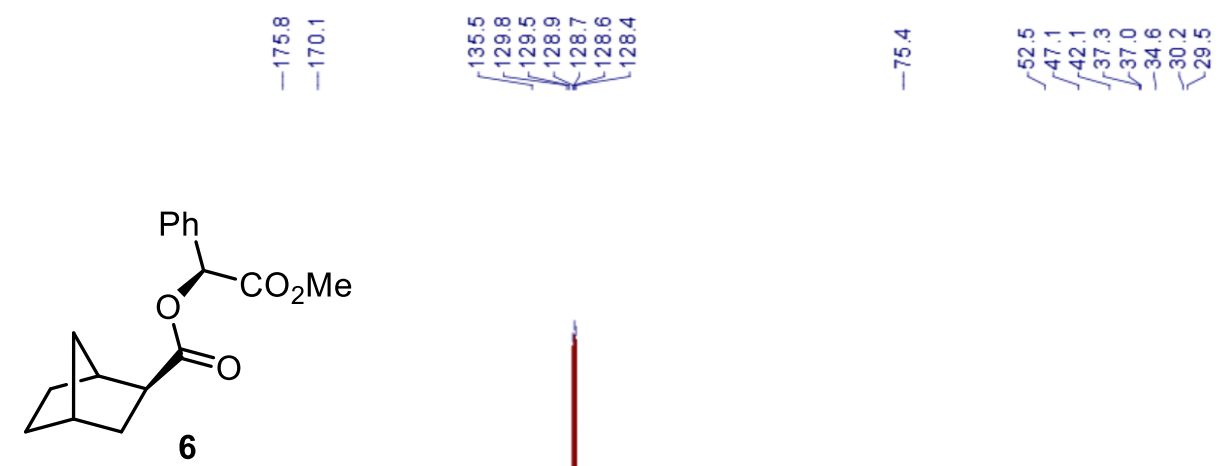

$\begin{array}{lllllllllllllllllllllllllllll}0 & 210 & 200 & 190 & 180 & 170 & 160 & 150 & 140 & 130 & 120 & 110 \begin{array}{l}100 \\ \mathrm{f} 1(\mathrm{ppm})\end{array} & 90 & 80 & 70 & 60 & 50 & 40 & 30 & 20 & 10 & 0 & -10\end{array}$

Figure S78. ${ }^{13} \mathrm{C}$ NMR $\left(101 \mathrm{MHz}, \mathrm{C}_{6} \mathrm{D}_{6}\right)$ of 6 . 


\section{Copies of HPLC spectra}

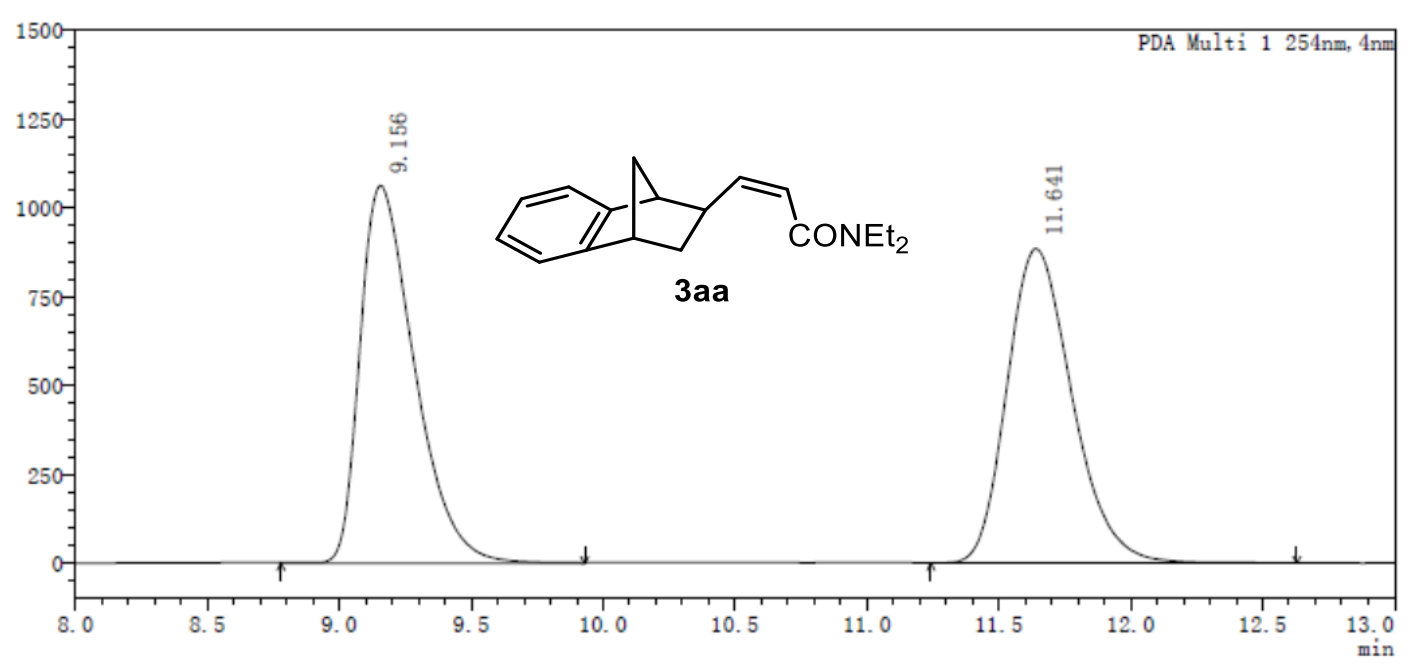

PDA Ch1 254nm

\begin{tabular}{|r|r|r|r|r|r|}
\hline 峰号 & 保留时间 & 峰宽 (高度 50\%) & 高度 & \multicolumn{1}{c|}{ 面积 } & 面积\% \\
\hline 1 & 9.156 & 0.220 & 1063511 & 15375259 & 50.227 \\
\hline 2 & 11.641 & 0.264 & 885258 & 15236449 & 49.773 \\
\hline
\end{tabular}

Figure S79. Chiral HPLC chromatogram for racemic 3aa.

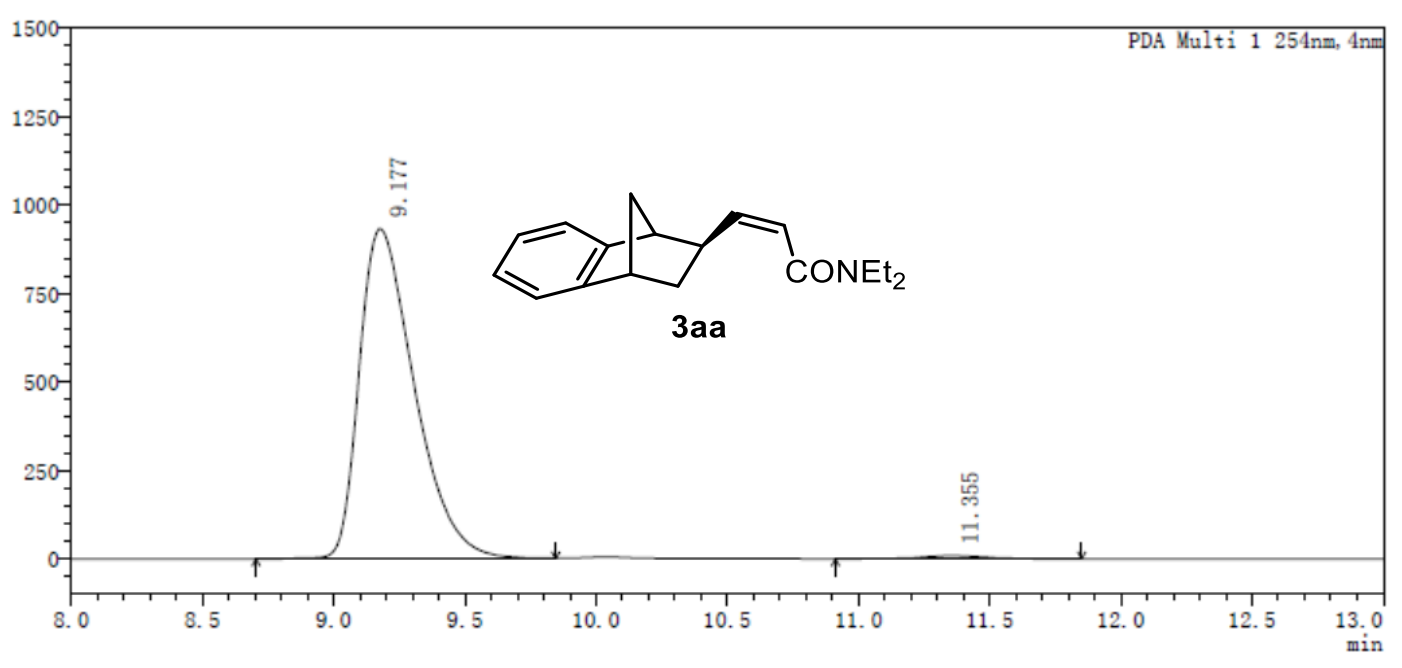

PDA Ch1 254nm

\begin{tabular}{|r|r|r|r|r|r|}
\hline 峰号 & 保留时间 & 峰宽 (高度 50\%) & \multicolumn{1}{c|}{ 高度 } & \multicolumn{1}{c|}{ 面积 } & \multicolumn{1}{c|}{ 面积\% } \\
\hline 1 & 9.177 & 0.223 & 933267 & 13645131 & 98.869 \\
\hline 2 & 11.355 & 0.255 & 8879 & 156029 & 1.131 \\
\hline
\end{tabular}

Figure S80. Chiral HPLC chromatogram for enantioenriched 3aa. 


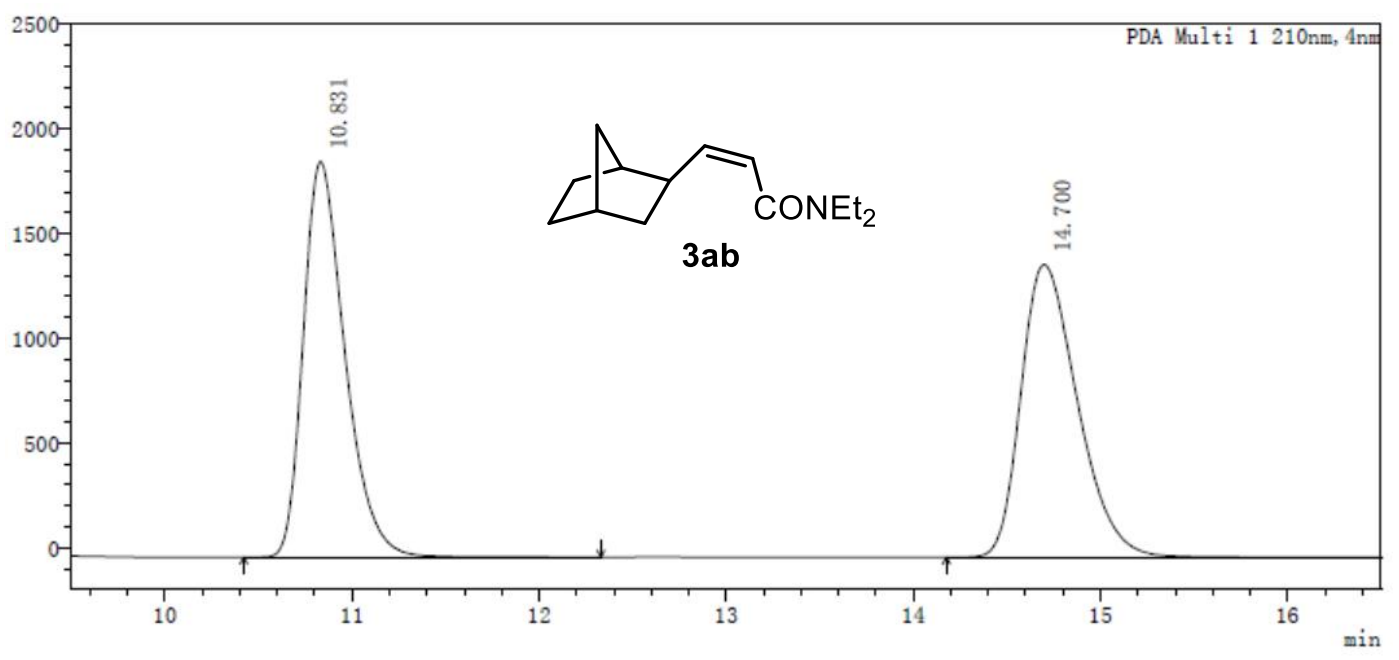

PDA Ch1 $210 \mathrm{~nm}$
\begin{tabular}{|r|r|r|l|l|l|}
\hline 峰号 & 保留时间 & 峰宽 (高度 $50 \%)$ & 高度 & 面积 & 面积\% \\
\hline 1 & 10.831 & 0.234 & 1886687 & 29229020 & 49.909 \\
\hline 2 & 14.700 & 0.323 & 1398179 & 29335252 & 50.091 \\
\hline
\end{tabular}

Figure S81. Chiral HPLC chromatogram for racemic 3ab.

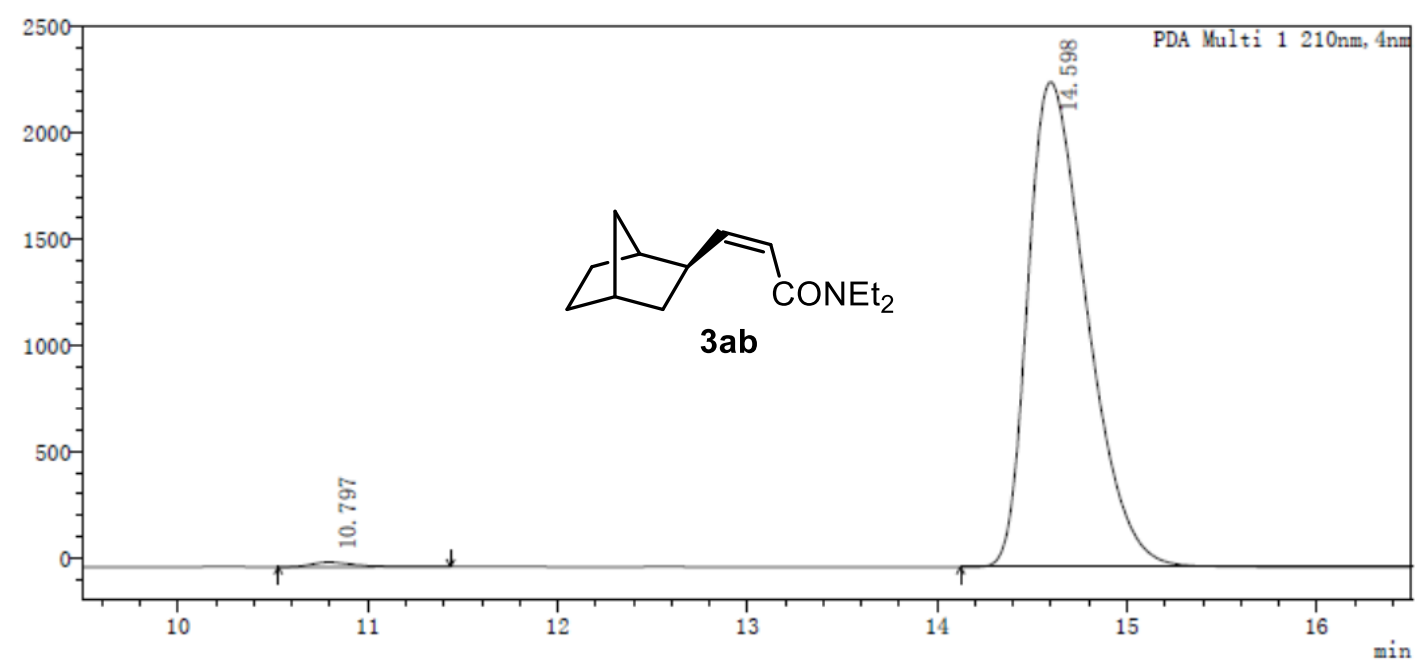

PDA Ch1 210nm

\begin{tabular}{|r|r|r|r|r|r|}
\hline 峰号 & 保留时间 & 峰宽 (高度 50\%) & \multicolumn{1}{|c|}{ 高度 } & \multicolumn{1}{|c|}{ 面积 } & \multicolumn{1}{c|}{ 面积\% } \\
\hline 1 & 10.797 & 0.230 & 21640 & 328383 & 0.662 \\
\hline 2 & 14.598 & 0.334 & 2283178 & 49306658 & 99.338 \\
\hline
\end{tabular}

Figure S82. Chiral HPLC chromatogram for enantioenriched 3ab. 


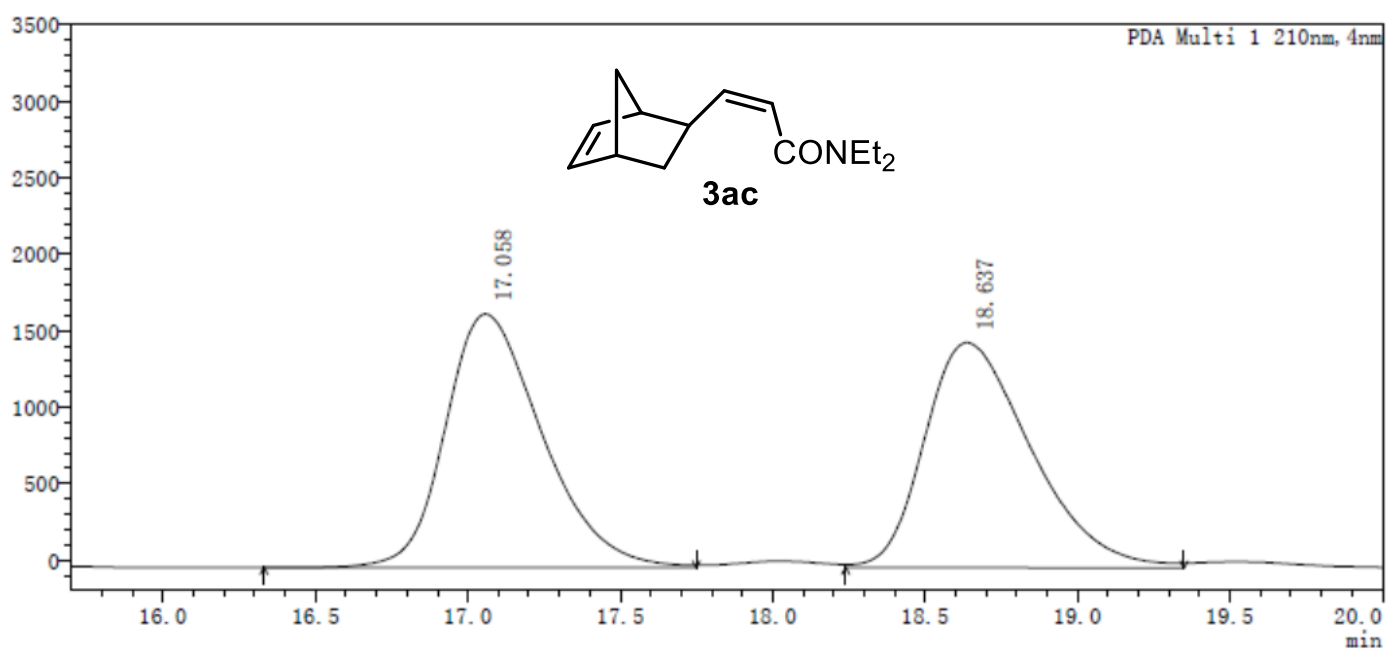

PDA Ch1 210nm

\begin{tabular}{|r|r|r|r|r|r|}
\hline 峰号 & 保留时间 & 峰宽 (高度 50\%) & 高度 & 面积 & 面积\% \\
\hline 1 & 17.058 & 0.336 & 1658039 & 36679881 & 50.590 \\
\hline 2 & 18.637 & 0.374 & 1471520 & 35825043 & 49.410 \\
\hline
\end{tabular}

Figure S83. Chiral HPLC chromatogram for racemic 3ac.

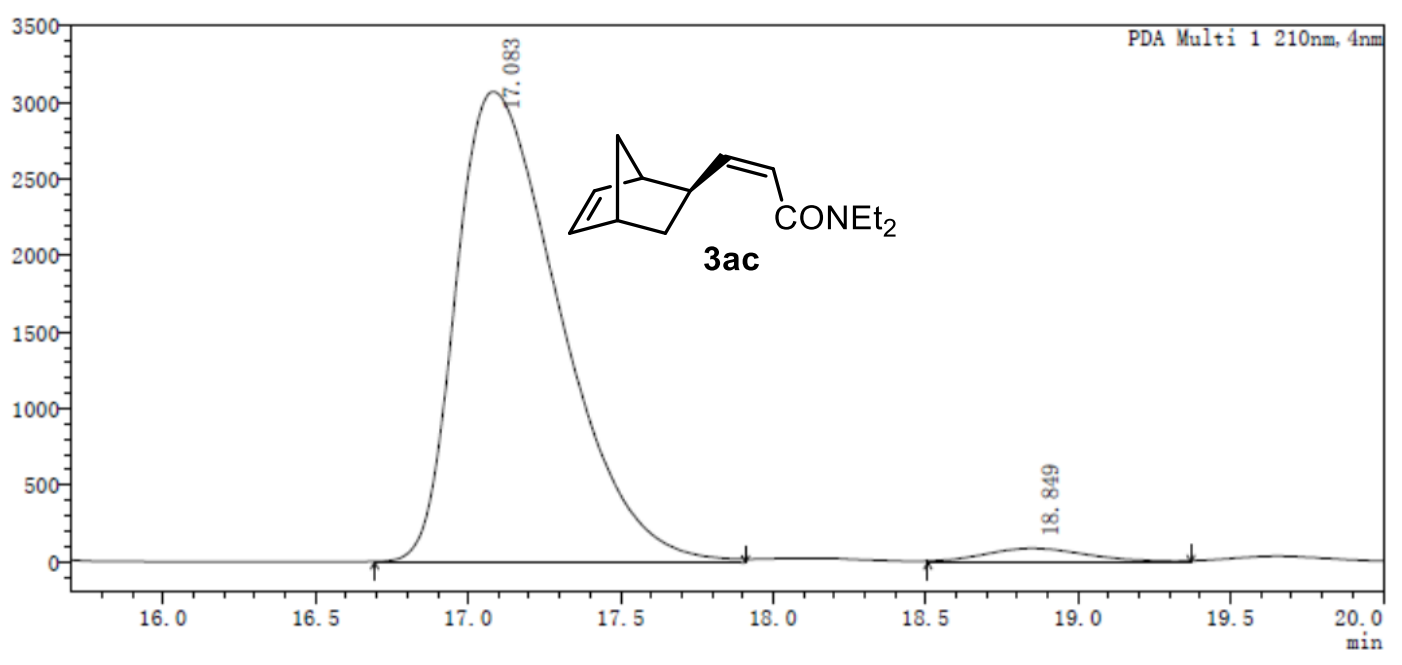

PDA Ch1 210nm

\begin{tabular}{|r|r|r|r|r|r|}
\hline r峰号 & 保留时间 & 峰宽 (高度 50\%) & 高度 & \multicolumn{1}{c|}{ 面积 } & \multicolumn{1}{c|}{ 面积\% } \\
\hline 1 & 17.083 & 0.368 & 3073059 & 72999628 & 97.044 \\
\hline 2 & 18.849 & 0.370 & 91091 & 2223281 & 2.956 \\
\hline
\end{tabular}

Figure S84. Chiral HPLC chromatogram for enantioenriched 3ac. 


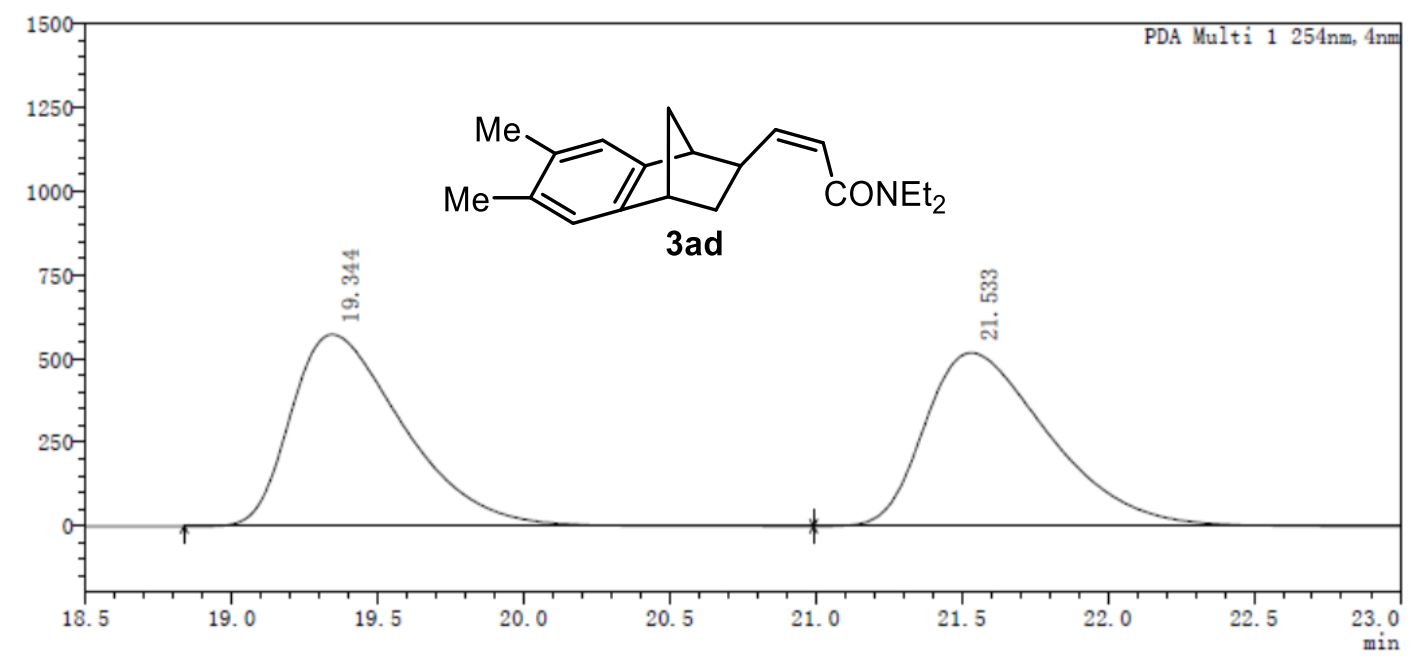

PDA Ch1 $254 \mathrm{~nm}$
\begin{tabular}{|r|r|r|r|c|c|}
\hline 峰号 & 保留时间 & 峰宽 (高度 $50 \%$ ) & 高度 & 面积 & 面积\% \\
\hline 1 & 19.344 & 0.412 & 573433 & 15427315 & 50.028 \\
\hline 2 & 21.533 & 0.457 & 518513 & 15409917 & 49.972 \\
\hline
\end{tabular}

Figure S85. Chiral HPLC chromatogram for racemic 3ad.

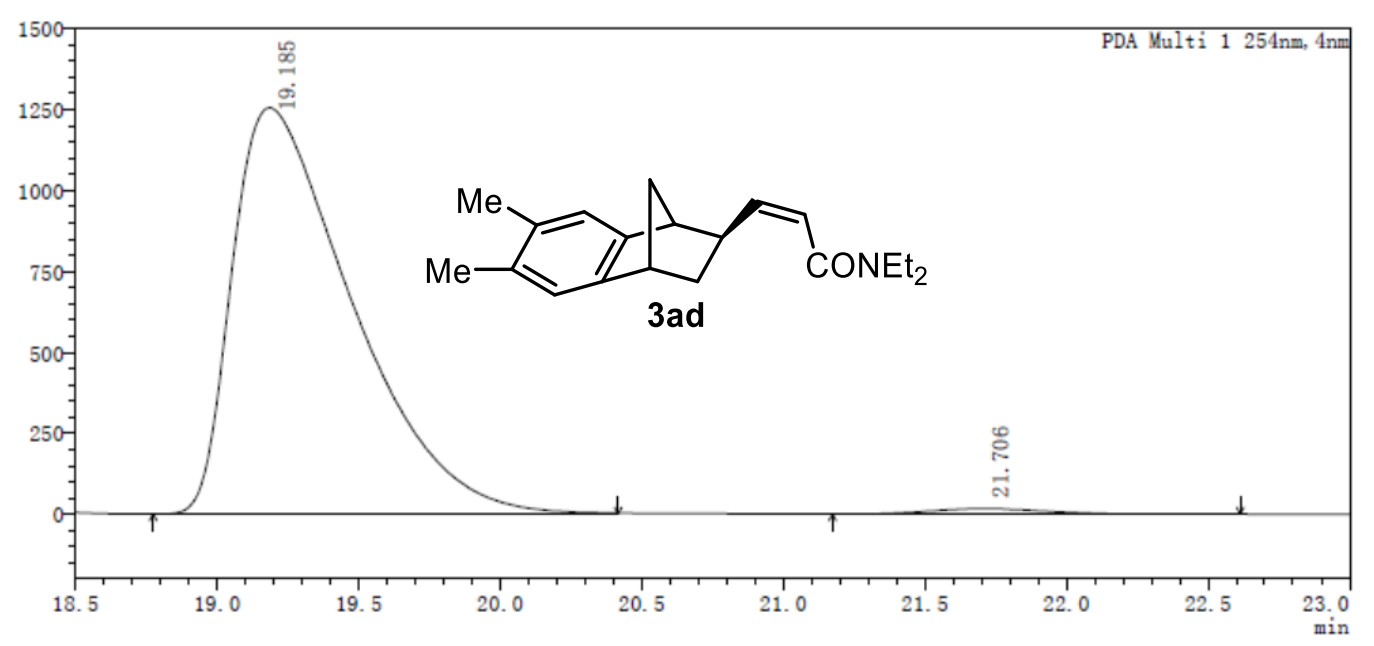

\begin{tabular}{|c|c|c|c|c|c|}
\hline \\
\hline 峰号 & 保留时间 & 峰宽 (高度 $50 \%$ ) & 高度 & 面积 & 面积\% \\
\hline 1 & 19.185 & 0.452 & 1255832 & 37020474 & 98.776 \\
\hline 2 & 21.706 & 0.427 & 16678 & 458705 & 1. 224 \\
\hline
\end{tabular}

Figure S86. Chiral HPLC chromatogram for enantioenriched 3ad. 


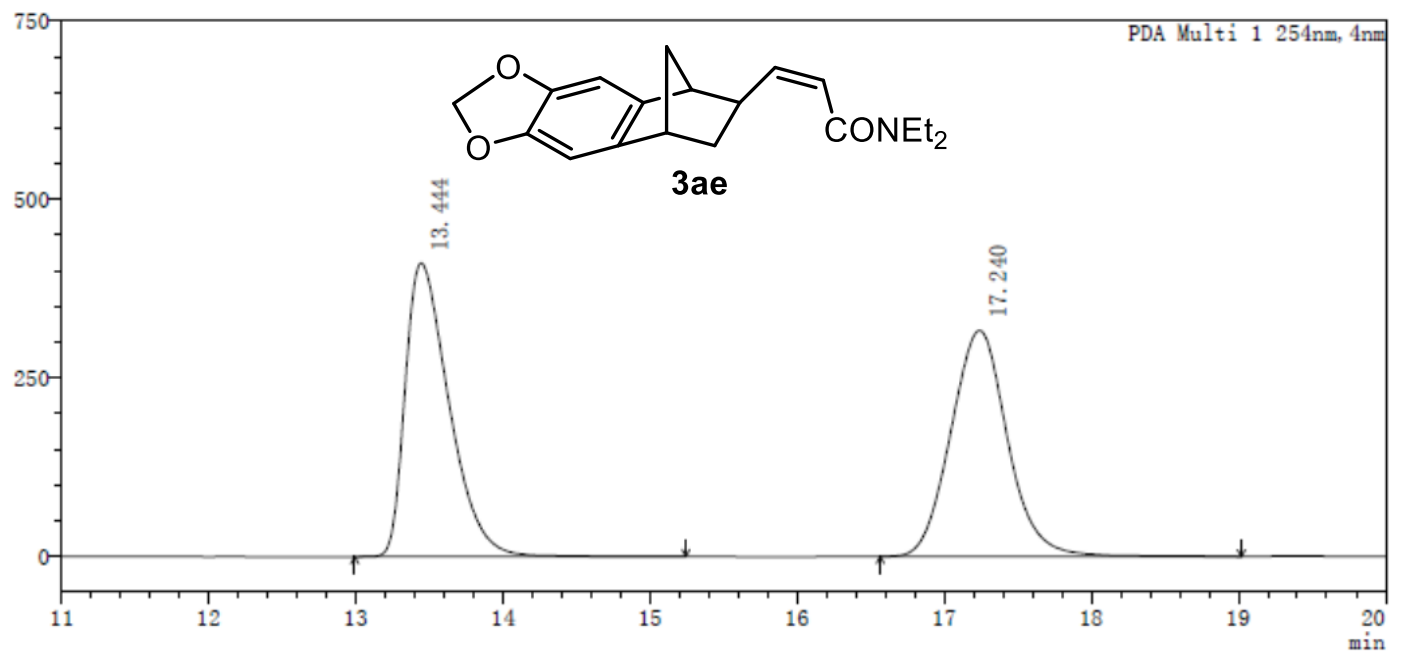

PDA Ch1 $254 \mathrm{~nm}$
\begin{tabular}{|r|r|r|r|r|r|}
\hline 峰号 & 保留时间 & 峰宽 (高度 50\%) & 高度 & \multicolumn{1}{l|}{ 面积 } & 面积\% \\
\hline 1 & 13.444 & 0.317 & 411013 & 8508820 & 49.970 \\
\hline 2 & 17.240 & 0.408 & 316607 & 8518922 & 50.030 \\
\hline
\end{tabular}

Figure S87. Chiral HPLC chromatogram for racemic 3ae.

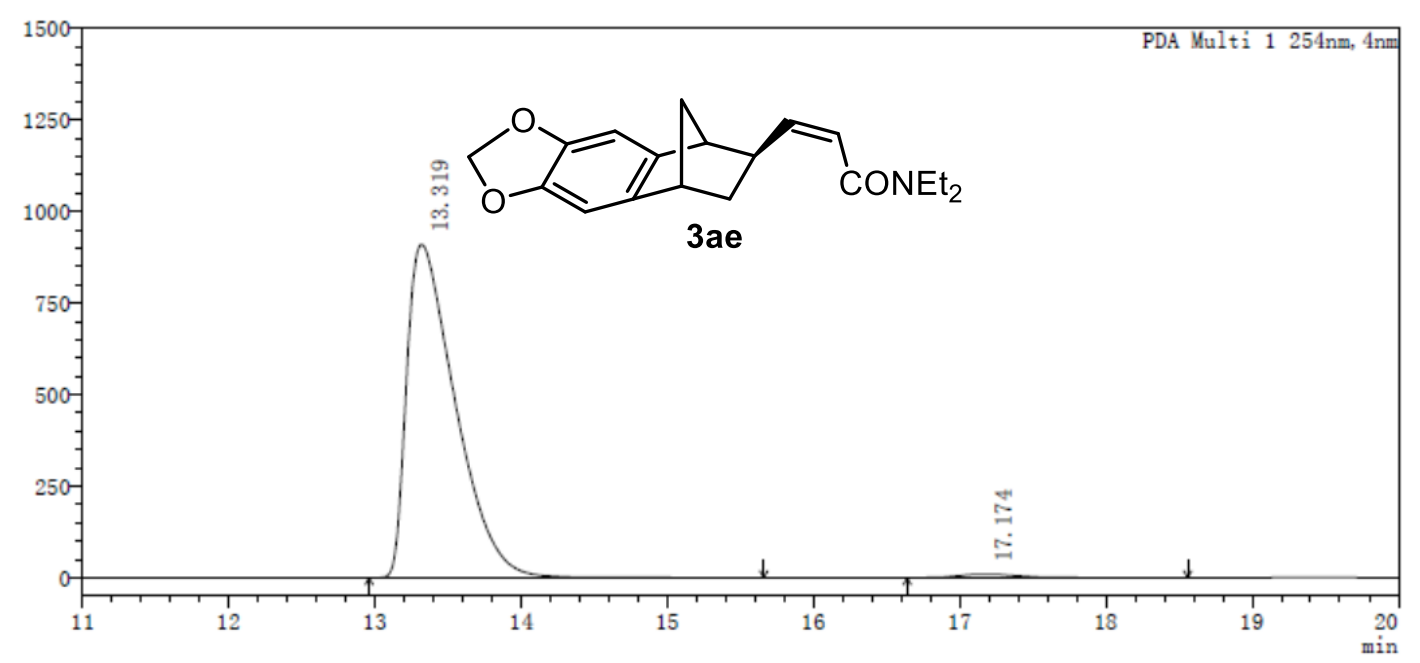

PDA Ch1 254nm

\begin{tabular}{|r|r|r|r|r|r|}
\hline 峰号 & 保留时间 & 峰宽 (高度 50\%) & 高度 & \multicolumn{1}{c|}{ 面积 } & \multicolumn{1}{c|}{ 面积\% } \\
\hline 1 & 13.319 & 0.357 & 910140 & 21085122 & 98.703 \\
\hline 2 & 17.174 & 0.423 & 9817 & 277153 & 1.297 \\
\hline
\end{tabular}

Figure S88. Chiral HPLC chromatogram for enantioenriched 3ae. 


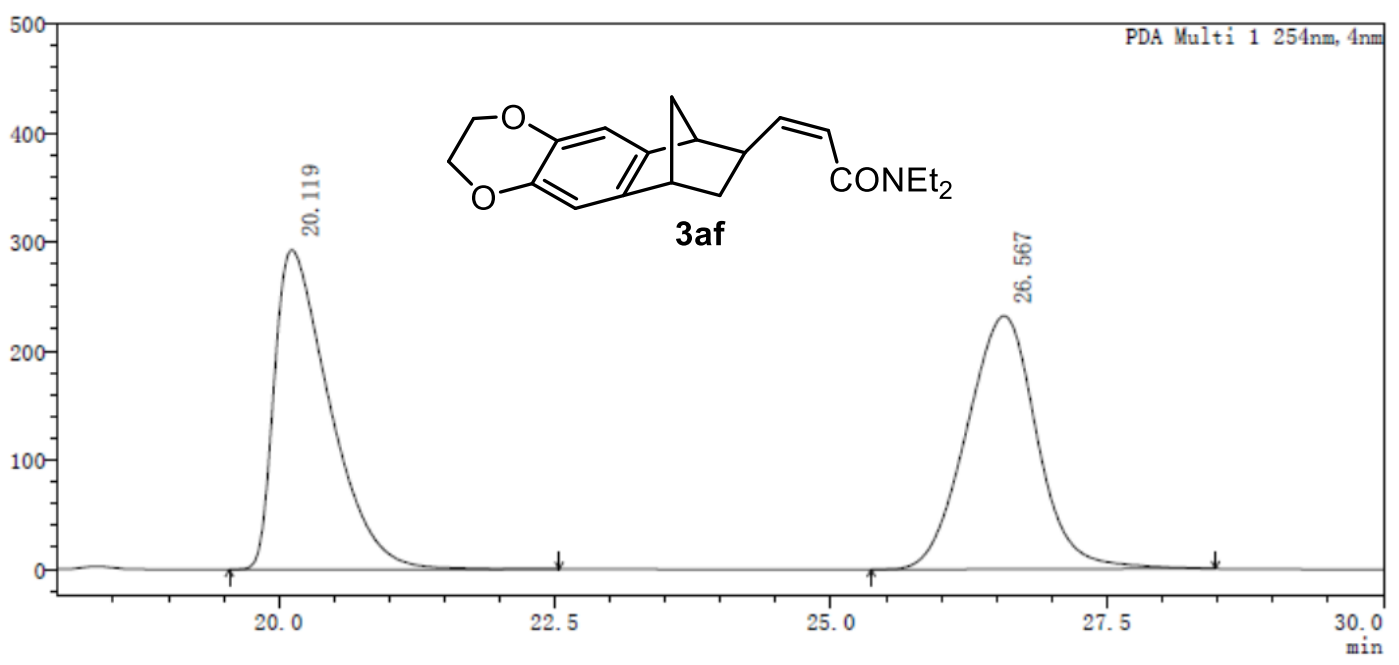

PDA Ch1 254nm

\begin{tabular}{|r|r|r|r|r|r|}
\hline 峰号 & 保留时间 & 峰宽 (高度 50\%) & 高度 & 面积 & \multicolumn{1}{c|}{ 面积\% } \\
\hline 1 & 20.119 & 0.532 & 293169 & 10227864 & 49.795 \\
\hline 2 & 26.567 & 0.677 & 232391 & 10312028 & 50.205 \\
\hline
\end{tabular}

Figure S89. Chiral HPLC chromatogram for racemic 3af.

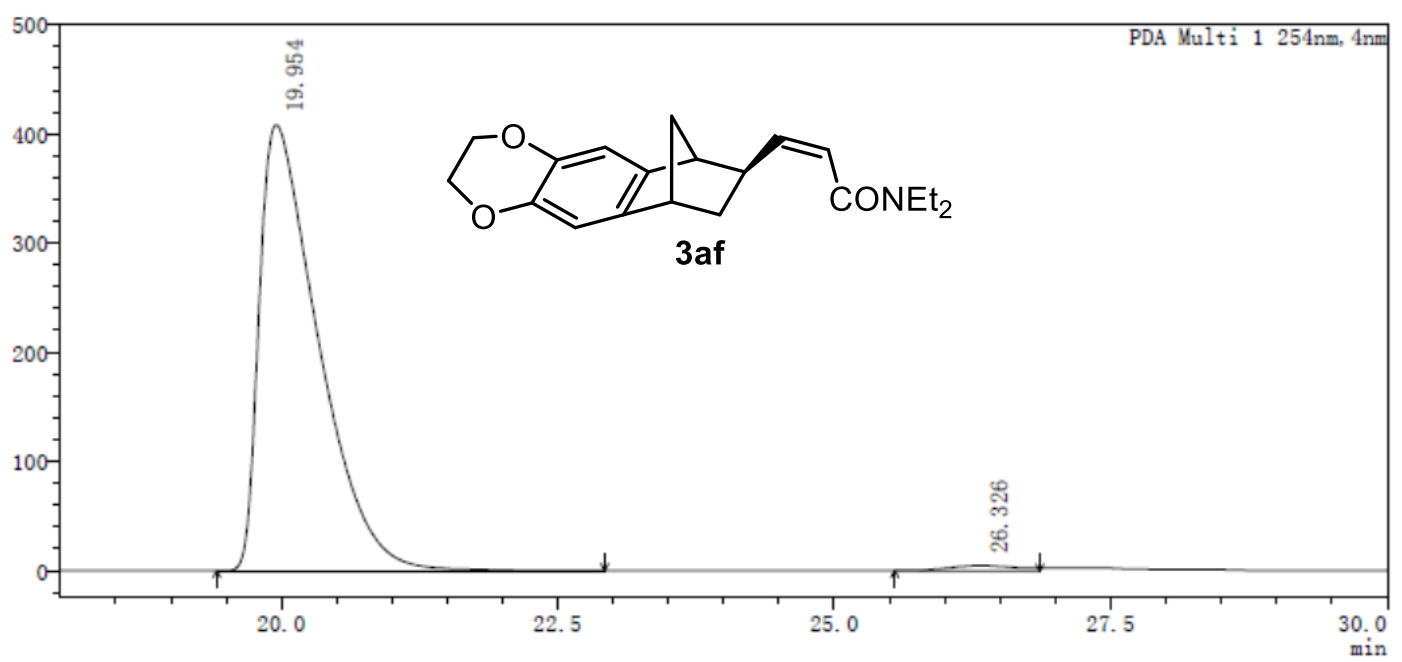

PDA Ch1 254nm

\begin{tabular}{|r|r|r|r|r|r|}
\hline 峰号 & 保留时间 & 峰宽 (高度 50\%) & 高度 & \multicolumn{1}{c|}{ 面积 } & \multicolumn{1}{c|}{ 面积\% } \\
\hline 1 & 19.954 & 0.566 & 408430 & 15159890 & 98.662 \\
\hline 2 & 26.326 & 0.775 & 4787 & 205524 & 1.338 \\
\hline
\end{tabular}

Figure S90. Chiral HPLC chromatogram for enantioenriched 3af. 


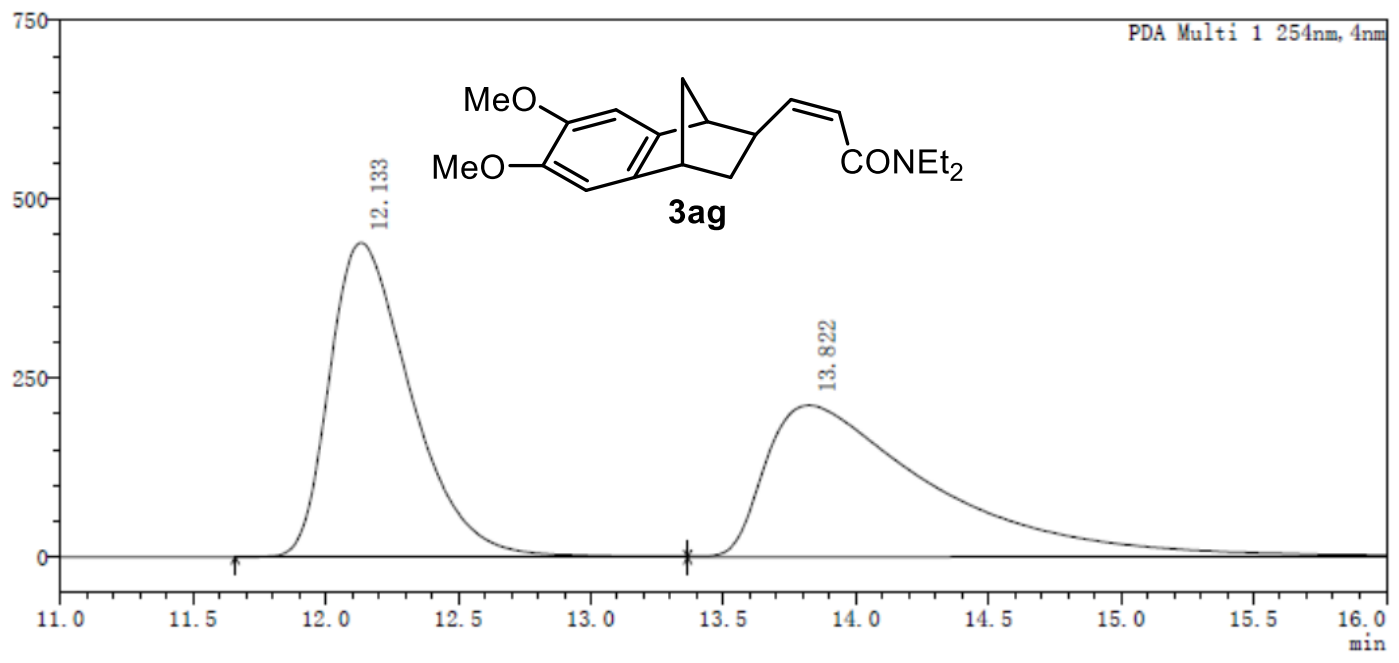

\begin{tabular}{|c|c|c|c|c|c|}
\hline 峰号 & 保留时间 & 峰宽 (高度 50\%) & 高度 & 面积 & 面积\% \\
\hline 1 & 12.133 & 0.327 & 438676 & 9410348 & 50.000 \\
\hline 2 & 13.822 & 0.628 & 211668 & 9410162 & 50.000 \\
\hline
\end{tabular}

Figure S91. Chiral HPLC chromatogram for racemic 3ag.

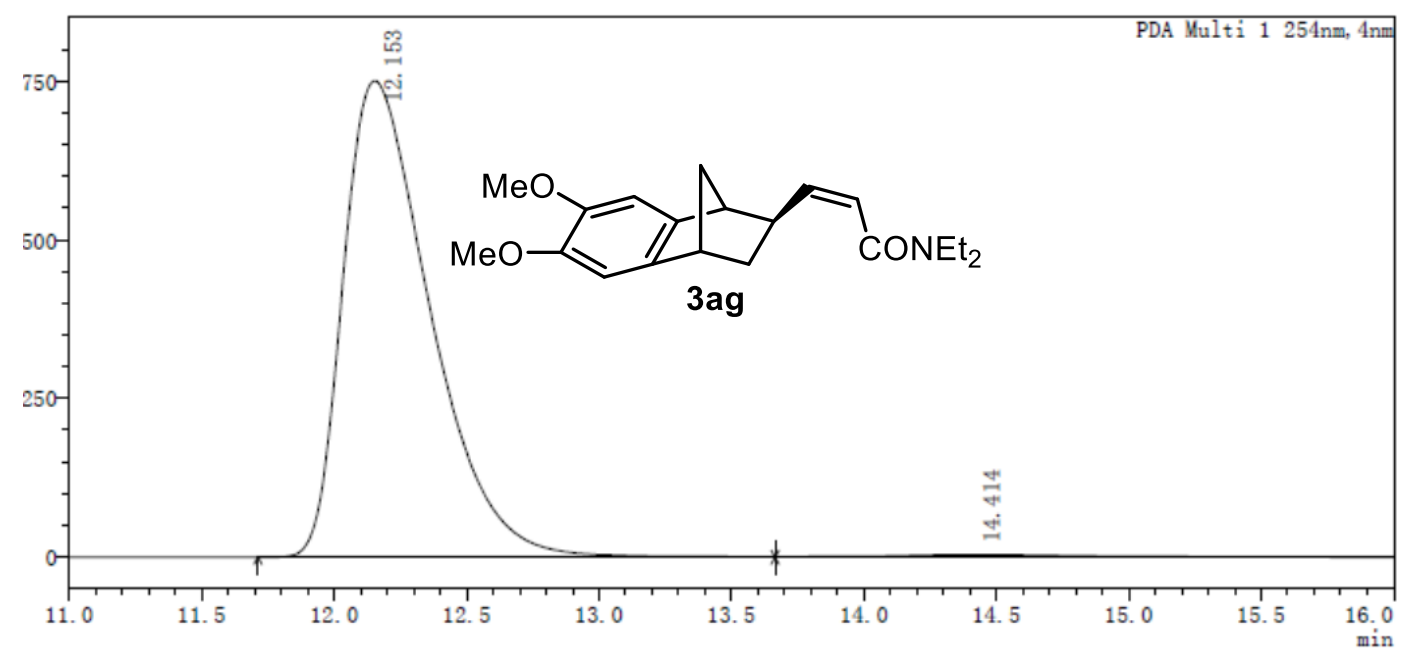

PDA Ch1 254nm

\begin{tabular}{|r|r|r|r|r|r|}
\hline 峰号 & 保留时间 & 峰宽 (高度 50\%) & \multicolumn{1}{c|}{ 高度 } & \multicolumn{1}{c|}{ 面积 } & \multicolumn{1}{c|}{ 面积\% } \\
\hline 1 & 12.153 & 0.349 & 750073 & 17153617 & 98.735 \\
\hline 2 & 14.414 & 0.877 & 3569 & 219757 & 1.265 \\
\hline
\end{tabular}

Figure S92. Chiral HPLC chromatogram for enantioenriched 3ag. 


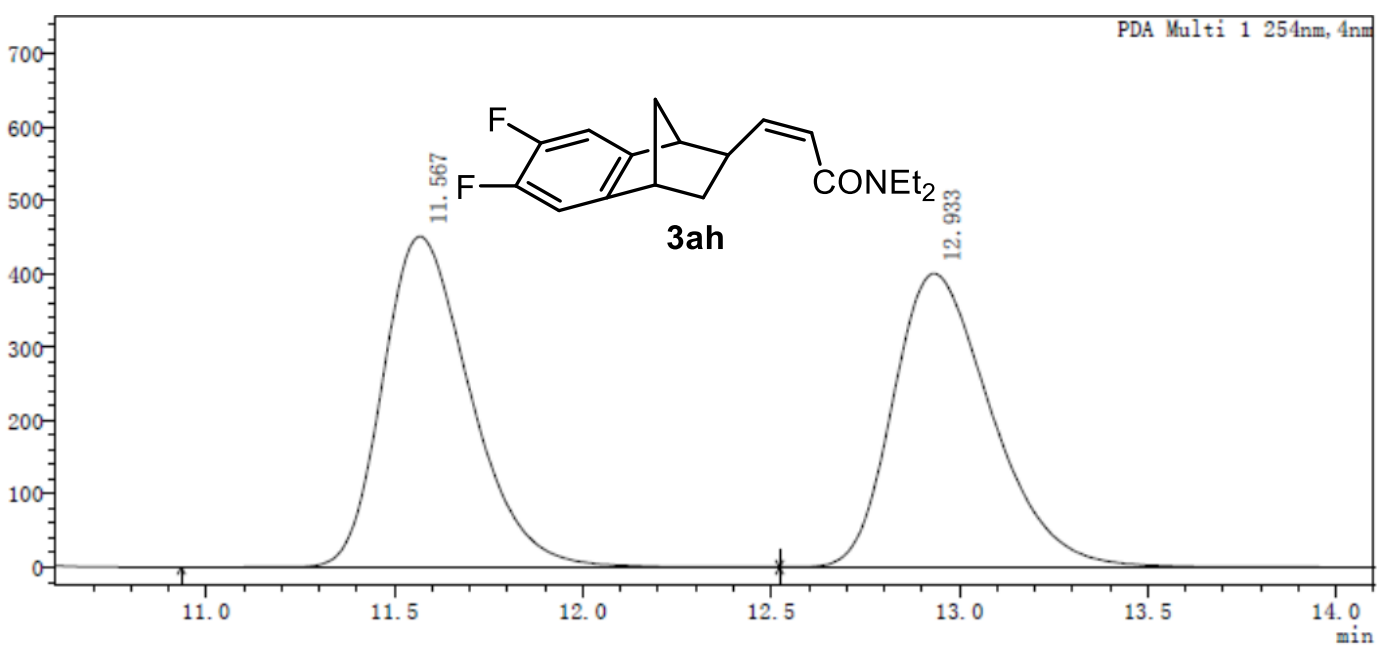

PDA Ch1 254nm

\begin{tabular}{|r|r|r|r|r|r|}
\hline 峰号 & 保留时间 & 峰宽 (高度 50\%) & 高度 & 面积 & \multicolumn{1}{c|}{ 面积\% } \\
\hline 1 & 11.567 & 0.248 & 450656 & 7321947 & 50.023 \\
\hline 2 & 12.933 & 0.280 & 400050 & 7315170 & 49.977 \\
\hline
\end{tabular}

Figure S93. Chiral HPLC chromatogram for racemic 3ah.

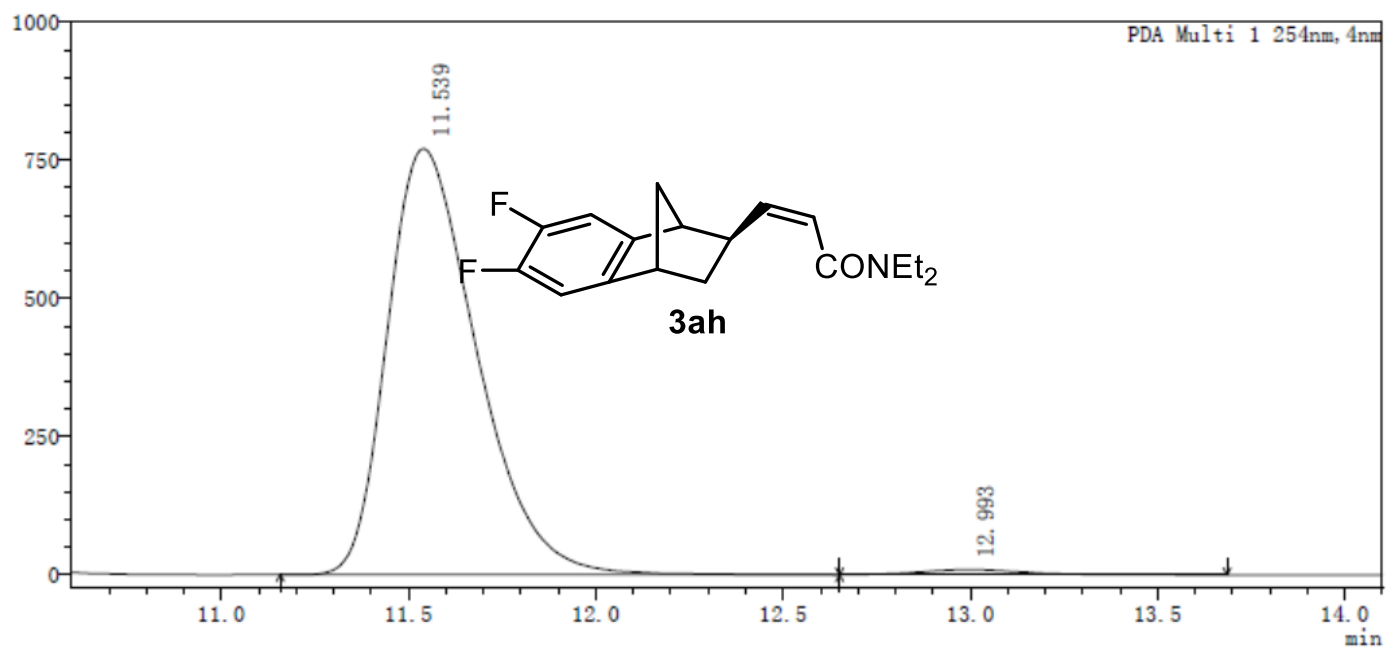

PDA Ch1 254nm

\begin{tabular}{|r|r|r|r|r|r|}
\hline 峰号 & 保留时间 & 峰宽 (高度 50\%) & 高度 & \multicolumn{1}{c|}{ 面积 } & \multicolumn{1}{c|}{ 面积\% } \\
\hline 1 & 11.539 & 0.255 & 771082 & 12811232 & 98.746 \\
\hline 2 & 12.993 & 0.275 & 9025 & 162694 & 1.254 \\
\hline
\end{tabular}

Figure S94. Chiral HPLC chromatogram for enantioenriched 3ah. 


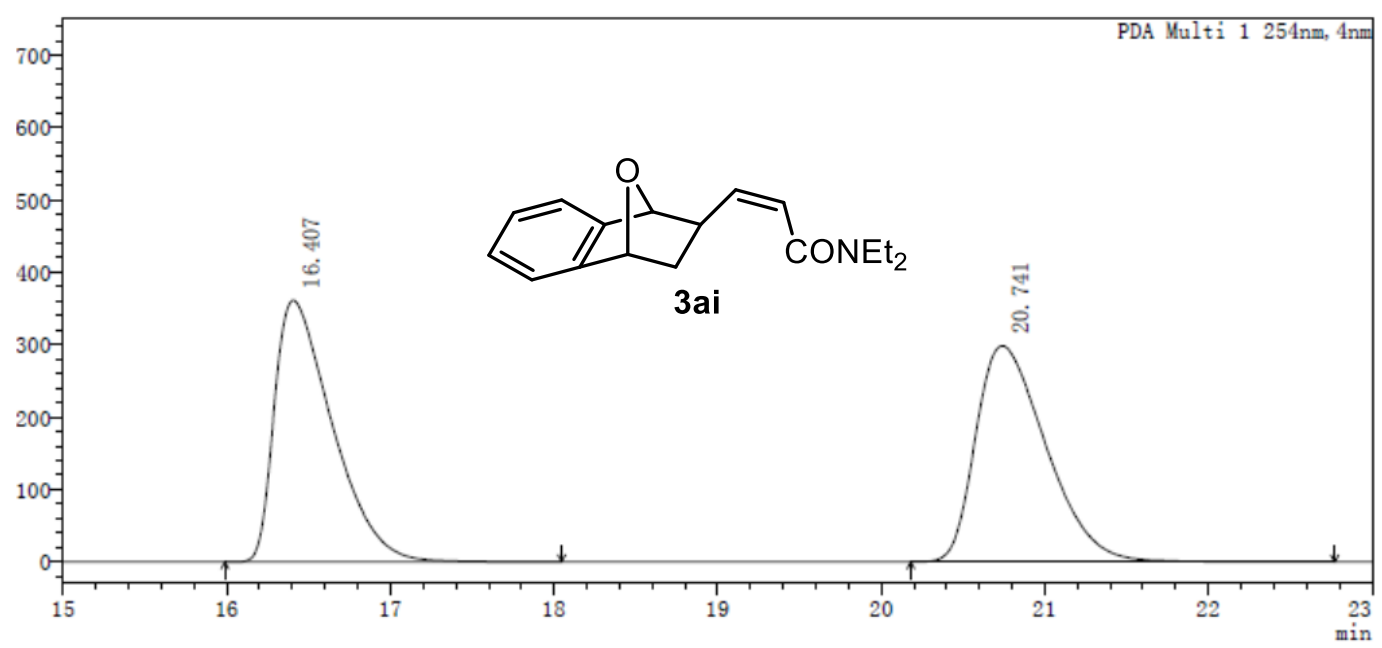

PDA Ch1 254nm

\begin{tabular}{|r|r|r|r|r|r|} 
PDA Ch1 $254 \mathrm{~nm}$ \\
\hline 峰号 & 保留时间 & 峰宽 (高度 50\%) & 高度 & 面积 & 面积\% \\
\hline 1 & 16.407 & 0.379 & 361453 & 8889818 & 49.896 \\
\hline 2 & 20.741 & 0.466 & 298442 & 8926864 & 50.104 \\
\hline
\end{tabular}

Figure S95. Chiral HPLC chromatogram for racemic 3ai.

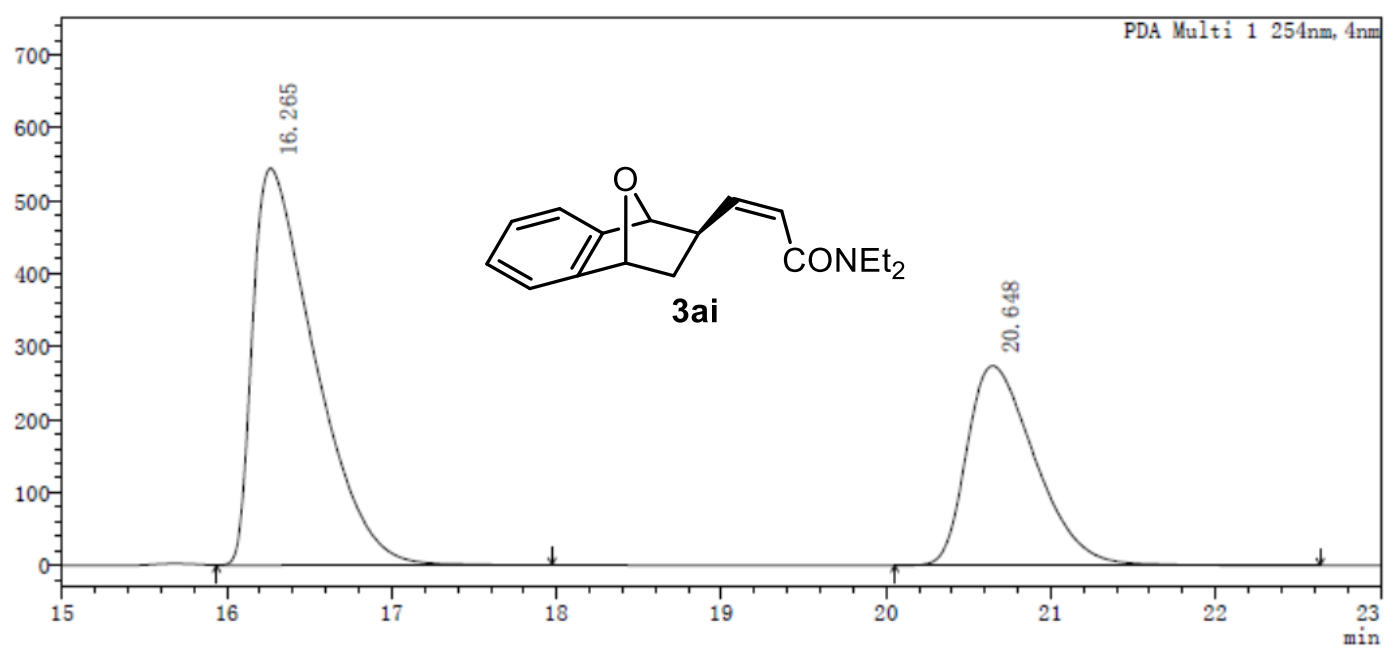

PDA Ch1 254nm

\begin{tabular}{|r|r|r|r|r|r|}
\hline 峰号 & 保留时间 & 峰宽 (高度 50\%) & \multicolumn{1}{|c|}{ 高度 } & \multicolumn{1}{c|}{ 面积 } & \multicolumn{1}{c|}{ 面积\% } \\
\hline 1 & 16.265 & 0.404 & 543875 & 14231710 & 64.362 \\
\hline 2 & 20.648 & 0.447 & 273446 & 7880340 & 35.638 \\
\hline
\end{tabular}

Figure S96. Chiral HPLC chromatogram for enantioenriched 3ai. 


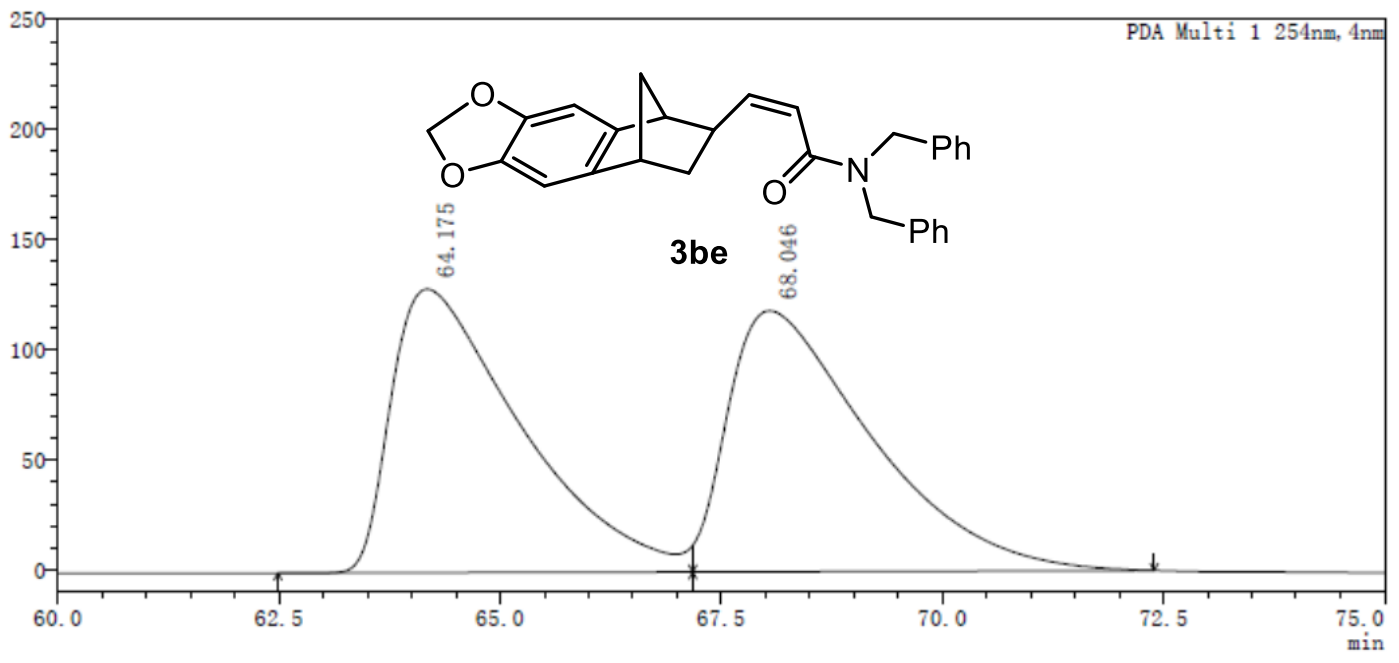

PDA Ch1 $254 \mathrm{~nm}$
\begin{tabular}{|r|r|r|r|r|r|}
\hline 峰号 & 保留时间 & 峰宽 (高度 50\%) & 高度 & 面积 & 面积 $\%$ \\
\hline 1 & 64.175 & 1.559 & 128637 & 13182322 & 49.673 \\
\hline 2 & 68.046 & 1.726 & 118349 & 13356080 & 50.327 \\
\hline
\end{tabular}

Figure S97. Chiral HPLC chromatogram for racemic $3 b e$.

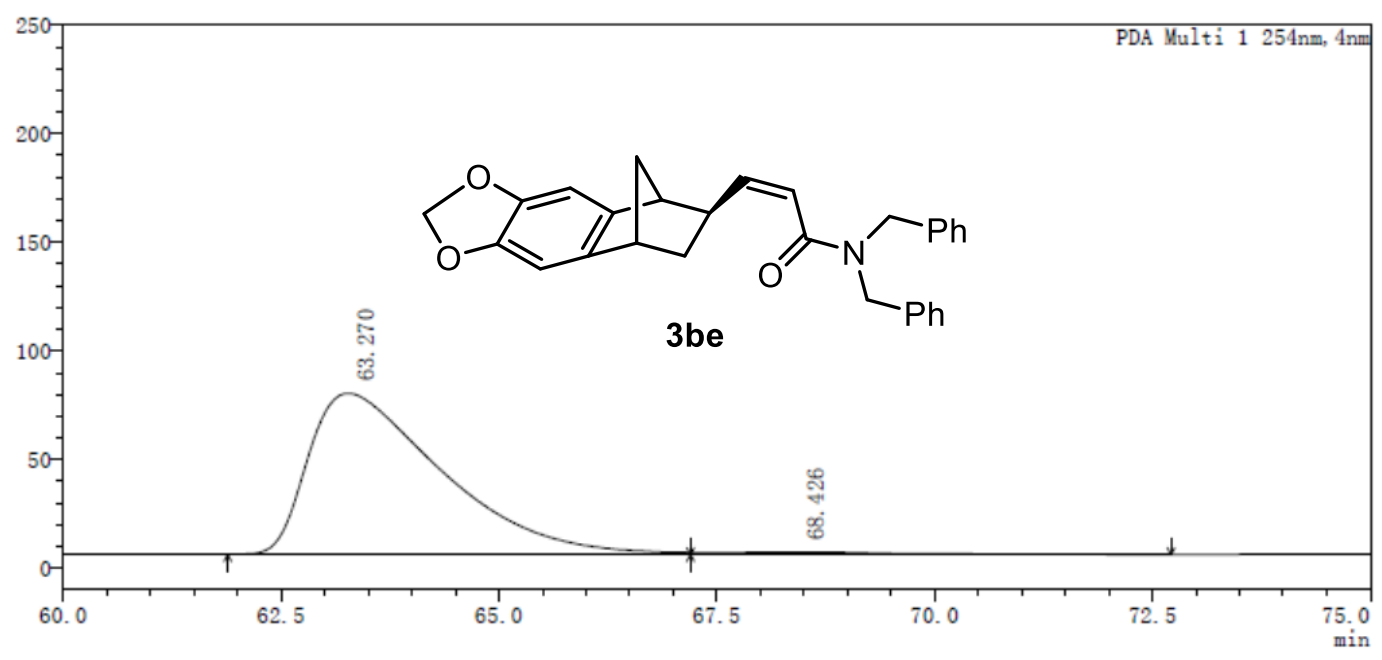

PDA Ch1 254nm

\begin{tabular}{|r|r|r|r|r|r|}
\hline 峰号 & 保留时间 & 峰宽 (高度 50\%) & 高度 & \multicolumn{1}{c|}{ 面积 } & \multicolumn{1}{c|}{ 面积\% } \\
\hline 1 & 63.270 & 1.629 & 74161 & 7913449 & 98.098 \\
\hline 2 & 68.426 & -- & 1065 & 153458 & 1.902 \\
\hline
\end{tabular}

Figure S98. Chiral HPLC chromatogram for enantioenriched 3be. 


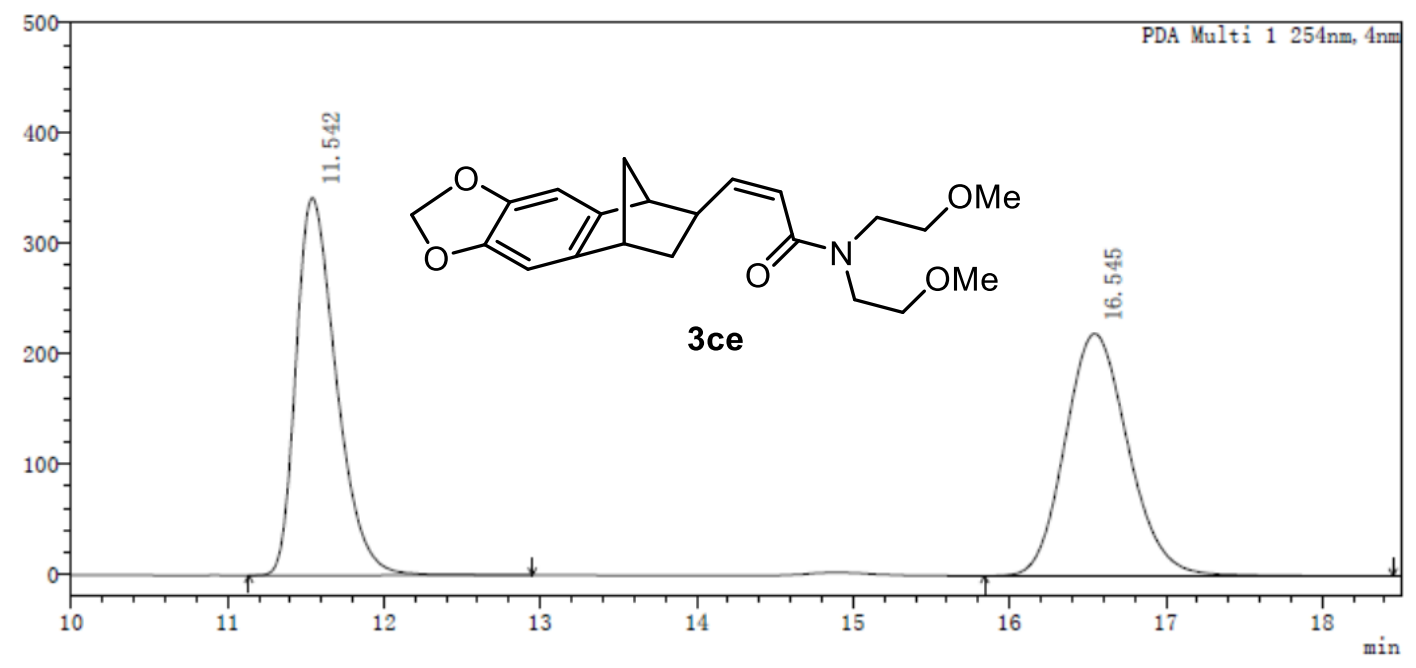

PDA Ch1 254nm

\begin{tabular}{|r|r|r|r|r|r|}
\hline 峰号 & 保留时间 & 峰宽 (高度 50\%) & 高度 & 面积 & \multicolumn{1}{c|}{ 面积\% } \\
\hline 1 & 11.542 & 0.273 & 342196 & 6131462 & 49.942 \\
\hline 2 & 16.545 & 0.432 & 219459 & 6145806 & 50.058 \\
\hline
\end{tabular}

Figure S99. Chiral HPLC chromatogram for racemic 3ce.

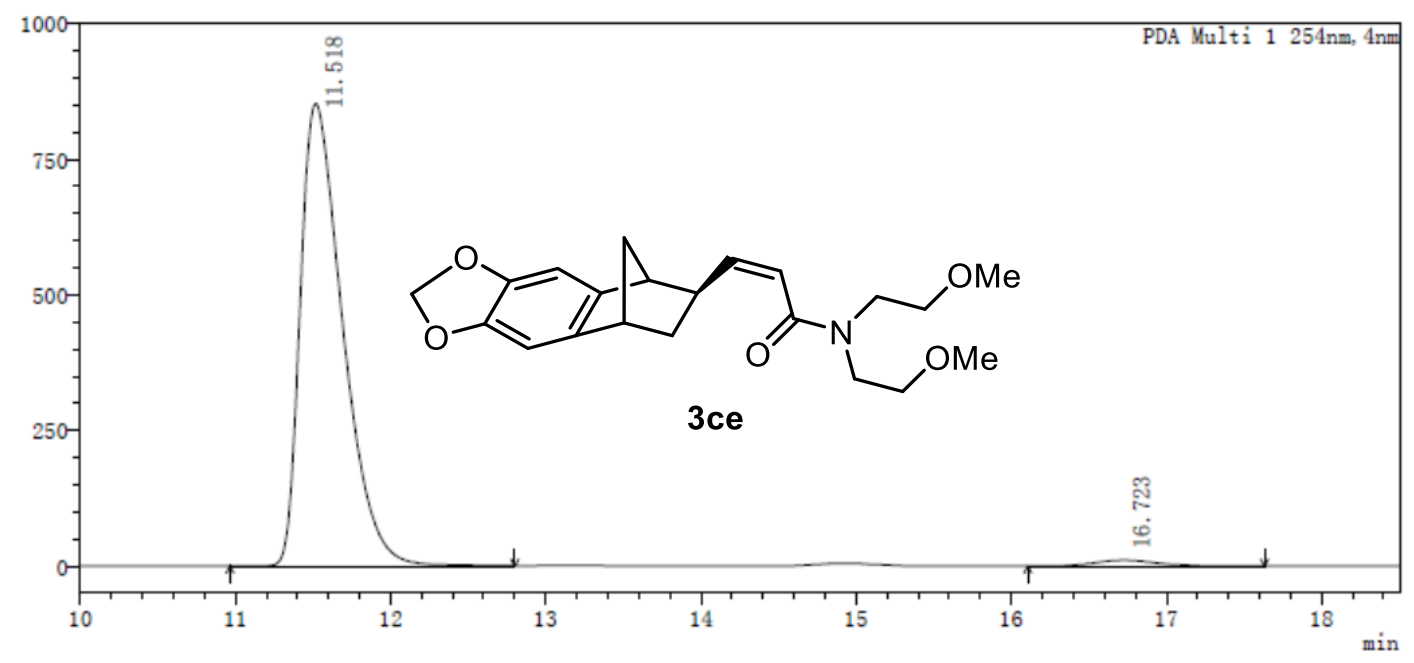

PDA Ch1 254nm

\begin{tabular}{|r|r|r|r|r|r|}
\hline 峰号 & 保留时间 & 峰宽 (高度 50\%) & \multicolumn{1}{c|}{ 高度 } & \multicolumn{1}{c|}{ 面积 } & \multicolumn{1}{c|}{ 面积\% } \\
\hline 1 & 11.518 & 0.291 & 852823 & 16201337 & 98.122 \\
\hline 2 & 16.723 & 0.426 & 11002 & 310102 & 1.878 \\
\hline
\end{tabular}

Figure S100. Chiral HPLC chromatogram for enantioenriched 3ce. 


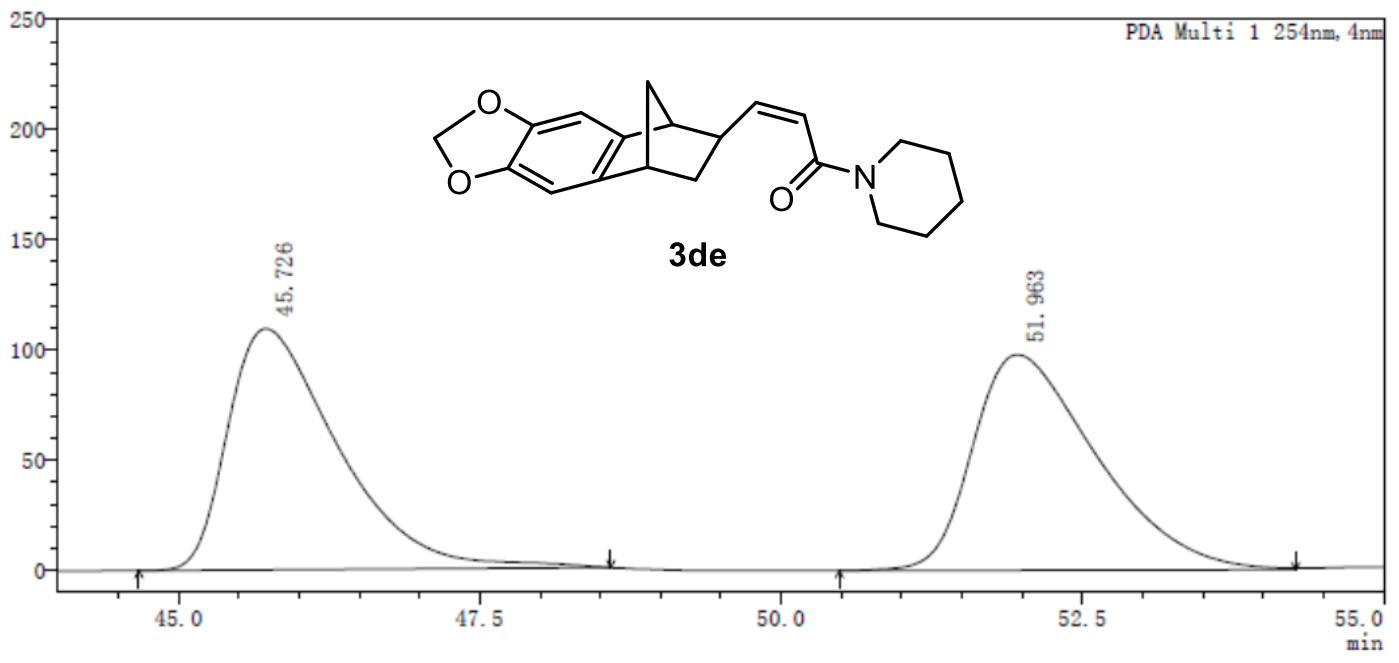

PDA Ch1 254nm

\begin{tabular}{|r|r|r|r|r|r|}
\hline 峰号 & 保留时间 & 峰宽 (高度 50\%) & 高度 & 面积 & \multicolumn{1}{c|}{ 面积\% } \\
\hline 1 & 45.726 & 0.962 & 109350 & 6986376 & 49.927 \\
\hline 2 & 51.963 & 1.102 & 97729 & 7006700 & 50.073 \\
\hline
\end{tabular}

Figure S101. Chiral HPLC chromatogram for racemic 3de.

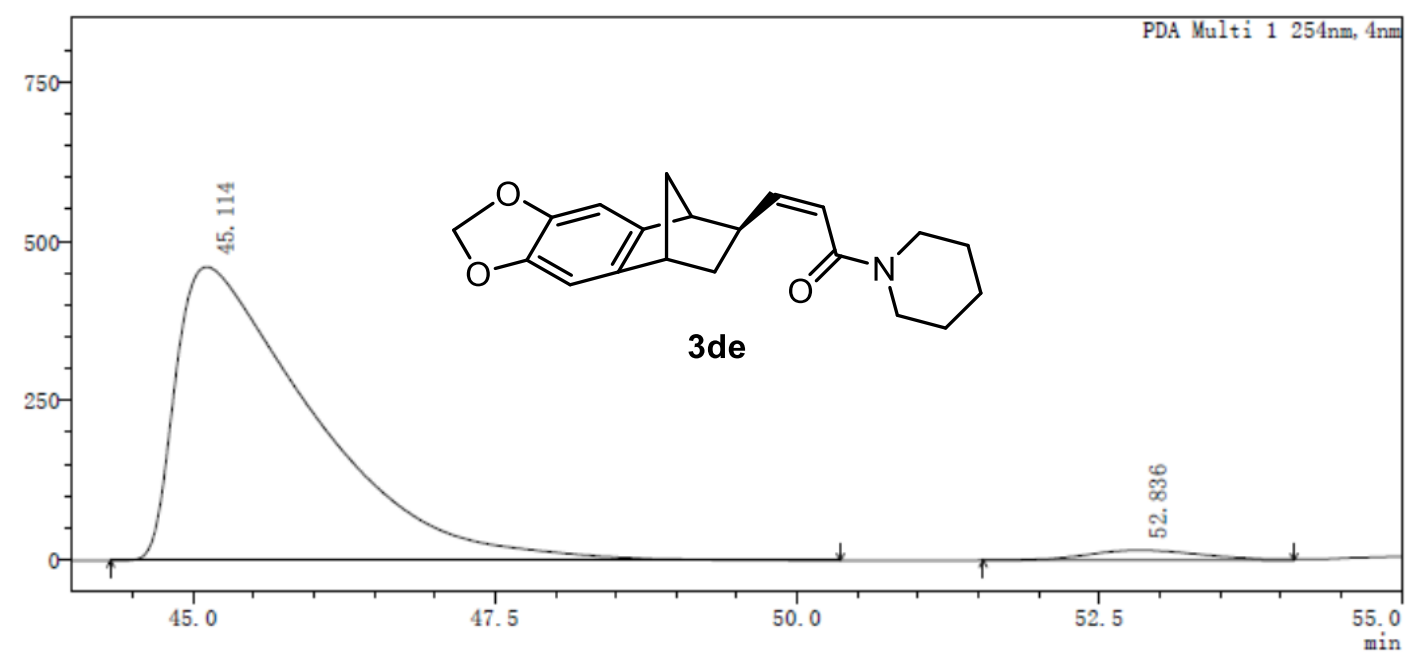

PDA Ch1 254nm

\begin{tabular}{|r|r|r|r|r|r|}
\hline 峰号 & 保留时间 & 峰宽 (高度 50\%) & 高度 & \multicolumn{1}{c|}{ 面积 } & 面积\% \\
\hline 1 & 45.114 & 1.177 & 460000 & 36409474 & 97.206 \\
\hline 2 & 52.836 & 1.033 & 15807 & 1046577 & 2.794 \\
\hline
\end{tabular}

Figure S102. Chiral HPLC chromatogram for enantioenriched 3de. 


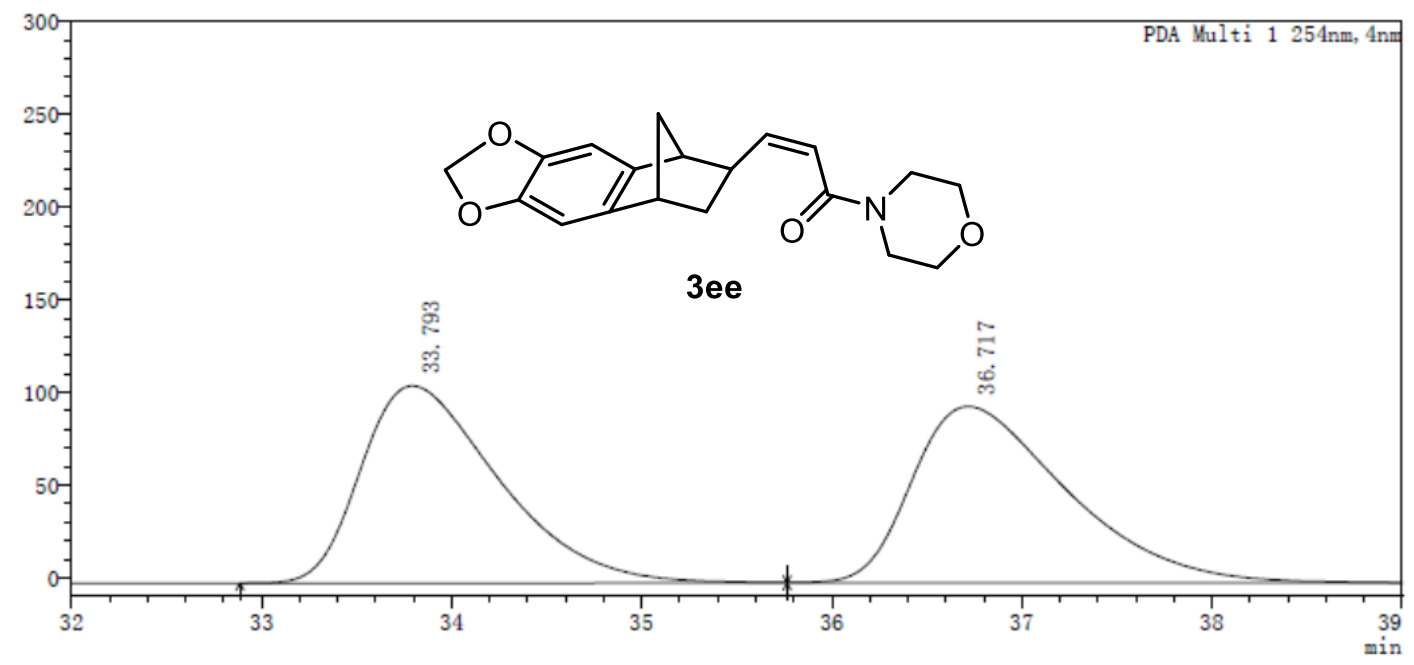

PDA Ch1 254nm

\begin{tabular}{|r|r|r|r|r|r|}
\hline 峰号 & 保留时间 & 峰宽 (高度 50\%) & 高度 & 面积 & \multicolumn{1}{c|}{ 面积\% } \\
\hline 1 & 33.793 & 0.776 & 106360 & 5372645 & 49.893 \\
\hline 2 & 36.717 & 0.870 & 95075 & 5395587 & 50.107 \\
\hline
\end{tabular}

Figure S103. Chiral HPLC chromatogram for racemic 3ee.

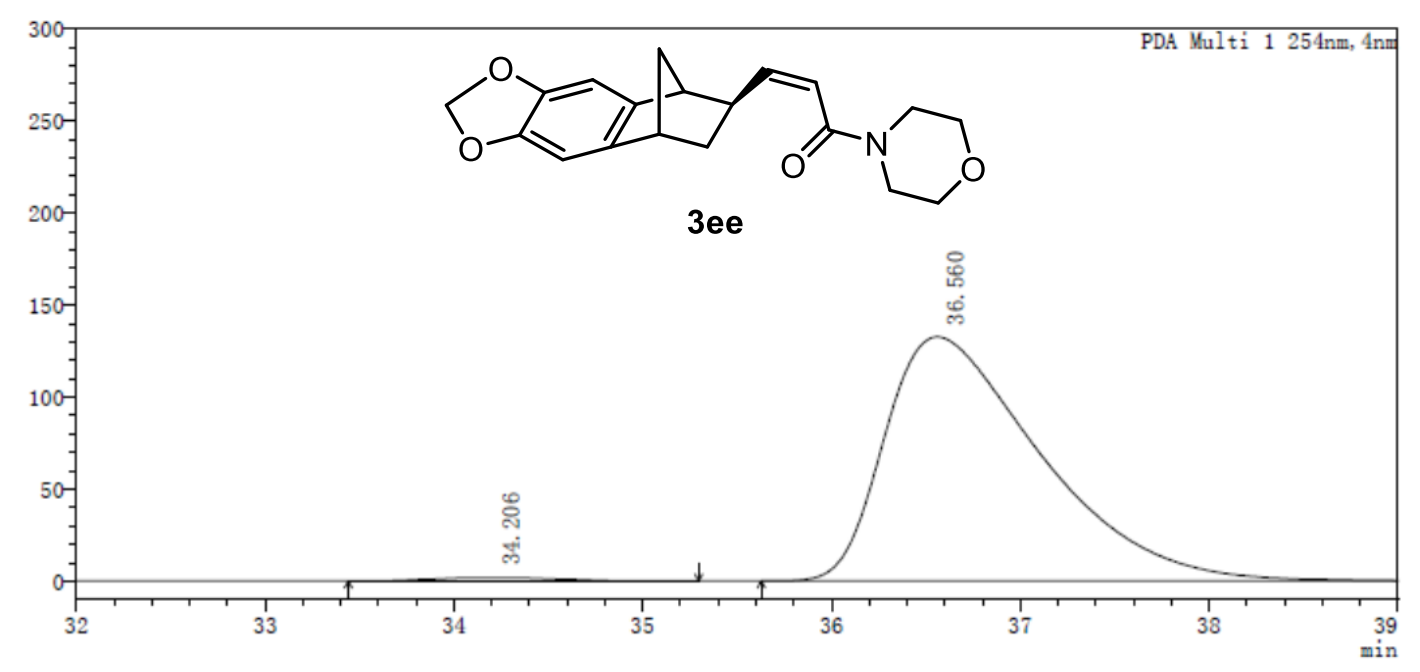

PDA Ch1 254nm

\begin{tabular}{|r|r|r|r|r|r|}
\hline 峰号 & 保留时间 & 峰宽 (高度 50\%) & \multicolumn{1}{c|}{ 高度 } & \multicolumn{1}{c|}{ 面积 } & \multicolumn{1}{c|}{ 面积\% } \\
\hline 1 & 34.206 & 0.720 & 1879 & 85782 & 1.115 \\
\hline 2 & 36.560 & 0.879 & 132486 & 7607144 & 98.885 \\
\hline
\end{tabular}

Figure S104. Chiral HPLC chromatogram for enantioenriched 3ee. 


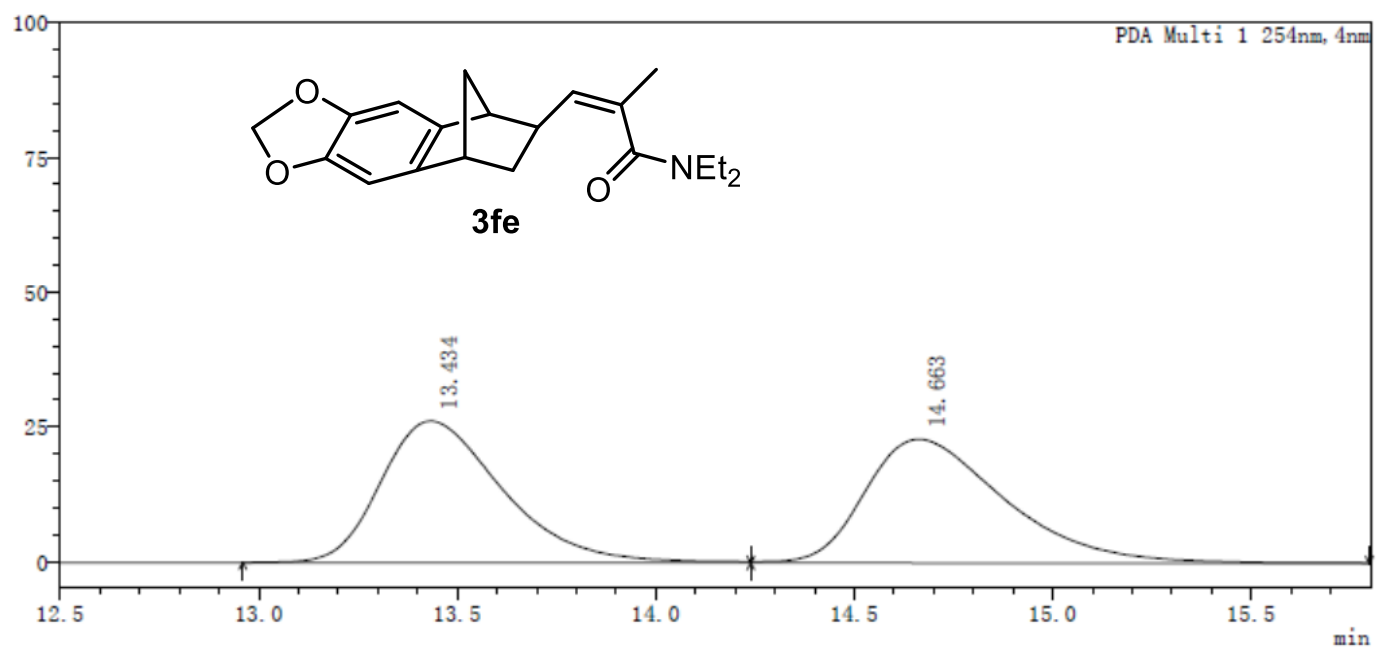

PDA Ch1 254nm

\begin{tabular}{|r|r|r|r|r|r|}
\hline 峰号 & 保留时间 & 峰宽 (高度 50\%) & 高度 & \multicolumn{1}{c|}{ 面积 } & \multicolumn{1}{c|}{ 面积\% } \\
\hline 1 & 13.434 & 0.325 & 26247 & 562321 & 49.987 \\
\hline 2 & 14.663 & 0.371 & 22956 & 562619 & 50.013 \\
\hline
\end{tabular}

Figure S105. Chiral HPLC chromatogram for racemic 3fe.

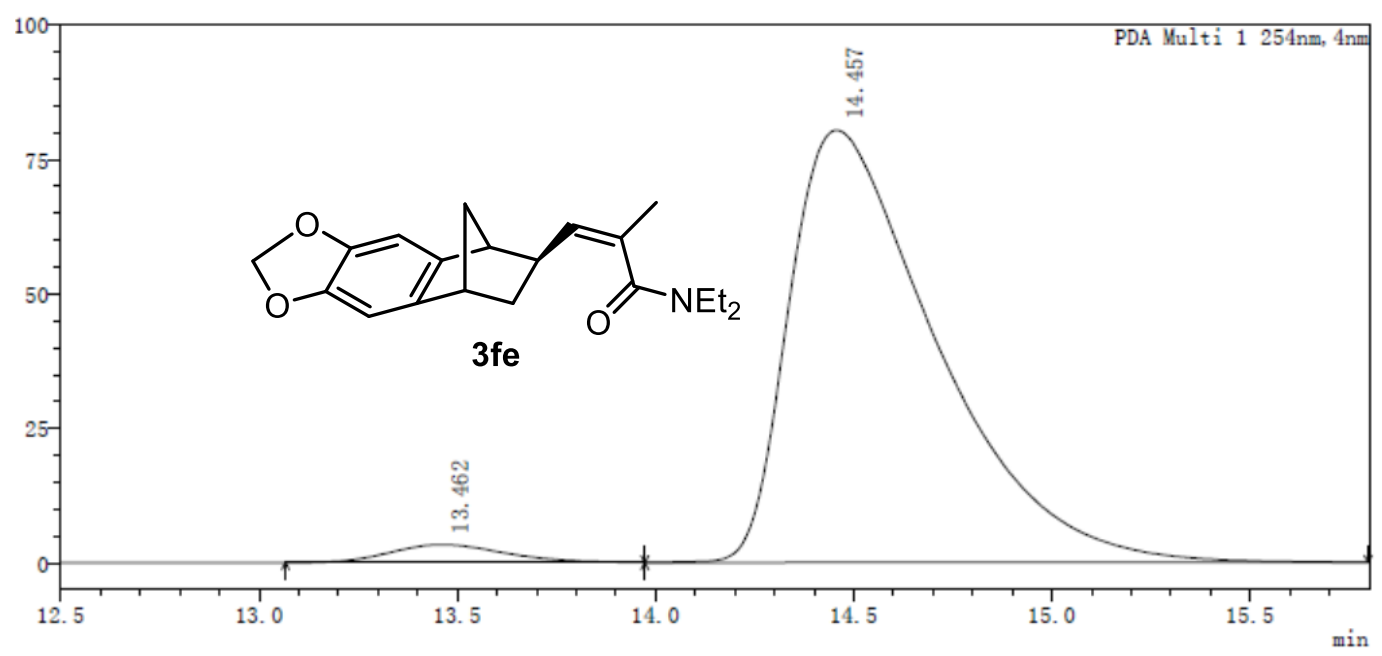

PDA Ch1 254nm

\begin{tabular}{|r|r|r|r|r|r|}
\hline 峰号 & 保留时间 & 峰宽 (高度 50\%) & 高度 & \multicolumn{1}{c|}{ 面积 } & \multicolumn{1}{c|}{ 面积\% } \\
\hline 1 & 13.462 & 0.314 & 3337 & 67809 & 3.151 \\
\hline 2 & 14.457 & 0.391 & 80397 & 2083955 & 96.849 \\
\hline
\end{tabular}

Figure S106. Chiral HPLC chromatogram for enantioenriched 3fe. 


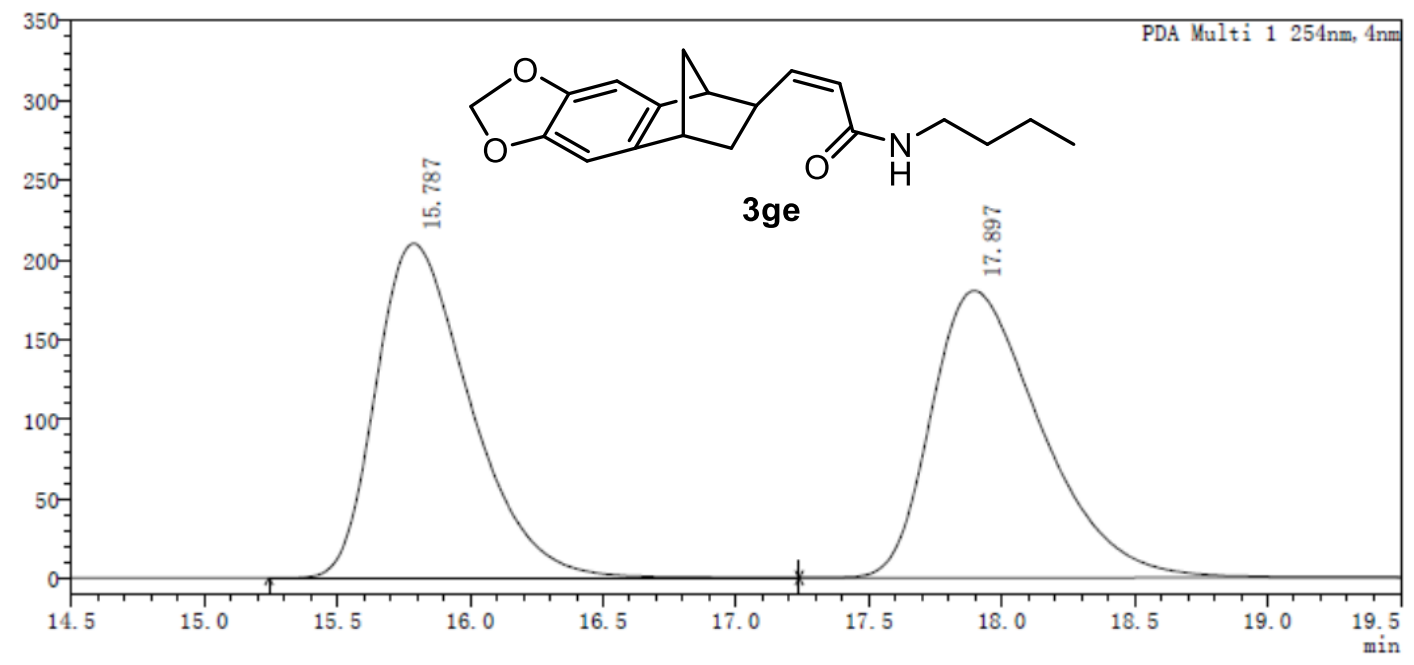

PDA Ch1 254nm

\begin{tabular}{|r|r|r|r|r|r|}
\hline 峰号 & 保留时间 & 峰宽 (高度 50\%) & 高度 & 面积 & \multicolumn{1}{c|}{ 面积\% } \\
\hline 1 & 15.787 & 0.379 & 209623 & 5181957 & 49.982 \\
\hline 2 & 17.897 & 0.441 & 179838 & 5185638 & 50.018 \\
\hline
\end{tabular}

Figure S107. Chiral HPLC chromatogram for racemic 3ge.

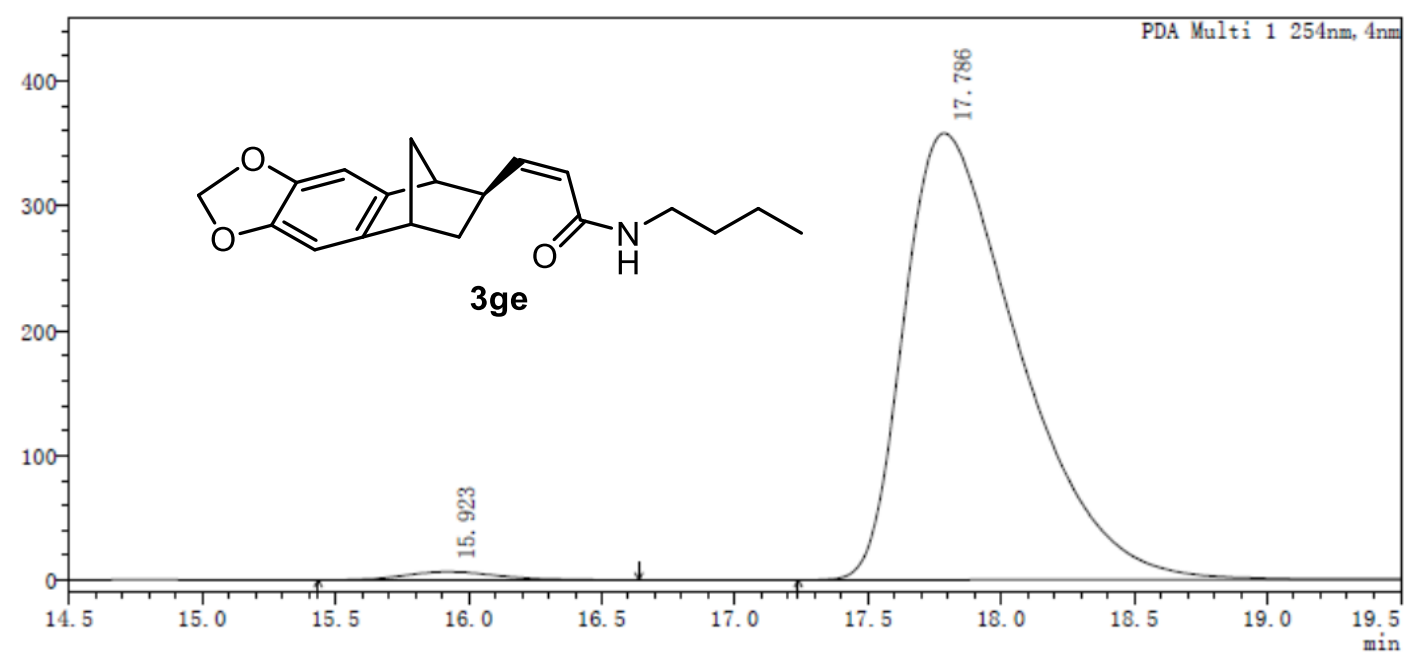

PDA Ch1 254nm

\begin{tabular}{|r|r|r|r|r|r|}
\hline 峰号 & 保留时间 & 峰宽 (高度 50\%) & 高度 & \multicolumn{1}{c|}{ 面积 } & \multicolumn{1}{c|}{ 面积\% } \\
\hline 1 & 15.923 & 0.362 & 6563 & 153146 & 1.404 \\
\hline 2 & 17.786 & 0.459 & 357671 & 10755318 & 98.596 \\
\hline
\end{tabular}

Figure S108. Chiral HPLC chromatogram for enantioenriched 3ge. 


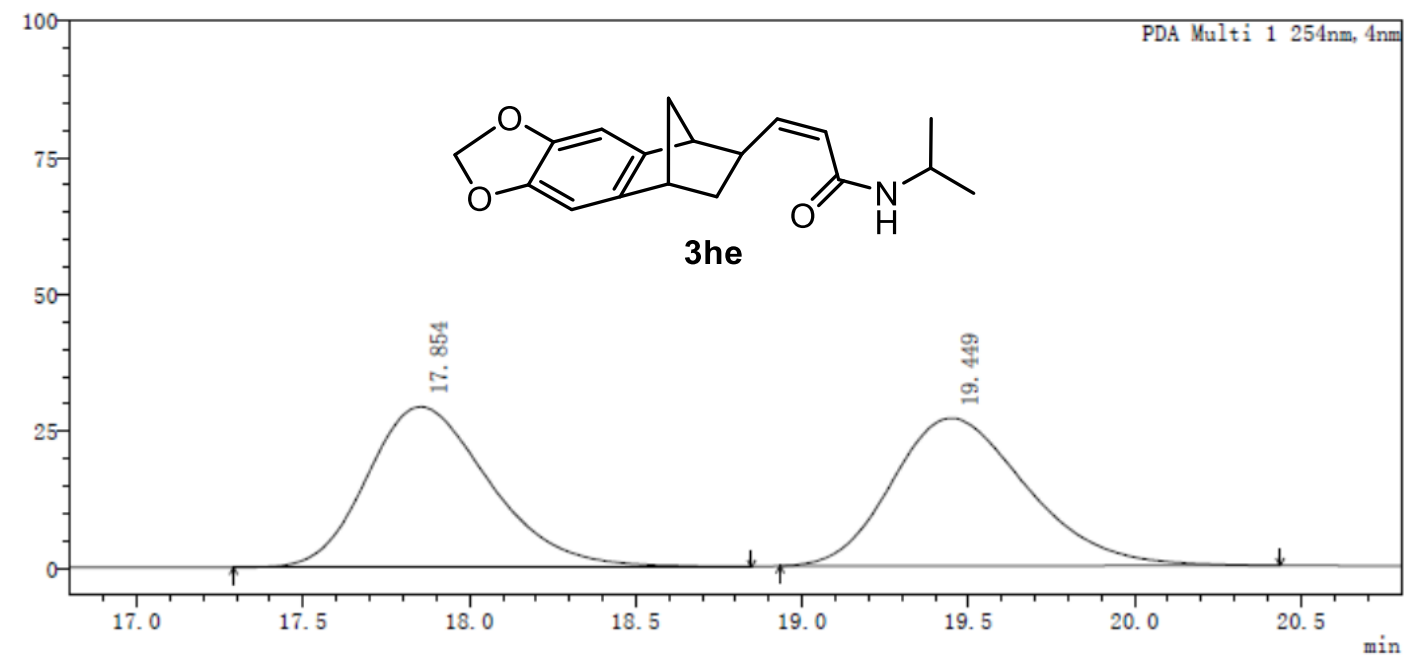

\begin{tabular}{|c|c|c|c|c|c|}
\hline \multicolumn{6}{|c|}{$\mathrm{DACh}$} \\
\hline 峰号 & 保留时间 & 峰宽 (高度 $50 \%$ ) & 高度 & 面积 & 面积\% \\
\hline 1 & 17.854 & 0.394 & 29375 & 759903 & 49. 904 \\
\hline 2 & 19. 449 & 0.436 & 26973 & 762841 & 50.096 \\
\hline
\end{tabular}

Figure S109. Chiral HPLC chromatogram for racemic 3 he.

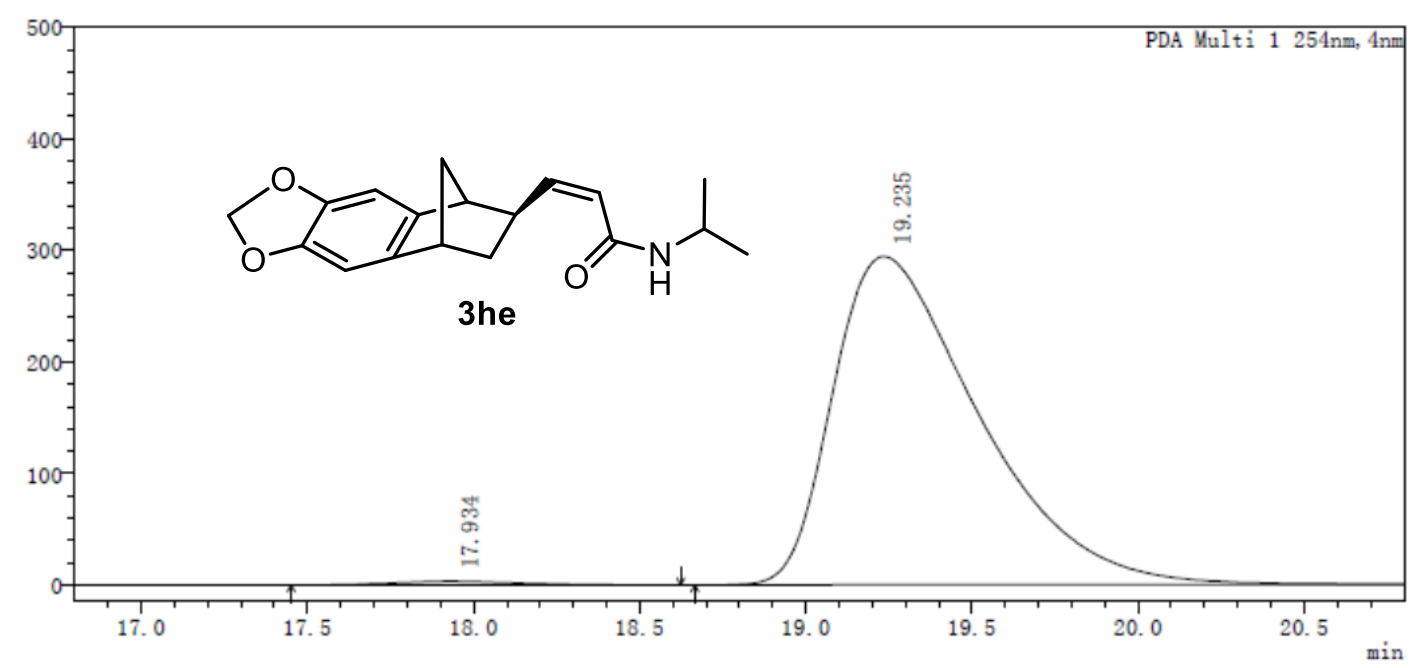

PDA Ch1 254nm

\begin{tabular}{|r|r|r|r|r|r|}
\hline 峰号 & 保留时间 & 峰宽 (高度 50\%) & \multicolumn{1}{|c|}{ 高度 } & \multicolumn{1}{c|}{ 面积 } & \multicolumn{1}{c|}{ 面积\% } \\
\hline 1 & 17.934 & 0.382 & 3098 & 76234 & 0.837 \\
\hline 2 & 19.235 & 0.467 & 294352 & 9029773 & 99.163 \\
\hline
\end{tabular}

Figure S110. Chiral HPLC chromatogram for enantioenriched 3he. 


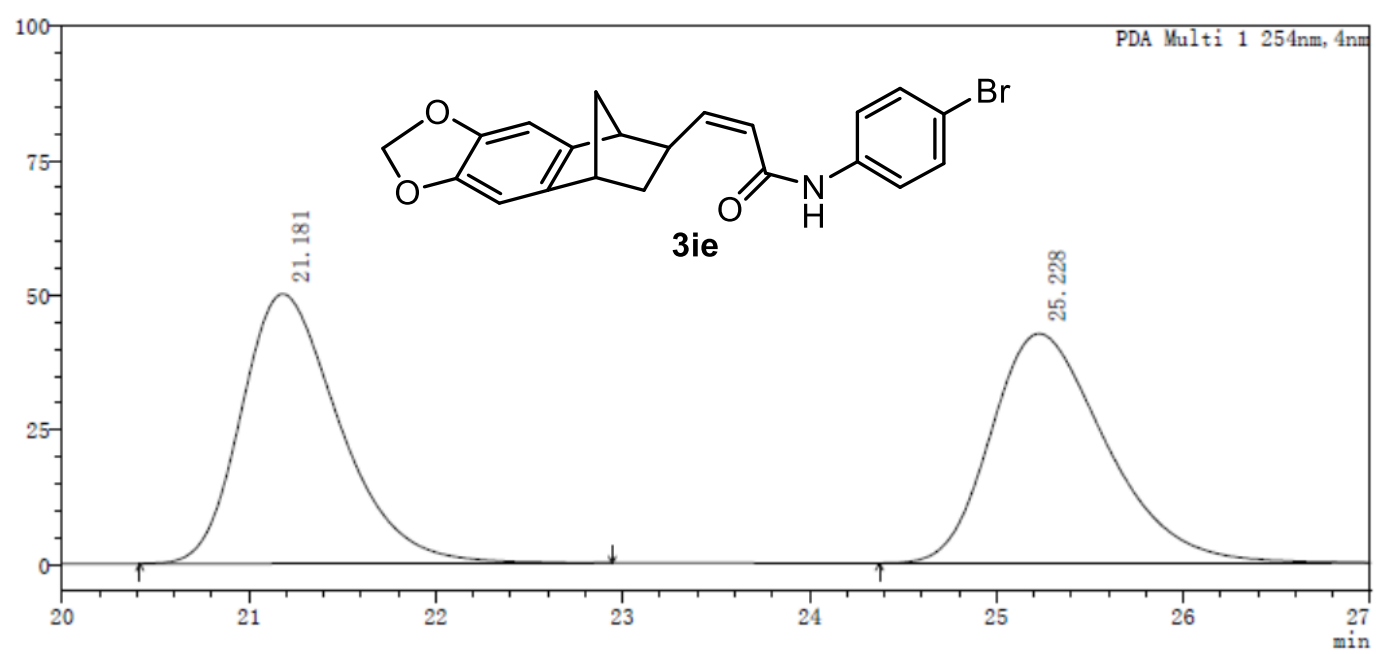

PDA Ch1 254nm

\begin{tabular}{|r|r|r|r|l|l|}
\hline 峰号 & 保留时间 & 峰宽 (高度 50\%) & 高度 & 面积 & \multicolumn{1}{c|}{ 面积\% } \\
\hline 1 & 21.181 & 0.557 & 50056 & 1833201 & 49.978 \\
\hline 2 & 25.228 & 0.657 & 42639 & 1834830 & 50.022 \\
\hline
\end{tabular}

Figure S111. Chiral HPLC chromatogram for racemic 3ie.

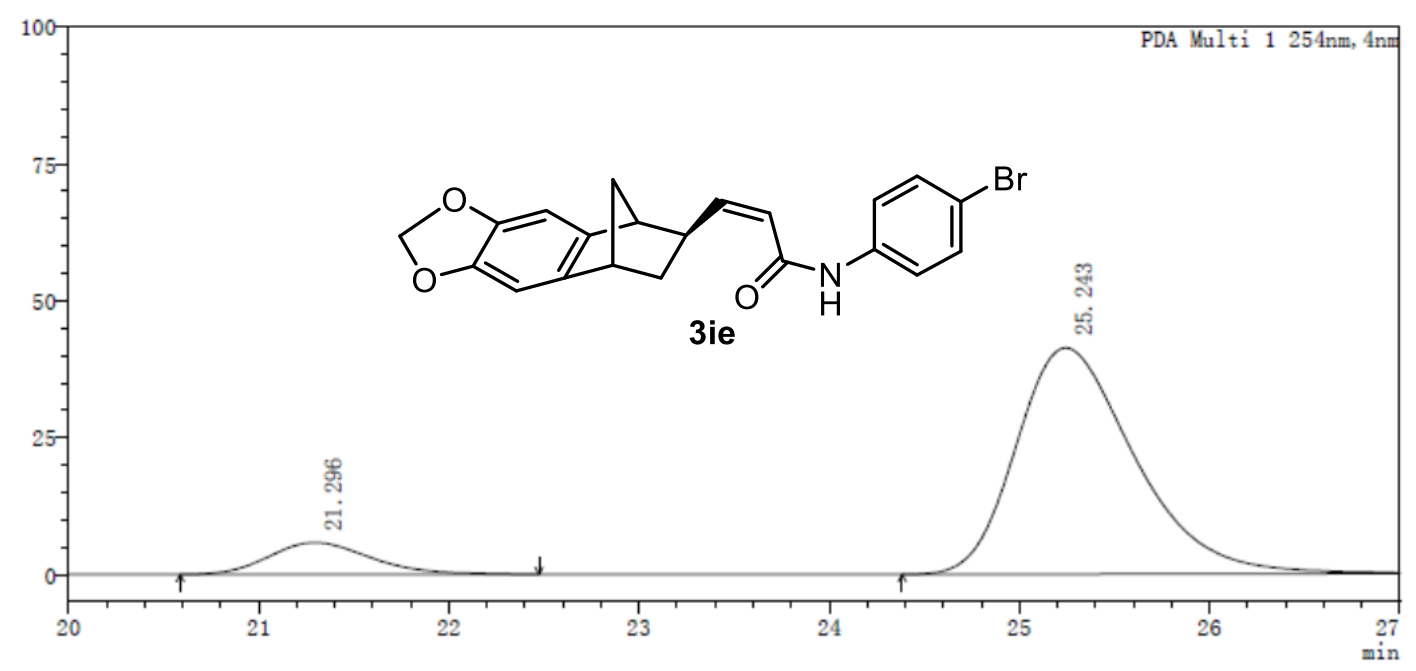

PDA Ch1 254nm

\begin{tabular}{|r|r|r|r|r|r|}
\hline 峰号 & 保留时间 & 峰宽 (高度 50\%) & 高度 & \multicolumn{1}{c|}{ 面积 } & \multicolumn{1}{c|}{ 面积\% } \\
\hline 1 & 21.296 & 0.571 & 5799 & 215831 & 10.699 \\
\hline 2 & 25.243 & 0.660 & 41344 & 1801379 & 89.301 \\
\hline
\end{tabular}

Figure S112. Chiral HPLC chromatogram for enantioenriched 3ie. 


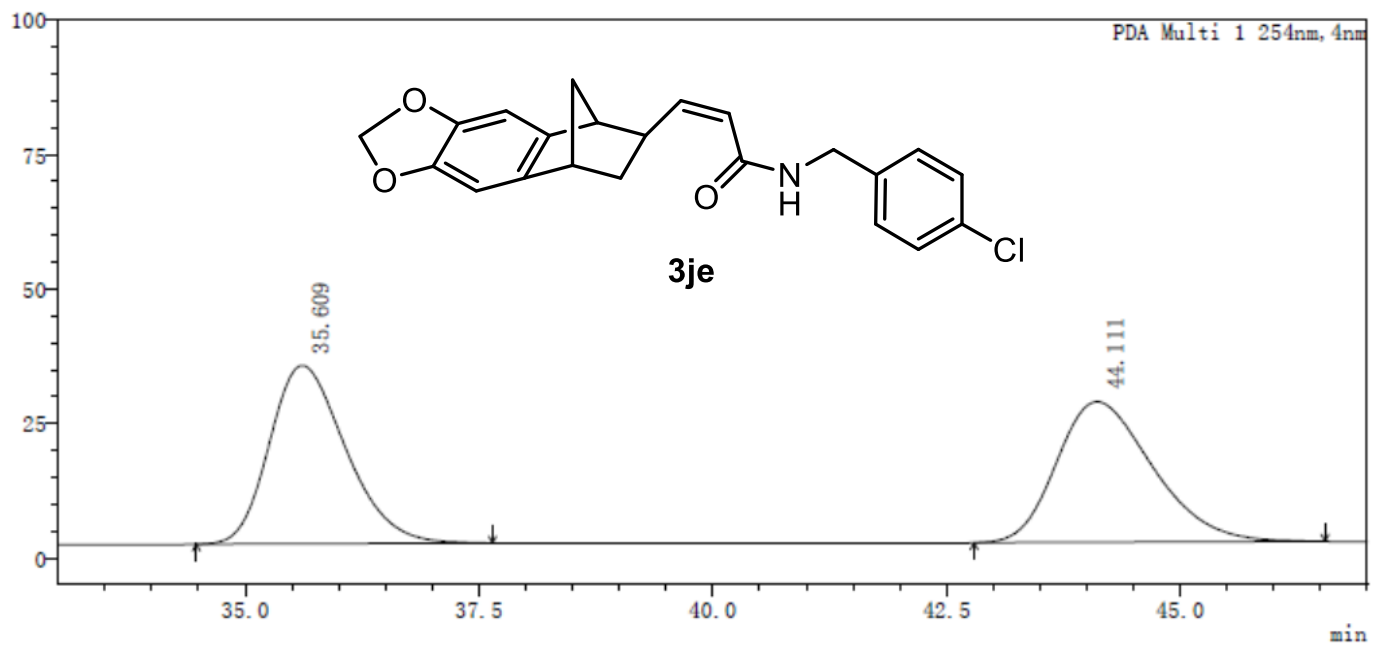

PDA Ch1 254nm

\begin{tabular}{|r|r|r|r|r|r|}
\hline 峰号 & 保留时间 & 峰宽 (高度 50\%) & 高度 & 面积 & 面积\% \\
\hline 1 & 35.609 & 0.876 & 33152 & 1880206 & 50.103 \\
\hline 2 & 44.111 & 1.109 & 26125 & 1872440 & 49.897 \\
\hline
\end{tabular}

Figure S113. Chiral HPLC chromatogram for racemic 3je.

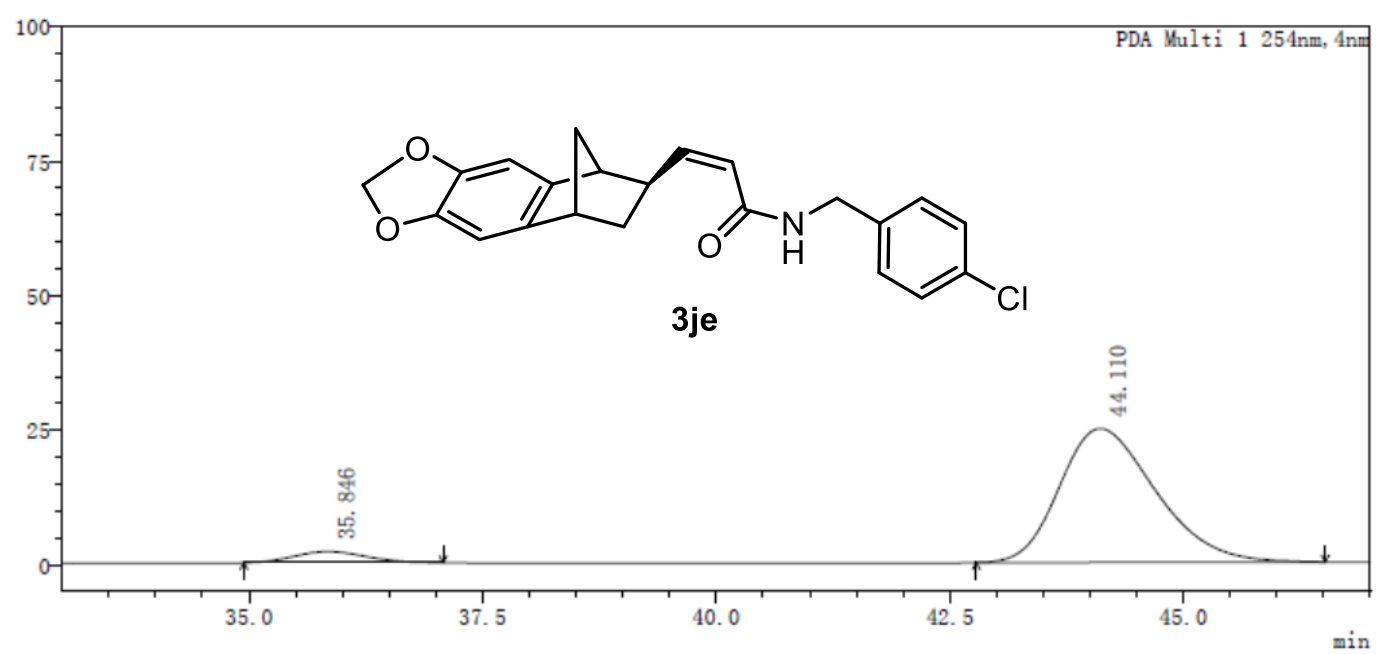

PDA Ch1 254nm

\begin{tabular}{|r|r|r|r|r|r|} 
PDA Ch1 $254 \mathrm{~nm}$ \\
\hline 1 & 35.846 & 0.852 & 2070 & 111655 & 5.909 \\
\hline 2 & 44.110 & 1.106 & 24853 & 1777949 & 94.091 \\
\hline
\end{tabular}

Figure S114. Chiral HPLC chromatogram for enantioenriched 3je. 


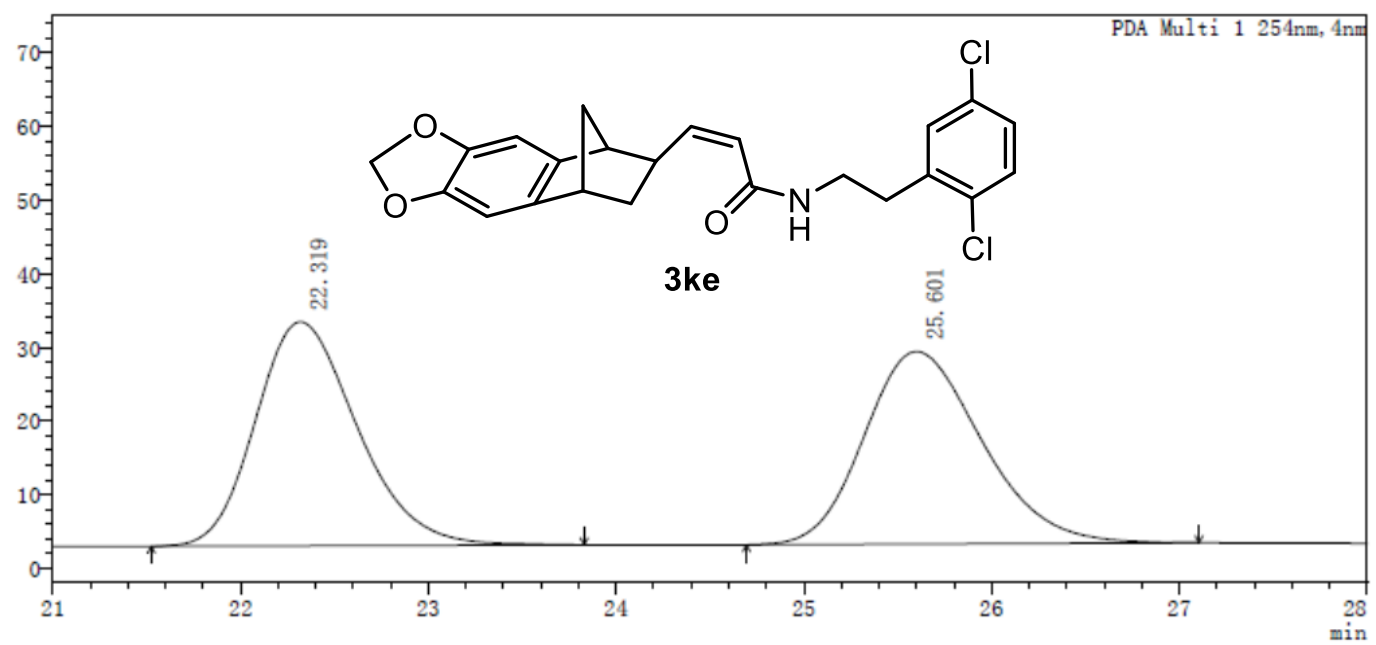

PDA Ch1 254nm

\begin{tabular}{|r|r|r|r|r|r|}
\hline 峰号 & 保留时间 & 峰宽 (高度 50\%) & 高度 & 面积 & \multicolumn{1}{c|}{ 面积\% } \\
\hline 1 & 22.319 & 0.579 & 30390 & 1143164 & 50.116 \\
\hline 2 & 25.601 & 0.673 & 26122 & 1137854 & 49.884 \\
\hline
\end{tabular}

Figure S115. Chiral HPLC chromatogram for racemic 3ke.

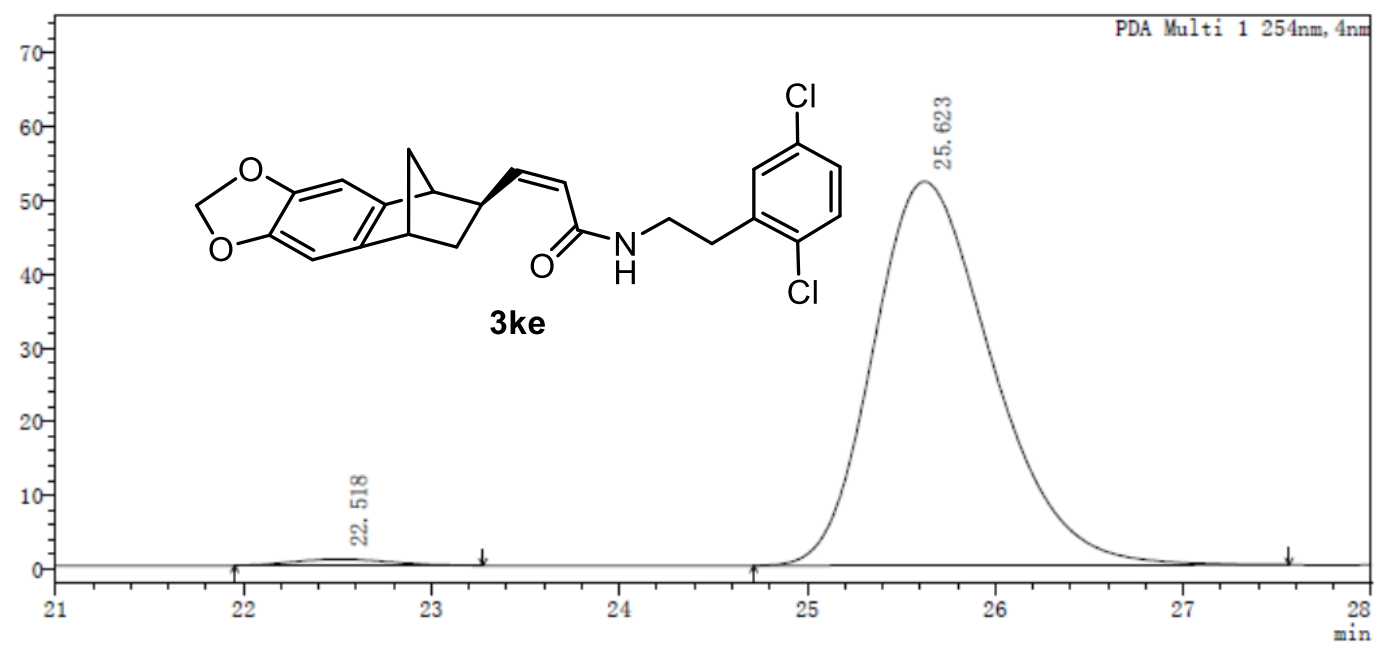

PDA Ch1 $254 \mathrm{~nm}$

\begin{tabular}{|r|r|r|r|r|r|}
\hline 峰号 & 保留时间 & 峰宽(高度 $50 \%$ ) & \multicolumn{1}{c|}{ 高度 } & \multicolumn{1}{c|}{ 面积 } & \multicolumn{1}{c|}{ 面积\% } \\
\hline 1 & 22.518 & 0.570 & 861 & 30794 & 1.324 \\
\hline 2 & 25.623 & 0.678 & 52045 & 2294453 & 98.676 \\
\hline
\end{tabular}

Figure S116. Chiral HPLC chromatogram for enantioenriched 3ke. 


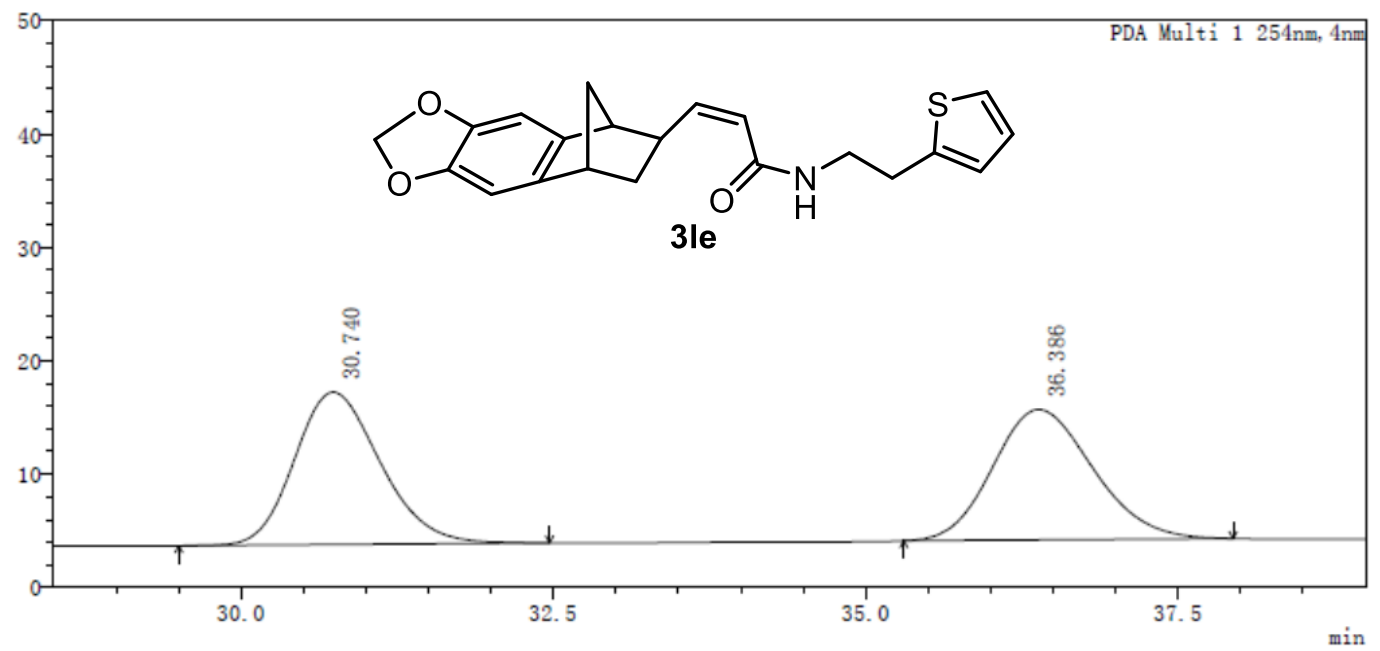

PDA Ch1 254nm

\begin{tabular}{|r|r|r|r|r|r|}
\hline 峰号 & 保留时间 & 峰宽 (高度 50\%) & 高度 & 面积 & \multicolumn{1}{c|}{ 面积\% } \\
\hline 1 & 30.740 & 0.737 & 13427 & 641237 & 50.001 \\
\hline 2 & 36.386 & 0.869 & 11490 & 641205 & 49.999 \\
\hline
\end{tabular}

Figure S117. Chiral HPLC chromatogram for racemic 3le.

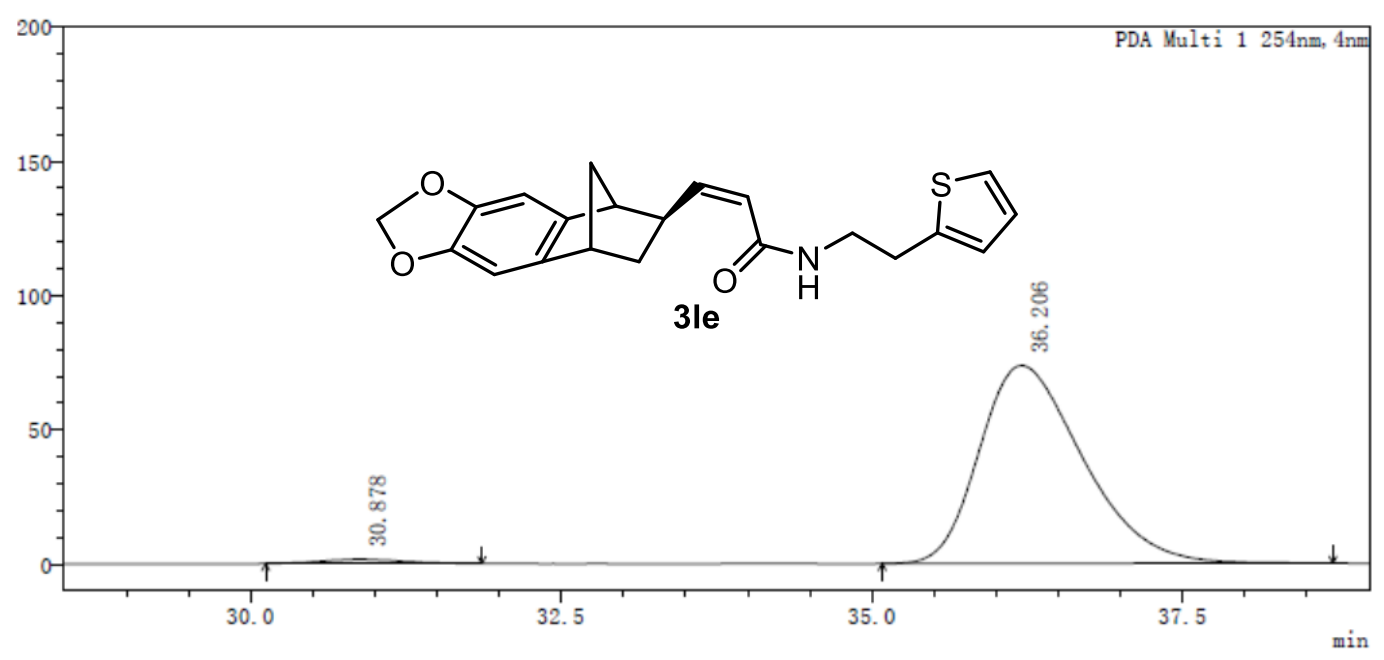

PDA Ch1 254nm

\begin{tabular}{|r|r|r|r|r|r|}
\hline 峰号 & 保留时间 & 峰宽 (高度 50\%) & 高度 & \multicolumn{1}{c|}{ 面积 } & 面积\% \\
\hline 1 & 30.878 & 0.719 & 1587 & 71915 & 1.654 \\
\hline 2 & 36.206 & 0.895 & 73708 & 4276081 & 98.346 \\
\hline
\end{tabular}

Figure S118. Chiral HPLC chromatogram for enantioenriched 3le. 


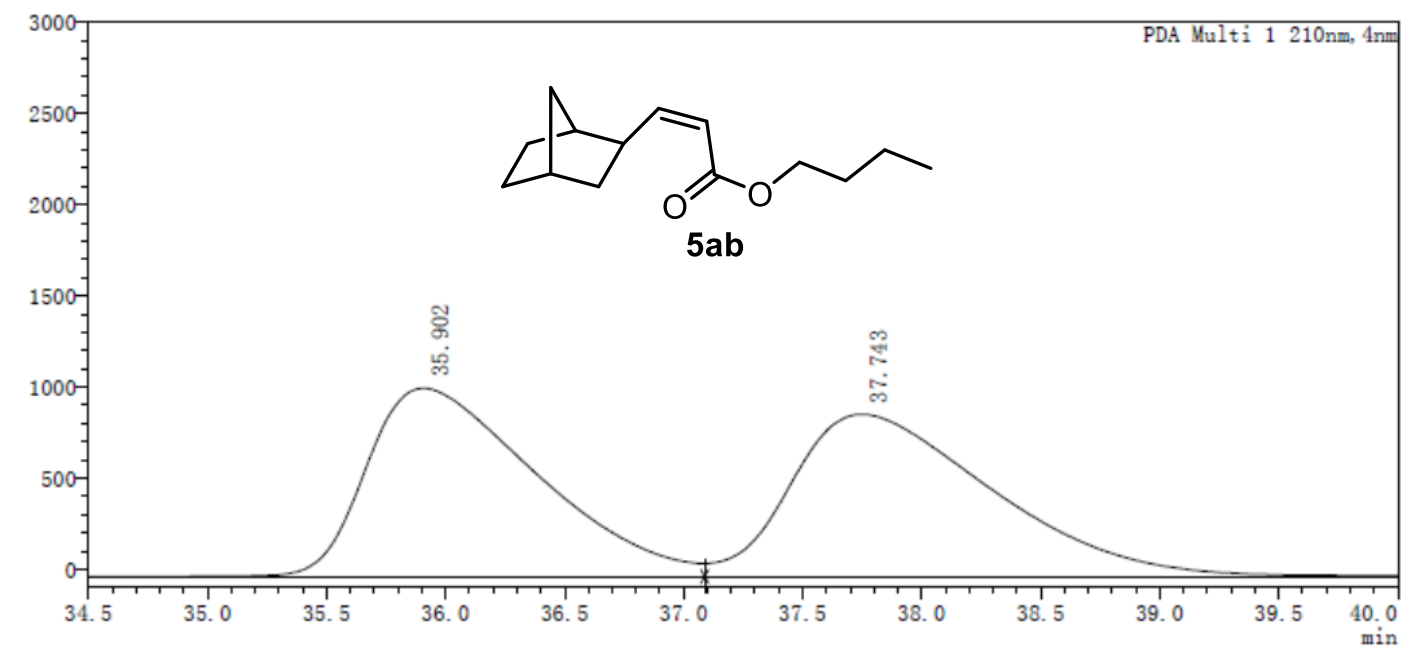

PDA Ch1 210nm

\begin{tabular}{|r|r|r|r|r|r|}
\hline 峰号 & 保留时间 & 峰宽 (高度 50\%) & 高度 & 面积 & 面积\% \\
\hline 1 & 35.902 & 0.779 & 1031359 & 51138173 & 49.514 \\
\hline 2 & 37.743 & 0.907 & 888507 & 52141421 & 50.486 \\
\hline
\end{tabular}

Figure S119. Chiral HPLC chromatogram for racemic 5ab.

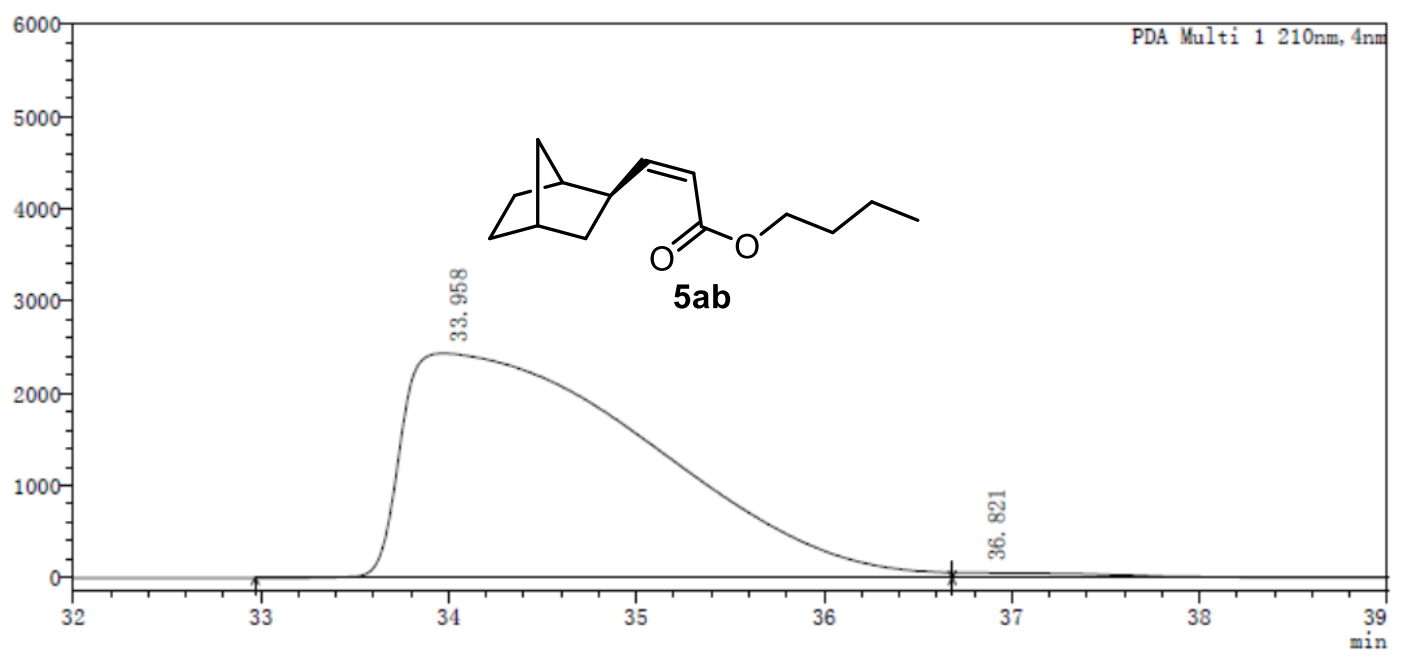

PDA Ch1 210nm

\begin{tabular}{|r|r|r|r|r|r|}
\hline 峰号 & 保留时间 & 峰宽 (高度 50\%) & 高度 & 面积 & 面积\% \\
\hline 1 & 33.958 & 1.505 & 2432329 & 224104111 & 98.629 \\
\hline 2 & 36.821 & -- & 51848 & 3114654 & 1.371 \\
\hline
\end{tabular}

Figure S120. Chiral HPLC chromatogram for enantioenriched 5ab. 